\title{
Site-Selective N-Dealkylation of 1,2,3-Triazolium Salts: a Metal-free Route to 1,5-Substituted 1,2,3-Triazoles and Related Bistriazoles.
}

\author{
Zaira Monasterio, Aitziber Irastorza, José I. Miranda and Jesús M. Aizpurua* \\ Department of Organic Chemistry I, University of the Basque Country UPV/EHU \\ Joxe Mari Korta R\&D Center, Avda Tolosa 72, 20018 Donostia-San Sebastián (Spain)
}

E-mail:jesusmaria.aizpurua@ehu.es

\section{SUPPORTING INFORMATION}

1. Preparation details and physical and spectroscopic data of compounds $1 \mathrm{~b}, \mathbf{1 f}-\mathrm{g}, \mathbf{1 j}$ k, 2a-m, 6-9, 11-13, 15, 17-18.

page

1.1. General.

1.2. Synthesis of 2-[2-(2,4,6-triiodophenoxy)ethoxy]ethanol.

1.3. Synthesis of alkyl trifluromethanesulfonates.

S3

1.4. Synthesis of 1,4-disubstituted-1H-1,2,3-triazoles.

S4

1.5. Synthesis of $N$-alkyl-1H-1,2,3-triazolium trifluromethanesulfonates.

S5

1.6. Synthesis of 1,5-disubstituted-1H-1,2,3-triazoles.

1.7. Synthesis of 1,4,5-trisubstituted 1,2,3-triazoles.

$\mathrm{S} 13$

1.8. Synthesis of bistriazoles and tetrahydrohexaazaindacenium salts.

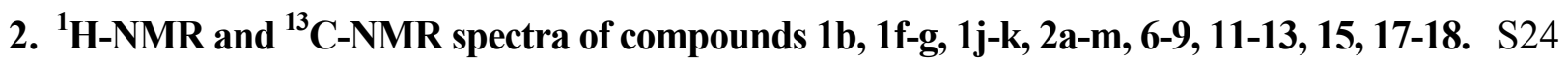

3. N-Dealkylation of 1-benzyl-3-methyl-4-phenyl-1,2,3-triazolium salt 2n. $\quad$ S67

4. N-Dealkylation of 1-pivayloxymethyl-1,2,3-triazolium salts. $\quad$ S68

5. Computational calculations. $\quad \mathrm{S} 72$

5.1. Computational methods. $\quad \mathrm{S} 72$

5.2. Electronic energies and cartesian coordinates for structures $\mathbf{D}$ and $\mathbf{E}$ NBO charges. S73

5.3. Comparison of the free energy profiles of the hydroxide-promoted $\mathrm{N}$ dealkylation of a 1,2,3-triazole and 1,2,3-triazolium salt comprising pivaloyloxymethyl group. Electronic energies and cartesian coordinates.

5.4. Comparison of the free energy profiles of the intramolecular and intermolecular thermal cycloaddition reaction of 4-ethynyl-1-methyl-1,2,3-triazolium cations. Electronic energies and cartesian coordinates. 


\section{Preparation details and physical and spectroscopic data of compounds 1b, 1f-g, 1j-k, 2a-m, 6-9, 11-13, 15, 17-18.}

\subsection{General}

All reagents and solvents were obtained from commercial sources (Aldrich, Acros, Merck, Sigma and Fluka) and were used without further purification unless stated otherwise. Tetrahydrofuran (THF) and diethyl ether $\left(\mathrm{Et}_{2} \mathrm{O}\right)$ were dried through PS-MD-2columns. Extra pure dichloromethane $\left(\mathrm{CH}_{2} \mathrm{Cl}_{2}\right)$, acetonitrile $(\mathrm{MeCN})$, hexane $(\mathrm{Hex})$ and ethyl acetate (EtOAc) were bought from Sharlau. Moisture sensitive reactions were carried out with magnetic stirring under an atmosphere of nitrogen in oven- or flame-dried glassware.

Purification of reaction products was carried out by flash chromatography using silica gel 60 (230-400 mesh). Analytical thin layer chromatography was performed on $0.25 \mathrm{~mm}$ silica gel 60-F plates. Visualization was accomplished with UV light and phosphomolybdic acid-ammonium cerium (IV) nitric-sulfuric acid-water reagent, followed by heating.

${ }^{1} \mathrm{H}$ NMR spectra were recorded on Bruker spectrometers at 500 and $400 \mathrm{MHz} \cdot{ }^{13} \mathrm{C}$ NMR spectra were recorded at 125 and $101 \mathrm{MHz}$. The chemical shifts are reported as $\delta$ values $(\mathrm{ppm})$ relative to residual deuterated solvent as internal standards: for $\mathrm{CDCl}_{3} \delta \mathrm{H}(7.26$ $\mathrm{ppm})$ and $\delta \mathrm{C}(77.16 \mathrm{ppm})$, respectively; for $\mathrm{MeOH}-\mathrm{d}_{4} \delta \mathrm{H}(3.31 \mathrm{ppm})$ and $\delta \mathrm{C}(49.0 \mathrm{ppm})$, respectively; for $\mathrm{MeCN}_{-} \mathrm{d}_{3} \delta \mathrm{H}(1.94 \mathrm{ppm})$ and $\delta \mathrm{C}(118.26 \mathrm{ppm}, 1.32 \mathrm{ppm})$, respectively; for DMSO- $\mathrm{d}_{6} \delta \mathrm{H}(2.50 \mathrm{ppm})$ and $\delta \mathrm{C}(39.52 \mathrm{ppm})$, respectively.

Mass spectra were acquired on a time of flight (TOF) mass spectrometer (SYNAPT G2 HDMS from Waters, Milford, MA, USA) equipped with an electrospray source in positive mode (ESI+). Melting points were measured with a Büchi SMP-20 melting point apparatus and are uncorrected. Infrared spectra were recorded on a Bruker Alpha $P$ spectrometer. Optical rotations were measured on a Jasco P-200 polarimeter using a sodium lamp (589 nm, D line) at $25 \pm 0.2^{\circ} \mathrm{C}$.

The following compounds were prepared according to previously described procedures: 2(2,4,6-triiodophenoxy)ethanol), ${ }^{1}$ 4-tert-butylbenzyl azide, ${ }^{2}$ benzyl azide, ${ }^{3}$ (3R)-(2-azidoacetylamino)-3-phenyl-propionic acid methyl ester, ${ }^{4}$ azidomethyl pivalate $8,{ }^{5} \mathrm{~N}, N$ '-di-Boc$N^{\prime \prime}$ '(but-3-ynyl)-guanidine, ${ }^{4} \quad N, N^{\prime}$-di-Boc- $N$ ''-(pent-4-ynyl)-guanidine, ${ }^{4} \quad$ 2-azidoethyl

1 Radek, O.; Nemecek, O. Czech Patent 1966, CS 118450.

2 Barr, L.; Lincoln, S. F.; Easton, C. J. Supramol. Chem. 2005, 17, 547-555.

3 Alvarez, S. G.; Alvarez, M. T. Synthesis 1997, 413-414.

4 Trabocchi, A.; Menchi, G.; Cini, N.; Bianchini, F.; Raspanti, S.; Bottoncetti, A.; Pupi, A.; Calorini, L.; Guarna, A. J. Med. Chem. 2010, 53, 7119-7128.

5 Rajamalleswaramma, J.; Jürgen, R.; Jens, C. Synlett. 2007, 2847. 
trifluoromethanesulfonate, ${ }^{6}$ 3-azidopropyl-1- trifluoromethanesulfonate $\mathbf{1 4},{ }^{7}$ butyl trifluoromethanesulfonate, ${ }^{8}$ hex-5-yn-1-yl triflate ${ }^{9}$, 3-bromopropyl trifluoromethanesulfonate, ${ }^{10}$ phenethyl trifluoromethanesulfonate, $,{ }^{11} \mathbf{1 a},{ }^{12} \mathbf{1 c},{ }^{13} \mathbf{1 d},{ }^{14} \mathbf{1 e},{ }^{15} \mathbf{1 h},{ }^{16} \mathbf{1 i},{ }^{17} 4$-[1-(4fluorophenyl)-1H-1,2,3-triazol-4-yl]-3-methyl-1-phenyl-1 $H$-1,2,3-triazolium tetrafluoroborate, ${ }^{18} \quad 3$-methyl-1-phenyl-4-[1-(2,4,6-trimethylphenyl)-1 $H$-1,2,3-triazol-4-yl]-1 $H$-1,2,3triazolium tetrafluoroborate, ${ }^{18} \quad 4$-[1-(2,6-diisopropylphenyl)-1H-1,2,3-triazol-4-yl]-3methyl-1-phenyl-1H-1,2,3-triazolium tetrafluoroborate. ${ }^{18}$

\subsection{Synthesis of 2-[2-(2,4,6-triiodophenoxy)ethoxy]ethanol}

\section{2-[2-(2,4,6-Triiodophenoxy)ethoxy]ethanol}

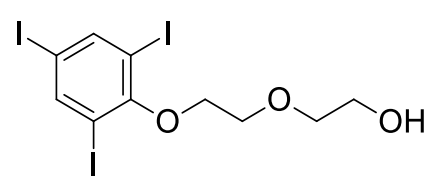

To a solution of 2,4,6-triiodophenol $(0.42 \mathrm{mmol}, 200 \mathrm{mg})$ in EtOH $(2.2 \mathrm{~mL})$ was added $\mathrm{NaOH}(0.38 \mathrm{mmol}, 15 \mathrm{mg})$ and the reaction mixture was stirred for $30 \mathrm{~min}$ at ambient temperature. Then, the solvent was evaporated under reduced pressure. Then, NaI $(0.38 \mathrm{mmol}, 57 \mathrm{mg})$ and 2-(2-chloroethoxy)ethanol $(0.38 \mathrm{mmol}, 40 \mu \mathrm{L})$ in $\mathrm{EtOH}(2.2 \mathrm{~mL})$ were added and the reaction mixture was stirred at $150{ }^{\circ} \mathrm{C}$ for 6 hours in an ACE pressure tube. Then, the solvent was evaporated under reduced pressure. A solution of $\mathrm{NaOH} 6 \mathrm{M}(6 \mathrm{~mL})$ was added and the product was extracted with $\mathrm{CH}_{2} \mathrm{Cl}_{2}(7 \mathrm{~mL}$ x 3$)$.

6 Takaya, T.; Tozuka, Z.; Yasuda, N.; Kawabata, K. 1985, BE 900230 A1 BE 19850128.

7 Kramer, J. R.; Deming, T. J. Biomacromolecules 2012, 13, 1719-1723.

8 Ross, S. A.; Pitíe, M.; Meunier, B. J. Chem. Soc., Perking Trans. 1 2000, 571-574.

9 Severa, L.; Adriaenssens, L.; Vávra, J.; Saman, D.; Císarová, I.; Fiedler, P.; Teplý, F. Tetrahedron 2010, 13, 1719.

10 Hugelshofer, C. L.; Mellem, K. T.; Myers, A. G. Org. Lett. 2013, 15, 3135.

11 Dang, H.; Mailig, M.; Lalic, G. Angew. Chem. Int. Ed. 2014, 53, 6473.

12 Jlalia, I.; Meganem, F.; Herscovici, J.; Girard, C. Molecules 2009, 14, 528-539.

13 Wu, Y.-M.; Deng, J.; Li, Y.; Chen, Q.-Y. Synthesis 2005, 1314-1318.

14 Aizpurua, J. M.; Azcune, I.; Fratila, R. M.; Balentova, E.; Sagartzazu-Aizpurua, M.; Miranda, J, I. Org. Lett. 2010, 12, 1584-1587.

15 Buckley, B. R.; Dann, S. E.; Harris, D. P.; Heaney, H.; Stubbs, E. C. Chem. Commun. 2010, 46, 2274-2276.

16 Aizpurua, J. M.; Sagartzazu-Aizpurua, M.; Monasterio, Z.; Azcune, I.; Mendicute, C.; Miranda, J. I.; García-Lecina, E.; Altube, A.; Fratila, R. M. Org. Lett. 2012, 14, 1866-1868.

17 Thompson, R. L.; Damodaran, K.; Luebke, D.; Nulwala, H. Synlett 2013, 1093.

18 Monasterio, Z.; Sagartzazu-Aizpurua, M.; Miranda, J. I.; Reyes, Y.; Aizpurua, J. M. Org. Lett. 2016, 18,788 . 
The organic layer was dried over $\mathrm{MgSO}_{4}$ and evaporated to dryness. The product was purified by column chromatography (silica gel, Hex/EtOAc 1:1). Yield: $103 \mathrm{mg}$ (48 \%). White solid $\left(\mathrm{mp}=76-78{ }^{\circ} \mathrm{C}\right) .{ }^{1} \mathrm{H}$ NMR $\left(400 \mathrm{MHz}, \mathrm{CDCl}_{3}\right): \delta 8.07$ (s, 2H), $4.16(\mathrm{t}, J=4.6$ $\mathrm{Hz}, 2 \mathrm{H}), 3.99$ (t, $J=4.6 \mathrm{~Hz}, 2 \mathrm{H}), 3.86-3.78(\mathrm{~m}, 2 \mathrm{H}), 3.78-3.70(\mathrm{~m}, 2 \mathrm{H}) .{ }^{13} \mathrm{C}$ NMR $(101$ $\left.\mathrm{MHz}, \mathrm{CDCl}_{3}\right): \delta 158.0,147.6,92.2,89.7,72.8,72.5,70.2,62.1 . \mathrm{IR}\left(\mathrm{cm}^{-1}\right): 1409,1357$, 1236, 1113, 1068, 1042, 1030, 1007. HRMS (ESI+): $\mathrm{m} / z$ [M+Na] ${ }^{+}$calcd for $\mathrm{C}_{10} \mathrm{H}_{11} \mathrm{O}_{3} \mathrm{I}_{3} \mathrm{Na}$ : 582.7734; found: 582.7756 .

\subsection{Synthesis of alkyl trifluoromethanesulfonates}

General Procedure: Trifluoromethanesulfonic anhydride $\mathrm{Tf}_{2} \mathrm{O}(1.20 \mathrm{mmol})$ was added dropwise over a period of $5 \mathrm{~min}$ to a vigorously stirred solution of the corresponding alcohol $(1.00 \mathrm{mmol})$ and dehydrated $\mathrm{KHCO}_{3}(1.40 \mathrm{mmol})$ in anhydrous $\mathrm{CH}_{2} \mathrm{Cl}_{2}(8.6 \mathrm{~mL})$ cooled to $0{ }^{\circ} \mathrm{C}$ and the reaction mixture was stirred for 5-18 hours at ambient temperature. The suspension was filtered through a $0.2 \mu \mathrm{m}$ PTFE filter and the resulting alkyl trifluoromethanesulfonate solution was used in the next alkylation reactions without further purification. Alternatively, the pure product could be isolated by evaporating the solvent at reduced pressure.

\section{2-(2,4,6-Triiodophenoxy)ethyl trifluoromethanesulfonate}

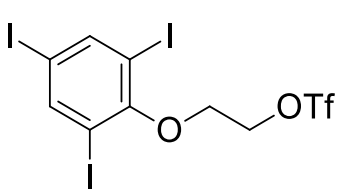

The general procedure was followed starting from 2-(2,4,6triiodophenoxy)ethanol $(0.58 \mathrm{mmol}, 300 \mathrm{mg}), \mathrm{KHCO}_{3}(0.81 \mathrm{mmol}$, $81 \mathrm{mg})$ and $\mathrm{Tf}_{2} \mathrm{O}(0.70 \mathrm{mmol}, 117 \mu \mathrm{L})$ in $\mathrm{CH}_{2} \mathrm{Cl}_{2}(5 \mathrm{~mL})$. The reaction mixture was stirred for 18 hours. Yield: $355 \mathrm{mg}$ (94\%).

White solid $\left(\mathrm{mp}=62-63{ }^{\circ} \mathrm{C}\right) .{ }^{1} \mathrm{H}$ NMR $\left(400 \mathrm{MHz}, \mathrm{CDCl}_{3}\right): \delta 8.10(\mathrm{~s}, 2 \mathrm{H}), 4.95(\mathrm{t}, J=4.2$ $\mathrm{Hz}, 2 \mathrm{H}), 4.32(\mathrm{t}, J=4.2 \mathrm{~Hz}, 2 \mathrm{H}) .{ }^{13} \mathrm{C} \mathrm{NMR}\left(101 \mathrm{MHz}, \mathrm{CDCl}_{3}\right): \delta 156.6,147.5,118.6(\mathrm{q}, J$ $=320.5,319.9 \mathrm{~Hz}), 91.5,90.3,74.5,69.2 \mathrm{IR}\left(\mathrm{cm}^{-1}\right): 1238,1200,1141,930,863,612$.

\section{2-[2-(2,4,6-Triiodophenoxy)ethoxy]ethyl trifluoromethanesulfonate}

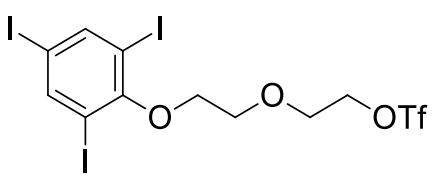

The general procedure was followed starting from 2-[2-(2,4,6triiodophenoxy)ethoxy]ethanol $(0.05 \mathrm{mmol}, 30 \mathrm{mg}), \mathrm{KHCO}_{3}$ $(0.08 \mathrm{mmol}, 8 \mathrm{mg})$ and $\mathrm{Tf}_{2} \mathrm{O}(0.07 \mathrm{mmol}, 11 \mu \mathrm{L})$ in $\mathrm{CH}_{2} \mathrm{Cl}_{2}(1$ $\mathrm{mL})$. The reaction mixture was stirred for 18 hours. Yield: quantitative. White solid $\left(\mathrm{mp}=65-67{ }^{\circ} \mathrm{C}\right) .{ }^{1} \mathrm{H}$ NMR $\left(400 \mathrm{MHz}, \mathrm{CDCl}_{3}\right): \delta 8.09(\mathrm{~s}, 2 \mathrm{H})$, 
$4.71(\mathrm{t}, J=4.1 \mathrm{~Hz}, 2 \mathrm{H}), 4.18(\mathrm{t}, J=4.6 \mathrm{~Hz}, 2 \mathrm{H}), 4.10-3.92(\mathrm{~m}, 4 \mathrm{H}) .{ }^{13} \mathrm{C} \mathrm{NMR}(101 \mathrm{MHz}$, $\left.\mathrm{CDCl}_{3}\right): \delta 157.7,147.4,91.8,89.5,75.5,72.4,70.4,68.7 . \mathrm{IR}\left(\mathrm{cm}^{-1}\right): 1243,1197,1138$, $1050,915,860,608$.

\subsection{Synthesis of 1,4-disubstituted-1H-1,2,3-triazoles}

General Procedure: To a solution of the corresponding azide $(1.00 \mathrm{mmol})$ and alkyne (1.10 mmol) in $\mathrm{THF} /{ }^{t} \mathrm{BuOH} / \mathrm{H}_{2} \mathrm{O} 1: 1: 1$ (5 mL), sodium ascorbate $(0.40 \mathrm{mmol})$ and $\mathrm{CuSO}_{4} \cdot 5 \mathrm{H}_{2} \mathrm{O}(0.20 \mathrm{mmol})$ were added. The reaction mixture was stirred at ambient temperature for 18 hours. The organic solvents were evaporated under reduced pressure and the aqueous residue was partitioned between EtOAc and a $20 \%$ aqueous $\mathrm{NH}_{3}$ solution. The organic layer was separated, dried over $\mathrm{MgSO}_{4}$ and the solvents were evaporated under reduced pressure. The crude product was purified by column chromatography (silica gel, Hex/EtOAc).

\section{1-(4-tert-Butylbenzyl)-4-(4-methoxyphenyl)-1H-1,2,3-triazole (1b)}

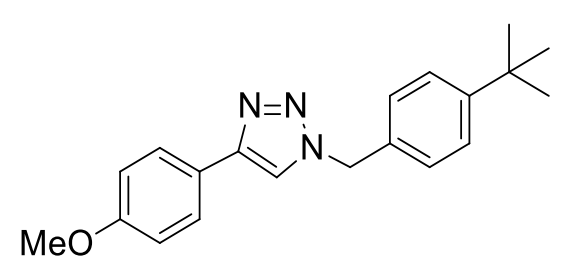

The general procedure was followed starting from 4tert-butylbenzyl azide (1.00 mmol, $189 \mathrm{mg})$, 4ethynylanisole $(1.10 \mathrm{mmol}, 132 \mu \mathrm{L}), \mathrm{CuSO}_{4} \cdot 5 \mathrm{H}_{2} \mathrm{O}(0.40$ $\mathrm{mmol}, 100 \mathrm{mg}$ ) and sodium ascorbate (0.80 mmol, 158 $\mathrm{mg}$ ) in $\mathrm{THF} /{ }^{\mathrm{BuOH}} / \mathrm{H}_{2} \mathrm{O}$ 1:1:1 (6 mL). Yield: $288 \mathrm{mg}$

$(90 \%)$. White solid $\left(\mathrm{mp}=118-120{ }^{\circ} \mathrm{C}\right) .{ }^{1} \mathrm{H}$ NMR $\left(400 \mathrm{MHz}, \mathrm{CDCl}_{3}\right): \delta 7.74(\mathrm{~d}, J=8.2$ $\mathrm{Hz}, 2 \mathrm{H}), 7.63(\mathrm{~s}, 1 \mathrm{H}), 7.40(\mathrm{~d}, J=7.9 \mathrm{~Hz}, 2 \mathrm{H}), 7.25(\mathrm{~d}, J=7.9 \mathrm{~Hz}, 2 \mathrm{H}), 6.94(\mathrm{~d}, J=8.2$ $\mathrm{Hz}, 2 \mathrm{H}), 5.51(\mathrm{~s}, 2 \mathrm{H}), 3.81(\mathrm{~s}, 3 \mathrm{H}), 1.33$ (s, 9H). ${ }^{13} \mathrm{C} \mathrm{NMR}\left(101 \mathrm{MHz}, \mathrm{CDCl}_{3}\right): \delta 159.7$, $151.9,148.1,132.0,128.0,127.1,126.1,123.5,119.0,114.3,55.4,54.0,34.8,31.4$. IR $\left(\mathrm{cm}^{-1}\right): 1454,1250,1219$. HRMS (ESI+): $\mathrm{m} / z[\mathrm{M}+\mathrm{H}]^{+}$calcd for $\mathrm{C}_{20} \mathrm{H}_{24} \mathrm{~N}_{3} \mathrm{O}: 322.1919$; found: 322.1924 .

\section{1-Benzyl-4-\{2-[N,N'-di(tert-butoxycarbonyl)-guanidyl]-ethyl $\}-1 H-1,2,3$-triazole (1f)}<smiles>CC(C)(C)OC(=O)NC(=NC(=O)OCc1ccccc1)NCCc1cn(Cc2ccccc2)nn1</smiles>

A suspension of $N, N^{\prime}$-di-Boc- $N^{\prime \prime}$-(but-3-ynyl)guanidine (1.61 mmol, $500 \mathrm{mg})$, benzyl azide (1.61 mmol, $214 \mathrm{mg})$, CuI (1.61 mol, $306 \mathrm{mg})$ and DIPEA $(1.61 \mathrm{mmol}, 281 \mu \mathrm{L})$ in anhydrous $\mathrm{MeCN}(10 \mathrm{~mL})$ was stirred under nitrogen atmosphere at ambient temperature for 24 hours. The solvent was evaporated under reduced pressure, 
$\mathrm{CH}_{2} \mathrm{Cl}_{2}$ was added and the product was washed with a saturated aqueous solution of $\mathrm{NH}_{4} \mathrm{Cl}$. The organic layer was dried over $\mathrm{MgSO}_{4}$, the solvent was evaporated and the resulting crude product was purified by column chromatography (silica gel, Hex/EtOAc 1:1). Yield: $633 \mathrm{mg}(89 \%)$. White solid $\left(\mathrm{mp}=139-140{ }^{\circ} \mathrm{C}\right) .{ }^{1} \mathrm{H}$ NMR (400 $\left.\mathrm{MHz}, \mathrm{CDCl}_{3}\right)$ : ठ $11.48(\mathrm{~s}, 1 \mathrm{H}), 8.59(\mathrm{~s}, 1 \mathrm{H}), 7.49-7.22(\mathrm{~m}, 6 \mathrm{H}), 5.53(\mathrm{~s}, 2 \mathrm{H}), 3.79(\mathrm{q}, J=6.5 \mathrm{~Hz}, 2 \mathrm{H})$, $3.01(\mathrm{t}, J=6.9 \mathrm{~Hz}, 2 \mathrm{H}), 1.52(\mathrm{~s}, 9 \mathrm{H}), 1.51(\mathrm{~s}, 9 \mathrm{H}) .{ }^{13} \mathrm{C} \mathrm{NMR}\left(101 \mathrm{MHz}, \mathrm{CDCl}_{3}\right): \delta 163.0$, $155.6,152.4,144.5,134.5,128.4,128.0,127.3,121.2,82.4,78.4,53.3,39.5,27.7,27.4$, 25.0. IR $\left(\mathrm{cm}^{-1}\right): 3328,1714,1153,1130$. HRMS (ESI+): $m / z[\mathrm{M}+\mathrm{H}]^{+}$calcd for $\mathrm{C}_{22} \mathrm{H}_{33} \mathrm{~N}_{6} \mathrm{O}_{4}$ : 445.2563; found: 445.2570 .

\section{(S)-4-\{3-[N, $N$ '-Di(tert-butoxycarbonyl)-guanidyl]-propyl $\}-5-i o d o-1-\{N$-[1-phenyl-2- (methoxycarbonyl)ethyl]carbamoylmethyl\}-1H-1,2,3-triazole (1g)}<smiles>COC(=O)CC[C@H](NC(=O)Cn1nnc(CCCNC(=NC(=O)OCc2ccccc2)NC(=O)OCc2ccccc2)c1I)c1ccccc1</smiles>

A suspension of $N, N^{\prime}$-di-Boc- $N$ ' '-(pent-4ynyl)-guanidine (0.15 mmol, $50 \mathrm{mg}),(3 R)$ -

(2-azido-acetylamino)-3-phenyl-propionic acid methyl ester (0.15 mmol, $40 \mathrm{mg})$, DIPEA $(0.17 \mathrm{mmol}, 30 \mu \mathrm{L}), \mathrm{CuI}(0.17$ mmol, $32 \mathrm{mg}$ ) and $N$-bromosuccinimide $(0.18 \mathrm{mmol}, 33 \mathrm{mg})$ in anhydrous $\mathrm{MeCN}(0.40$ $\mathrm{mL}$ ) was stirred under nitrogen atmosphere at ambient temperature for 4 hours. The solvent was evaporated under reduced pressure and the resulting product was purified by column chromatography (silica gel, Hex/EtOAc 1:4). Yield: $65 \mathrm{mg}(58 \%)$. Brown solid (mp = 68$\left.70{ }^{\circ} \mathrm{C}\right) .[\alpha]_{\mathrm{D}}{ }^{22}=-10.98^{\circ}$ (c. $\left.1.10, \mathrm{CH}_{2} \mathrm{Cl}_{2}\right) .{ }^{1} \mathrm{H} \mathrm{NMR}\left(500 \mathrm{MHz}, \mathrm{CDCl}_{3}\right): \delta 11.50(\mathrm{~s}, 1 \mathrm{H})$, $8.44(\mathrm{~s}, 1 \mathrm{H}), 7.34-7.18(\mathrm{~m}, 5 \mathrm{H}), 7.06(\mathrm{~d}, J=8.3 \mathrm{~Hz}, 1 \mathrm{H}), 5.40(\mathrm{~m}, 1 \mathrm{H}), 5.11(\mathrm{~s}, 2 \mathrm{H}), 3.58$ $(\mathrm{s}, 3 \mathrm{H}), 3.55-3.46(\mathrm{~m}, 2 \mathrm{H}), 2.90-2.71(\mathrm{~m}, 4 \mathrm{H}), 2.08-2.00(\mathrm{~m}, 2 \mathrm{H}), 1.51(\mathrm{~s}, 9 \mathrm{H}), 1.50(\mathrm{~s}$, $9 \mathrm{H}) .{ }^{13} \mathrm{C}$ NMR $\left(126 \mathrm{MHz}, \mathrm{CDCl}_{3}\right): \delta 171.2,163.9,163.4,156.1,153.2,151.6,139.6$, $128.7,127.8,126.1,83.1,80.1$, 79.3 , 53.2, 51.9, 49.8, 40.0, 39.4, 29.5, 28.2, 28.0, 23.5. IR $\left(\mathrm{cm}^{-1}\right): 3323,2979,1718,615,557$. HRMS (ESI+): $\mathrm{m} / z[\mathrm{M}+\mathrm{H}]^{+}$calcd for $\mathrm{C}_{28} \mathrm{H}_{41} \mathrm{~N}_{7} \mathrm{O}_{7} \mathrm{I}$ : 714.2112; found: 714.2105 .

\section{4-Butyl-1-(pivaloyloxymethyl)-1H-1,2,3-triazole (1j)}

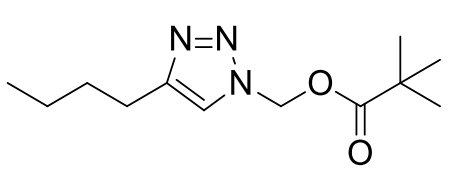

The general procedure was followed starting from azidomethyl pivalate $(12.3 \mathrm{mmol}, 1.90 \mathrm{~g})$, hept-1-yne (13.50 mmol, $1.55 \mathrm{~mL}), \mathrm{CuSO}_{4} \cdot 5 \mathrm{H}_{2} \mathrm{O}(0.61 \mathrm{mmol}, 181 \mathrm{mg})$ and sodium ascorbate $\left(3.70 \mathrm{mmol}, 732 \mathrm{mg}\right.$ ) in $\mathrm{THF} /{ }^{t} \mathrm{BuOH} / \mathrm{H}_{2} \mathrm{O}$ 
1:1:1 (30 mL). Yield: $1.9(66 \%)$. Yellowish oil. ${ }^{1} \mathrm{H}$ NMR (400 MHz, $\left.\mathrm{CDCl}_{3}\right): \delta 7.46(\mathrm{~s}$, $1 \mathrm{H}), 6.10(\mathrm{~s}, 2 \mathrm{H}), 2.60(\mathrm{t}, J=7.7 \mathrm{~Hz}, 2 \mathrm{H}), 1.54(\mathrm{p}, J=7.6 \mathrm{~Hz}, 2 \mathrm{H}), 1.25$ (q, J = $7.6 \mathrm{~Hz}$, $2 \mathrm{H}), 1.06(\mathrm{~s}, 9 \mathrm{H}), 0.80(\mathrm{t}, J=7.4 \mathrm{~Hz}, 3 \mathrm{H}) .{ }^{13} \mathrm{C} \mathrm{NMR}\left(101 \mathrm{MHz}, \mathrm{CDCl}_{3}\right): \delta 177.5,148.7$, $121.8,69.5,38.6,31.2,26.6,25.0,22.0,13.6$. IR $\left(\mathrm{cm}^{-1}\right): 2960,1744,1458,1243,1118$, 1031, 776. HRMS (ESI+): $m / z[\mathrm{M}+\mathrm{H}]^{+}$calcd for $\mathrm{C}_{12} \mathrm{H}_{22} \mathrm{~N}_{3} \mathrm{O}_{2}: 240.1712$; found: 240.1720 .

\section{4-Ethynyl-1-(pivaloyloxymethyl)-1H-1,2,3-triazole (1k)}

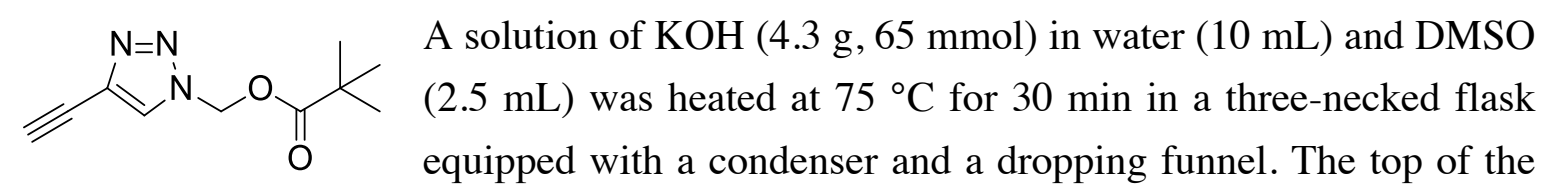
condenser was connected, via a tube, to a trap containing dry THF, which was cooled at $20{ }^{\circ} \mathrm{C}$. 1,4-Dichloro-2-butyne $(1.60 \mathrm{~mL}, 16 \mathrm{mmol})$ was added dropwise over a period of 30 min, while the temperature was maintained at $75^{\circ} \mathrm{C}$. A stream of argon was passed through the apparatus, which forced the butadiyne into the cold THF trap. The tube was extracted, and azidomethyl pivalate $(512 \mathrm{mg}, 3.3 \mathrm{mmol})$, sodium acetate $(812 \mathrm{mg}, 9.90$ mmol) and CuOAc (120 mg, $0.66 \mathrm{mmol})$ were added and the mixture was stirred at ambient temperature for 3-4 hours. Then, the solution was filtered through a celite pad, and the solvents were evaporated under reduced pressure. The crude product was purified by column chromatography (silica gel, Hex/EtOAc 7:3). Yield: $301 \mathrm{mg}$ (44\%). White solid $\left(\mathrm{mp}=173-195^{\circ} \mathrm{C}\right) .{ }^{1} \mathrm{H}$ NMR $\left(400 \mathrm{MHz}, \mathrm{CDCl}_{3}\right): \delta 8.06(\mathrm{~s}, 1 \mathrm{H}), 6.31(\mathrm{~s}, 2 \mathrm{H}), 3.35(\mathrm{~s}, 1 \mathrm{H})$, 1.25 (s, 9H). ${ }^{13} \mathrm{C} \mathrm{NMR}\left(126 \mathrm{MHz}, \mathrm{CDCl}_{3}\right): \delta 177.7,140.3,122.2,75.6,69.9,38.9,27.0 . \mathrm{IR}$ $\left(\mathrm{cm}^{-1}\right): 2976,1730,1279,1144,1089,994,829,727$. HRMS (ESI+): $m / z[\mathrm{M}+\mathrm{H}]^{+}$calcd for $\mathrm{C}_{10} \mathrm{H}_{14} \mathrm{~N}_{3} \mathrm{O}_{2}$ : 208.1086; found: 208.1096.

\subsection{Synthesis of $\mathrm{N}$-alkyl-1H-1,2,3-triazolium trifluoromethanesulfonate}

General Procedure: To a solution of the corresponding triazole $(1.00 \mathrm{mmol})$ in $\mathrm{CH}_{2} \mathrm{Cl}_{2}$ $(8.6 \mathrm{~mL})$ the selected alkyl trifluoromethanesulfonate $(1.20 \mathrm{mmol})$ was added and the resulting solution was slowly evaporated under reduced pressure to form a homogeneous thick solution. After 18 hours at $30^{\circ} \mathrm{C}$ the product was purified by column chromatography (silica gel, $\mathrm{CH}_{2} \mathrm{Cl}_{2} / \mathrm{MeOH}$ 95:5). 


\section{1-Benzyl-4-phenyl-3-[2-(2,4,6-triiodophenoxy)ethyl]-1H-1,2,3-triazolium trifluoromethanesulfonate (2a)}

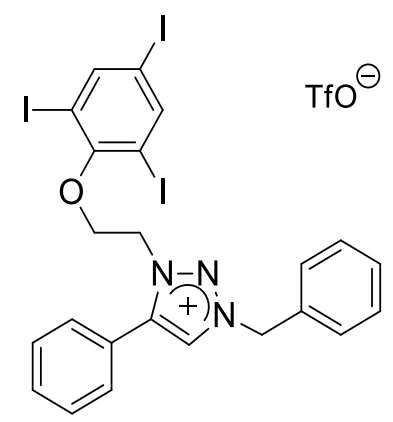

The general procedure was followed starting from 1-benzyl-4phenyl- $1 H$-1,2,3-triazole $1 \mathrm{a}(0.03 \mathrm{mmol}, 8 \mathrm{mg})$ and 2-(2,4,6triiodophenoxy)ethyl trifluoromethanesulfonate $(0.04 \mathrm{mmol}, 25$ $\mathrm{mg})$. Yield: $29 \mathrm{mg}(98 \%)$. White solid $\left(\mathrm{mp}=67-68{ }^{\circ} \mathrm{C}\right) .{ }^{1} \mathrm{H} \mathrm{NMR}$ $\left(400 \mathrm{MHz}, \mathrm{CDCl}_{3}\right): \delta 8.94(\mathrm{~s}, 1 \mathrm{H}), 7.96(\mathrm{~s}, 2 \mathrm{H}), 7.76(\mathrm{~d}, J=7.4$ $\mathrm{Hz}, 2 \mathrm{H}), 7.70-7.48(\mathrm{~m}, 5 \mathrm{H}), 7.48-7.37(\mathrm{~m}, 3 \mathrm{H}), 5.92(\mathrm{~s}, 2 \mathrm{H}), 5.02$ $(\mathrm{t}, J=4.9 \mathrm{~Hz}, 2 \mathrm{H}), 4.36(\mathrm{t}, J=5.2 \mathrm{~Hz}, 2 \mathrm{H}) .{ }^{13} \mathrm{C}$ NMR $(101 \mathrm{MHz}$, $\left.\mathrm{CDCl}_{3}\right): \delta 155.6,147.2,144.9,131.8,131.5,130.2,130.1,130.0,129.5,128.83,121.7$, 91.5, 90.6, 68.8, 57.9, 50.9. IR ( $\left.\mathrm{cm}^{-1}\right): 1457,1251,1223,1151,1027,766,730,698,635$. HRMS (ESI+): $m / z$ [M] $]^{+}$calcd for $\mathrm{C}_{23} \mathrm{H}_{19} \mathrm{~N}_{3} \mathrm{OI}_{3}:$ : 733.8662; found: 733.8679 .

\section{1-Benzyl-4-phenyl-3-\{2-[2-(2,4,6-triiodophenoxy)ethoxy]ethyl\}-1H-1,2,3-triazolium trifluoromethanesulfonate (2b)}

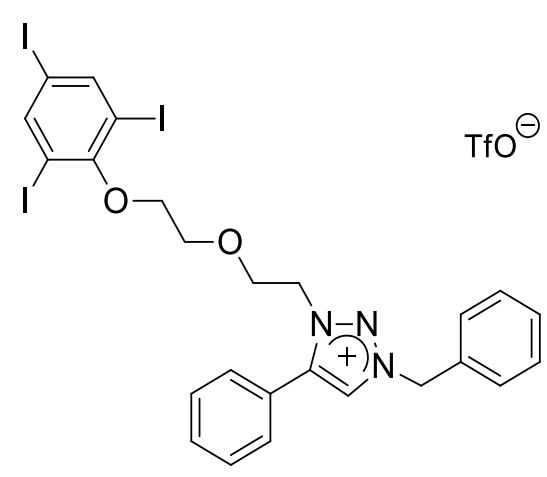

The general procedure was followed starting from 1benzyl-4-phenyl-1H-1,2,3-triazole $1 \mathbf{a}(0.04 \mathrm{mmol}, 10$ $\mathrm{mg})$ and 2-[2-(2,4,6-triiodophenoxy)ethoxy]ethyl trifluoromethanesulfonate $(0.05 \mathrm{mmol}, 32 \mathrm{mg})$. Yield: $30 \mathrm{mg}(77 \%)$. Waxy solid. ${ }^{1} \mathrm{H}$ NMR (400 $\left.\mathrm{MHz}, \mathrm{CDCl}_{3}\right)$ : ठ $8.64(\mathrm{~s}, 1 \mathrm{H}), 8.01(\mathrm{~s}, 2 \mathrm{H}), 7.68(\mathrm{~d}, J=7.4 \mathrm{~Hz}, 2 \mathrm{H})$, 7.64-7.47 (m, 5H), 7.46-7.36 (m, 3H), $5.91(\mathrm{~s}, 2 \mathrm{H}), 4.77$ $(\mathrm{t}, J=5.2 \mathrm{~Hz}, 2 \mathrm{H}), 4.19(\mathrm{t}, J=5.2 \mathrm{~Hz}, 2 \mathrm{H}), 4.04(\mathrm{t}, J=$ $4.4 \mathrm{~Hz}, 2 \mathrm{H}), 3.90(\mathrm{t}, J=4.4 \mathrm{~Hz}, 2 \mathrm{H}) .{ }^{13} \mathrm{C} \mathrm{NMR}\left(101 \mathrm{MHz}, \mathrm{CDCl}_{3}\right): \delta 157.6,147.3,144.4$, $131.8,131.1,130.1,130.0,129.7,129.6,129.5,128.4,121.8,91.8,89.5,72.1,70.5,68.0$, 57.9, 51.5. IR ( $\left.\mathrm{cm}^{-1}\right): 1452,1252,1223,1150,1027,765,736,697,635$. HRMS (ESI+): $m / z[\mathrm{M}]^{+}$calcd for $\mathrm{C}_{25} \mathrm{H}_{23} \mathrm{~N}_{3} \mathrm{O}_{2} \mathrm{I}_{3}: 777.8924$; found: 777.8936 .

\section{3-(2-Azidoethyl)-1-benzyl-4-phenyl-1H-1,2,3-triazolium trifluoromethanesulfonate (2c)}

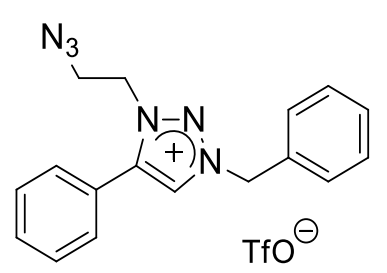

The general procedure was followed starting from 1-benzyl-4phenyl-1 $H$-1,2,3-triazole 1a $(0.14 \mathrm{mmol}, 34 \mathrm{mg})$ and 2-azidoethyl trifluoromethanesulfonate $\mathbf{1 6}(0.16 \mathrm{mmol}, 35 \mathrm{mg})$. Yield: $30 \mathrm{mg}$ 
(46 \%). Colorless oil. ${ }^{1} \mathrm{H}$ NMR (400 $\left.\mathrm{MHz} \mathrm{CDCl}_{3}\right): \delta 8.58(\mathrm{~s}, 1 \mathrm{H}), 7.70-7.50(\mathrm{~m}, 7 \mathrm{H})$, $7.50-7.41(\mathrm{~m}, 3 \mathrm{H}), 5.86(\mathrm{~s}, 2 \mathrm{H}), 4.68(\mathrm{t}, J=5.7 \mathrm{~Hz}, 2 \mathrm{H}), 3.97(\mathrm{t}, J=5.7 \mathrm{~Hz}, 2 \mathrm{H}) .{ }^{13} \mathrm{C} \mathrm{NMR}$ $\left(101 \mathrm{MHz}, \mathrm{CDCl}_{3}\right): \delta 144.2,132.0,130.8,130.1,129.7,129.6,129.5,128.5,121.4,58.0$, 50.6, 48.7. IR $\left(\mathrm{cm}^{-1}\right): 2104,1457,1255,1224,1151,1029,766,739,700,636$. HRMS (ESI+): $m / z[\mathrm{M}]^{+}$calcd for $\mathrm{C}_{17} \mathrm{H}_{17} \mathrm{~N}_{6}: 305.1515$; found: 305.1524 .

\section{3-(3-Azidopropyl)-1-benzyl-4-phenyl-1H-1,2,3-triazolium trifluoromethanesulfonate (2d)}

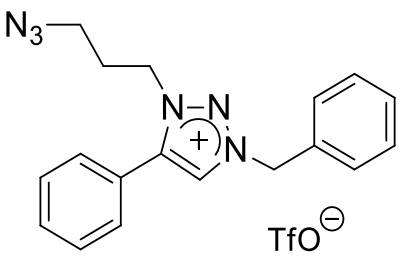

The general procedure was followed starting from 1-benzyl-4phenyl-1H-1,2,3-triazole 1a $(0.14 \mathrm{mmol}, 34 \mathrm{mg})$ and 3 azidopropyl trifluoromethanesulfonate $14(0.16 \mathrm{mmol}, 37 \mathrm{mg})$. Yield: $40 \mathrm{mg}(59 \%)$. Colorless oil. ${ }^{1} \mathrm{H}$ NMR (400 MHz, $\left.\mathrm{CDCl}_{3}\right): \delta 8.56(\mathrm{~s}, 1 \mathrm{H}), 7.73-7.33(\mathrm{~m}, 10 \mathrm{H}), 5.84(\mathrm{~s}, 2 \mathrm{H}), 4.62$ $(\mathrm{t}, J=6.8 \mathrm{~Hz}, 2 \mathrm{H}), 3.42(\mathrm{t}, J=6.2 \mathrm{~Hz}, 2 \mathrm{H}), 2.32-2.07(\mathrm{~m}, 2 \mathrm{H}) .{ }^{13} \mathrm{C} \mathrm{NMR}(101 \mathrm{MHz}$, $\left.\mathrm{CDCl}_{3}\right): \delta 143.4,131.9,131.0,130.0,129.7,129.5,129.4,128.6,121.6,57.8,49.0,47.7$, 27.9. IR ( $\left.\mathrm{cm}^{-1}\right): 2100,1457,1256,1224,1152,1029,766,736,700,636$. HRMS (ESI+): $m / z[\mathrm{M}]^{+}$calcd for $\mathrm{C}_{18} \mathrm{H}_{19} \mathrm{~N}_{6}: 319.1671$; found: 319.1682 .

\section{1-Benzyl-3-butyl-4-phenyl-1H-1,2,3-triazolium trifluoromethanesulfonate (2e)}

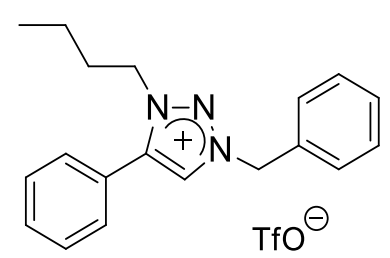

The general procedure was followed starting from 1-benzyl-4phenyl-1H-1,2,3-triazole $1 \mathbf{a}(0.14 \mathrm{mmol}, 34 \mathrm{mg})$ and butyl trifluoromethanesulfonate $(0.16 \mathrm{mmol}, 33 \mathrm{mg})$. Yield: $33 \mathrm{mg}(52$ \%). Colorless oil. ${ }^{1} \mathrm{H}$ NMR (400 $\left.\mathrm{MHz}, \mathrm{CDCl}_{3}\right): \delta 8.71(\mathrm{~s}, 1 \mathrm{H})$, 7.77-7.35 (m, 10H), $5.86(\mathrm{~s}, 2 \mathrm{H}), 4.51(\mathrm{t}, J=7.5 \mathrm{~Hz}, 2 \mathrm{H}), 2.08-$ $1.76(\mathrm{~m}, 2 \mathrm{H}), 1.44-1.11(\mathrm{~m}, 2 \mathrm{H}), 0.88(\mathrm{t}, J=7.5 \mathrm{~Hz}, 3 \mathrm{H}) .{ }^{13} \mathrm{C} \mathrm{NMR}\left(126 \mathrm{MHz}, \mathrm{CDCl}_{3}\right): \delta$ 143.2, 132.0, 131.4, 130.0, 129.8, 129.7, 129.6, 129.5, 128.9, 122.0, 57.8, 51.7, 30.9, 19.4, 13.2. IR $\left(\mathrm{cm}^{-1}\right): 1458,1256,1223,1152,1029,637$. HRMS (ESI+): $\mathrm{m} / z[\mathrm{M}]^{+}$calcd for $\mathrm{C}_{19} \mathrm{H}_{22} \mathrm{~N}_{3}$ : 292.1814; found: 292.1826 .

\section{1-Benzyl-4-phenyl-3-(4-pentyn-1-yl)-1H-1,2,3-triazolium trifluoromethanesulfonate (2f)}

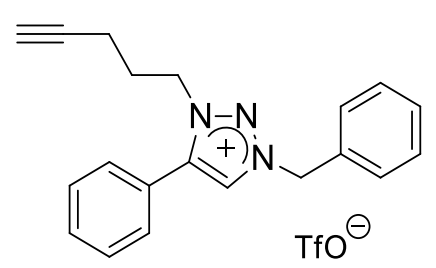

The general procedure was followed starting from 1-benzyl-4phenyl-1H-1,2,3-triazole 1a $(0.14 \mathrm{mmol}, 34 \mathrm{mg})$ and hex-5-yn1-yl trifluoromethanesulfonate $(0.16 \mathrm{mmol}, 35 \mathrm{mg})$. Yield: 50 mg $(76 \%)$. Colorless oil. ${ }^{1} \mathrm{H}$ NMR (400 $\left.\mathrm{MHz} \mathrm{CDCl}_{3}\right): \delta 8.67$ (s, 1H), 7.79-7.36 (m, 10H), $5.87(\mathrm{~s}, 2 \mathrm{H}), 4.68(\mathrm{t}, J=6.9 \mathrm{~Hz}$, 
2H), 2.34-2.25 (m, 2H), 2.22-2.11 (m, 2H), $1.85(\mathrm{~s}, 1 \mathrm{H}) .{ }^{13} \mathrm{C}$ NMR (101 $\left.\mathrm{MHz}, \mathrm{CDCl}_{3}\right): \delta$ 143.6, 131.9, 131.2 , 130.0, 129.8, 129.7, 129.6, 129.5, 128.8, 121.7, 80.7 , 70.4, 57.8, 50.6, 27.1, 15.5. IR $\left(\mathrm{cm}^{-1}\right): 1458,1256,1223,1152,1030,700,637$. HRMS (ESI+): $\mathrm{m} / z[\mathrm{M}]^{+}$ calcd for $\mathrm{C}_{20} \mathrm{H}_{20} \mathrm{~N}_{3}$ : 302.1657; found: 302.1670.

\section{1-(4-tert-Butylbenzyl)-4-(4-methoxyphenyl)-3-(4-pentyn-1-yl)-1H-1,2,3-triazolium trifluoromethanesulfonate $(2 \mathrm{~g})$}

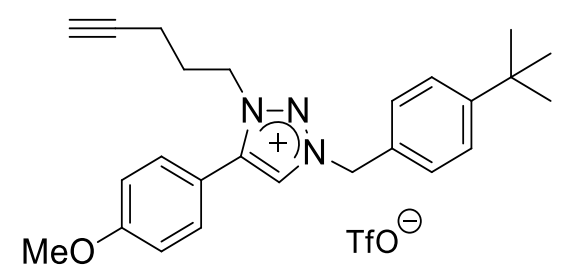

The general procedure was followed starting from 1-(4tert-butylbenzyl)-4-(4-metoxyphenyl)-1H-1,2,3-triazole $1 b(0.16 \mathrm{mmol}, 50 \mathrm{mg})$ and hex-5-yn-1-yl trifluoromethanesulfonate $(0.18 \mathrm{mmol}, 38 \mathrm{mg})$. Yield: $70 \mathrm{mg}$ (81\%). Colorless oil. ${ }^{1} \mathrm{H}$ NMR (400 $\left.\mathrm{MHz}, \mathrm{CDCl}_{3}\right): \delta$ $8.61(\mathrm{~s}, 1 \mathrm{H}), 7.64-7.41(\mathrm{~m}, 6 \mathrm{H}), 7.03(\mathrm{~d}, J=8.4 \mathrm{~Hz}, 2 \mathrm{H}), 5.79(\mathrm{~s}, 2 \mathrm{H}), 4.67(\mathrm{t}, J=6.8 \mathrm{~Hz}$, $2 \mathrm{H}), 3.84(\mathrm{~s}, 3 \mathrm{H}), 2.36-2.18(\mathrm{~m}, 2 \mathrm{H}), 2.21-2.08(\mathrm{~m}, 2 \mathrm{H}), 1.87(\mathrm{~s}, 1 \mathrm{H}), 1.31(\mathrm{~s}, 9 \mathrm{H}) .{ }^{13} \mathrm{C}$ NMR $\left(101 \mathrm{MHz} \mathrm{CDCl}_{3}\right): \delta 162.5,153.5,143.8,131.3,129.8,129.7,128.5,126.7,120.4$ $(\mathrm{q}, J=320.0 \mathrm{~Hz}), 115.5,113.8,81.1,70.6,57.7,55.8,50.8,35.0,31.4,27.3,15.8$. IR $\left(\mathrm{cm}^{-}\right.$ $\left.{ }^{1}\right): 1461,1254,1223,1180,1029,637$. HRMS (ESI+): $m / z[\mathrm{M}]^{+}$calcd for $\mathrm{C}_{25} \mathrm{H}_{30} \mathrm{~N}_{3} \mathrm{O}$ : 388.2389; found: 388.2399.

\section{1-Benzyl-5-iodo-4-phenyl-3-[2-(2,4,6-triiodophenoxy)ethyl]-1H-1,2,3-triazolium trifluoromethanesulfonate $(2 \mathrm{~h})$}

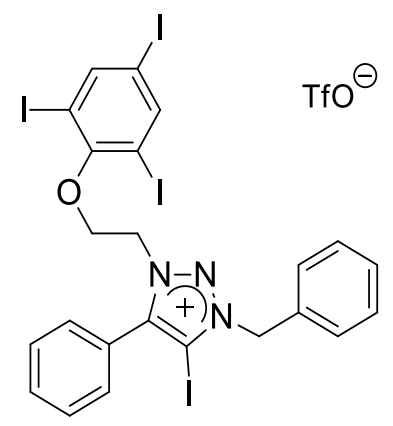

The general procedure was followed starting from 1-benzyl-5iodo-4-phenyl-1H-1,2,3-triazole 1c $(0.03 \mathrm{mmol}, 12 \mathrm{mg})$ and 2(2,4,6-triiodophenoxy)ethyl trifluoromethanesulfonate $(0.04$ mmol, $25 \mathrm{mg})$. Yield: $31 \mathrm{mg}(91 \%)$. White solid $\left(\mathrm{mp}=80-83{ }^{\circ} \mathrm{C}\right)$. ${ }^{1} \mathrm{H} \mathrm{NMR}\left(400 \mathrm{MHz} \mathrm{CDCl}_{3}\right.$ ): $\delta 7.96(\mathrm{~s}, 2 \mathrm{H}), 7.71(\mathrm{~d}, J=7.3 \mathrm{~Hz}$, 2H), 7.66-7.52 (m, 5H), 7.51-7.39 (m, 3H) $5.85(\mathrm{~s}, 2 \mathrm{H}), 5.04(\mathrm{t}, J$ $=5.0 \mathrm{~Hz}, 2 \mathrm{H}), 4.30(\mathrm{t}, J=5.0 \mathrm{~Hz}, 2 \mathrm{H}) \cdot{ }^{13} \mathrm{C} \mathrm{NMR}(101 \mathrm{MHz}$, $\left.\mathrm{CDCl}_{3}\right): \delta 156.0,148.9,147.6,132.3,131.6,131.2,130.4,130.1,129.9,129.8,122.7,91.7$, 90.9, 89.8, 68.9, 58.8, 52.4. IR $\left(\mathrm{cm}^{-1}\right): 1455,1235,1152,1026,699,635$. HRMS (ESI+): $m / z[\mathrm{M}]^{+}$calcd for $\mathrm{C}_{23} \mathrm{H}_{18} \mathrm{~N}_{3} \mathrm{OI}_{4}: 859.7629$; found: 859.7649 . 
1-Benzyl-4-ethynyl-3-[2-(2,4,6-triiodophenoxy)ethyl]-1H-1,2,3-triazolium trifluoromethanesulfonate (2i)

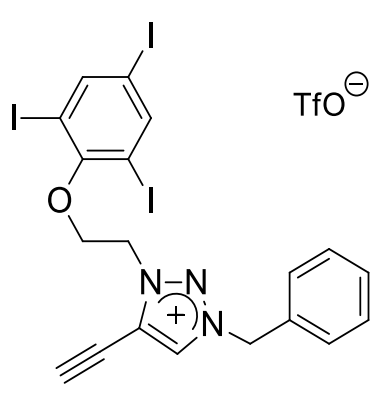

The general procedure was followed starting from 1-benzyl-4ethynyl- $1 \mathrm{H}-1,2,3$-triazole $\mathbf{1 d}(0.05 \mathrm{mmol}, 9 \mathrm{mg})$ and 2-(2,4,6triiodophenoxy)ethyl trifluoromethanesulfonate $(0.06 \mathrm{mmol}, 36$ $\mathrm{mg})$. Yield: $35 \mathrm{mg}(81 \%)$. White solid $\left(\mathrm{mp}=154-157{ }^{\circ} \mathrm{C}\right) .{ }^{1} \mathrm{H}$ NMR (400 MHz, $\left.\mathrm{CDCl}_{3}\right): \delta 9.15(\mathrm{~s}, 1 \mathrm{H}), 7.97(\mathrm{~s}, 2 \mathrm{H}), 7.76-7.54$ $(\mathrm{m}, 2 \mathrm{H}), 7.54-7.36(\mathrm{~m}, 3 \mathrm{H}), 5.93(\mathrm{~s}, 2 \mathrm{H}), 5.16(\mathrm{t}, J=5.0 \mathrm{~Hz}, 2 \mathrm{H})$, $4.38(\mathrm{t}, J=5.1 \mathrm{~Hz}, 2 \mathrm{H}), 4.13(\mathrm{~s}, 1 \mathrm{H}) .{ }^{13} \mathrm{C}$ NMR $\left(101 \mathrm{MHz}, \mathrm{CDCl}_{3}\right)$ : ठ $155.5,147.3,133.0,131.1,130.3,129.9,129.6,127.2,120.6$ (q, $J=320.2 \mathrm{~Hz}), 94.4$, 91.5, 90.6, 68.2, 64.5, 58.4, 52.3. IR ( $\left.\mathrm{cm}^{-1}\right): 2130,1428,1248,1223,1154,1049,729,702$, 635. HRMS (ESI+): $\mathrm{m} / z$ [M] ${ }^{+}$calcd for $\mathrm{C}_{19} \mathrm{H}_{15} \mathrm{~N}_{3} \mathrm{OI}_{3}: 681.8349$; found: 681.8340 .

1-Methoxycarbonylmethyl-4-phenyl-3-[2-(2,4,6-triiodophenoxy)ethyl]-1H-1,2,3triazolium trifluoromethanesulfonate $(2 \mathrm{j})$

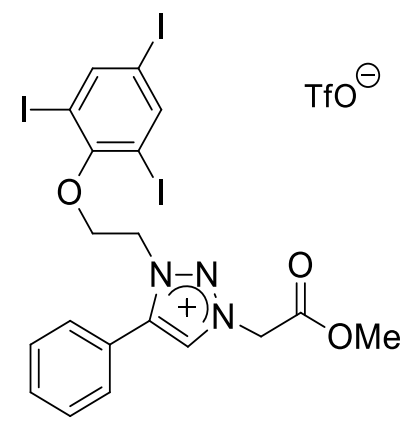

The general procedure was followed starting from 1methoxycarbonylmethyl-4-phenyl- $1 H$-1,2,3-triazole $\quad$ 1e $\quad(0.03$ mmol, $7 \mathrm{mg}$ ) and 2-(2,4,6-triiodophenoxy)ethyl trifluoromethanesulfonate $(0.04 \mathrm{mmol}, 25 \mathrm{mg})$. Yield: $23 \mathrm{mg}(79 \%)$. White solid $\left(\mathrm{mp}=52-54{ }^{\circ} \mathrm{C}\right) .{ }^{1} \mathrm{H}$ NMR $\left(400 \mathrm{MHz}, \mathrm{CDCl}_{3}\right): \delta 8.87(\mathrm{~s}$, $1 \mathrm{H}), 8.04(\mathrm{~s}, 2 \mathrm{H}), 7.81(\mathrm{~d}, J=7.4 \mathrm{~Hz}, 2 \mathrm{H}), 7.70-7.54(\mathrm{~m}, 3 \mathrm{H})$, $5.76(\mathrm{~s}, 2 \mathrm{H}), 5.04(\mathrm{t}, J=5.0 \mathrm{~Hz}, 2 \mathrm{H}), 4.43(\mathrm{t}, J=5.0 \mathrm{~Hz}, 2 \mathrm{H})$, 3.90 (s, 3H). ${ }^{13} \mathrm{C} \mathrm{NMR} \mathrm{(101} \mathrm{MHz,} \mathrm{CDCl}_{3}$ ): $\delta$ 165.6, 155.9, 147.7, 145.3, 132.4, 131.3, 130.6, 130.0, 121.9, 91.9, 91.1, 69.3, 54.5, 54.1, 51.5. IR $\left(\mathrm{cm}^{-1}\right): 1751,1456,1223,1153$, 1027, 674, 635. HRMS (ESI+): $m / z[\mathrm{M}]^{+}$calcd for $\mathrm{C}_{19} \mathrm{H}_{17} \mathrm{~N}_{3} \mathrm{O}_{3} \mathrm{I}_{3}:$ 715.8404; found: 715.8411 . 


\section{1-Benzyl-4-\{2-[N,N'-di(tert-butoxycarbonyl)guanidyl]ethyl\}-3-[2-(2,4,6-triiodophe- noxy)ethyl]-1H-1,2,3-triazolium trifluoromethanesulfonate (2k)}

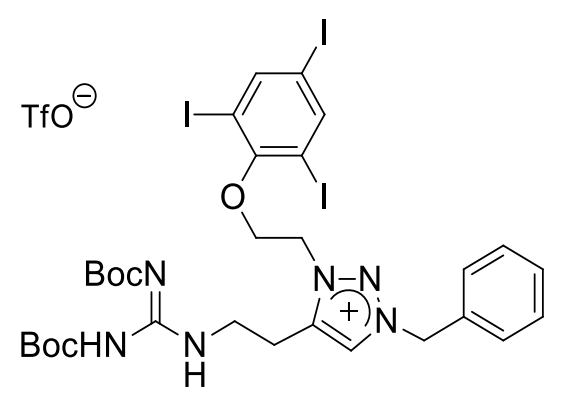

The general procedure was followed starting from 1benzyl-4-\{2-[N,N'-di(tert-butoxycarbonyl)guanidyl]ethyl $\}$ - $1 H-1,2,3$-triazole $\mathbf{1 f}(0.03 \mathrm{mmol}, 15 \mathrm{mg})$ and 2(2,4,6-triiodophenoxy)ethyl trifluoromethanesulfonate (0.04 mmol, $25 \mathrm{mg})$. Yield: $34 \mathrm{mg}(92 \%)$. White solid $\left(\mathrm{mp}=73-74{ }^{\circ} \mathrm{C}\right) .{ }^{1} \mathrm{H}$ NMR $\left(400 \mathrm{MHz}, \mathrm{CDCl}_{3}\right): \delta 11.23$ $(\mathrm{s}, 1 \mathrm{H}), 8.98(\mathrm{~s}, 1 \mathrm{H}), 8.70(\mathrm{~s}, 1 \mathrm{H}), 7.98(\mathrm{~s}, 2 \mathrm{H}), 7.67-$ $7.51(\mathrm{~m}, 2 \mathrm{H}), 7.50-7.35(\mathrm{~m}, 3 \mathrm{H}), 5.83(\mathrm{~s}, 2 \mathrm{H}), 5.39(\mathrm{t}, J=4.2 \mathrm{~Hz}, 2 \mathrm{H}), 4.37(\mathrm{t}, J=4.2 \mathrm{~Hz}$, $2 \mathrm{H}), 3.77(\mathrm{q}, J=6.6 \mathrm{~Hz}, 2 \mathrm{H}), 3.38(\mathrm{t}, J=7.0 \mathrm{~Hz}, 2 \mathrm{H}), 1.51(\mathrm{~s}, 9 \mathrm{H}), 1.50(\mathrm{~s}, 9 \mathrm{H}) \cdot{ }^{13} \mathrm{C} \mathrm{NMR}$ (101 MHz, $\left.\mathrm{CDCl}_{3}\right): \delta 156.3,155.6,152.6,147.3,143.1,131.5,130.1,129.7,129.6,91.5$, 90.6, 83.8, 79.6, 69.0, 57.8, 50.9, 38.5, 28.4, 28.0, 23.6. IR ( $\left.\mathrm{cm}^{-1}\right): 1721,1638,1612,1569$, $1250,1225,1150,1130,1028,616$. HRMS (ESI+): $\mathrm{m} / z$ [M] ${ }^{+}$calcd for $\mathrm{C}_{30} \mathrm{H}_{38} \mathrm{~N}_{6} \mathrm{O}_{5} \mathrm{I}_{3}$ : 943.0038; found: 943.0028 .

\section{(S)-4-\{3-[N,N'-di(tert-Butoxycarbonyl)guanidyl]propyl\}-3-[2-(2,4,6-triiodophenoxy)-} ethyl]-1-\{N-[1-phenyl-2-(methoxycarbonyl)ethyl]carbamoylmethyl\}-5-iodo-1 H-1,2,3triazolium trifluoromethanesulfonate (2I)

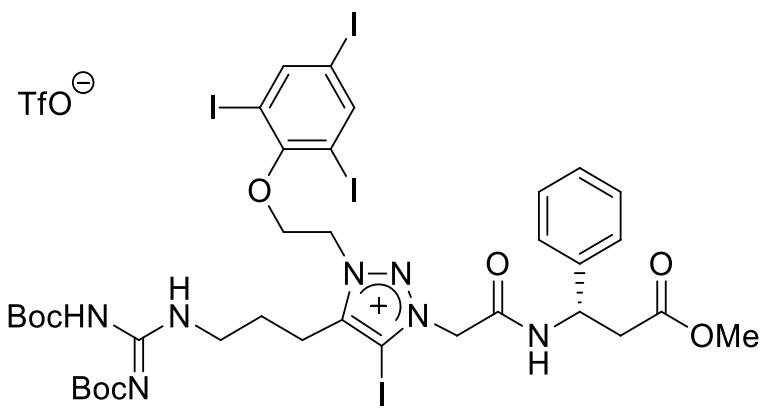

The general procedure was followed starting from (S)-4-\{3-[N,N'-di(tertbutoxycarbonyl)guanidyl]propyl\}-5-iodo1-\{N-[1-phenyl-2-(methoxycarbonyl)ethyl]carbamoylmethyl\}-1H-1,2,3-triazole $1 \mathrm{~g}(0.03 \mathrm{mmol}, 24 \mathrm{mg})$ and $2-(2,4,6-$ triiodophenoxy)ethyl trifluoromethanesulfonate $(0.04 \mathrm{mmol}, 25 \mathrm{mg})$. Yield: $32 \mathrm{mg}(77 \%)$. White solid $\left(\mathrm{mp}=108-110{ }^{\circ} \mathrm{C}\right) \cdot[\alpha]_{\mathrm{D}}{ }^{20}$ $=-28.35^{\circ}$ (c. $\left.0.96, \mathrm{CH}_{2} \mathrm{Cl}_{2}\right) .{ }^{1} \mathrm{H}$ NMR $\left(400 \mathrm{MHz}, \mathrm{CDCl}_{3}\right): \delta 11.48(\mathrm{~s}, 1 \mathrm{H}), 8.88(\mathrm{~d}, J=8.3$ $\mathrm{Hz}, 1 \mathrm{H}), 8.47(\mathrm{~s}, 1 \mathrm{H}), 8.00(\mathrm{~s}, 2 \mathrm{H}), 7.50-7.25(\mathrm{~m}, 5 \mathrm{H}), 5.58(\mathrm{~s}, 2 \mathrm{H}), 5.39$ (q, $J=7.6 \mathrm{~Hz}$, $1 \mathrm{H}), 5.17-5.02(\mathrm{~m}, 2 \mathrm{H}), 4.45-4.28(\mathrm{~m}, 2 \mathrm{H}), 3.65(\mathrm{~s}, 3 \mathrm{H}), 3.60-3.46(\mathrm{~m}, 2 \mathrm{H}), 3.16-2.80(\mathrm{~m}$, $4 \mathrm{H}), 2.17-2.00(\mathrm{~m}, 2 \mathrm{H}), 1.51(\mathrm{~s}, 18 \mathrm{H}) .{ }^{13} \mathrm{C} \mathrm{NMR}\left(101 \mathrm{MHz}, \mathrm{CDCl}_{3}\right): \delta 170.9,163.3,161.2$, 156.5, 155.4, 153.1, 148.9, 147.2, 140.3, 128.7, 127.7, 127.0, 92.0, 90.9, 83.5, 79.5, 69.0, 56.3, 51.9, 51.8, 51.1, 40.2, 39.7, 28.3, 28.1, 27.2, 22.3. IR ( $\left.\mathrm{cm}^{-1}\right): 1638,1276,1245,1155$, 1028, 637. HRMS (ESI+): $\mathrm{m} / z$ [M] ${ }^{+}$calcd for $\mathrm{C}_{36} \mathrm{H}_{46} \mathrm{~N}_{7} \mathrm{O}_{8} \mathrm{I}_{4}: 1211.9587$; found: 1211.9615 . 
4-\{1-[(R)- $\alpha$-Methylbenzyl]-1H-1,2,3-triazol-4-yl\}-1-[(R)- $\alpha$-methylbenzyl]-3-[2-(2,4,6triiodophenoxy)ethyl]-1H-1,2,3-triazolium trifluoromethanesulfonate (2m)

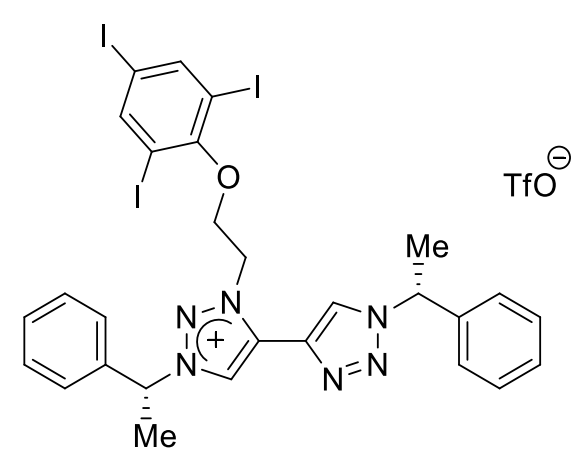

The general procedure was followed starting from 1,1'-bis[(R)- $\alpha$-methylbenzyl]-4,4'-bis $(1 H-1,2,3$-triazole) $\mathbf{1 h} \quad(0.03 \mathrm{mmol}, \quad 12 \mathrm{mg})$ and $2-(2,4,6-$ triiodophenoxy)ethyl trifluoromethanesulfonate $(0.03$ mmol, $22 \mathrm{mg}$ ). Yield: $27 \mathrm{mg}(81 \%)$. White solid (mp $\left.=54-56{ }^{\circ} \mathrm{C}\right) \cdot[\alpha]_{\mathrm{D}}{ }^{20}=-30.16^{\circ}\left(\mathrm{c}=0.93, \mathrm{CH}_{2} \mathrm{Cl}_{2}\right) \cdot{ }^{1} \mathrm{H}$ NMR (400 MHz, $\left.\mathrm{CDCl}_{3}\right): \delta 9.71(\mathrm{~s}, 1 \mathrm{H}), 9.16(\mathrm{~s}, 1 \mathrm{H})$, $7.98(\mathrm{~s}, 2 \mathrm{H}), 7.71-7.56(\mathrm{~m}, 3 \mathrm{H}), 7.53-7.30(\mathrm{~m}, 7 \mathrm{H})$, $6.14(\mathrm{q}, J=7.2 \mathrm{~Hz}, 1 \mathrm{H}), 5.92(\mathrm{q}, J=7.2 \mathrm{~Hz}, 1 \mathrm{H}), 5.64-5.45(\mathrm{~m}, 2 \mathrm{H}), 4.55-4.37(\mathrm{~m}, 2 \mathrm{H})$, $2.21(\mathrm{~d}, J=7.1 \mathrm{~Hz}, 3 \mathrm{H}), 2.06(\mathrm{~d}, J=7.1 \mathrm{~Hz}, 3 \mathrm{H}) .{ }^{13} \mathrm{C} \mathrm{NMR}\left(101 \mathrm{MHz}, \mathrm{CDCl}_{3}\right): \delta 155.6$, 147.3, 138.7, 136.0, 135.2, 131.7, 130.2, 129.7, 129.2, 128.9, 127.6, 127.4, 126.7, 126.4, $120.6(\mathrm{q}, J=320.0 \mathrm{~Hz}), 91.5,90.5,68.3,65.9,61.6,53.5,20.9,20.1 . \mathrm{IR}\left(\mathrm{cm}^{-1}\right): 1456$, 1256, 1223, 1150, 1027, 698, 636. HRMS (ESI+): $\mathrm{m} / z[\mathrm{M}]^{+}$calcd for $\mathrm{C}_{28} \mathrm{H}_{26} \mathrm{~N}_{6} \mathrm{OI}_{3}$ : 842.9302; found: 842.9306 .

\subsection{Synthesis of 1,5-disubstituted-1H-1,2,3-triazoles}

General Procedure A: A solution of the corresponding 1,2,3-triazole $(1.00 \mathrm{mmol})$ and MeOTf $(1.10 \mathrm{mmol})$ in anhydrous $\mathrm{CH}_{2} \mathrm{Cl}_{2}(5 \mathrm{~mL})$ was stirred under nitrogen atmosphere at ambient temperature for 18 hours. The solvents were removed under reduced pressure. The crude was dissolved in $\mathrm{MeOH}(5 \mathrm{~mL})$ and $\mathrm{K}_{2} \mathrm{CO}_{3}(2.00 \mathrm{mmol})$ was added. The mixture was stirred at ambient temperature for 5 hours. The solvents were removed under reduced pressure and the crude product was solved in $\mathrm{CH}_{2} \mathrm{Cl}_{2}$ and filtered by PTFE.

General Procedure B: To a solution of the corresponding 1,2,3-triazole $(1.00 \mathrm{mmol})$ in $\mathrm{CH}_{2} \mathrm{Cl}_{2}(8.6 \mathrm{~mL})$ the selected alkyl trifluoromethanesulfonate $(1.20 \mathrm{mmol})$ was added and evaporated under reduced pressure. After 18 hours at $30{ }^{\circ} \mathrm{C}$ the product was dissolved in $\mathrm{MeOH}(5 \mathrm{~mL})$ and $\mathrm{K}_{2} \mathrm{CO}_{3}(2.00 \mathrm{mmol})$ was added. The mixture was stirred at ambient temperature for 5 hours. The solvents were removed under reduced pressure and purified by column chromatography (silica gel, Hex/EtOAc 1:1). 


\section{1-Methyl-5-phenyl-1H-1,2,3-triazole (6a)}

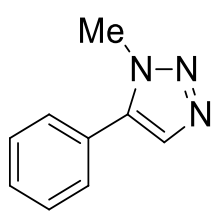

The general procedure A was followed starting from 4-phenyl-1(pivaloyloxymethyl)-1H-1,2,3-triazole 1i (0.60 mmol, $150 \mathrm{mg})$, MeOTf (0.64 mmol, $70 \mu \mathrm{L})$ and $\mathrm{CH}_{2} \mathrm{Cl}_{2}(2.50 \mathrm{~mL})$. The crude was dissolved in $\mathrm{MeOH}(2.50 \mathrm{~mL})$ and $\mathrm{K}_{2} \mathrm{CO}_{3}(1.20 \mathrm{mmol}, 166 \mathrm{mg})$ was added. Yield: $43 \mathrm{mg}$ (79 \%). Yellow oil. ${ }^{1} \mathrm{H}$ NMR (400 MHz, $\left.\mathrm{CDCl}_{3}\right): \delta 7.70(\mathrm{~s}, 1 \mathrm{H}), 7.47(\mathrm{~d}, J=7.4 \mathrm{~Hz}, 3 \mathrm{H})$, 7.42-7.35 (m, 2H), $4.04(\mathrm{~s}, 3 \mathrm{H}) .{ }^{13} \mathrm{C}$ NMR $\left(126 \mathrm{MHz}, \mathrm{CDCl}_{3}\right): \delta 138.4,133.3,129.5$, 129.1, 128.6, 127.0, 35.6. IR ( $\left.\mathrm{cm}^{-1}\right): 3423,2917,1749,1483,1244,1016,765,696$. HRMS (ESI+): $m / z[\mathrm{M}+\mathrm{H}]^{+}$calcd for $\mathrm{C}_{9} \mathrm{H}_{10} \mathrm{~N}_{3}: 160.0875$; found: 160.0869 .

\section{5-Butyl-1-methyl-1H-1,2,3-triazole (6b)}

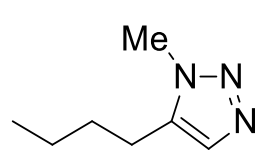

The general procedure A was followed starting from 4-butyl-1(pivaloyloxymethyl)-1H-1,2,3-triazole $\mathbf{1 j}$ (0.63 mmol, $150 \mathrm{mg}$ ), MeOTf (0.69 mmol, $80 \mu \mathrm{L}$ ) and $\mathrm{CH}_{2} \mathrm{Cl}_{2}(2.50 \mathrm{~mL})$. The crude was dissolved in $\mathrm{MeOH}(2.50 \mathrm{~mL})$ and $\mathrm{K}_{2} \mathrm{CO}_{3}(1.30 \mathrm{mmol}, 174 \mathrm{mg})$ was added. Yield: $73 \mathrm{mg}(83 \%)$. Yellow oil. ${ }^{~} \mathrm{H}$ NMR $\left(400 \mathrm{MHz}, \mathrm{CDCl}_{3}\right): \delta 7.34(\mathrm{~s}, 1 \mathrm{H}), 3.87(\mathrm{~s}, 3 \mathrm{H}), 2.54(\mathrm{t}, J=7.8 \mathrm{~Hz}$, $2 \mathrm{H}), 1.60-1.51(\mathrm{~m}, 2 \mathrm{H}), 1.33(\mathrm{q}, J=7.5 \mathrm{~Hz}, 2 \mathrm{H}), 0.87(\mathrm{t}, J=7.4 \mathrm{~Hz}, 3 \mathrm{H}) .{ }^{13} \mathrm{C}$ NMR $(101$ $\left.\mathrm{MHz}, \mathrm{CDCl}_{3}\right): \delta 137.4,131.9,34.1,29.9,22.7,22.1,13.6$. IR $\left(\mathrm{cm}^{-1}\right): 2956,2872,1749$, 1458, 1238, 1031. HRMS (ESI+): $\mathrm{m} / z[\mathrm{M}+\mathrm{H}]^{+}$calcd for $\mathrm{C}_{7} \mathrm{H}_{14} \mathrm{~N}_{3}$ : 140.1188; found: 140.1173 .

\section{5-Phenyl-1-(2-(2,4,6-triiodophenoxy)ethyl)-1H-1,2,3-triazole (6c)}

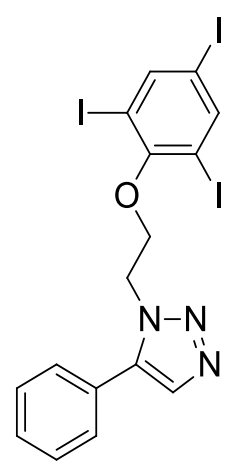

The general procedure $\mathrm{B}$ was followed starting from 4-phenyl-1(pivaloyloxymethyl)- $1 \mathrm{H}-1,2,3$-triazole $\mathbf{1 i}(0.24 \mathrm{mmol}, 60 \mathrm{mg})$ and 2-(2,4,6triiodophenoxy)ethyl trifluoromethanesulfonate $(0.29 \mathrm{mmol}, 187 \mathrm{mg})$. The crude was dissolved in $\mathrm{MeOH}(2.50 \mathrm{~mL})$ and $\mathrm{K}_{2} \mathrm{CO}_{3}(0.50 \mathrm{mmol}, 66 \mathrm{mg})$ was added. The product was purified by column chromatography (silica gel, $\mathrm{CH}_{2} \mathrm{Cl}_{2} / \mathrm{MeOH}$ 95:5). Yield: $105 \mathrm{mg}(68 \%)$. White solid ( $\mathrm{mp}=138-144$ $\left.{ }^{\circ} \mathrm{C}\right) .{ }^{1} \mathrm{H}$ NMR $\left(400 \mathrm{MHz}, \mathrm{CDCl}_{3}\right): \delta 7.99(\mathrm{~s}, 1 \mathrm{H}), 7.73(\mathrm{~s}, 2 \mathrm{H}), 7.60-7.53(\mathrm{~m}$, $2 \mathrm{H}), 7.48(\mathrm{~d}, J=5.7 \mathrm{~Hz}, 3 \mathrm{H}), 4.79(\mathrm{t}, J=5.4 \mathrm{~Hz}, 2 \mathrm{H}), 4.41(\mathrm{t}, J=5.4 \mathrm{~Hz}$,

2H). ${ }^{13} \mathrm{C}$ NMR (101 MHz, $\left.\mathrm{CDCl}_{3}\right): \delta 156.8,147.4,139.3,133.1,129.7,129.6,129.1$, 127.0, 91.8, 90.1, 70.9, 47.5. IR $\left(\mathrm{cm}^{-1}\right): 1421,1234,1024,768,697$. HRMS (ESI+): $\mathrm{m} / \mathrm{z}$ $[\mathrm{M}+\mathrm{H}]^{+}$calcd for $\mathrm{C}_{16} \mathrm{H}_{13} \mathrm{~N}_{3} \mathrm{OI}$ : 643.8193 ; found: 643.8207 . 


\section{1-Phenethyl-5-phenyl-1H-1,2,3-triazole (6d)}

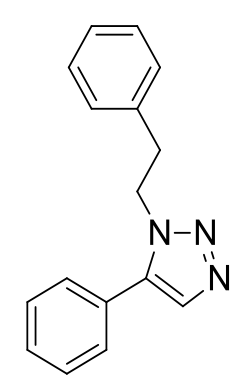

The general procedure $\mathrm{B}$ was followed starting from 4-phenyl-1(pivaloyloxymethyl)-1 $\mathrm{H}$-1,2,3-triazole $\quad \mathbf{1 i}(0.12 \mathrm{mmol}, 30 \mathrm{mg})$ and phenethyl trifluoromethanesulfonate $(0.14 \mathrm{mmol}, 35 \mathrm{mg})$. The crude was dissolved in $\mathrm{MeOH}(2.50 \mathrm{~mL})$ and $\mathrm{K}_{2} \mathrm{CO}_{3}(0.25 \mathrm{mmol}, 33 \mathrm{mg})$ was added. The product was purified by column chromatography (silica gel, Hex/EtOAc 7:3). Yield: $22 \mathrm{mg}(74 \%)$. White solid $\left(\mathrm{mp}=97-112{ }^{\circ} \mathrm{C}\right) .{ }^{1} \mathrm{H}$ NMR (400 MHz, MeOH-d 4 ): $\delta 8.15(\mathrm{~s}, 1 \mathrm{H}), 7.83(\mathrm{~d}, J=7.6 \mathrm{~Hz}, 2 \mathrm{H}), 7.44$ (t, $J=7.5 \mathrm{~Hz}, 1 \mathrm{H}), 7.36(\mathrm{t}, J=7.5 \mathrm{~Hz}, 1 \mathrm{H}), 7.30-7.09(\mathrm{~m}, 6 \mathrm{H}), 3.74(\mathrm{t}, J=7.2 \mathrm{~Hz}, 2 \mathrm{H})$, $2.81(\mathrm{t}, J=7.2 \mathrm{~Hz}, 2 \mathrm{H}) .{ }^{13} \mathrm{C}$ NMR $\left(101 \mathrm{MHz}, \mathrm{MeOH}-\mathrm{d}_{4}\right): \delta 140.3,131.2,130.0,130.0$, 129.9, 129.5, 129.3, 127.1, 127.0, 64.3, 40.3. IR $\left(\mathrm{cm}^{-1}\right): 1450,960,768$, 690. HRMS (ESI+): $m / z[\mathrm{M}+\mathrm{H}]^{+}$calcd for $\mathrm{C}_{16} \mathrm{H}_{16} \mathrm{~N}_{3}: 250.1344$; found: 250.1327 .

\section{1-(3-Bromopropyl)-5-phenyl-1H-1,2,3-triazole (6e)}

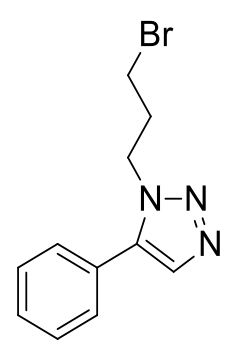

The general procedure $\mathrm{B}$ was followed starting from 4-phenyl-1(pivaloyloxymethyl)-1 $\mathrm{H}-1,2,3$-triazole $\mathbf{1 i}(0.24 \mathrm{mmol}, 60 \mathrm{mg})$ and 3 bromopropyl trifluoromethanesulfonate $(0.28 \mathrm{mmol}, 76 \mathrm{mg})$. The crude was dissolved in $\mathrm{MeOH}(2.50 \mathrm{~mL})$ and $\mathrm{K}_{2} \mathrm{CO}_{3}(0.50 \mathrm{mmol}, 66 \mathrm{mg})$ was added. The product was purified by column chromatography (silica gel, Hex/EtOAc 7:3). Yield: $33 \mathrm{mg}$ (52 \%). Yellow oil. ${ }^{1} \mathrm{H}$ NMR (400 MHz, $\left.\mathrm{CDCl}_{3}\right): \delta 7.70(\mathrm{~s}, 1 \mathrm{H}), 7.55-7.46(\mathrm{~m}, 3 \mathrm{H}), 7.44-7.35(\mathrm{~m}, 2 \mathrm{H}), 4.50(\mathrm{t}, J=6.8 \mathrm{~Hz}, 2 \mathrm{H})$, $3.36(\mathrm{t}, J=6.3 \mathrm{~Hz}, 2 \mathrm{H}), 2.53-2.31(\mathrm{~m}, 2 \mathrm{H}) .{ }^{13} \mathrm{C} \mathrm{NMR}\left(101 \mathrm{MHz}, \mathrm{CDCl}_{3}\right): \delta 138.2,133.2$, 129.7, 129.3, 128.9, 126.9, 46.5, 32.7, 29.6. IR $\left(\mathrm{cm}^{-1}\right): 2960,1482,1455,1274,1245$, 1014, 959, 833, 763, 697. HRMS (ESI+): $m / z \mathrm{M}^{+}$calcd for $\mathrm{C}_{11} \mathrm{H}_{12} \mathrm{~N}_{3}$ : 186.1031; found: 186.1018 .

\section{1-(3-Cyanopropyl)-5-phenyl-1H-1,2,3-triazole (6f)}

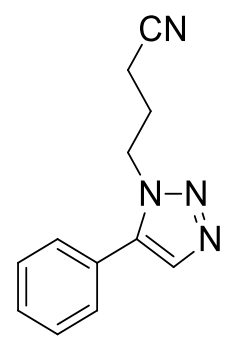

The general procedure $\mathrm{B}$ was followed starting from 4-phenyl-1(pivaloyloxymethyl)-1 $\mathrm{H}-1,2,3$-triazole $\mathbf{1 i}(0.50 \mathrm{mmol}, 130 \mathrm{mg})$ and 3 bromopropyl trifluoromethanesulfonate $(0.60 \mathrm{mmol}, 163 \mathrm{mg})$. The crude was dissolved in $\mathrm{MeOH}(2.50 \mathrm{~mL})$ and $\mathrm{K}_{2} \mathrm{CO}_{3}(1.00 \mathrm{mmol}, 138 \mathrm{mg})$ was added. The product was purified by column chromatography (silica gel, Hex/EtOAc 7:3). After that, the product, 1-(3-bromopropyl)-5-phenyl-1H1,2,3-triazole $(0.24 \mathrm{mmol}, 64 \mathrm{mg})$ was dissolved in $\mathrm{EtOH} / \mathrm{H}_{2} \mathrm{O}$ 7:3 $(3 \mathrm{~mL})$ and $\mathrm{KCN}(0.65$ 
mmol, $43 \mathrm{mg}$ ) was added. The mixture was stirred for $45 \mathrm{~min}$ at $90^{\circ} \mathrm{C}$, and after it, stirred room temperature overnight. The solvent was evaporated under reduced pressure, and the aqueous phase was extracted with EtOAc $(10 \mathrm{~mL} \times 3)$. The organic layer was dried over $\mathrm{MgSO}_{4}$ and the solvent was evaporated under reduced pressure. The product was purified by column chromatography (silica gel, Hex/EtOAc 1:1). Yield: $36 \mathrm{mg}$ (70 \%). White solid $\left(\mathrm{mp}=55-57{ }^{\circ} \mathrm{C}\right) .{ }^{1} \mathrm{H}$ NMR $\left(400 \mathrm{MHz}, \mathrm{CDCl}_{3}\right): \delta 7.70(\mathrm{~s}, 1 \mathrm{H}), 7.61-7.46(\mathrm{~m}, 3 \mathrm{H}), 7.45-$ $7.31(\mathrm{~m}, 2 \mathrm{H}), 4.47(\mathrm{t}, J=6.6 \mathrm{~Hz}, 2 \mathrm{H}), 2.41(\mathrm{t}, J=7.1 \mathrm{~Hz}, 2 \mathrm{H}), 2.22(\mathrm{p}, J=6.9 \mathrm{~Hz}, 2 \mathrm{H})$. ${ }^{13} \mathrm{C}$ NMR $\left(101 \mathrm{MHz}, \mathrm{CDCl}_{3}\right): \delta 138.2,133.4,129.9,129.4,128.8,126.6,118.3,46.4,25.8$, 14.8. IR $\left(\mathrm{cm}^{-1}\right): 1738,1483,1233,964,840,769,696,537$. HRMS (ESI+): $\mathrm{m} / z \mathrm{M}^{+}$calcd for $\mathrm{C}_{12} \mathrm{H}_{13} \mathrm{~N}_{4}$ : 213.1140; found: 213.1134 .

\section{1-(Pent-4-yn-1-yl)-5-phenyl-1H-1,2,3-triazole (6g)}

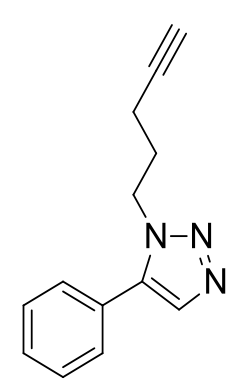

The general procedure $\mathrm{B}$ was followed starting from 4-phenyl-1(pivaloyloxymethyl)-1H-1,2,3-triazole $1 \mathbf{i}(0.24 \mathrm{mmol}, 60 \mathrm{mg})$ and pent-4yn-1-yl trifluoromethanesulfonate $(0.28 \mathrm{mmol}, 61 \mathrm{mg})$. The crude was dissolved in $\mathrm{MeOH}(2.50 \mathrm{~mL})$ and $\mathrm{K}_{2} \mathrm{CO}_{3}(0.50 \mathrm{mmol}, 66 \mathrm{mg})$ was added. The product was purified by column chromatography (silica gel, Hex/EtOAc 7:3). Yield: $14 \mathrm{mg}(27 \%)$. Colorless oil. ${ }^{1} \mathrm{H}$ NMR (400 MHz, $\left.\mathrm{CDCl}_{3}\right): \delta 7.70(\mathrm{~s}, 1 \mathrm{H}), 7.54-7.37(\mathrm{~m}, 5 \mathrm{H}), 4.47(\mathrm{t}, J=7.0 \mathrm{~Hz}, 2 \mathrm{H}), 2.21$ $(\mathrm{td}, J=6.9,2.4 \mathrm{~Hz}, 2 \mathrm{H}), 2.09(\mathrm{p}, J=6.9 \mathrm{~Hz}, 2 \mathrm{H}), 1.89(\mathrm{t}, J=2.6 \mathrm{~Hz}, 1 \mathrm{H}) .{ }^{13} \mathrm{C} \mathrm{NMR}(101$ $\left.\mathrm{MHz}, \mathrm{CDCl}_{3}\right): \delta 133.2,129.6,129.2,129.0,127.2,82.3,69.8,61.2,47.0,28.8,15.9$. IR $\left(\mathrm{cm}^{-1}\right): 3293,2948,1283,1413,1240,1208,965,764,697,637$. HRMS (ESI+): $\mathrm{m} / \mathrm{z} \mathrm{M}^{+}$ calcd for $\mathrm{C}_{13} \mathrm{H}_{14} \mathrm{~N}_{3}$ : 212.1188; found: 212.1195.

\section{1-(3-Azidopropyl)-5-phenyl-1H-1,2,3-triazole (6h)}

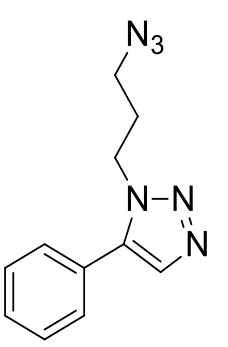

The general procedure B was followed starting from 4-phenyl-1(pivaloyloxymethyl)-1H-1,2,3-triazole $1 \mathbf{1 i}(0.12 \mathrm{mmol}, 30 \mathrm{mg})$ and 3 azidopropyl trifluoromethanesulfonate $(0.14 \mathrm{mmol}, 32.6 \mathrm{mg})$. The crude was dissolved in $\mathrm{MeOH}(2.50 \mathrm{~mL})$ and $\mathrm{K}_{2} \mathrm{CO}_{3}(0.24 \mathrm{mmol}, 33 \mathrm{mg})$ was added. The product was purified by column chromatography (silica gel, Hex/EtOAc 1:1). Yield: $11 \mathrm{mg}$ (40\%). Colorless oil. ${ }^{1} \mathrm{H}$ NMR (400 MHz, $\left.\mathrm{CDCl}_{3}\right): \delta 7.72(\mathrm{~s}, 1 \mathrm{H}), 7.57-7.46(\mathrm{~m}, 3 \mathrm{H}), 7.45-7.34(\mathrm{~m}, 2 \mathrm{H}), 4.44(\mathrm{t}, J=6.9 \mathrm{~Hz}, 2 \mathrm{H})$, $3.34(\mathrm{t}, J=6.4 \mathrm{~Hz}, 2 \mathrm{H}), 2.12(\mathrm{p}, J=6.7 \mathrm{~Hz}, 2 \mathrm{H}) .{ }^{13} \mathrm{C} \mathrm{NMR}\left(101 \mathrm{MHz}, \mathrm{CDCl}_{3}\right): \delta 138.3$, 133.2, 129.8, 129.4, 128.9, 126.9, 48.3 , 45.5, 29.3. IR ( $\left.\mathrm{cm}^{-1}\right): 2094,1482,1455,1238,964$, 764, 697. HRMS (ESI+): $m / z[\mathrm{M}+\mathrm{H}]^{+}$calcd for $\mathrm{C}_{11} \mathrm{H}_{13} \mathrm{~N}_{6}: 229.1202$; found: 229.1187. 
Hex/EtOAc 1:1). Yield: $198 \mathrm{mg}$ (60 \%). Colorless oil. ${ }^{1} \mathrm{H} \mathrm{NMR}\left(400 \mathrm{MHz}, \mathrm{CDCl}_{3}\right): \delta 7.48$ $(\mathrm{s}, 1 \mathrm{H}), 4.36(\mathrm{t}, J=5.9 \mathrm{~Hz}, 2 \mathrm{H}), 3.86(\mathrm{t}, J=5.9 \mathrm{~Hz}, 2 \mathrm{H}), 2.71-2.62(\mathrm{~m}, 2 \mathrm{H}), 1.67(\mathrm{p}, J=$ $7.5 \mathrm{~Hz}, 2 \mathrm{H}), 1.43(\mathrm{dq}, J=14.6,7.4 \mathrm{~Hz}, 2 \mathrm{H}), 0.97(\mathrm{t}, J=7.3 \mathrm{~Hz}, 3 \mathrm{H}) .{ }^{13} \mathrm{C} \mathrm{NMR}(101 \mathrm{MHz}$, $\left.\mathrm{CDCl}_{3}\right): \delta 137.7,131.2,50.1,46.0,29.5,22.1,21.6,13.1 . \mathrm{IR}\left(\mathrm{cm}^{-1}\right): 2958,2933,2097$, 1455, 1286, 1237, 827. HRMS (ESI+): $m / z \mathrm{M}^{+}$calcd for $\mathrm{C}_{8} \mathrm{H}_{15} \mathrm{~N}_{6}$ : 195.1358; found: 195.1360 .

\subsection{Synthesis of 1,4,5-trisubstituted-1H-1,2,3-triazoles}

\section{1-Methyl-5-phenyl-1H-1,2,3-triazole-4- $d$ (7)}

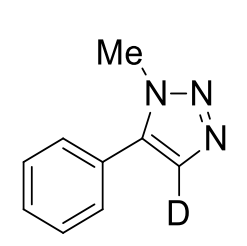

To a solution of 4-phenyl-1-(pivaloyloxymethyl)- $1 H$-1,2,3-triazole 1i $(0.18$ mmol, $48 \mathrm{mg})$ and $\operatorname{MeOTf}(0.20 \mathrm{mmol}, 22 \mu \mathrm{L})$ in $\mathrm{CDCl}_{3}(2.50 \mathrm{~mL})$ was stirred under nitrogen atmosphere at ambient temperature for 18 hours. The volatiles were removed under reduced pressure. The crude was dissolved in $\mathrm{MeOH}-\mathrm{d}_{4}(2.50 \mathrm{~mL})$ and $\mathrm{K}_{2} \mathrm{CO}_{3}(0.15 \mathrm{mmol}, 21 \mathrm{mg})$ was added. The mixture was stirred at ambient temperature for 5 hours. The solvents were removed under reduced pressure and the crude product was solved in $\mathrm{CH}_{2} \mathrm{Cl}_{2}$ and filtered by PTFE. Yield: $9 \mathrm{mg}(75 \%)$. Colorless oil. ${ }^{1} \mathrm{H} \mathrm{NMR}\left(400 \mathrm{MHz}, \mathrm{CDCl}_{3}\right): \delta 7.54(\mathrm{~m}, 5 \mathrm{H}), 4.10(\mathrm{~s}, 3 \mathrm{H}) .{ }^{13} \mathrm{C}$ NMR (101 MHz, $\left.\mathrm{CDCl}_{3}\right): \delta 139.9,130.7,130.2,129.8,127.9,127.0,36.1 . \mathrm{IR}\left(\mathrm{cm}^{-1}\right): 2933$, 1460, 1259, 1028, 772. HRMS (ESI+): $m / z[\mathrm{M}+\mathrm{H}]^{+}$calcd for $\mathrm{C}_{9} \mathrm{H}_{9} \mathrm{DN}_{3}: 161.0937$; found: 161.0929 .

\section{4-Iodo-1-methyl-5-phenyl-1H-1,2,3-triazole (8a)}

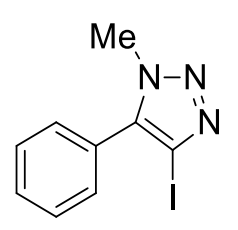

To a solution of 4-phenyl-1-(pivaloyloxymethyl)-1H-1,2,3-triazole 1i (0.40 mmol, $104 \mathrm{mg})$ in $\mathrm{CH}_{2} \mathrm{Cl}_{2}(2 \mathrm{~mL})$, MeOTf $(0.44 \mathrm{mmol}, 50 \mu \mathrm{L})$ was added and the mixture was stirred for 5 hours at ambient temperature. Then, triflate exchange was carried out by using an anion exchange resin ( $\mathrm{I}^{-}$form) 
following a procedure reported in the literature, ${ }^{19}$ and using $\mathrm{MeCN}$ as solvent. After that, a mixture of 3-methyl-4-phenyl-1-(pivaloyloxymethyl)-1H-1,2,3-triazolium iodide, $\operatorname{Ag}_{2} \mathrm{O}$ (0.24 mmol, $48 \mathrm{mg}$ ) and cyanogen halide (0.80 mmol, $122 \mathrm{mg})$ in $\mathrm{CH}_{2} \mathrm{Cl}_{2} / \mathrm{MeCN}^{1: 1}$ (3 $\mathrm{mL}$ ) was stirred at ambient temperature for 6 hours. The mixture was filtered through a celite/silice 1:1 pad which was successively washed with $\mathrm{CH}_{2} \mathrm{Cl}_{2}$ and $\mathrm{MeCN}$. Finally, the product was dissolved in $\mathrm{MeOH}(2 \mathrm{~mL})$ and $\mathrm{K}_{2} \mathrm{CO}_{3}(0.80 \mathrm{mmol}, 110 \mathrm{mg})$ was added. The mixture was stirred at ambient temperature for 5 hours. The solvents were removed under reduced pressure and the product was purified by column chromatography (silica gel, Hex/EtOAc 1:1). Yield: $114 \mathrm{mg}(>99 \%)$. Yellowish solid $\left(\mathrm{mp}=102-124{ }^{\circ} \mathrm{C}\right) .{ }^{1} \mathrm{H}$ NMR $\left(400 \mathrm{MHz}, \mathrm{CDCl}_{3}\right): \delta$ 7.54-7.47 (m, 3H), 7.40-7.33 (m, 2H), $3.99(\mathrm{~s}, 3 \mathrm{H}) .{ }^{13} \mathrm{C}$ NMR (101 $\left.\mathrm{MHz}, \mathrm{CDCl}_{3}\right): \delta 140.2,130.1,129.6,129.1,126.0,89.7,36.3 . \mathrm{IR}\left(\mathrm{cm}^{-1}\right): 2949,1481,1449$, 1258, 1031, 980, 772, 697, 542. HRMS (ESI+): $m / z,[\mathrm{M}+\mathrm{H}]^{+}$calcd for $\mathrm{C}_{9} \mathrm{H}_{9} \mathrm{~N}_{3} \mathrm{I}: 285.9841$; found: 285.9833 .

\section{5-Butyl-4-iodo-1-(2-(2,4,6-triiodophenoxy)ethyl)-1H-1,2,3-triazole (8b)}

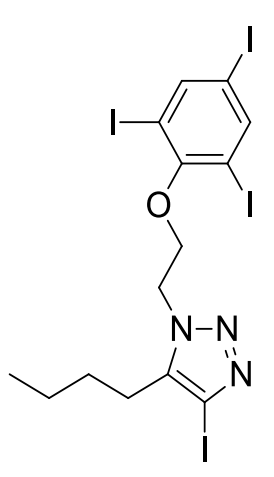

To a solution of 4-butyl-1-(pivaloyloxymethyl)-1H-1,2,3-triazole $\mathbf{1 j}$ $(0.40 \mathrm{mmol}, 96 \mathrm{mg})$ in $\mathrm{CH}_{2} \mathrm{Cl}_{2}(2 \mathrm{~mL})$, MeOTf $(0.44 \mathrm{mmol}, 50 \mu \mathrm{L})$ was added and the mixture was stirred at ambient temperature for 5 hours. Then, triflate exchange was carried out by using an anion exchange resin ( $\mathrm{I}^{-}$form) following a procedure reported in the literature, ${ }^{19}$ and using $\mathrm{MeCN}$ as solvent. After that, a mixture of 4-butyl-3-methyl-1(pivaloyloxymethyl)-1H-1,2,3-triazolium iodide, $\mathrm{Ag}_{2} \mathrm{O}$ (0.24 mmol, 48 $\mathrm{mg}$ ) and cyanogen halide $(0.80 \mathrm{mmol}, 122 \mathrm{mg})$ in $\mathrm{CH}_{2} \mathrm{Cl}_{2} / \mathrm{MeCN}$ 1:1 (3

$\mathrm{mL}$ ) was stirred at ambient temperature for 6 hours. The mixture was filtered through a celite/silice 1:1 pad which was successively washed with $\mathrm{CH}_{2} \mathrm{Cl}_{2}$ and $\mathrm{MeCN}$. Finally, the product was dissolved in $\mathrm{MeOH}(2 \mathrm{~mL})$ and $\mathrm{K}_{2} \mathrm{CO}_{3}(0.80 \mathrm{mmol}, 110 \mathrm{mg})$ was added. The mixture was stirred at ambient temperature for 5 hours. The solvents were removed under reduced pressure and the product was purified by column chromatography (silica gel, Hex/EtOAc 1:1). Yield: $192 \mathrm{mg}(64 \%)$. White solid $\left(\mathrm{mp}=102-124{ }^{\circ} \mathrm{C}\right) .{ }^{1} \mathrm{H}$ NMR $(400$ $\left.\mathrm{MHz}, \mathrm{CDCl}_{3}\right): \delta 8.00(\mathrm{~s}, 2 \mathrm{H}), 4.76(\mathrm{t}, J=5.2 \mathrm{~Hz}, 2 \mathrm{H}), 4.32(\mathrm{t}, J=5.1 \mathrm{~Hz}, 2 \mathrm{H}), 2.85-2.76$ $(\mathrm{m}, 2 \mathrm{H}), 1.61(\mathrm{p}, J=7.7 \mathrm{~Hz}, 2 \mathrm{H}), 1.40(\mathrm{~h}, J=7.3 \mathrm{~Hz}, 2 \mathrm{H}), 0.94(\mathrm{t}, J=7.2 \mathrm{~Hz}, 3 \mathrm{H}) .{ }^{13} \mathrm{C}$ NMR (101 MHz, $\left.\mathrm{CDCl}_{3}\right): \delta 156.5,147.4,141.4,91.8,90.3,89.4,71.0,48.4,30.6,23.4$,

19 Dinarés, I.; Mesquida, N.; Ibáñez, A.; Alcalde, E. ARKIVOC 2014, ii, 85. 
22.5. IR ( $\left.\mathrm{cm}^{-1}\right): 2923,1524,1462,1423,1377,1220,1028,1005,852,651$. HRMS (ESI+): $\mathrm{m} / z[\mathrm{M}+\mathrm{H}]^{+}$calcd for $\mathrm{C}_{14} \mathrm{H}_{16} \mathrm{~N}_{3} \mathrm{OI}_{4}: 749.7472$; found: 749.7486 .

\subsection{Synthesis of bistriazoles and tetrahydrohexaazaindacenium salts}

General Procedure: To a solution of the corresponding azide $(1.00 \mathrm{mmol})$ and alkyne $(1.10 \mathrm{mmol})$ in $\mathrm{THF} / \mathrm{t} \mathrm{BuOH} / \mathrm{H}_{2} \mathrm{O} \quad 1: 1: 1(5 \mathrm{~mL})$, sodium ascorbate $(0.40 \mathrm{mmol})$ and $\mathrm{CuSO}_{4} \cdot 5 \mathrm{H}_{2} \mathrm{O}(0.20 \mathrm{mmol})$ were added. The reaction mixture was stirred at ambient temperature for 18 hours. The organic solvents were evaporated under reduced pressure and the aqueous residue was extracted with EtOAc/ $/ \mathrm{NH}_{3} 20 \%\left(\mathrm{H}_{2} \mathrm{O}\right)$. The organic layer was separated, dried over $\mathrm{MgSO}_{4}$ and the solvents were evaporated under reduced pressure. The crude product was purified by column chromatography (silica gel, Hex/EtOAc).

\section{5-Phenyl-1-[3-(4-phenyl-1H-1,2,3-triazol-1-yl)propyl]-1H-1,2,3-triazole (9a)}

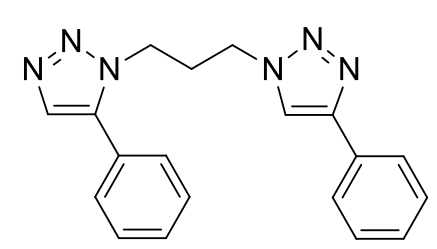

The general procedure was followed starting from 1-(3azidopropyl)-5-phenyl-1 $\mathrm{H}$-1,2,3-triazole $\mathbf{6 i}(0.07 \mathrm{mmol}, 16.5$ $\mathrm{mg})$, ethynylbenzene $(0.09 \mathrm{mmol}, 10 \mu \mathrm{L}), \mathrm{CuSO}_{4} \cdot 5 \mathrm{H}_{2} \mathrm{O}(14$ $\mu \mathrm{mol}, 4.20 \mathrm{mg})$ and sodium ascorbate $(28 \mu \mathrm{mol}, 5.60 \mathrm{mg})$ in $\mathrm{THF} / \mathrm{BuOH} / \mathrm{H}_{2} \mathrm{O}$ 1:1:1 (1 mL). Yield: $22 \mathrm{mg}(>99 \%)$. White solid (mp $\left.=111-115^{\circ} \mathrm{C}\right) .{ }^{1} \mathrm{H}$ NMR $\left(400 \mathrm{MHz}, \mathrm{CDCl}_{3}\right)$ : $\delta$ 7.84-7.74 (m, 3H), $7.71(\mathrm{~s}, 1 \mathrm{H})$, 7.49-7.24 (m, 8H), $4.44(\mathrm{t}, J=6.2 \mathrm{~Hz}, 2 \mathrm{H}), 4.41-4.33(\mathrm{~m}, 2 \mathrm{H}), 2.59-2.42(\mathrm{~m}, 2 \mathrm{H}) .{ }^{13} \mathrm{C}$ NMR (101 MHz, $\left.\mathrm{CDCl}_{3}\right): \delta 173.0,147.8,130.4,129.9,129.4,128.9,128.7,128.3,126.5$, 125.8, 120.6, 47.1, 44.9, 30.3, 22.7. IR ( $\left(\mathrm{cm}^{-1}\right): 764,696$. HRMS (ESI+): $\mathrm{m} / \mathrm{z}[\mathrm{M}+\mathrm{H}]^{+}$calcd for $\mathrm{C}_{19} \mathrm{H}_{19} \mathrm{~N}_{6}: 331.1671$; found: 331.1664 .

\section{5-Butyl-1-(2-(4-hydroxymethyl-1H-1,2,3-triazol-1-yl)ethyl)-1H-1,2,3-triazole (9b)}

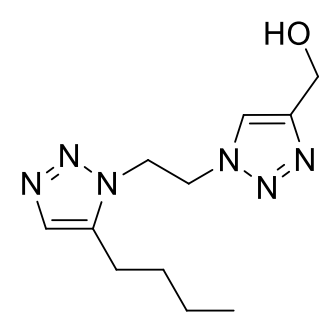

The general procedure was followed starting from 1-(2-azidoethyl)-5butyl-1H-1,2,3-triazole $\mathbf{6 j}$ (1.87 mmol, $364 \mathrm{mg}$ ), prop-2-yn-1-ol (2.10 $\mathrm{mmol}, 0.12 \mathrm{~mL}), \mathrm{CuSO}_{4} \cdot 5 \mathrm{H}_{2} \mathrm{O}(0.40 \mathrm{mmol}, 110 \mathrm{mg})$ and sodium ascorbate $(0.70 \mathrm{mmol}, 148 \mathrm{mg})$ in $\mathrm{THF} / \mathrm{BuOH} / \mathrm{H}_{2} \mathrm{O}$ 1:1:1 $(14 \mathrm{~mL})$. Yield: $415 \mathrm{mg}(89 \%)$. Yellowish oil. ${ }^{1} \mathrm{H}$ NMR $\left(400 \mathrm{MHz}, \mathrm{CDCl}_{3}\right): \delta$ $7.35(\mathrm{~s}, 1 \mathrm{H}), 7.26(\mathrm{~s}, 1 \mathrm{H}), 5.03(\mathrm{~s}, 1 \mathrm{H}), 4.85(\mathrm{t}, J=5.9 \mathrm{~Hz}, 2 \mathrm{H}), 4.65$ (t, $J=5.9 \mathrm{~Hz}, 2 \mathrm{H}), 4.56(\mathrm{~s}, 2 \mathrm{H}), 2.21(\mathrm{t}, J=7.8 \mathrm{~Hz}, 2 \mathrm{H}), 1.37(\mathrm{p}, J=7.9,7.3 \mathrm{~Hz}, 2 \mathrm{H})$, 
1.26-1.13 (m, 2H), $\left.0.78(\mathrm{t}, J=7.4 \mathrm{~Hz}, 3 \mathrm{H}) .{ }^{13} \mathrm{C} \mathrm{NMR} \mathrm{(101} \mathrm{MHz,} \mathrm{CDCl}_{3}\right): \delta 148.1,138.3$, 131.5, 123.1, 55.4, 49.0, 46.8, 29.5, 21.8, 21.8, 13.3. IR $\left(\mathrm{cm}^{-1}\right): 3346,2931,1667,1455$, 1236, 1039, 823, 765. HRMS (ESI+): $m / z,[\mathrm{M}+\mathrm{H}]^{+}$calcd for $\mathrm{C}_{11} \mathrm{H}_{19} \mathrm{~N}_{6} \mathrm{O}: 251.1620$; found: 251.1623 .

\section{1,1'-[Di(pivaloyloxymethyl)]-4,4'-bis(1H-1,2,3-triazole) (11)}

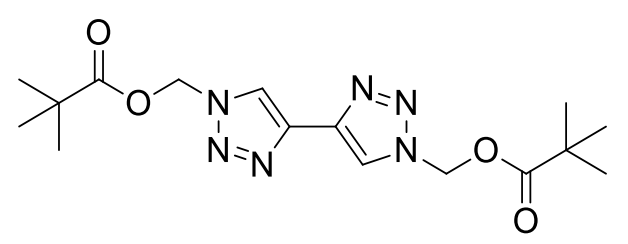

$\mathrm{KOH}$ (4.3 g, $65 \mathrm{mmol})$, water (10 mL), and DMSO $(2.5 \mathrm{~mL})$ were heated to $75^{\circ} \mathrm{C}$ for $30 \mathrm{~min}$ in a threenecked flask equipped with a condenser and a dropping funnel. The top of the condenser was connected, via a tube, to a trap containing dry THF,

which was cooled to $-20{ }^{\circ} \mathrm{C}$. 1,4-dichloro-2-butyne $(1.60 \mathrm{~mL}, 16 \mathrm{mmol})$ was added dropwise over a period of $30 \mathrm{~min}$, while the temperature was maintained at $75^{\circ} \mathrm{C}$. A stream of argon was passed through the apparatus, which forced the butadiyne into the cold THF trap. The tube was extracted, and azidomethyl pivalate 10 (1.02 g, $6.6 \mathrm{mmol}$ ), sodium acetate $(1.6 \mathrm{~g}, 19.8 \mathrm{mmol})$ and $\mathrm{CuOAc}(240 \mathrm{mg}, 1.32 \mathrm{mmol})$ were added at ambient temperature for 4 hours. The solution was filtered through a celite pad, and the solvents were evaporated under reduced pressure. The crude product was purified by column chromatography (silica gel, Hex/EtOAc 7:3). Yield: $1.06 \mathrm{~g}(85 \%)$. White solid $(\mathrm{mp}=198-$ $\left.203{ }^{\circ} \mathrm{C}\right) .{ }^{1} \mathrm{H}$ NMR $\left(400 \mathrm{MHz}, \mathrm{CDCl}_{3}\right): \delta 8.01(\mathrm{~s}, 2 \mathrm{H}), 6.26(\mathrm{~s}, 4 \mathrm{H}), 1.20(\mathrm{~s}, 18 \mathrm{H}) .{ }^{13} \mathrm{C} \mathrm{NMR}$ $\left(126 \mathrm{MHz} \mathrm{CDCl}_{3}\right): \delta 177.6,140.3,122.2,69.9,38.9,26.9 . \mathrm{IR}\left(\mathrm{cm}^{-1}\right): 2971,1731,1279$, 1144, 1057, 828. HRMS (ESI+): $m / z$. $[\mathrm{M}+\mathrm{H}]^{+}$calcd for $\mathrm{C}_{16} \mathrm{H}_{25} \mathrm{~N}_{6} \mathrm{O}_{4}$ : 365.1937; found: 365.1950 .

\section{1,1'-Dimethyl-5,5'-bis(1,2,3-triazole) (12)}

The general procedure was followed starting from $1,1^{\prime}-$
[di(pivaloyloxymethyl)]-4,4'-bis $(1 \mathrm{H}-1,2,3$-triazole $) \mathbf{1 1}(0.22 \mathrm{mmol}, 80 \mathrm{mg})$,
MeOTf $(1.2 \mathrm{mmol}, 160 \mu \mathrm{L})$, and $\mathrm{CH}_{2} \mathrm{Cl}_{2}(1.2 \mathrm{~mL})$. The reaction mixture and the crude was solved in $\mathrm{MeOH}(2.50 \mathrm{~mL})$ and $\mathrm{K}_{2} \mathrm{CO}_{3}(0.88 \mathrm{mmol}, 121 \mathrm{mg})$ was added. The reaction was stirred at ambient temperature for 3 hours. Yield: $22 \mathrm{mg}(61 \%)$. White solid $\left(\mathrm{mp}=125-142{ }^{\circ} \mathrm{C}\right) .{ }^{1} \mathrm{H}$ NMR $\left(400 \mathrm{MHz}, \mathrm{CDCl}_{3}\right): \delta 7.89(\mathrm{~s}, 2 \mathrm{H}), 4.07(\mathrm{~s}, 6 \mathrm{H}) \cdot{ }^{13} \mathrm{C}$ NMR (101 MHz, $\left.\mathrm{CDCl}_{3}\right): \delta 134.5,124.7,35.8 . \mathrm{IR}\left(\mathrm{cm}^{-1}\right): 3133,1664,1452,1252,1149$, 1024, 954, 858, 679. HRMS (ESI+): $m / z,[\mathrm{M}+\mathrm{H}]^{+}$calcd for $\mathrm{C}_{6} \mathrm{H}_{9} \mathrm{~N}_{6}$ : 165.0889; found: 165.0892 . 


\section{1-Benzyl-1'-methyl-4,5'-bis(1H-1,2,3-triazole) (13a)}

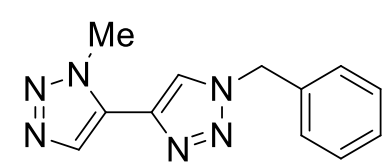

A solution of 4-ethynyl-1-(pivaloxymethyl)-1 $\mathrm{H}$-1,2,3-triazole $\mathbf{1 k}$ $(0.24 \mathrm{mmol}, 50 \mathrm{mg})$ and MeOTf $(0.36 \mathrm{mmol}, 41 \mu \mathrm{L})$ in $\mathrm{CH}_{2} \mathrm{Cl}_{2}$ $(2.50 \mathrm{~mL})$ was stirred under nitrogen atmosphere at room temperature for 1 hour. After that, the solvent was removed under reduced pressure. Then, benzylazide $(0.24 \mathrm{mmol}, 32 \mathrm{mg}), \mathrm{CuSO}_{4} \cdot 5 \mathrm{H}_{2} \mathrm{O}(0.05 \mathrm{mmol}, 12 \mathrm{mg})$, sodium ascorbate $(0.10 \mathrm{mmol}, 19 \mathrm{mg})$ and $\mathrm{NaOAc}(0.24 \mathrm{mmol}, 20 \mathrm{mg})$ in $\mathrm{THF} / \mathrm{BuOH} / \mathrm{H}_{2} \mathrm{O}$ 1:1:1 $(2 \mathrm{~mL})$ were added and the mixture was stirred at ambient temperature for 18 hours. Finally, the volatiles were removed under reduced pressure, the crude product was dissolved in $\mathrm{MeOH}$ $(2.50 \mathrm{~mL})$ and $\mathrm{K}_{2} \mathrm{CO}_{3}(0.48 \mathrm{mmol}, 66 \mathrm{mg})$ was added. The resulting mixture was stirred at ambient temperature for 3 hours. The solvents were removed under reduced pressure and the crude product was purified by column chromatography (silica gel, Hex/EtOAc). Yield: $43.5 \mathrm{mg}(75 \%)$. Yellow solid $\left(\mathrm{mp}=103-115^{\circ} \mathrm{C}\right) .{ }^{1} \mathrm{H}$ NMR $\left(400 \mathrm{MHz}, \mathrm{CDCl}_{3}\right): \delta 7.79(\mathrm{~s}$, $1 \mathrm{H}), 7.75(\mathrm{~s}, 1 \mathrm{H}), 7.46-7.23(\mathrm{~m}, 5 \mathrm{H}), 5.58(\mathrm{~s}, 2 \mathrm{H}), 4.30(\mathrm{~s}, 3 \mathrm{H}) .{ }^{13} \mathrm{C}$ NMR $(101 \mathrm{MHz}$, $\left.\mathrm{CDCl}_{3}\right): \delta 136.1,134.0,132.5,129.3,129.2,128.5,128.3,122.2,54.5,37.0 . \mathrm{IR}\left(\mathrm{cm}^{-1}\right)$ : 3079, 1426, 1243, 1025, 827, 731. HRMS (ESI+): $\mathrm{m} / \mathrm{z}[\mathrm{M}+\mathrm{H}]^{+}$calcd for $\mathrm{C}_{12} \mathrm{H}_{13} \mathrm{~N}_{6}$ : 241.1202; found: 241.1203 .

\section{1-Methyl-1'-phenyl-5,4'-bis(1,2,3-triazole) (13b)}

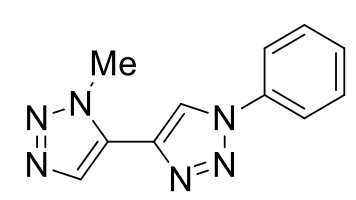

A solution of 4-ethynyl-1-(pivaloxymethyl)-1H-1,2,3-triazole $\mathbf{1 k}$ $(0.24 \mathrm{mmol}, 50 \mathrm{mg})$ and MeOTf $(0.36 \mathrm{mmol}, 41 \mu \mathrm{L})$ in $\mathrm{CH}_{2} \mathrm{Cl}_{2}$ $(2.50 \mathrm{~mL})$ was stirred under nitrogen atmosphere at ambient temperature for 1 hour. After that, the solvent was removed under reduced pressure. Then, phenylazide $(0.24 \mathrm{mmol}, 29 \mathrm{mg}), \mathrm{CuSO}_{4} \cdot 5 \mathrm{H}_{2} \mathrm{O}(0.05 \mathrm{mmol}, 12$ $\mathrm{mg})$, sodium ascorbate $(0.10 \mathrm{mmol}, 19 \mathrm{mg})$ and $\mathrm{NaOAc}(0.24 \mathrm{mmol}, 20 \mathrm{mg})$ in $\mathrm{THF} / \mathrm{BuOH} / \mathrm{H}_{2} \mathrm{O}$ 1:1:1 $(2 \mathrm{~mL})$ were added and the mixture was stirred at ambient temperature for 18 hours. Finally, the volatiles were removed under reduced pressure, the crude product was dissolved in $\mathrm{MeOH}(2.50 \mathrm{~mL})$ and $\mathrm{K}_{2} \mathrm{CO}_{3}(0.48 \mathrm{mmol}, 66 \mathrm{mg})$ was added. The resulting mixture was stirred at ambient temperature for 3 hours. The solvents were removed under reduced pressure and the crude product was purified by column chromatography (silica gel, Hex/EtOAc). Yield: $54 \mathrm{mg}(>99 \%)$. White solid (mp = 119$\left.130{ }^{\circ} \mathrm{C}\right) .{ }^{1} \mathrm{H}$ NMR $\left(400 \mathrm{MHz}, \mathrm{CDCl}_{3}\right): \delta 8.38(\mathrm{~s}, 1 \mathrm{H}), 7.90(\mathrm{~s}, 1 \mathrm{H}), 7.76(\mathrm{~d}, J=7.8 \mathrm{~Hz}$, $2 \mathrm{H}), 7.52(\mathrm{t}, J=7.7 \mathrm{~Hz}, 2 \mathrm{H}), 7.46(\mathrm{~d}, J=7.2 \mathrm{~Hz}, 1 \mathrm{H}), 4.35(\mathrm{~s}, 3 \mathrm{H}) .{ }^{13} \mathrm{C} \mathrm{NMR}(101 \mathrm{MHz}$, $\left.\mathrm{CDCl}_{3}\right): \delta 136.1,136.0,129.5,128.9,120.7,120.2,116.4,36.8$. IR $\left(\mathrm{cm}^{-1}\right): 3119,2962$, 
2920, 1599, 1509, 1458, 1418, 1248, 1176, 1030 749. HRMS (ESI+): $m / z[\mathrm{M}+\mathrm{H}]^{+}$calcd for $\mathrm{C}_{11} \mathrm{H}_{11} \mathrm{~N}_{6}: 227.1045$; found: 227.1040 .

\section{1-Benzyl-1'-(2-(2,4,6-triiiodophenoxy)ethyl)-5,4'-bis(1,2,3-triazole) (13c)}

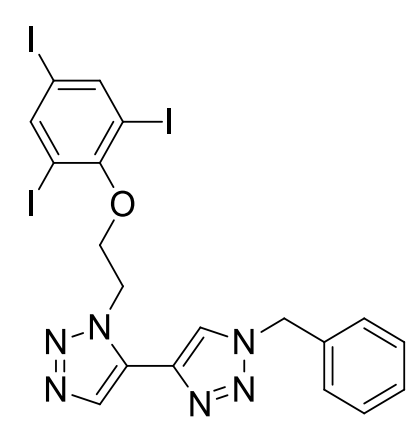

To a solution of 4-ethynyl-1-(pivaloxymethyl)-1H-1,2,3-triazole $1 \mathbf{k}(0.29 \mathrm{mmol}, 60 \mathrm{mg})$ in $\mathrm{CH}_{2} \mathrm{Cl}_{2}(0.50 \mathrm{~mL})$, 2-(2,4,6triiodophenoxy)ethyl trifluoromethanesulfonate ( $0.35 \mathrm{mmol}, 225$ $\mathrm{mg}$ ) was added and evaporated under reduced pressure. After 18 hours at $30{ }^{\circ} \mathrm{C}$, benzylazide $(0.29 \mathrm{mmol}, 39 \mathrm{mg}), \mathrm{CuSO}_{4} \cdot 5 \mathrm{H}_{2} \mathrm{O}$ $(0.06 \mathrm{mmol}, 14 \mathrm{mg})$, sodium ascorbate $(0.12 \mathrm{mmol}, 23 \mathrm{mg})$ and $\mathrm{NaOAc}(0.29 \mathrm{mmol}, 24 \mathrm{mg})$ in $\mathrm{THF} /{ }^{t} \mathrm{BuOH} / \mathrm{H}_{2} \mathrm{O}$ 1:1:1 (2 mL) were added and the mixture was stirred at ambient temperature for 18 hours. Finally, the volatiles were removed under reduced pressure, the crude product was dissolved in $\mathrm{MeOH}$ $(3.00 \mathrm{~mL})$ and $\mathrm{K}_{2} \mathrm{CO}_{3}(0.6 \mathrm{mmol}, 80 \mathrm{mg})$ was added. The resulting mixture was stirred at ambient temperature for 3 hours. The solvents were removed under reduced pressure and the crude product was purified by column chromatography (silica gel, Hex/EtOAc). Yield: $166 \mathrm{mg}(79 \%)$. White solid $\left(\mathrm{mp}=63-76{ }^{\circ} \mathrm{C}\right)$. ${ }^{1} \mathrm{H} \mathrm{NMR}\left(400 \mathrm{MHz}, \mathrm{CDCl}_{3}\right): \delta$ 7.96-7.90 $(\mathrm{m}, 3 \mathrm{H}), 7.85(\mathrm{~s}, 1 \mathrm{H}), 7.35-7.23(\mathrm{~m}, 5 \mathrm{H}), 5.57(\mathrm{~s}, 2 \mathrm{H}), 5.13(\mathrm{t}, J=5.2 \mathrm{~Hz}, 2 \mathrm{H}), 4.36(\mathrm{t}, J=$ $5.1 \mathrm{~Hz}, 2 \mathrm{H}) .{ }^{13} \mathrm{C} \mathrm{NMR}\left(101 \mathrm{MHz}, \mathrm{CDCl}_{3}\right): \delta$ 156.6, 147.3, 135.9, 134.1, 132.8, 129.3, 129.1, 128.2, 122.9, 91.8, 90.0, 71.1, 54.5, 48.9. IR $\left(\mathrm{cm}^{-1}\right): 3123,1425,1229,1045,717$, 695. HRMS (ESI+): $m / z[\mathrm{M}+\mathrm{H}]^{+}$calcd for $\mathrm{C}_{19} \mathrm{H}_{16} \mathrm{~N}_{6} \mathrm{OI}_{3}: 724.8520$; found: 724.8529 .

\section{[3-(3-Azidopropyl)-1-benzyl-4-ethynyl-1H-1,2,3-triazolium trifluoromethanesulfonate} (15)

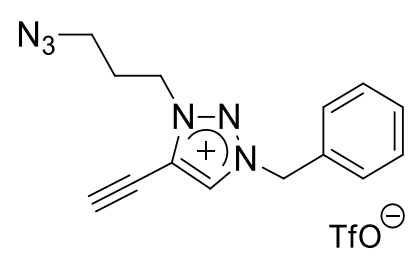

A solution of 1-benzyl-4-ethynyl-1H-1,2,3-triazole 1d $(0.30$ $\mathrm{mmol}, 55 \mathrm{mg})$ and 3-azidopropyl-1-triflate $14(0.33 \mathrm{mmol}, 93$ $\mathrm{mg})$ in $\mathrm{MeCN}(0.6 \mathrm{~mL})$ was stirred for 18 hours at $30^{\circ} \mathrm{C}$. Then, the solvent was removed under reduced pressure and the crude product was purified by column chromatography (silica gel,

$\left.\mathrm{CH}_{2} \mathrm{Cl}_{2} / \mathrm{MeOH}\right)$. Yield: $75 \mathrm{mg}(60 \%)$. Colorless oil. ${ }^{1} \mathrm{H} \mathrm{NMR}\left(400 \mathrm{MHz}, \mathrm{CDCl}_{3}\right): \delta 8.84$ $(\mathrm{s}, 1 \mathrm{H}), 7.67-7.33(\mathrm{~m}, 5 \mathrm{H}), 5.85(\mathrm{~s}, 2 \mathrm{H}), 4.72(\mathrm{t}, J=6.9 \mathrm{~Hz}, 2 \mathrm{H}), 4.24(\mathrm{~s}, 1 \mathrm{H}), 3.51(\mathrm{t}, J=$ $6.1 \mathrm{~Hz}, 2 \mathrm{H}), 2.28(\mathrm{p}, J=6.6 \mathrm{~Hz}, 2 \mathrm{H}) .{ }^{13} \mathrm{C} \mathrm{NMR}\left(101 \mathrm{MHz}, \mathrm{CDCl}_{3}\right): \delta 133.3,131.0,130.4$, $130.0,129.8,126.1,120.8(\mathrm{q}, J=320.0 \mathrm{~Hz}), 95.1,64.3,58.6,50.6,48.0,28.2 . \mathrm{IR}\left(\mathrm{cm}^{-1}\right)$ : $3206,2101,1458,1254,1224,1153,1029,737,636$.$] .$ 


\section{2-Benzyl-4,5-dihydro-2,3,3a,5a,6,7-hexaaza-indacenium trifluoromethanesulfonate (17)}

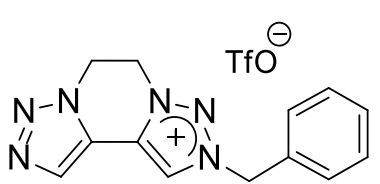

A solution of 1-benzyl-4-ethynyl-1H-1,2,3-triazole 1d (1.09 mmol, $200 \mathrm{mg}$ ) and 2-azidoethyl trifluoromethanesulfonate $\mathbf{1 6}$ $(1.20 \mathrm{mmol}, 263 \mathrm{mg})$ in $\mathrm{MeCN}(4 \mathrm{~mL})$ was stirred at $30{ }^{\circ} \mathrm{C}$ for 18 hours. Then, the solvent was removed under reduced pressure and the crude product was purified by column chromatography (silica gel, $\mathrm{CH}_{2} \mathrm{Cl}_{2} / \mathrm{MeOH}$ ). Yield: $320 \mathrm{mg}(73 \%)$. White solid $\left(\mathrm{mp}=106-108{ }^{\circ} \mathrm{C}\right) .{ }^{1} \mathrm{H} \mathrm{NMR}\left(400 \mathrm{MHz}, \mathrm{MeCN}-\mathrm{d}_{3}\right)$ : $\delta$ $8.83(\mathrm{~s}, 1 \mathrm{H}), 8.29(\mathrm{~s}, 1 \mathrm{H}), 7.71-7.38(\mathrm{~m}, 5 \mathrm{H}), 5.88(\mathrm{~s}, 2 \mathrm{H}), 5.20-4.97(\mathrm{~m}, 4 \mathrm{H}) .{ }^{13} \mathrm{C}$ NMR $\left(101 \mathrm{MHz}, \mathrm{MeCN}-\mathrm{d}_{3}\right): \delta$ 132.2, 131.3, 130.5, 129.6, 129.1, 129.0, 125.7, 121.4, 57.4, 47.4, 43.6. IR $\left(\mathrm{cm}^{-1}\right): 1458,1277,1223,1130,733,634$. HRMS (ESI+): $\mathrm{m} / \mathrm{z}[\mathrm{M}]^{+}$calcd for $\mathrm{C}_{13} \mathrm{H}_{13} \mathrm{~N}_{6}$ : 253.1202; found: 253.1203.

\section{2-Benzyl-3'-methyl-4,5-dihydro-2,3,3a,5a,6,7-hexaaza-indacenium ditrifluoromethanesulfonate (18)}

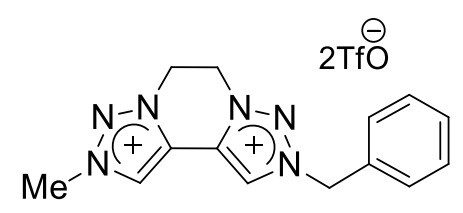

A solution of 2-benzyl-4,5-dihydro-2,3,3a,5a,6,7-hexaazaindacenium trifluoromethanesulfonate $17(0.08 \mathrm{mmol}, 34 \mathrm{mg})$ and MeOTf $(0.09 \mathrm{mmol}, 10 \mu \mathrm{L})$ in $\mathrm{CH}_{2} \mathrm{Cl}_{2}(0.5 \mathrm{~mL})$ was stirred at ambient temperature for 5 hours. Then, the solvent was removed under reduced pressure and the crude product was purified by column chromatography (silica gel, $\left.\mathrm{CH}_{2} \mathrm{Cl}_{2} / \mathrm{MeOH}\right)$. Yield: $40 \mathrm{mg}(84 \%)$. White solid ( $\mathrm{mp}=186$ $187^{\circ} \mathrm{C}$ ). ${ }^{1} \mathrm{H}$ NMR $\left(400 \mathrm{MHz}, \mathrm{MeCN}_{-} \mathrm{d}_{3}\right.$ ): $\delta 9.15$ (s, 1H), 9.06 (s, 1H), 7.66-7.46 (m, 5H), $5.94(\mathrm{~s}, 2 \mathrm{H}), 5.30(\mathrm{~s}, 4 \mathrm{H}), 4.43$ (s, 3H). ${ }^{13} \mathrm{C}$ NMR (101 MHz, MeCN-d $\left.{ }_{3}\right): \delta 130.9,129.7$, 129.7, 129.3, 129.0, 128.8, 126.8, 126.5, 120.4 (q, $J=320.0 \mathrm{~Hz}$ ), 57.9, 46.9, 46.7, 40.9. IR $\left(\mathrm{cm}^{-1}\right): 1459,1245,1225,1161,1028,709,637$. HRMS (ESI+): $\mathrm{m} / z[\mathrm{M}-\mathrm{H}]^{+}$calcd for $\mathrm{C}_{14} \mathrm{H}_{15} \mathrm{~N}_{6}$ : 267.1358; found: 267.1354. 


\section{2. ${ }^{1} \mathrm{H}-\mathrm{NMR}$ and ${ }^{13} \mathrm{C}-\mathrm{NMR}$ spectra of compounds $1 \mathrm{~b}, 1 \mathrm{f}-\mathrm{g}, 1 \mathrm{j}-\mathrm{k}, \mathbf{2 a - m}, \mathbf{6 - 1 3}, 15-18$.}

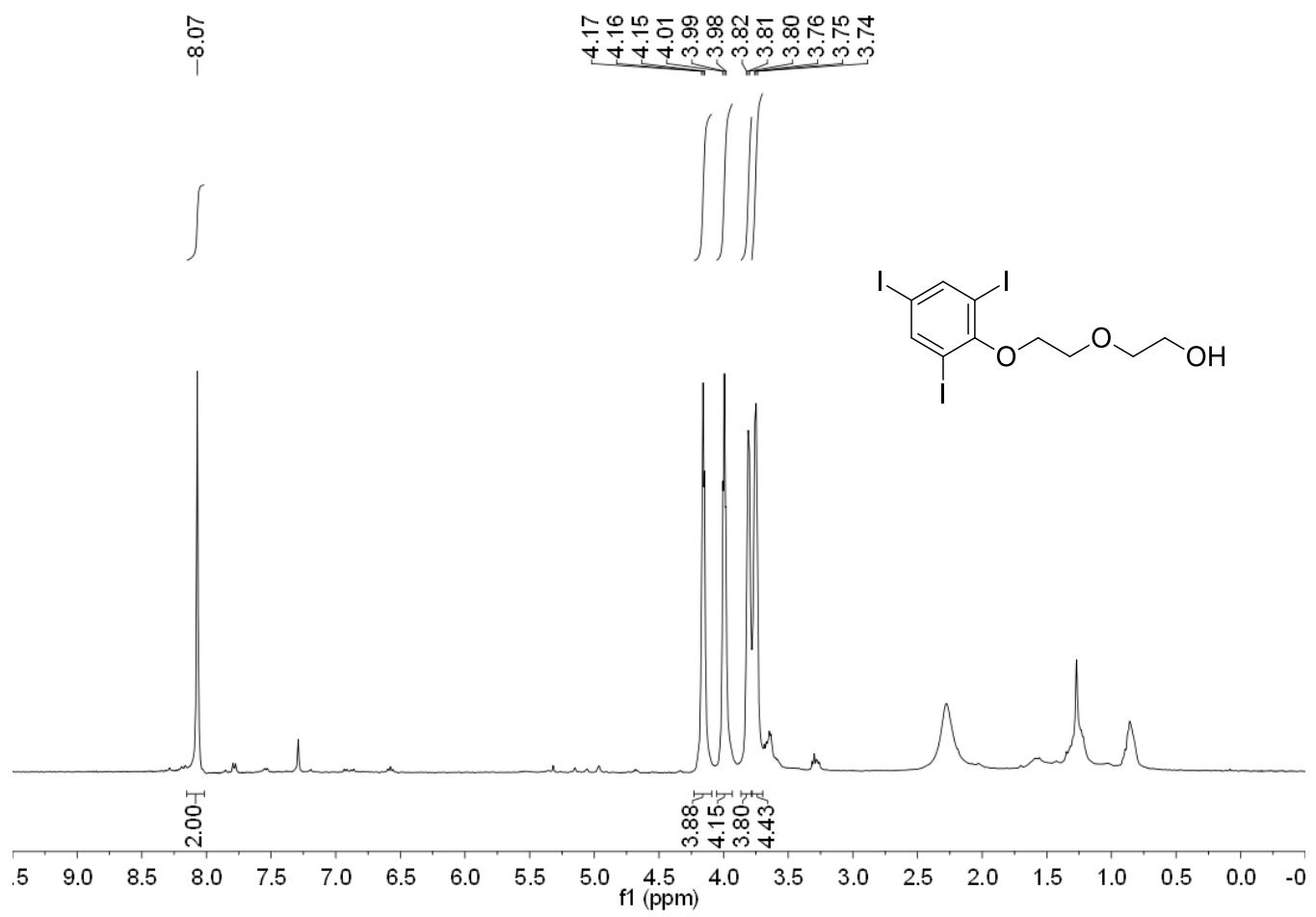

Spectrum 1. ${ }^{1} \mathrm{H}$ NMR $\left(400 \mathrm{MHz}, \mathrm{CDCl}_{3}\right)$ spectrum of 2-[2-(2,4,6-triiodophenoxy)ethoxy] ethanol.

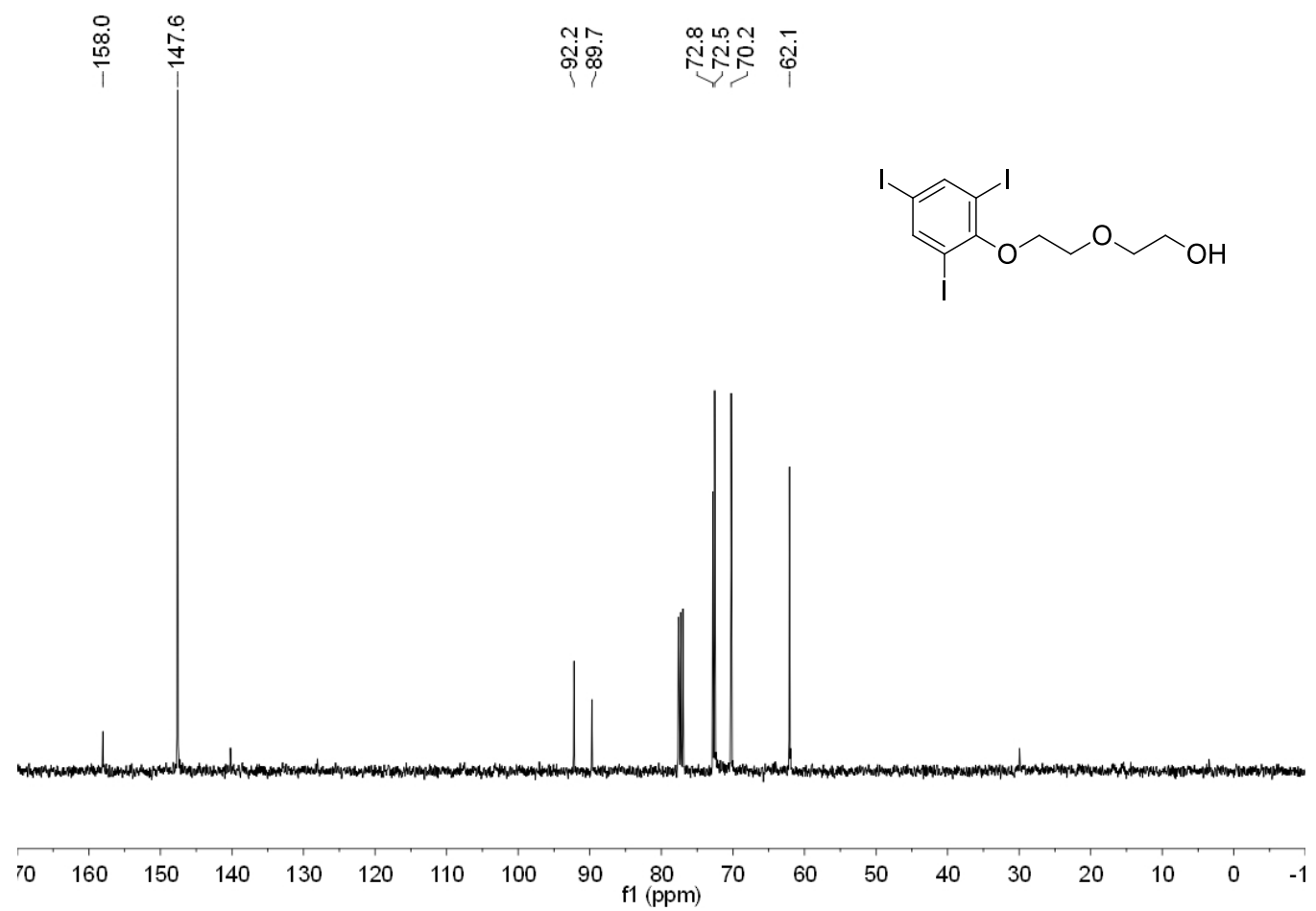

Spectrum 2. ${ }^{13} \mathrm{C}$ NMR $\left(400 \mathrm{MHz}, \mathrm{CDCl}_{3}\right)$ spectrum of 2-[2-(2,4,6-triiodophenoxy)ethoxy]ethanol. 

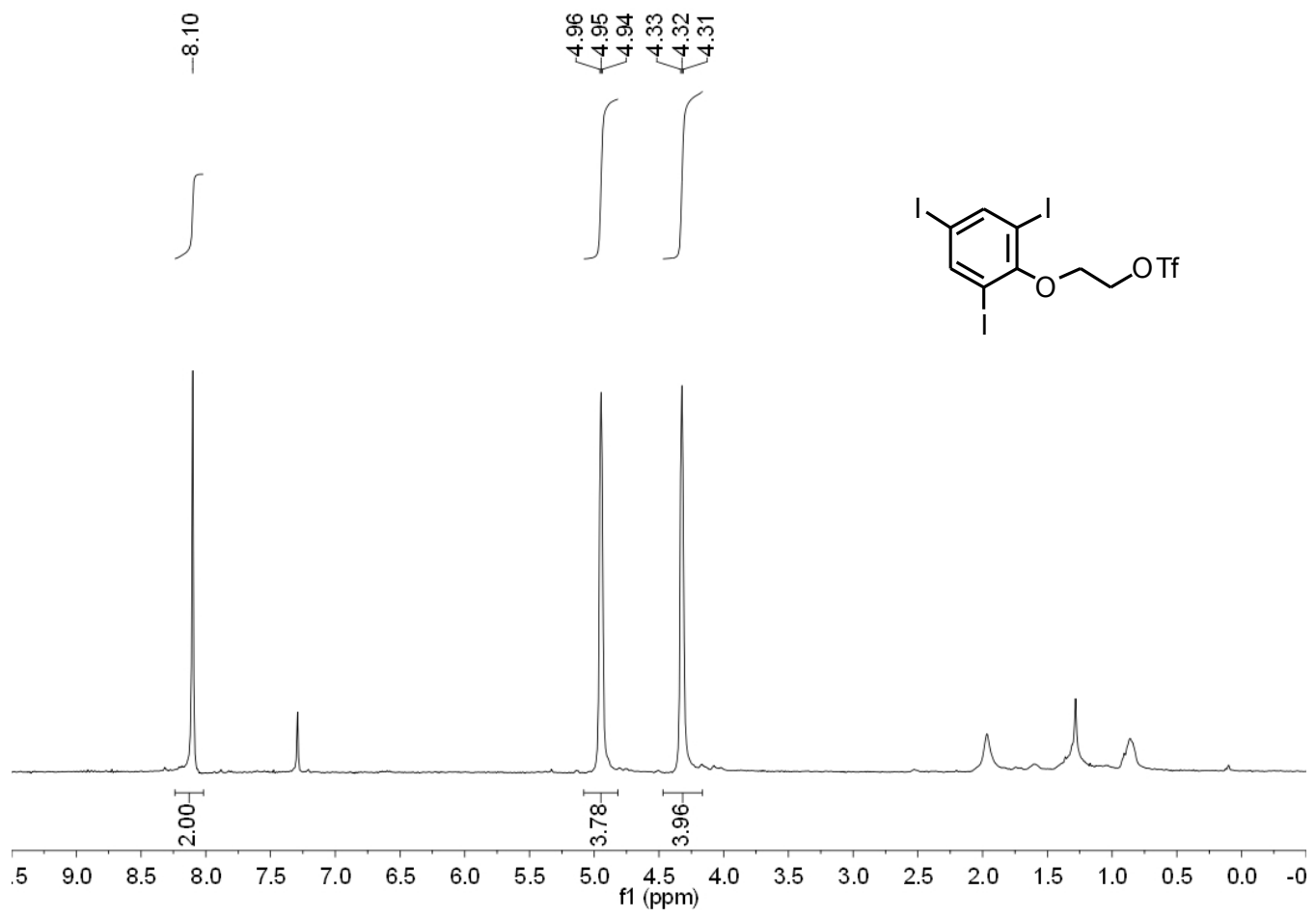

Spectrum 3. ${ }^{1} \mathrm{H}$ NMR (400 $\left.\mathrm{MHz}, \mathrm{CDCl}_{3}\right)$ spectrum of 2-(2,4,6-triiodophenoxy)ethyl trifluoromethanesulfonate.

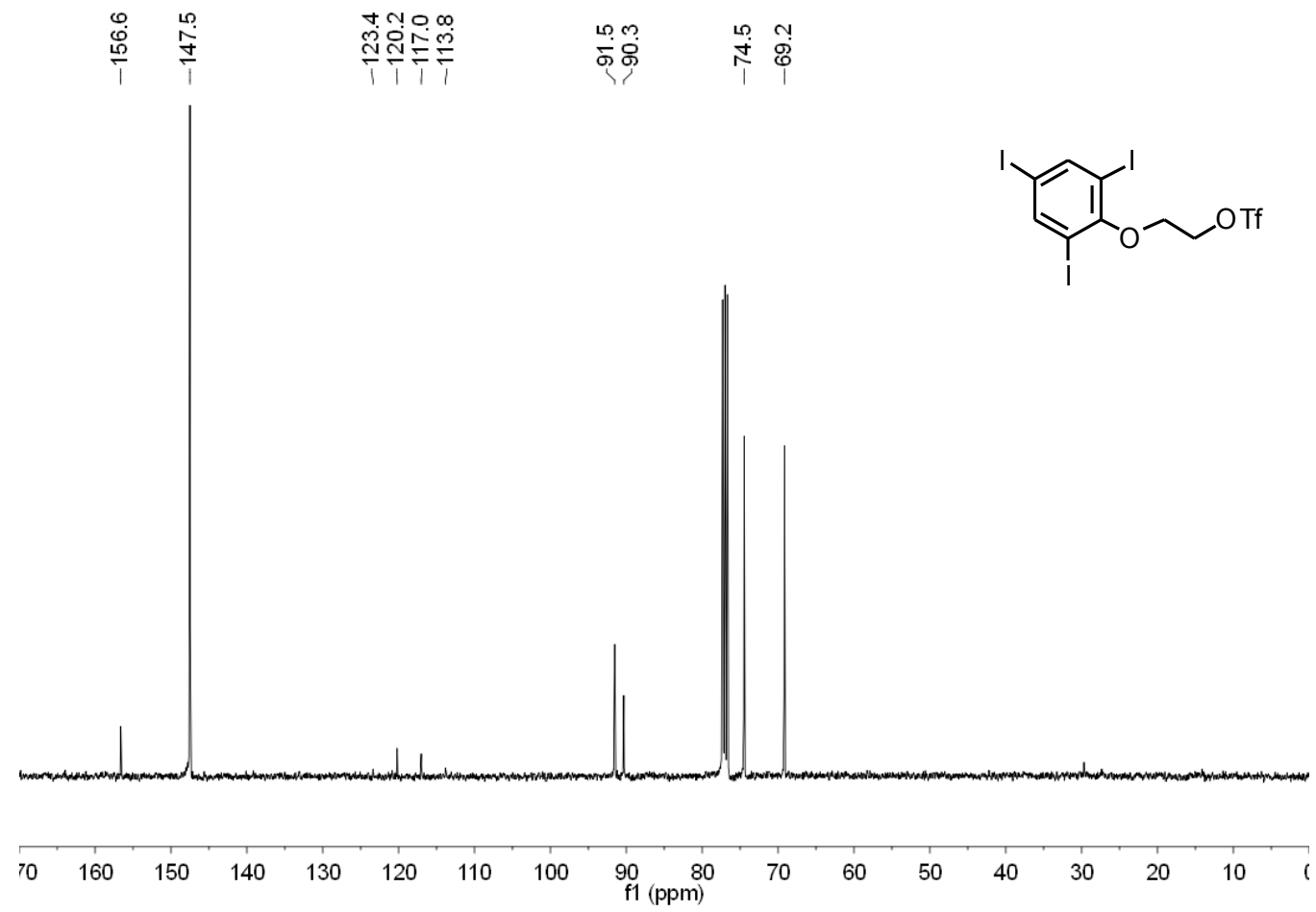

Spectrum 4. ${ }^{13} \mathrm{C}$ NMR (101 $\left.\mathrm{MHz}, \mathrm{CDCl}_{3}\right)$ spectrum of 2-(2,4,6-triiodophenoxy)ethyl trifluoromethanesulfonate. 


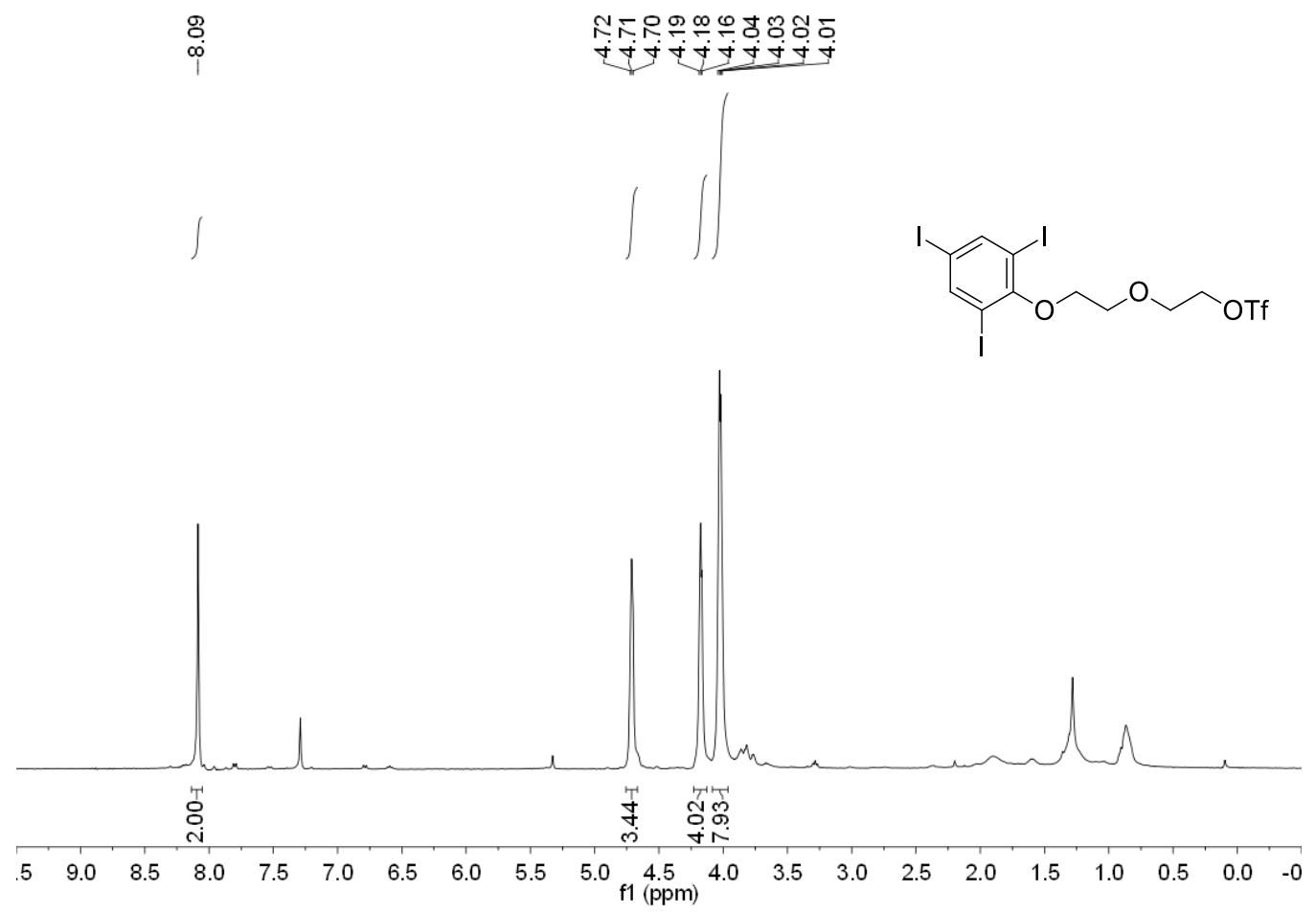

Spectrum 5. $\quad{ }^{1} \mathrm{H}$ NMR (400 MHz, $\left.\mathrm{CDCl}_{3}\right)$ spectrum of 2-[2-(2,4,6-triiodophenoxy)ethoxy]ethyl trifluoromethanesulfonate.
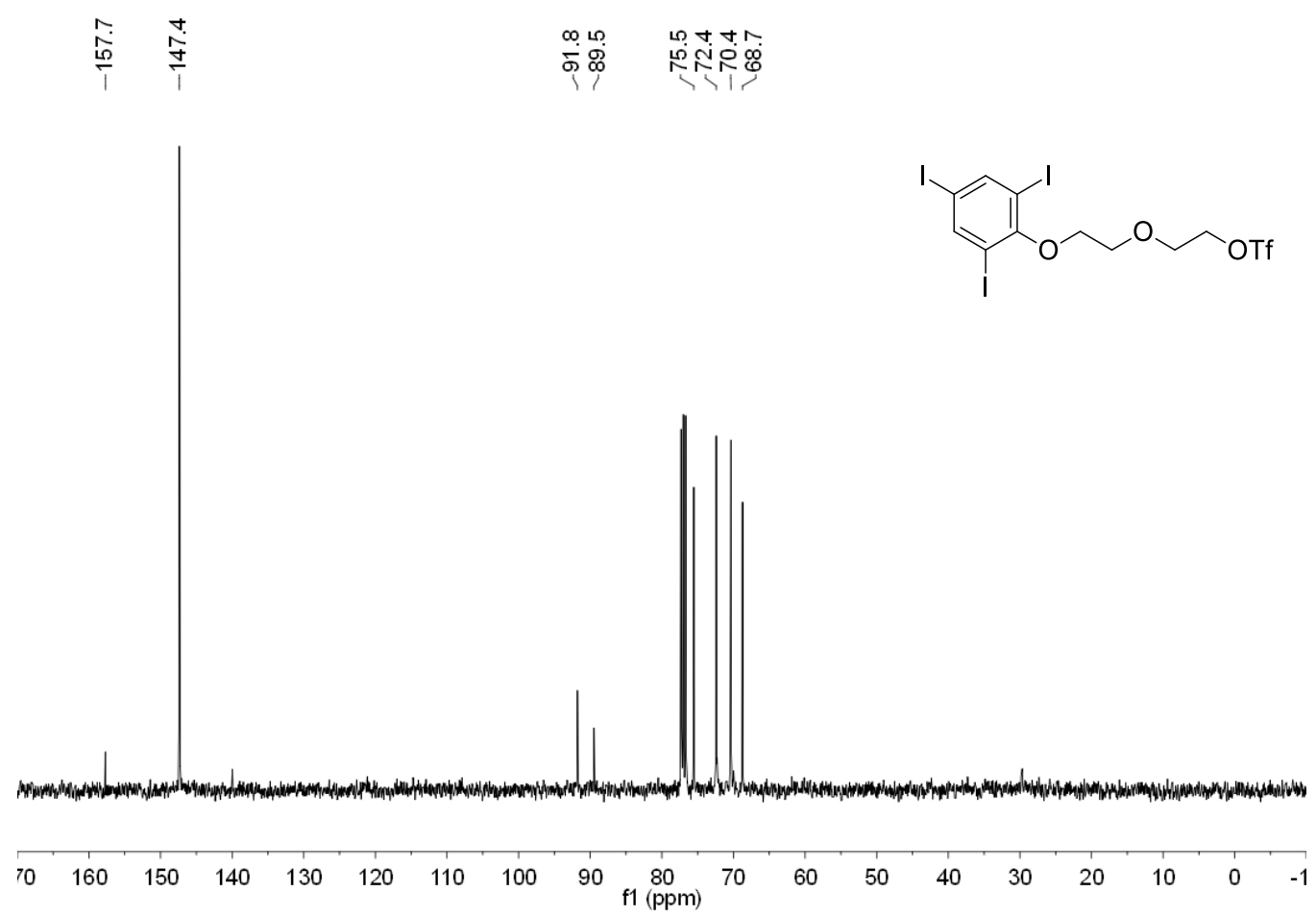

Spectrum 6. ${ }^{13} \mathrm{C}$ NMR (101 MHz, $\mathrm{CDCl}_{3}$ ) spectrum of 2-[2-(2,4,6-triiodophenoxy)ethoxy]ethyl trifluoromethanesulfonate. 


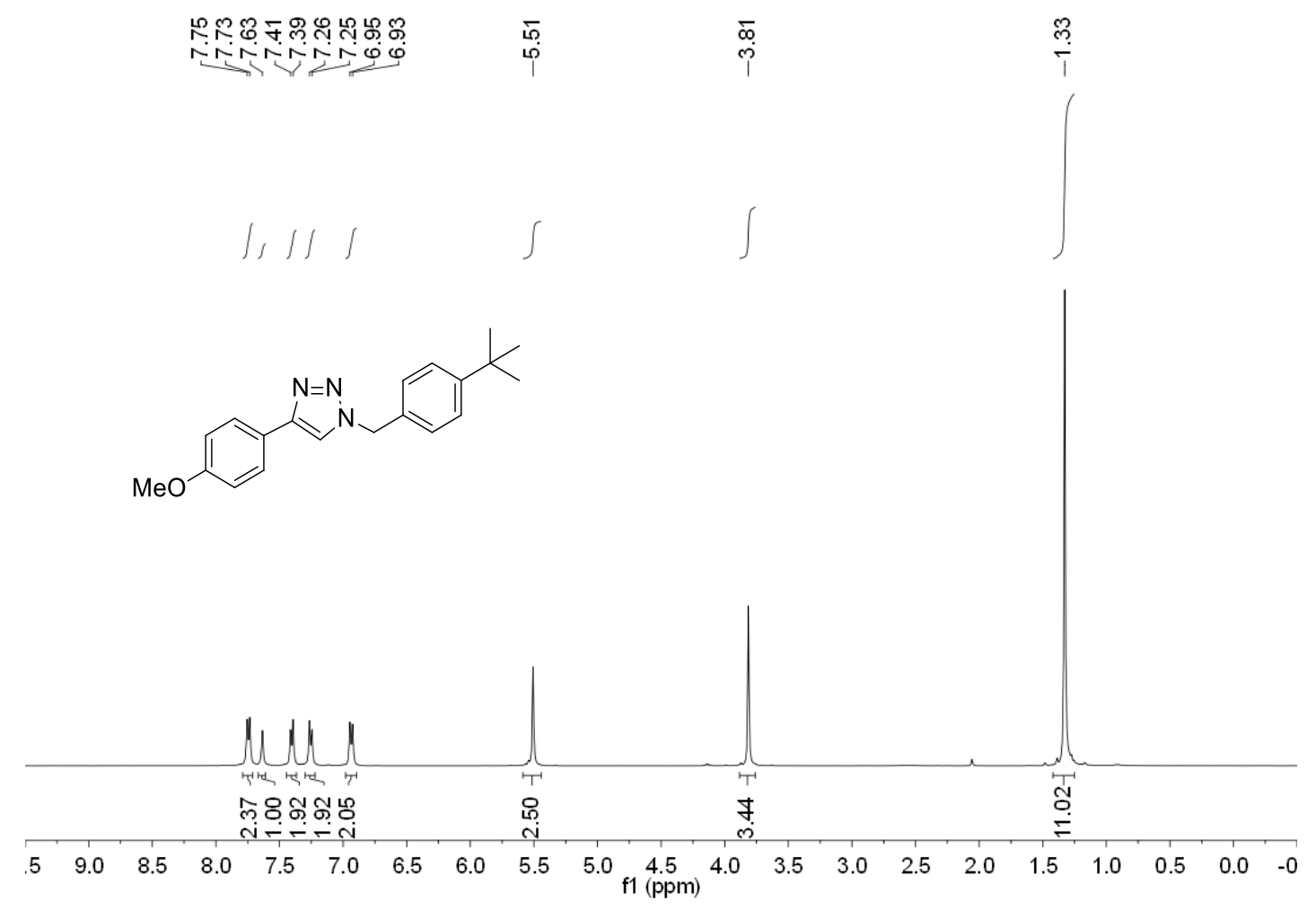

Spectrum 7. ${ }^{1} \mathrm{H}$ NMR (400 MHz, $\mathrm{CDCl}_{3}$ ) spectrum of compound $\mathbf{1 b}$.

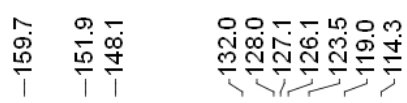

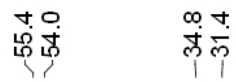
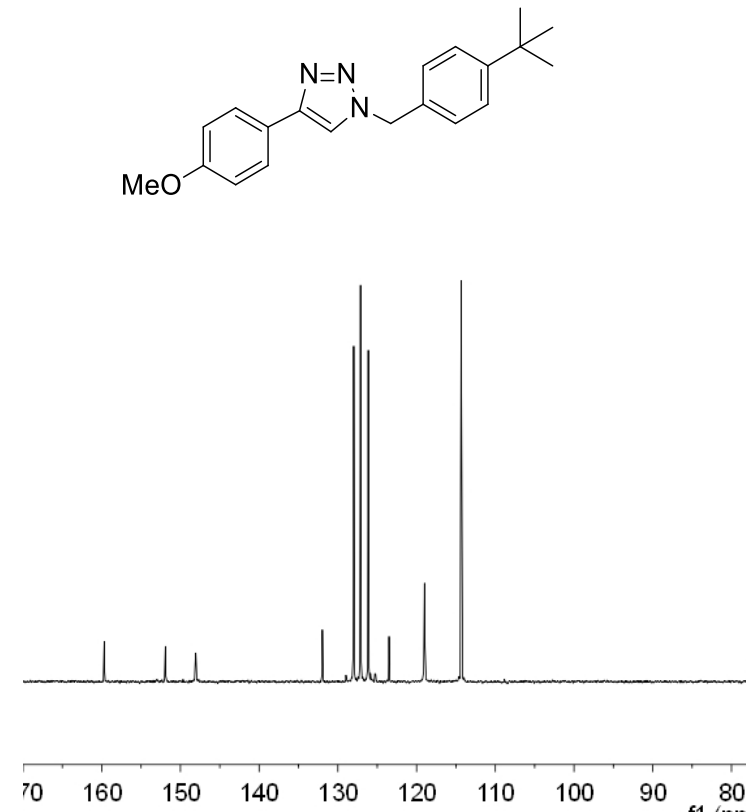

Spectrum 8. ${ }^{13} \mathrm{C}$ NMR (101 $\left.\mathrm{MHz}, \mathrm{CDCl}_{3}\right)$ spectrum of compound $\mathbf{1 b}$. 


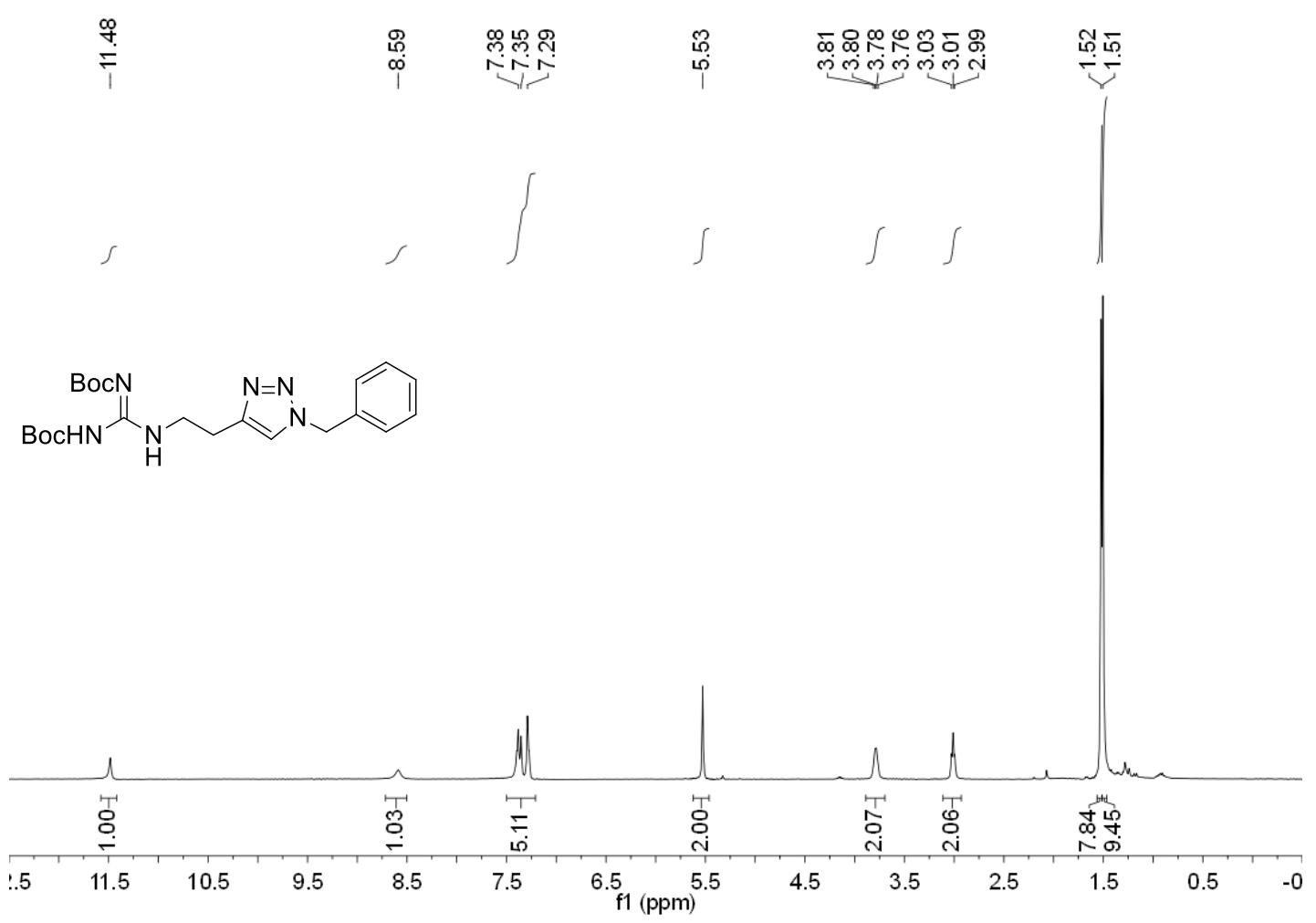

Spectrum 9. ${ }^{1} \mathrm{H}$ NMR (400 MHz, $\mathrm{CDCl}_{3}$ ) spectrum of compound $\mathbf{1 f}$.

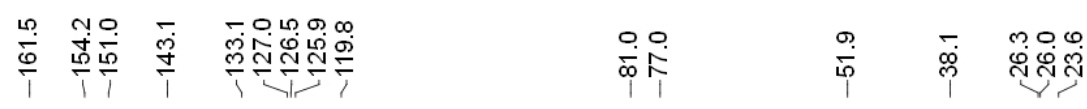<smiles>CC(C)(C)OC(=O)N=C(NCCc1cn(Cc2ccccc2)nn1)NC(=O)OC(C)(C)C</smiles>

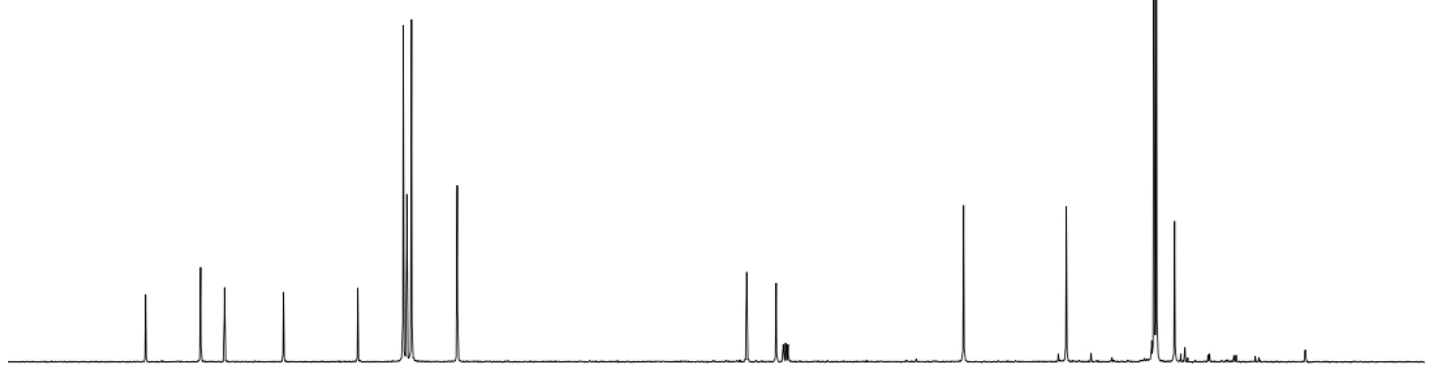

30

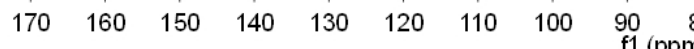

Spectrum 10. ${ }^{13} \mathrm{C}$ NMR $\left(101 \mathrm{MHz}, \mathrm{CDCl}_{3}\right)$ spectrum of compound $\mathbf{1 f}$. 


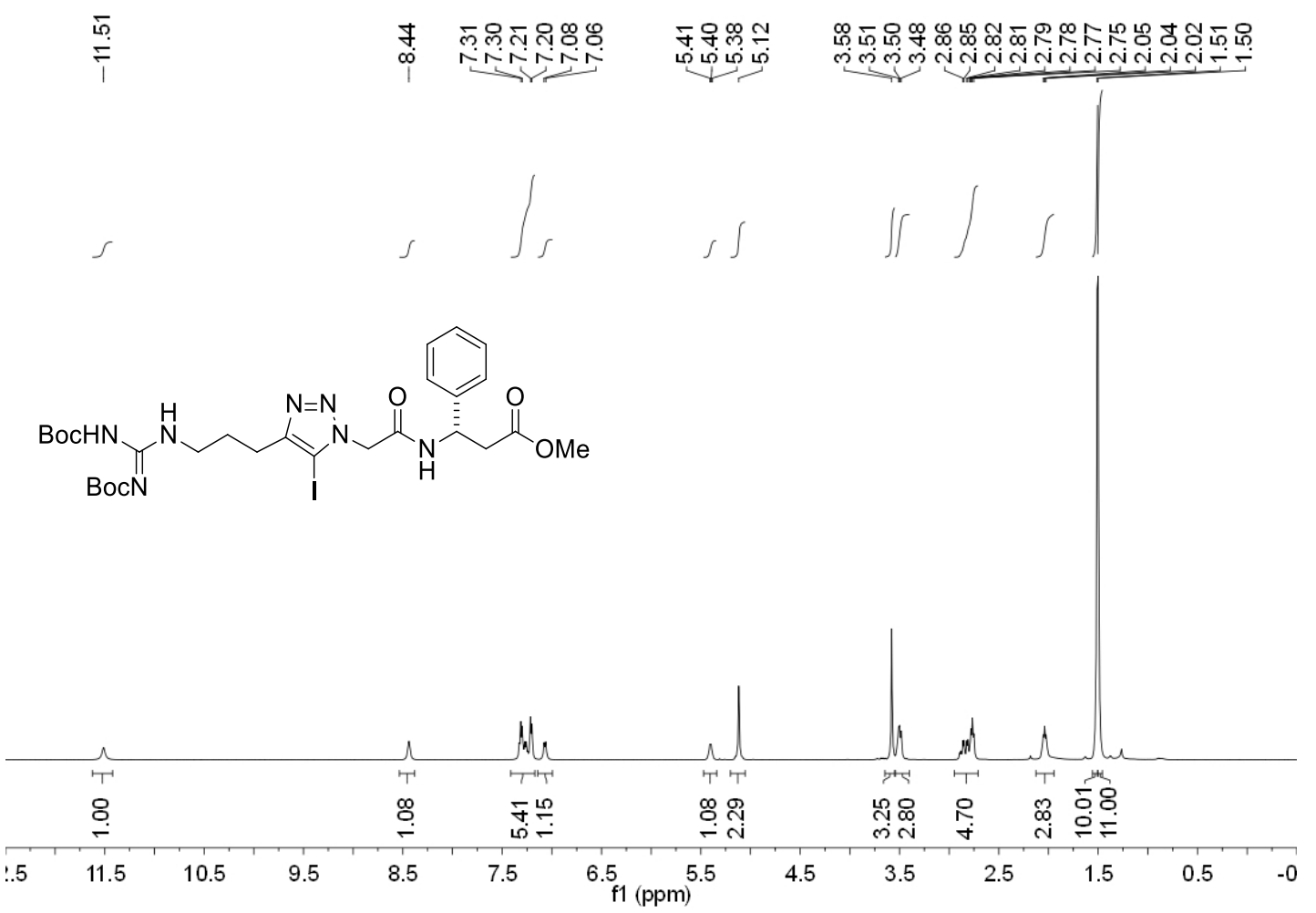

Spectrum 11. ${ }^{1} \mathrm{H}$ NMR $\left(500 \mathrm{MHz}, \mathrm{CDCl}_{3}\right)$ spectrum of compound $\mathbf{1 g}$.
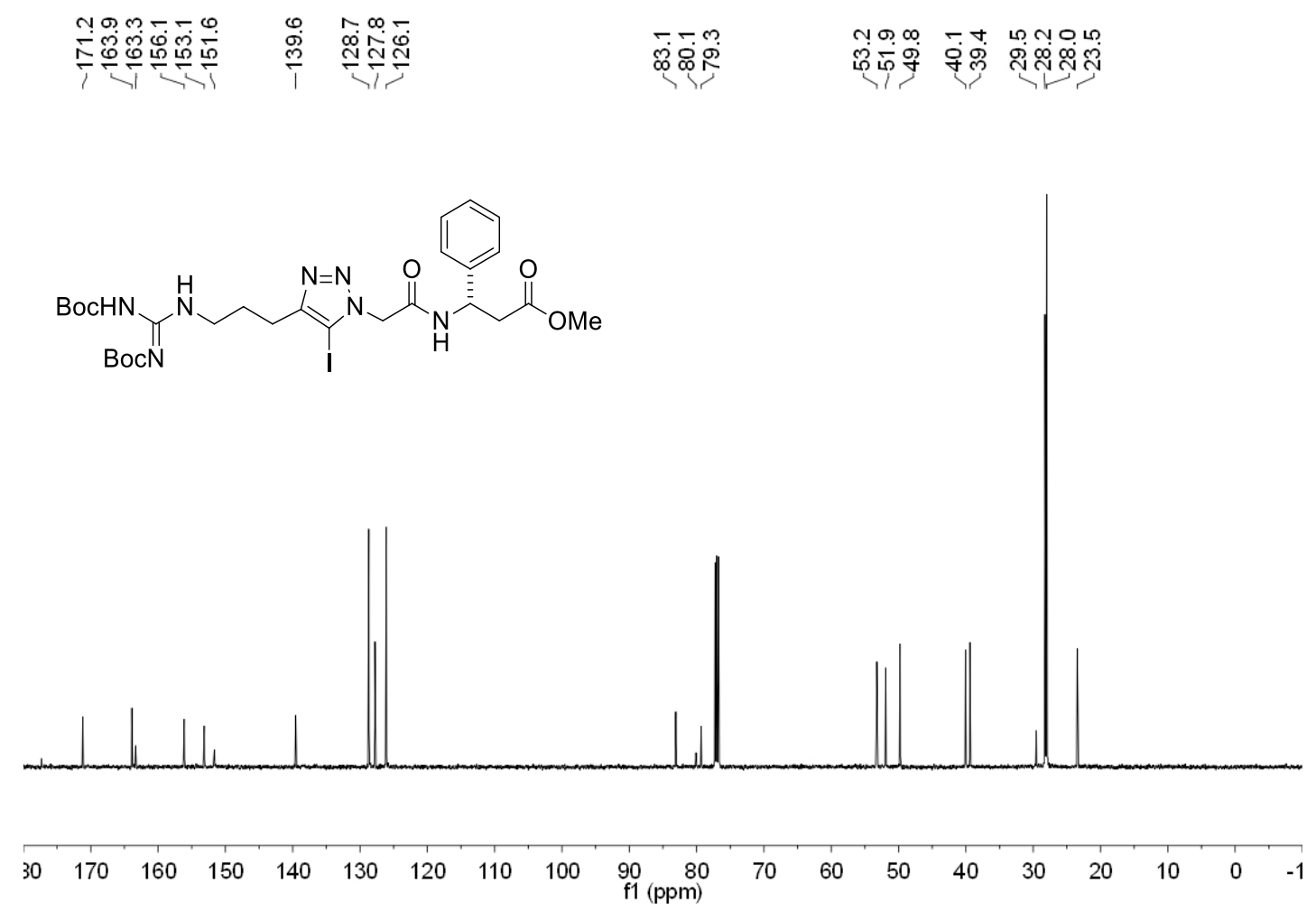

Spectrum 12. ${ }^{13} \mathrm{C}$ NMR $\left(126 \mathrm{MHz}, \mathrm{CDCl}_{3}\right)$ spectrum of compound $\mathbf{1 g}$. 


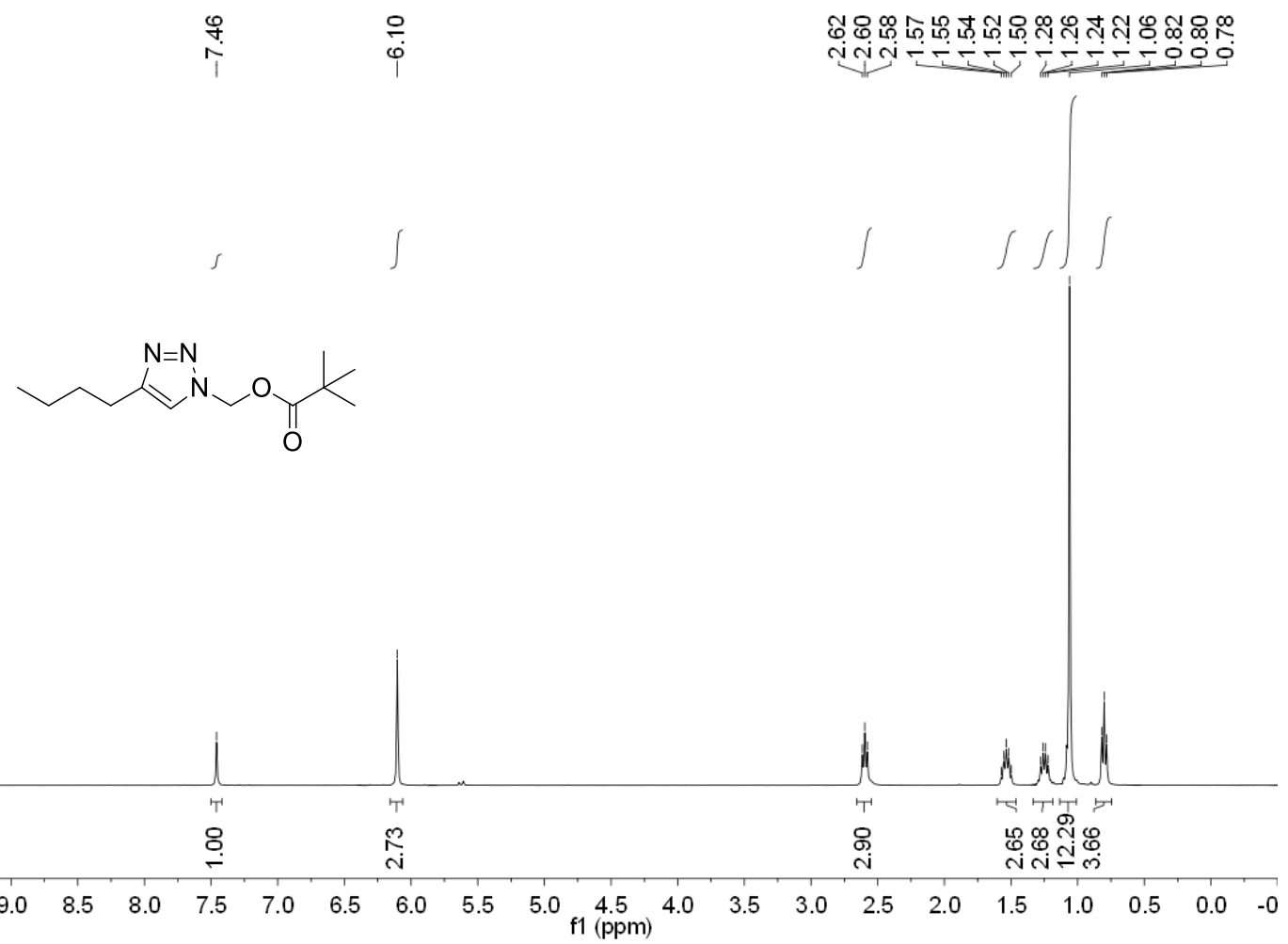

Spectrum 13. ${ }^{1} \mathrm{H}$ NMR (400 $\mathrm{MHz}, \mathrm{CDCl}_{3}$ ) spectrum of compound $\mathbf{1 j}$.

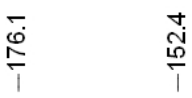

$\stackrel{\infty}{\infty} \stackrel{0}{\infty}$

:
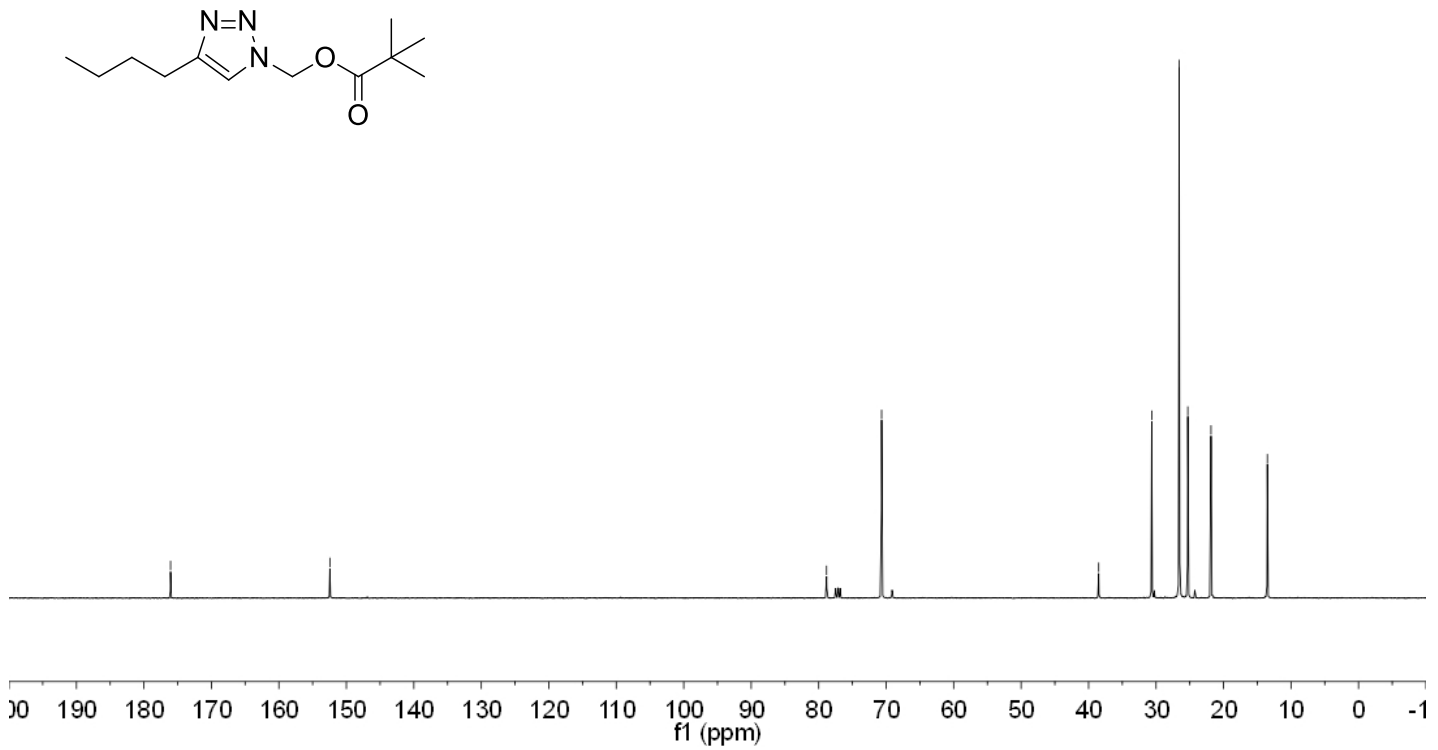

Spectrum 14. ${ }^{13} \mathrm{C}$ NMR $\left(126 \mathrm{MHz}, \mathrm{CDCl}_{3}\right)$ spectrum of compound $\mathbf{1 j}$. 


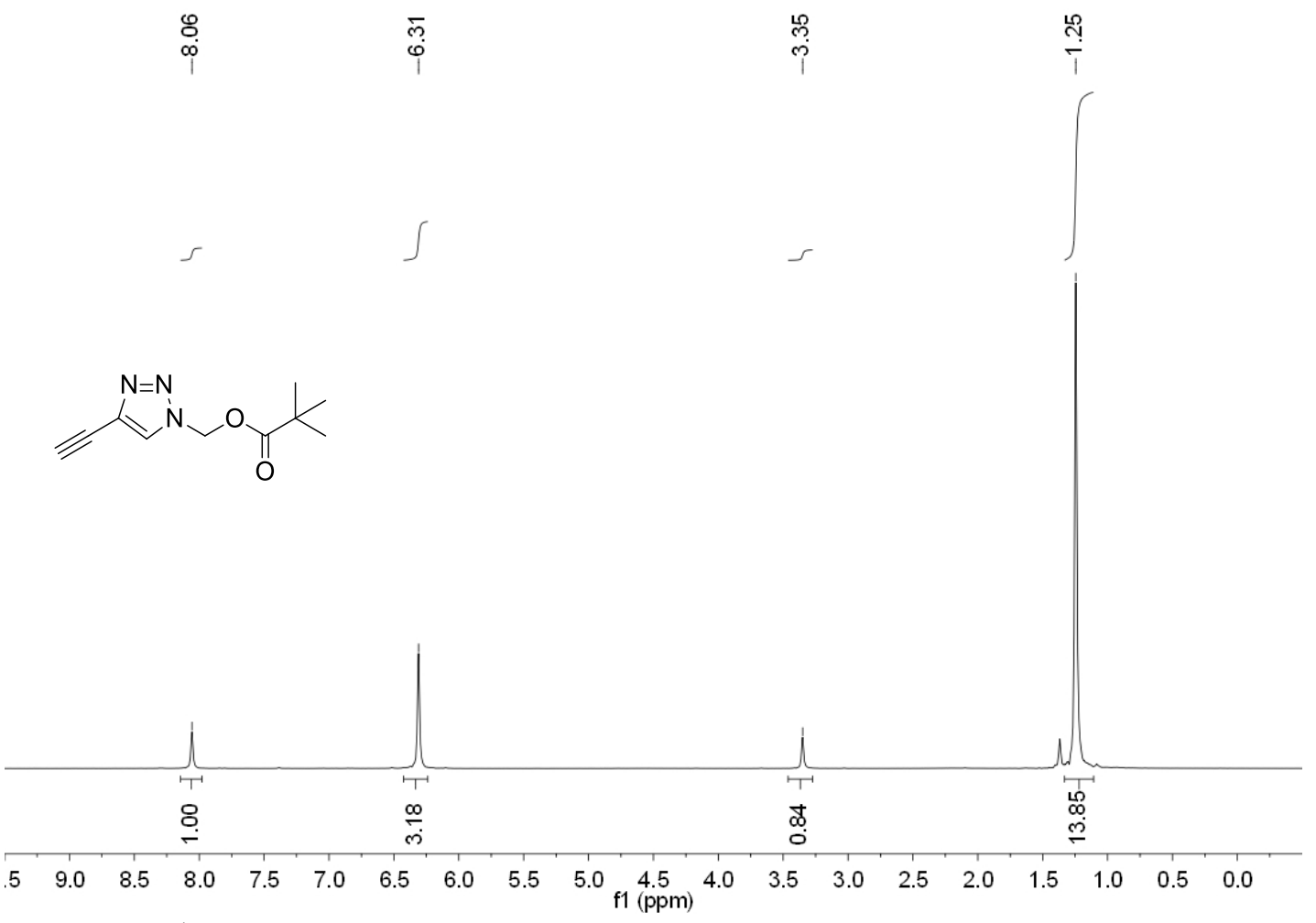

Spectrum 15. ${ }^{1} \mathrm{H}$ NMR (400 $\mathrm{MHz}, \mathrm{CDCl}_{3}$ ) spectrum of compound $\mathbf{1 k}$.

\begin{tabular}{|c|c|c|c|}
\hline$\stackrel{\stackrel{\hat{N}}{\mathrm{~N}}}{=}$ & $\frac{\stackrel{3}{+}}{\dot{q}}$ & $\frac{\text { Ñ}}{\stackrel{\text { }}{T}}$ & 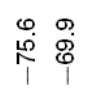 \\
\hline
\end{tabular}<smiles>C#Cc1cn(COC(=O)C(C)(C)C)nn1</smiles>
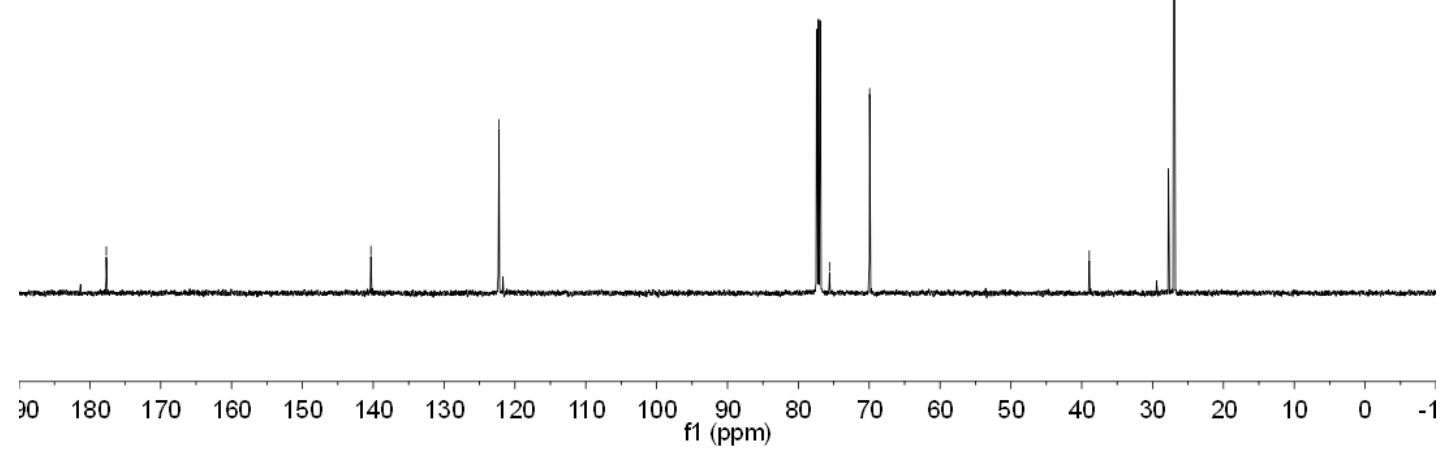

Spectrum 16. ${ }^{13} \mathrm{C}$ NMR $\left(126 \mathrm{MHz}, \mathrm{CDCl}_{3}\right)$ spectrum of compound $\mathbf{1 k}$. 


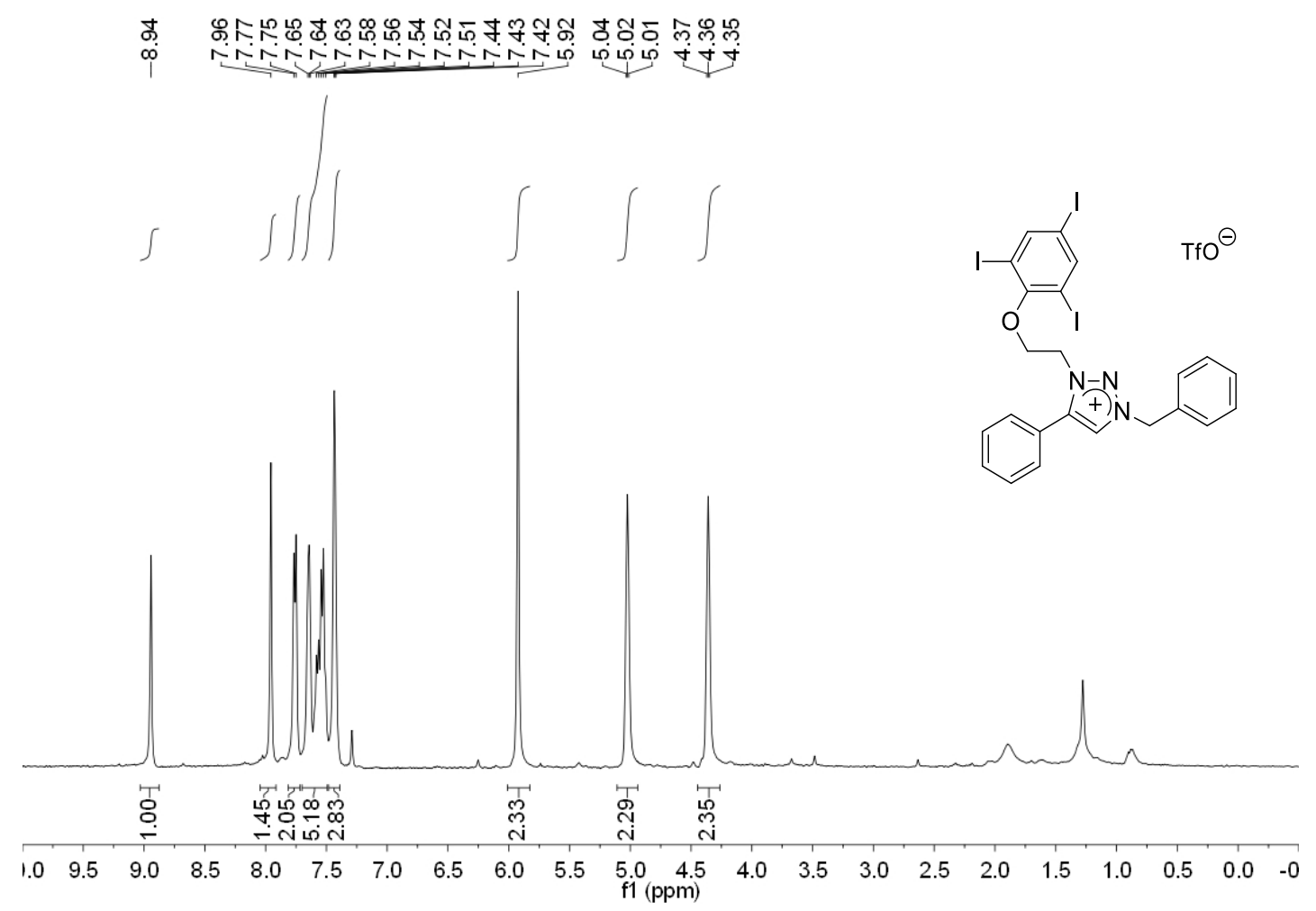

Spectrum 17. ${ }^{1} \mathrm{H}$ NMR $\left(400 \mathrm{MHz}, \mathrm{CDCl}_{3}\right)$ spectrum of compound 2a.

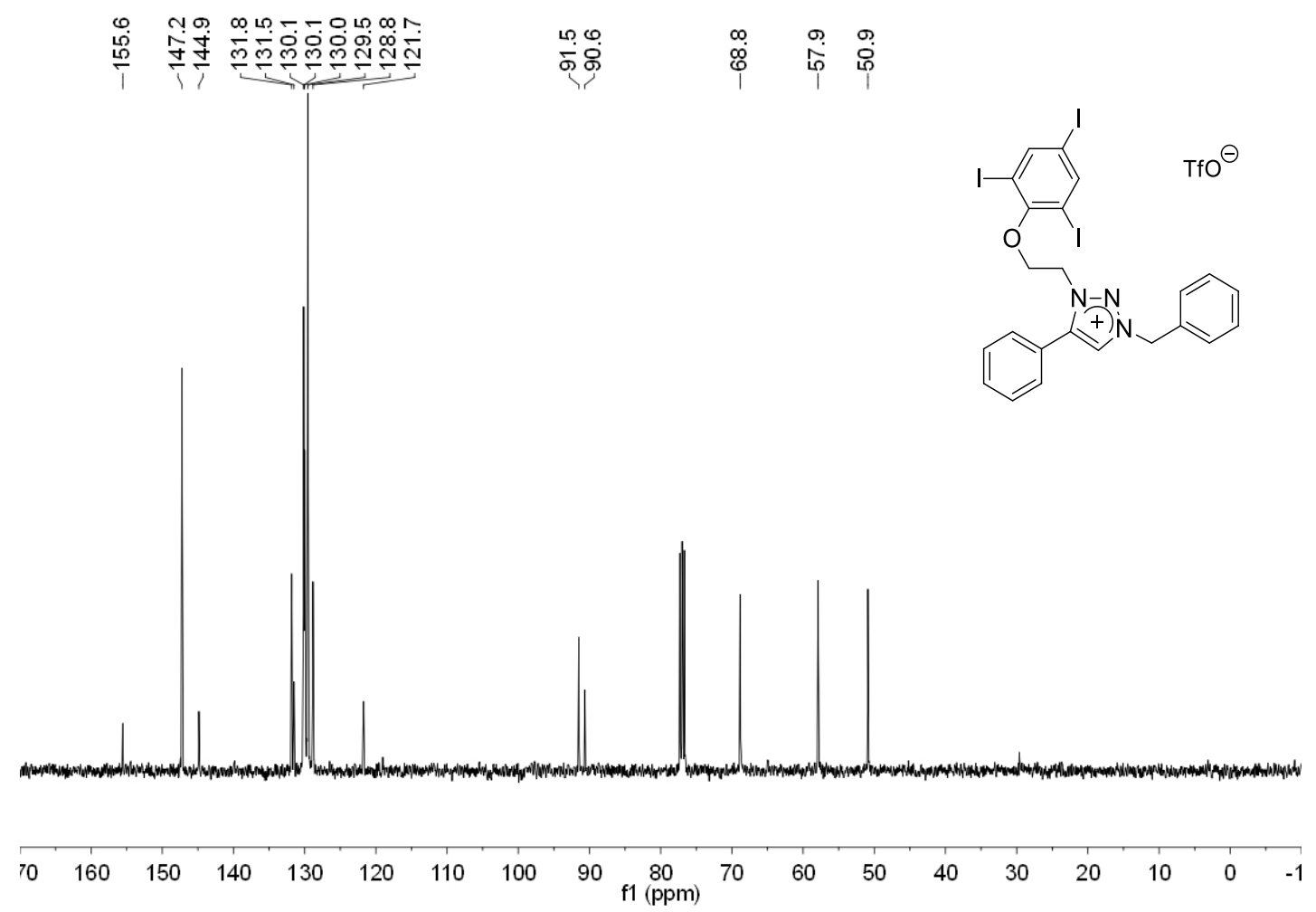

Spectrum 18. ${ }^{13} \mathrm{C}$ NMR $\left(101 \mathrm{MHz}, \mathrm{CDCl}_{3}\right)$ spectrum of compound $2 \mathbf{a}$. 


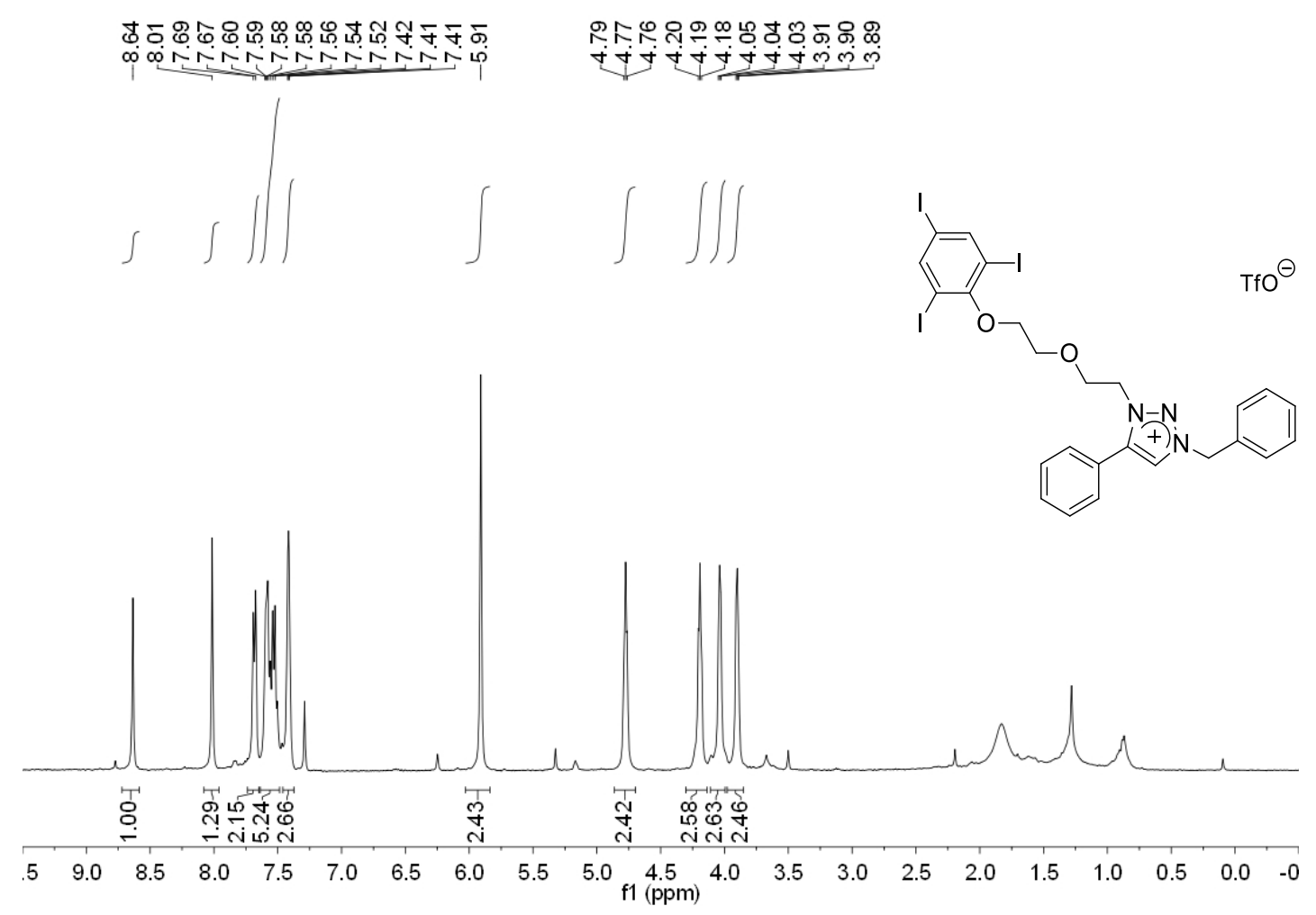

Spectrum 19. ${ }^{1} \mathrm{H}$ NMR $\left(400 \mathrm{MHz}, \mathrm{CDCl}_{3}\right)$ spectrum of compound $\mathbf{2 b}$.
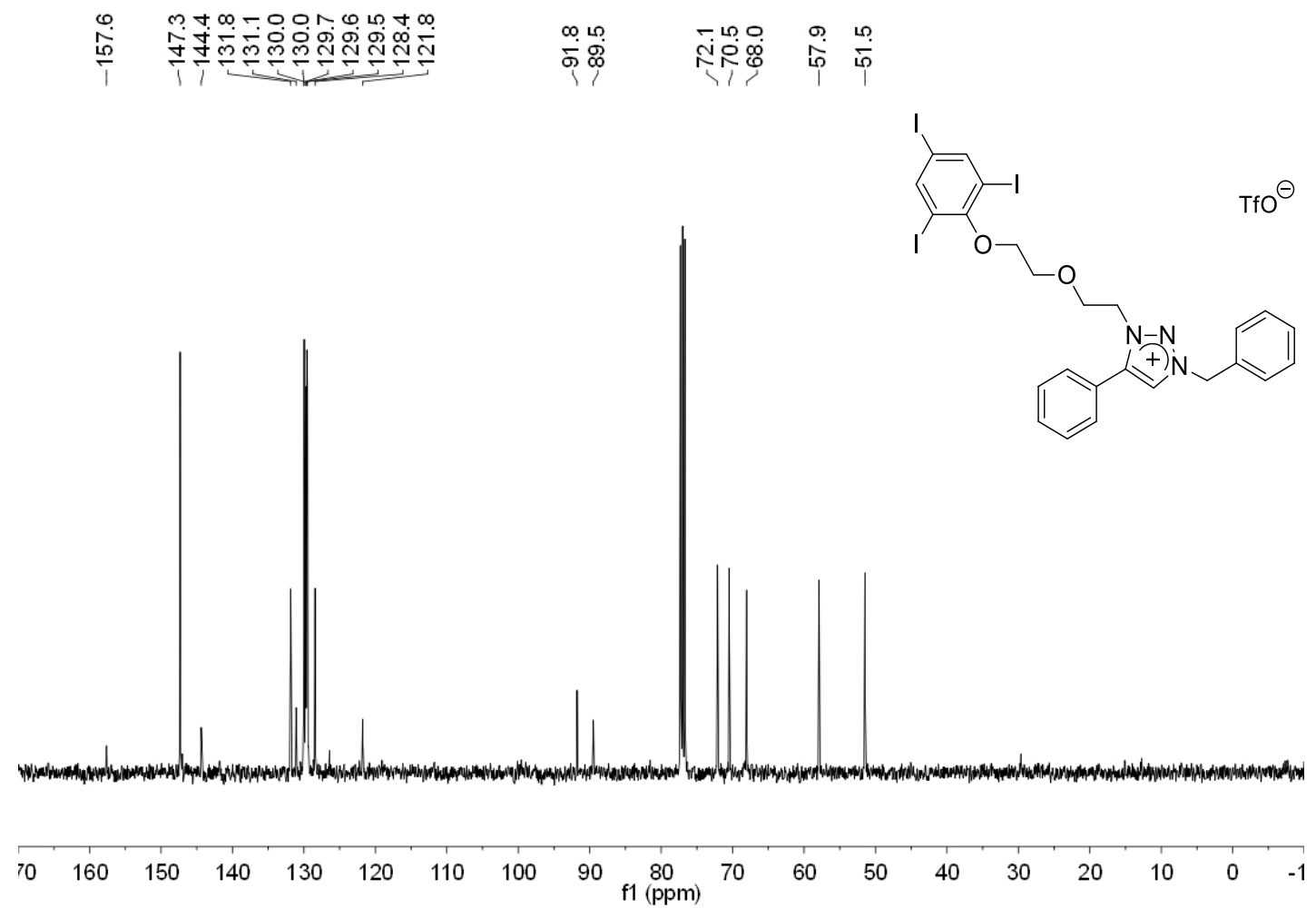

Spectrum 20. ${ }^{13} \mathrm{C}$ NMR $\left(101 \mathrm{MHz}, \mathrm{CDCl}_{3}\right)$ spectrum of compound $\mathbf{2 b}$. 


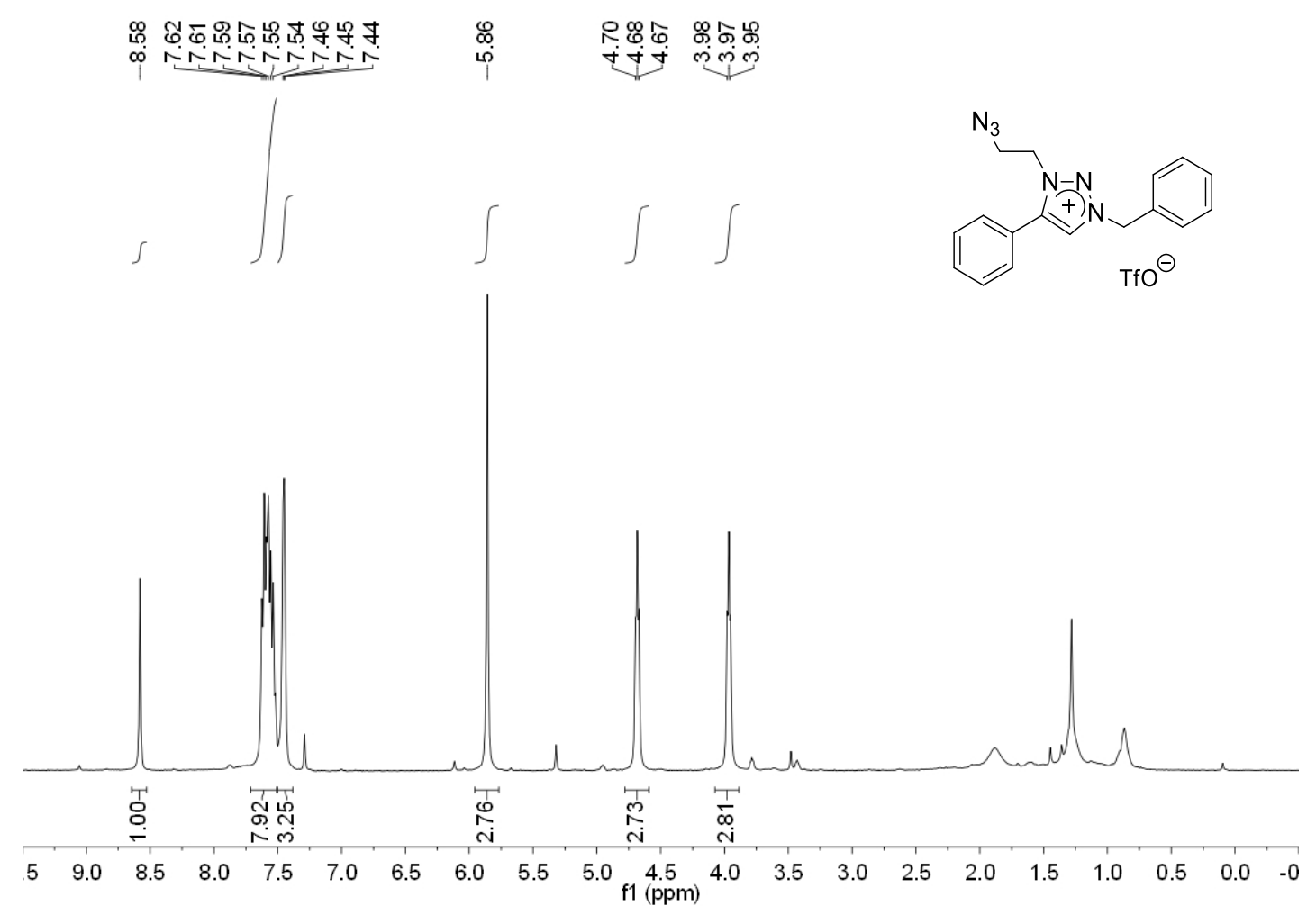

Spectrum 21. ${ }^{1} \mathrm{H}$ NMR ( $400 \mathrm{MHz}, \mathrm{CDCl}_{3}$ ) spectrum of compound $\mathbf{2 c}$.

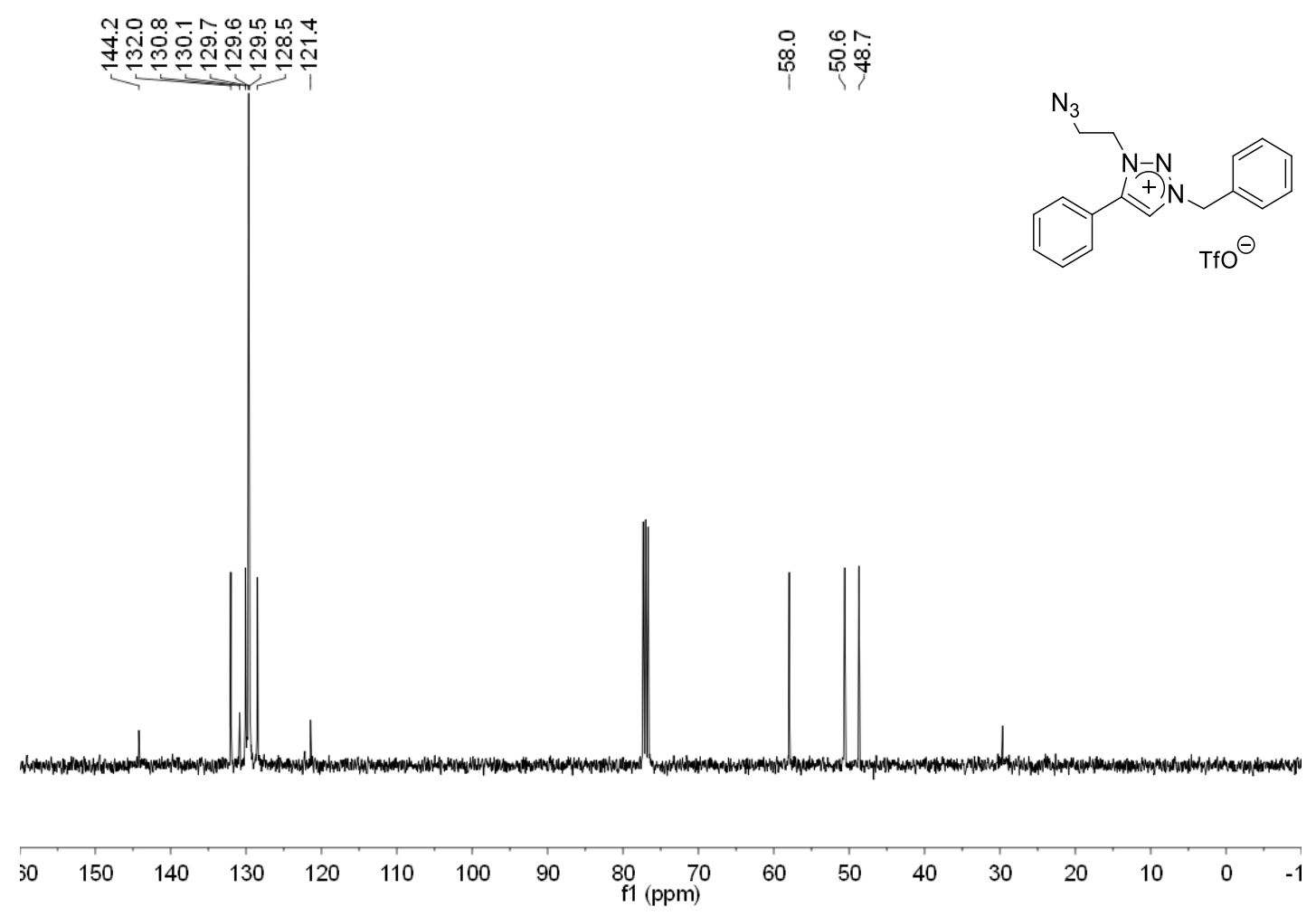

Spectrum 22. ${ }^{13} \mathrm{C}$ NMR (101 MHz, $\mathrm{CDCl}_{3}$ ) spectrum of compound 2c. 


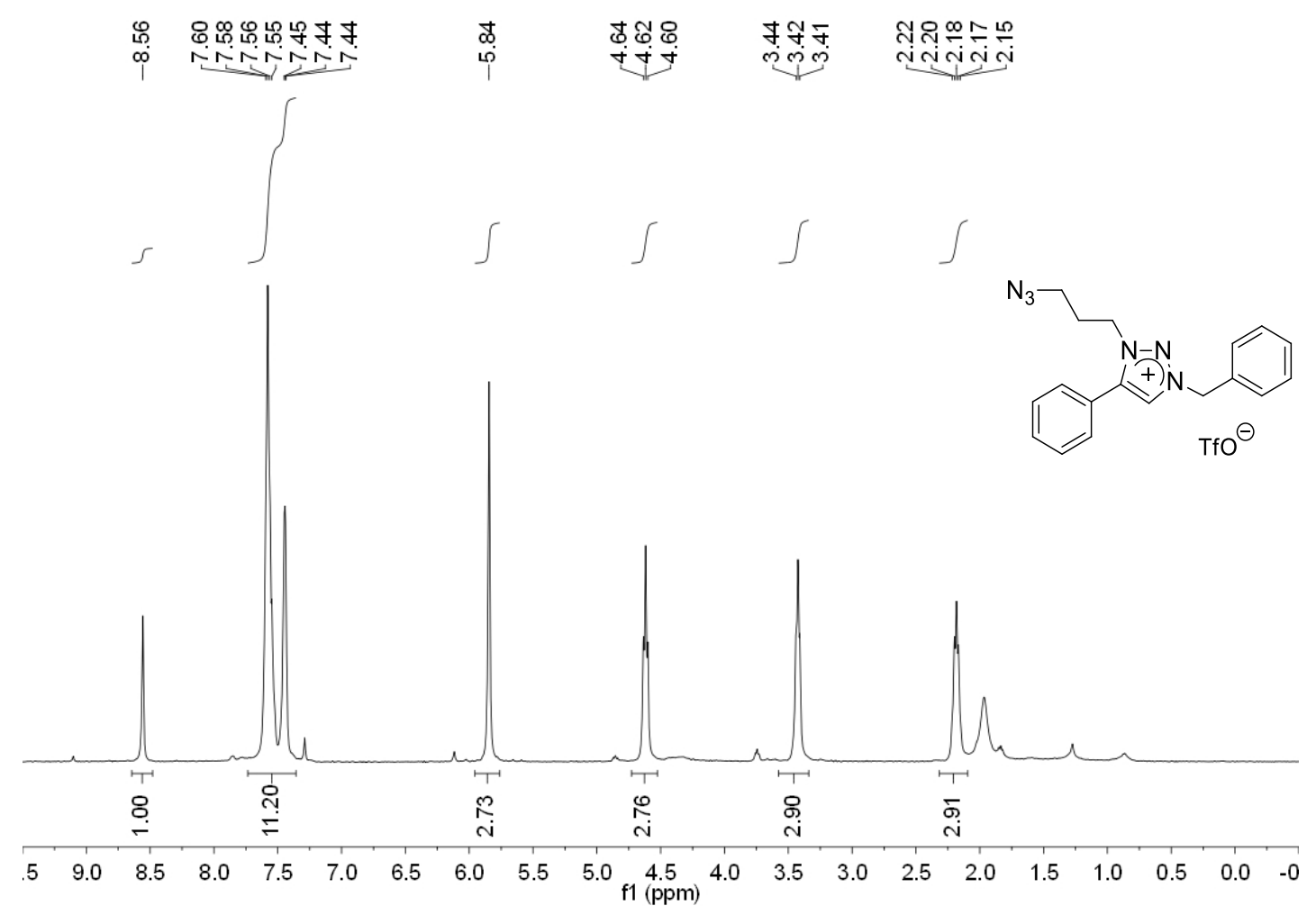

Spectrum 23. ${ }^{1} \mathrm{H}$ NMR $\left(400 \mathrm{MHz}, \mathrm{CDCl}_{3}\right)$ spectrum of compound $\mathbf{2 d}$.
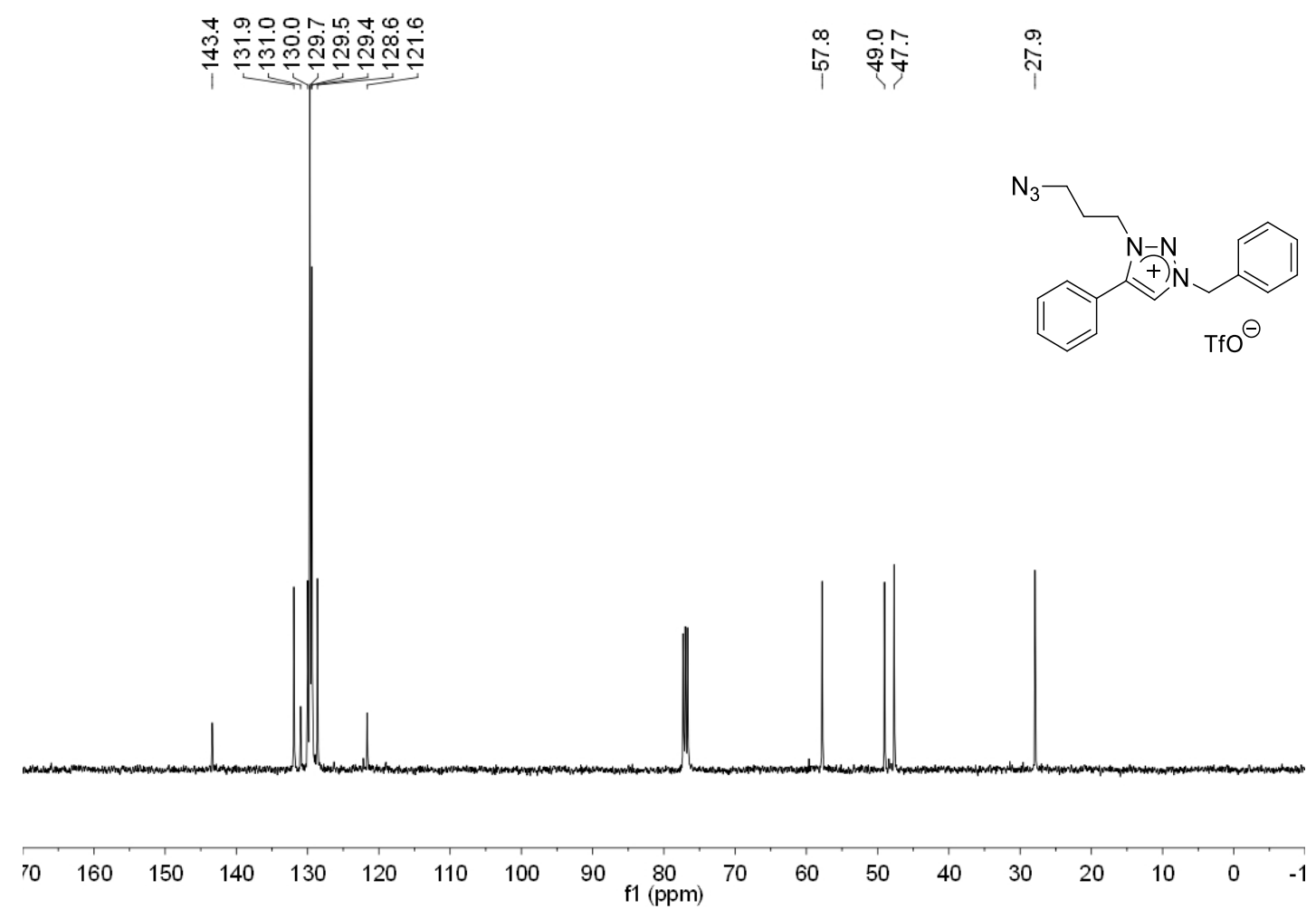

Spectrum 24. ${ }^{13} \mathrm{C}$ NMR $\left(101 \mathrm{MHz}, \mathrm{CDCl}_{3}\right)$ spectrum of compound $2 \mathbf{d}$. 


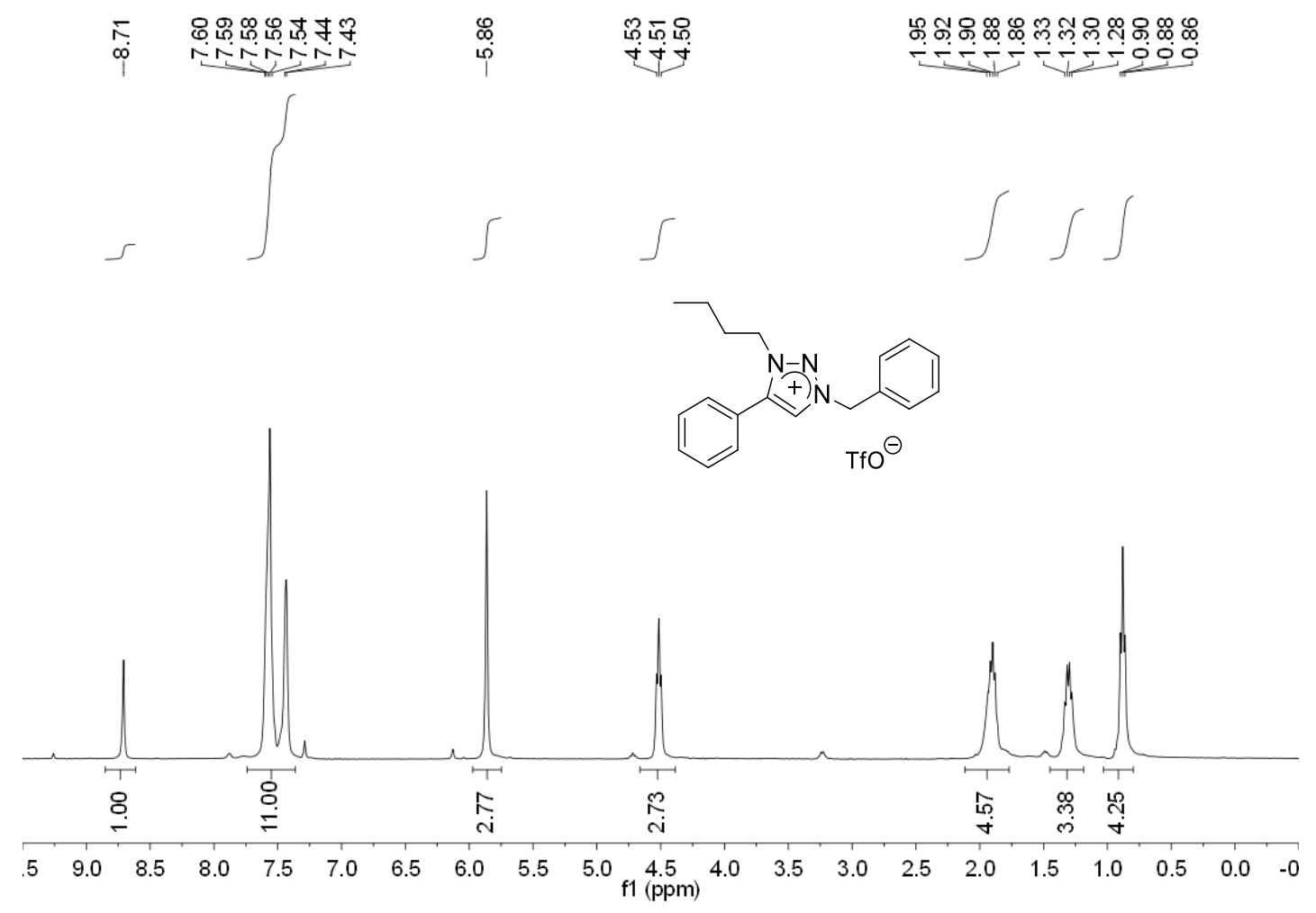

Spectrum 25. ${ }^{1} \mathrm{H}$ NMR (400 MHz, $\mathrm{CDCl}_{3}$ ) spectrum of compound $2 \mathbf{e}$.

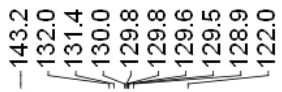

䒢

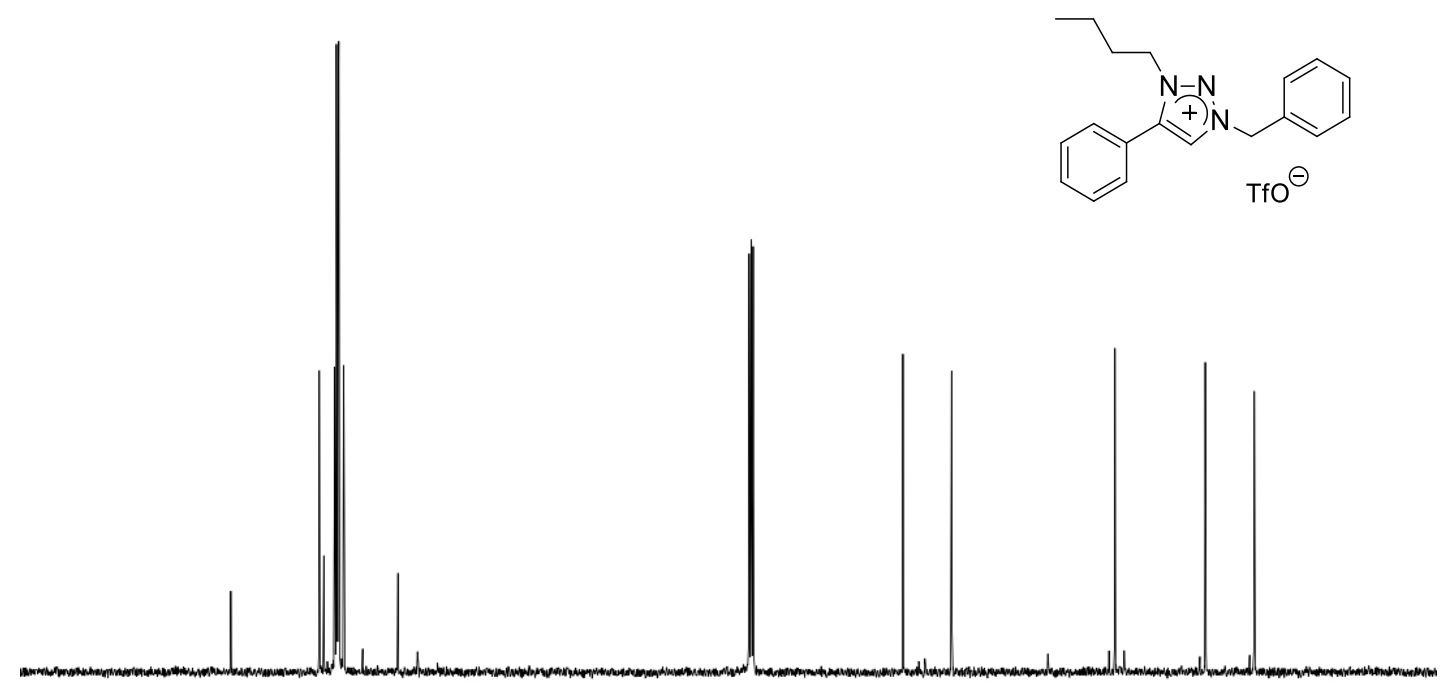

\begin{tabular}{lllllllllllllllllll}
\hline 0 & 160 & 150 & 140 & 130 & 120 & 110 & 100 & 90 & $\begin{array}{c}80 \\
\mathrm{f} 1(\mathrm{ppm})\end{array}$ & 70 & 60 & 50 & 40 & 30 & 20 & 10 & 0 & -1
\end{tabular}

Spectrum 26. ${ }^{13} \mathrm{C}$ NMR $\left(126 \mathrm{MHz}, \mathrm{CDCl}_{3}\right)$ spectrum of compound $2 \mathrm{e}$. 


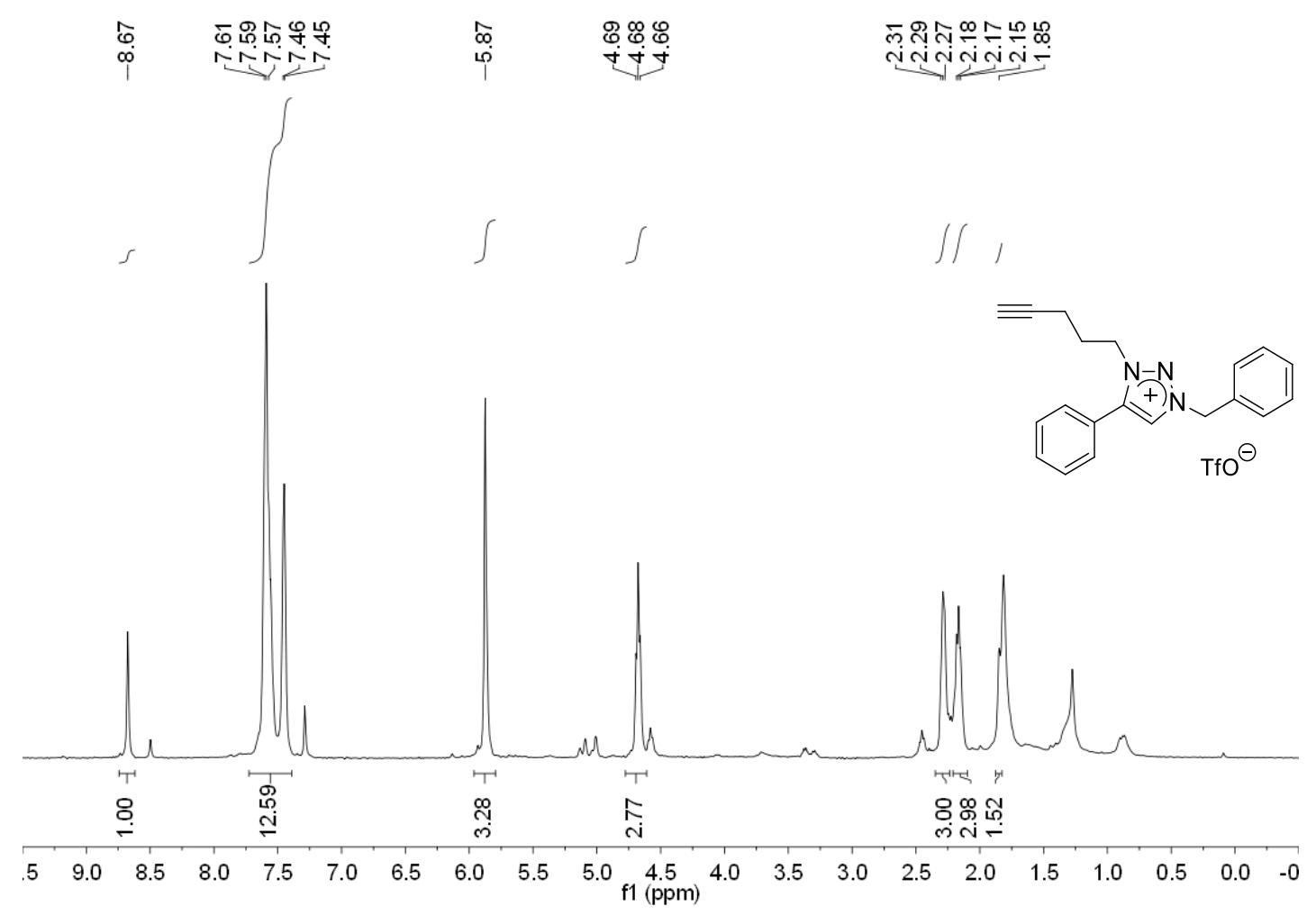

Spectrum 27. ${ }^{1} \mathrm{H}$ NMR $\left(400 \mathrm{MHz}, \mathrm{CDCl}_{3}\right)$ spectrum of compound $\mathbf{2 f}$.
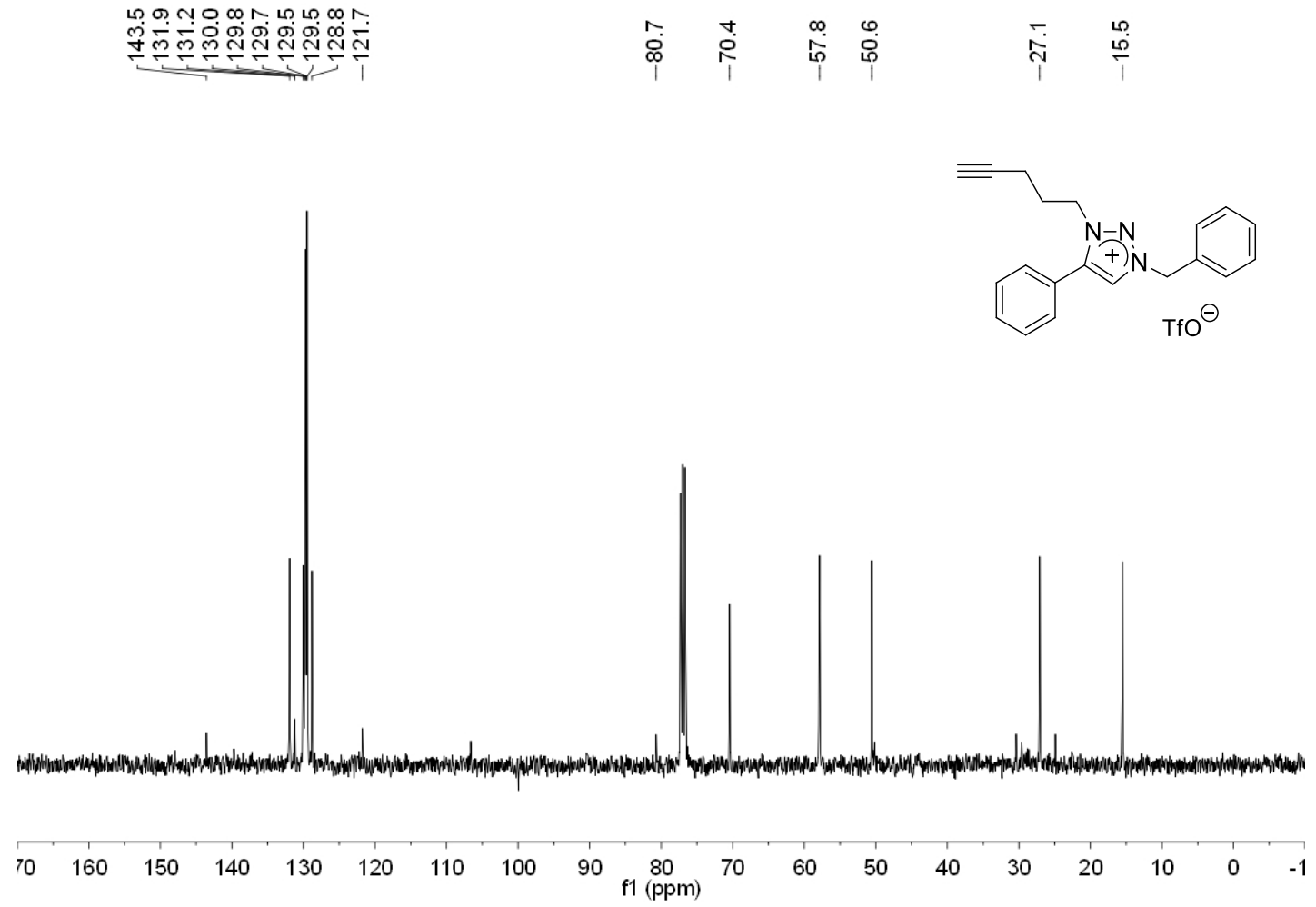

Spectrum 28. ${ }^{13} \mathrm{C}$ NMR $\left(101 \mathrm{MHz}, \mathrm{CDCl}_{3}\right)$ spectrum of compound $2 \mathbf{f}$. 


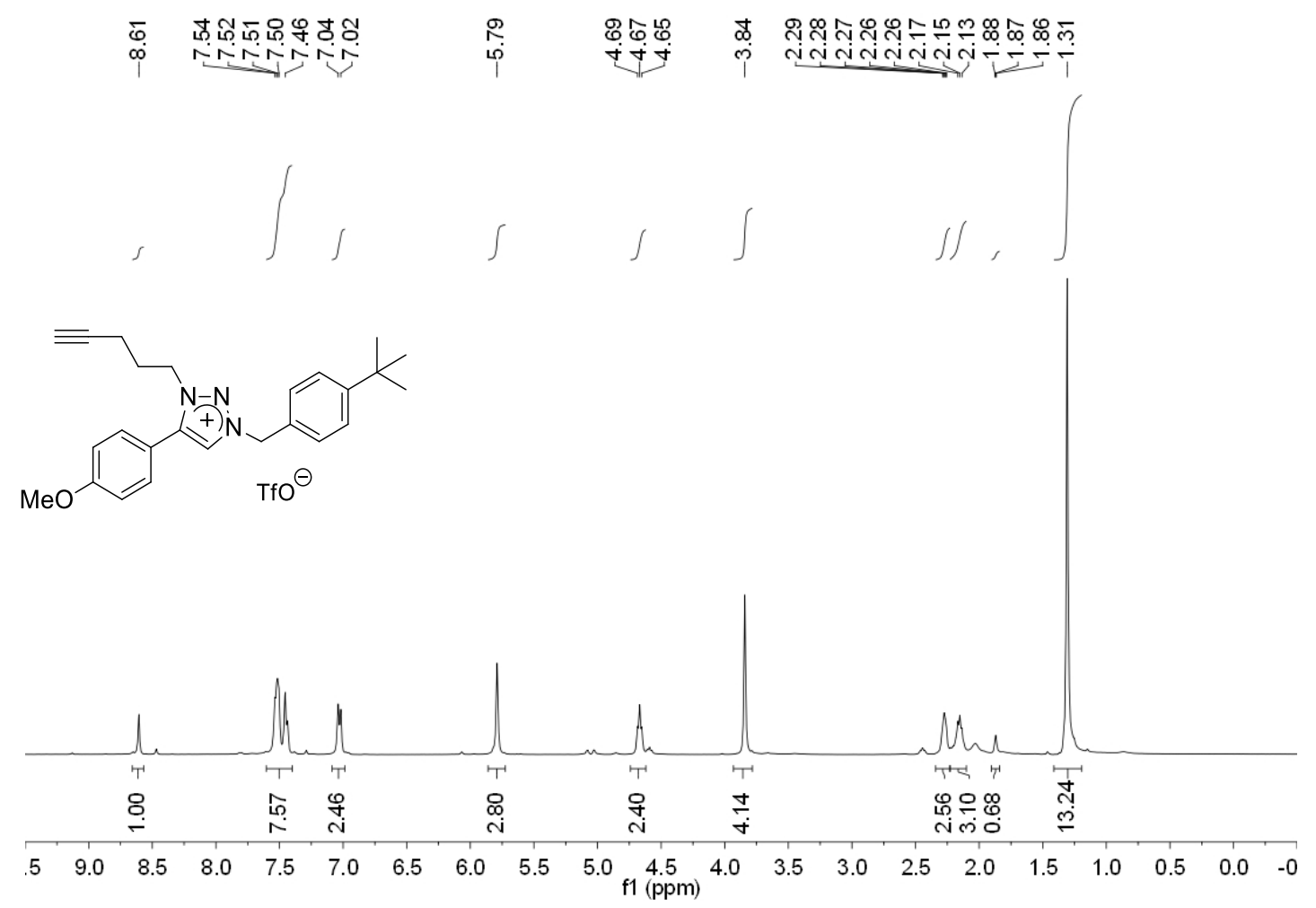

Spectrum 29. ${ }^{1} \mathrm{H}$ NMR (400 MHz, $\left.\mathrm{CDCl}_{3}\right)$ spectrum of compound $\mathbf{2 g}$.

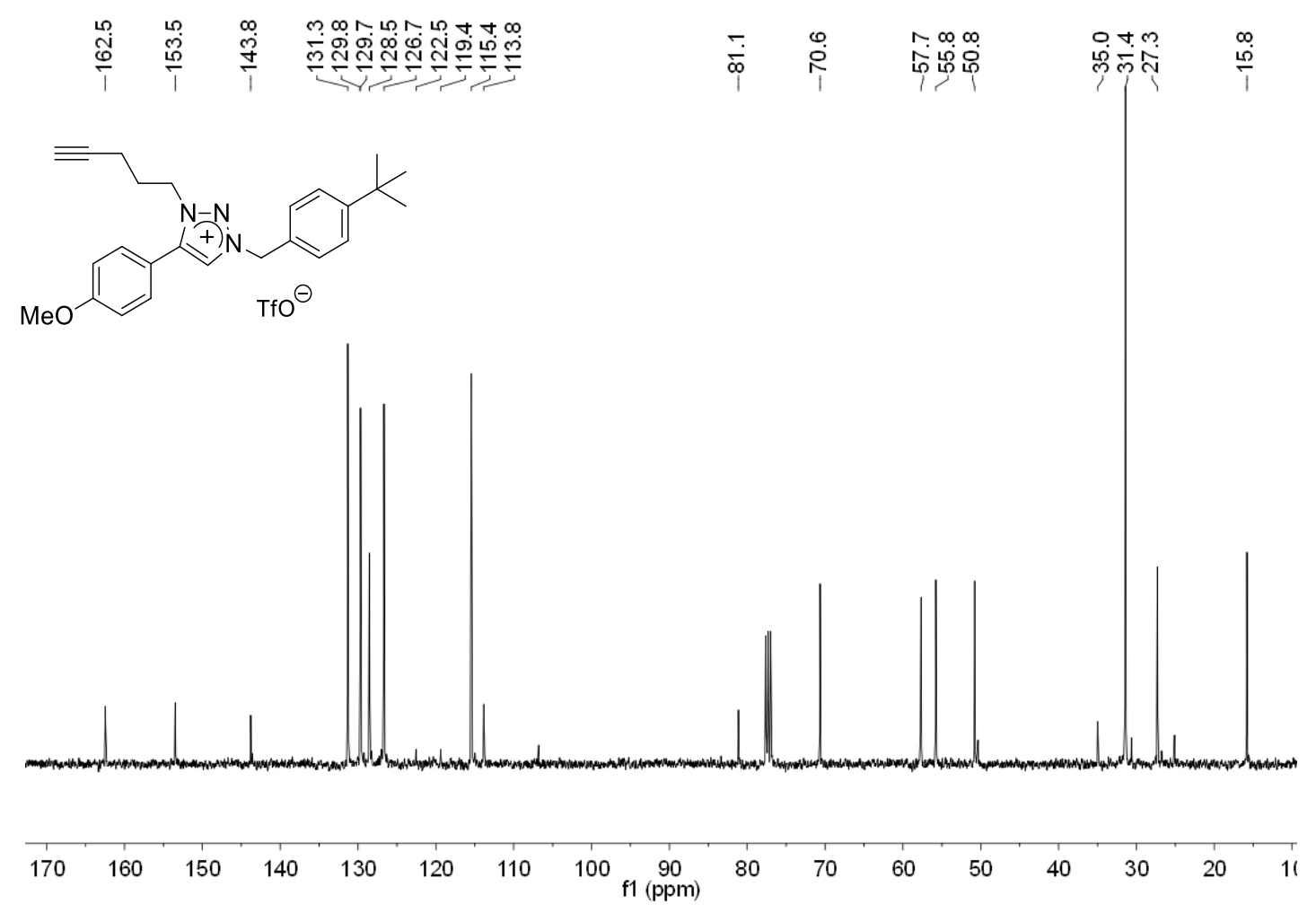

Spectrum 30. ${ }^{13} \mathrm{C}$ NMR (101 MHz, $\left.\mathrm{CDCl}_{3}\right)$ spectrum of compound $\mathbf{2 g}$. 


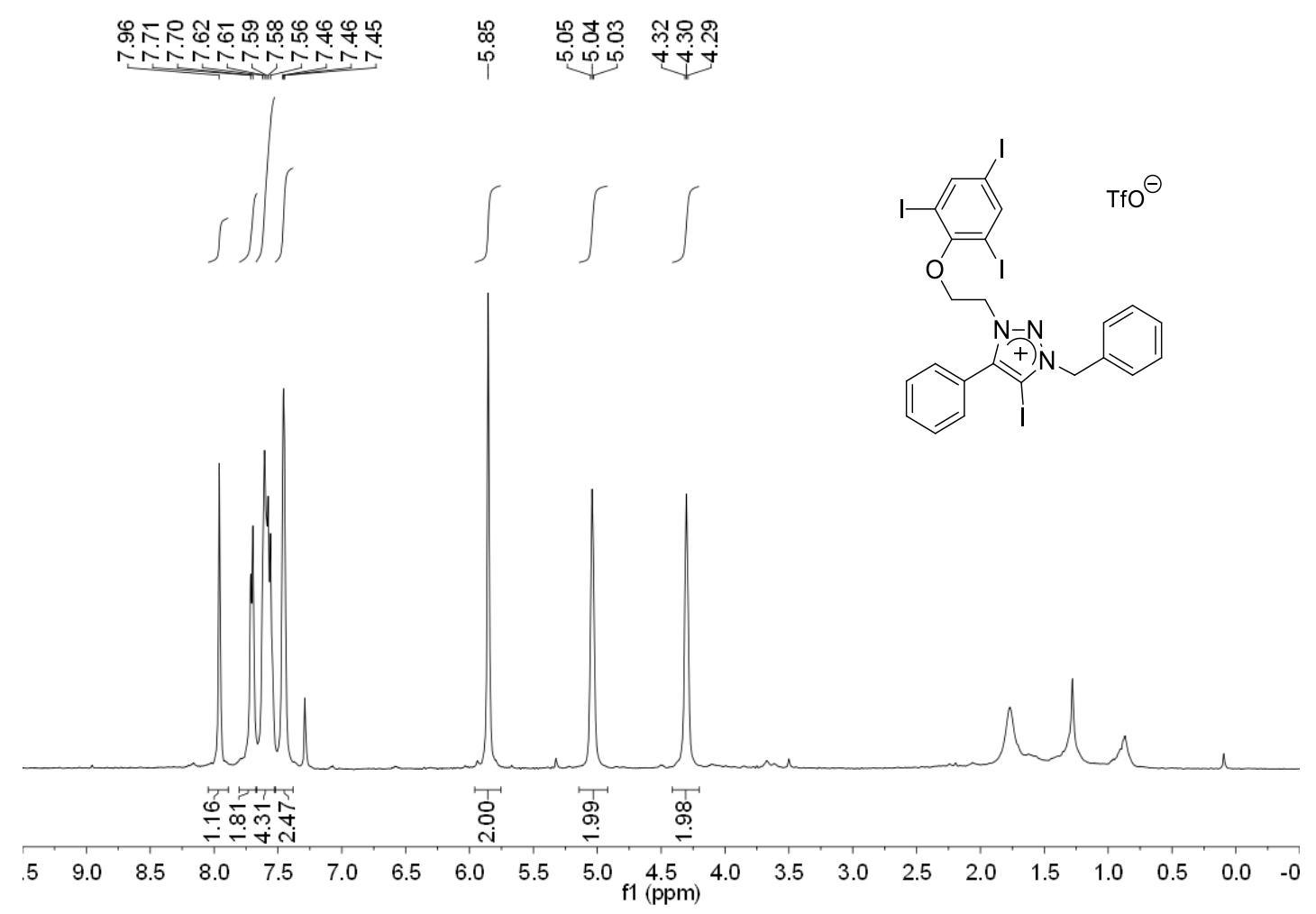

Spectrum 31. ${ }^{1} \mathrm{H}$ NMR $\left(400 \mathrm{MHz}, \mathrm{CDCl}_{3}\right)$ spectrum of compound $\mathbf{2 h}$.

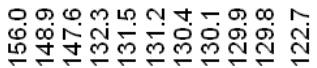

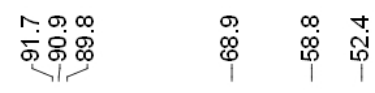

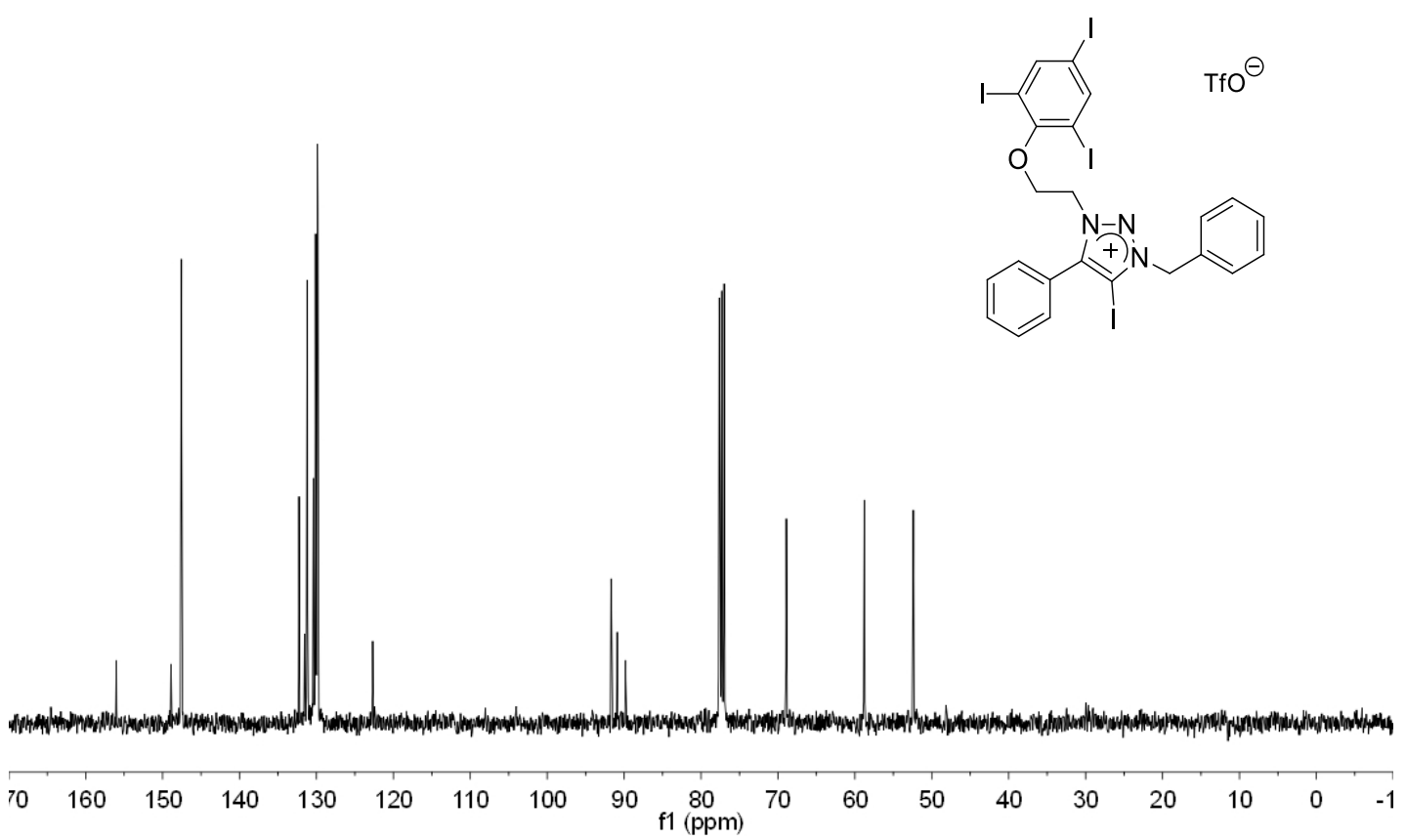

Spectrum 32. ${ }^{13} \mathrm{C}$ NMR $\left(101 \mathrm{MHz}, \mathrm{CDCl}_{3}\right.$ ) spectrum of compound $\mathbf{2 h}$. 


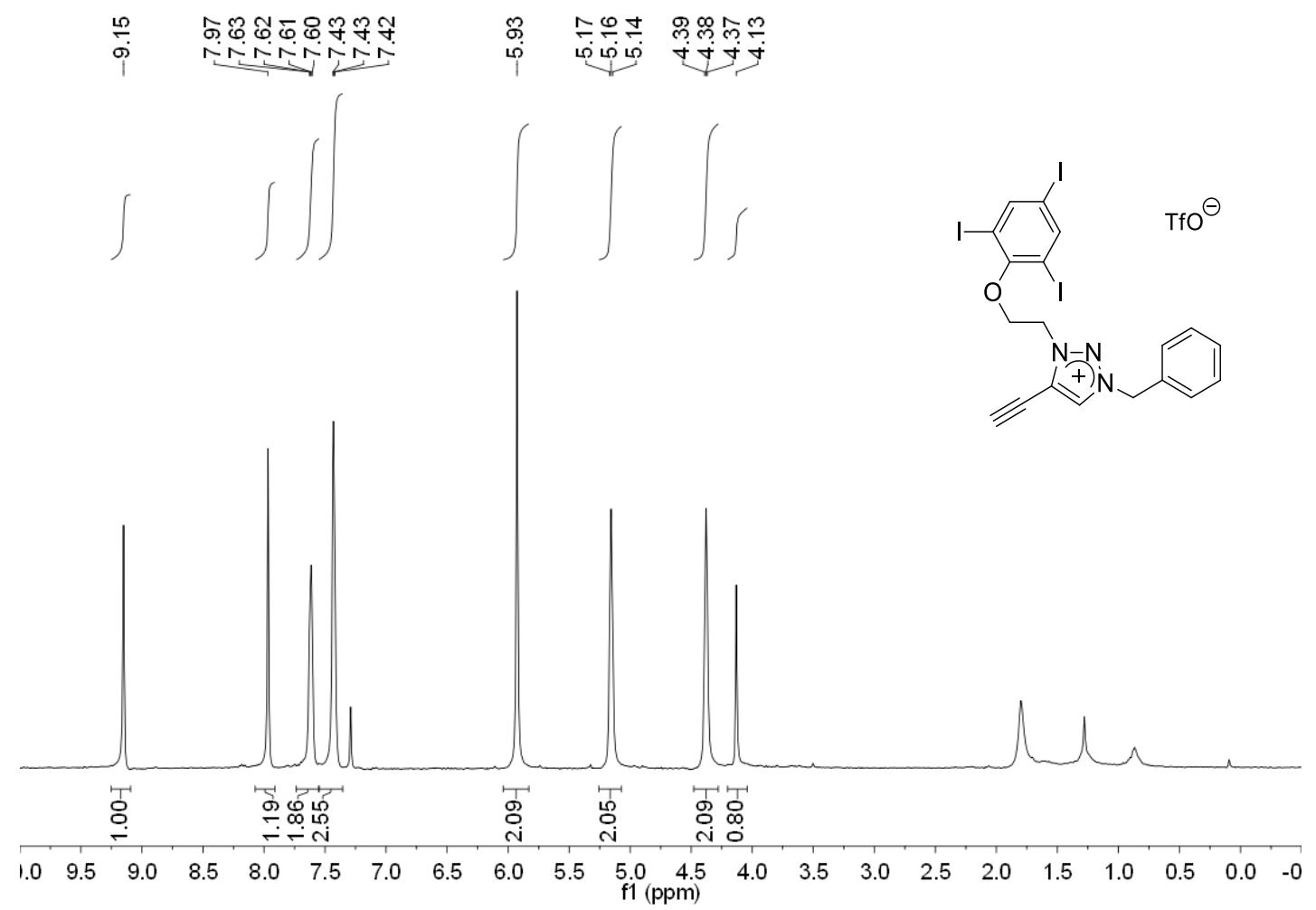

Spectrum 33. ${ }^{1} \mathrm{H}$ NMR ( $\left.400 \mathrm{MHz}, \mathrm{CDCl}_{3}\right)$ spectrum of compound $\mathbf{2 i}$.
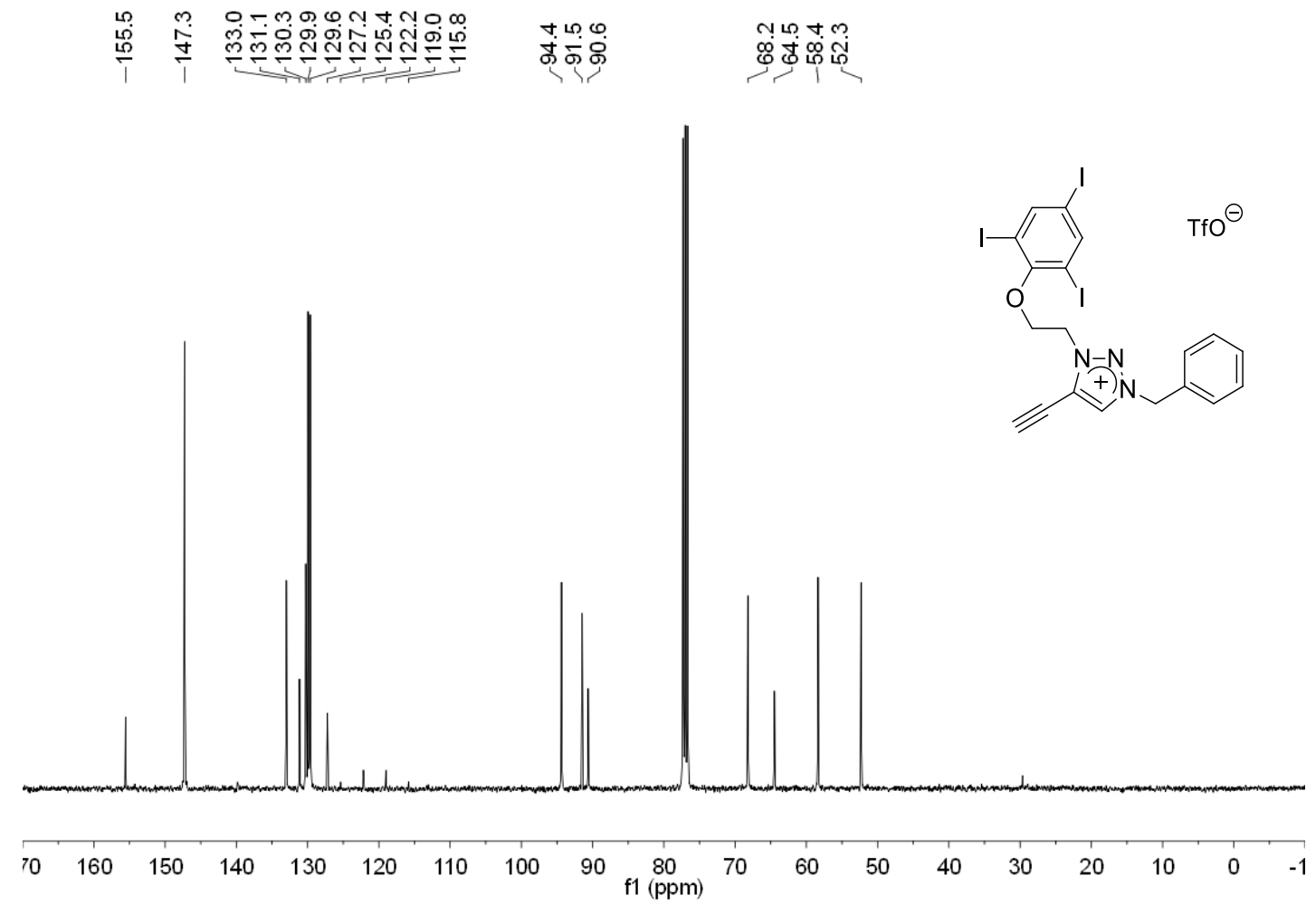

Spectrum 34. ${ }^{13} \mathrm{C}$ NMR $\left(101 \mathrm{MHz}, \mathrm{CDCl}_{3}\right)$ spectrum of compound $2 \mathbf{i}$. 


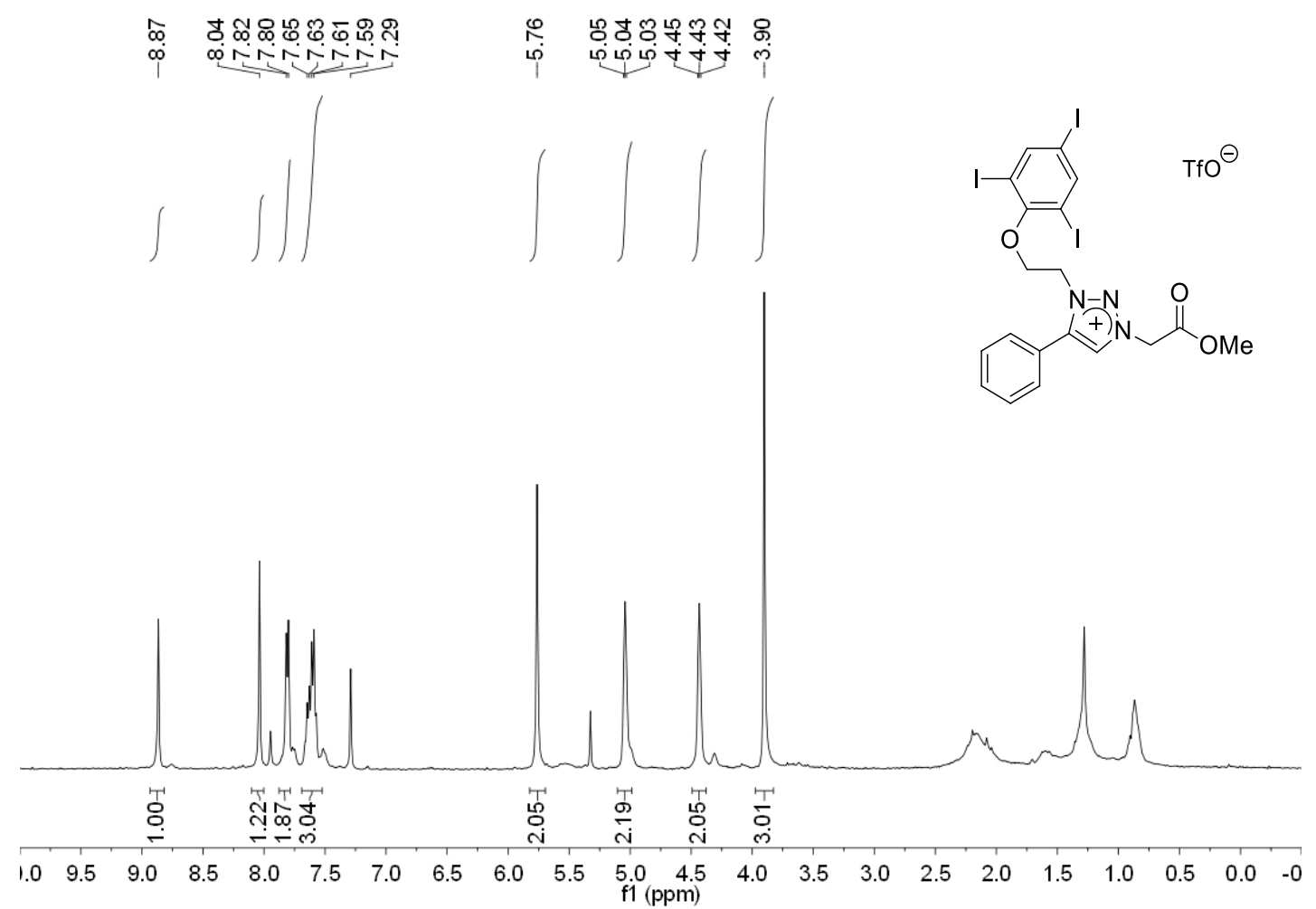

Spectrum 35. ${ }^{1} \mathrm{H}$ NMR ( $\left.400 \mathrm{MHz}, \mathrm{CDCl}_{3}\right)$ spectrum of compound $\mathbf{2 j}$.

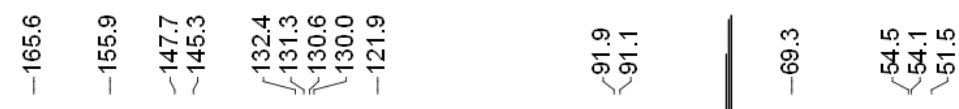

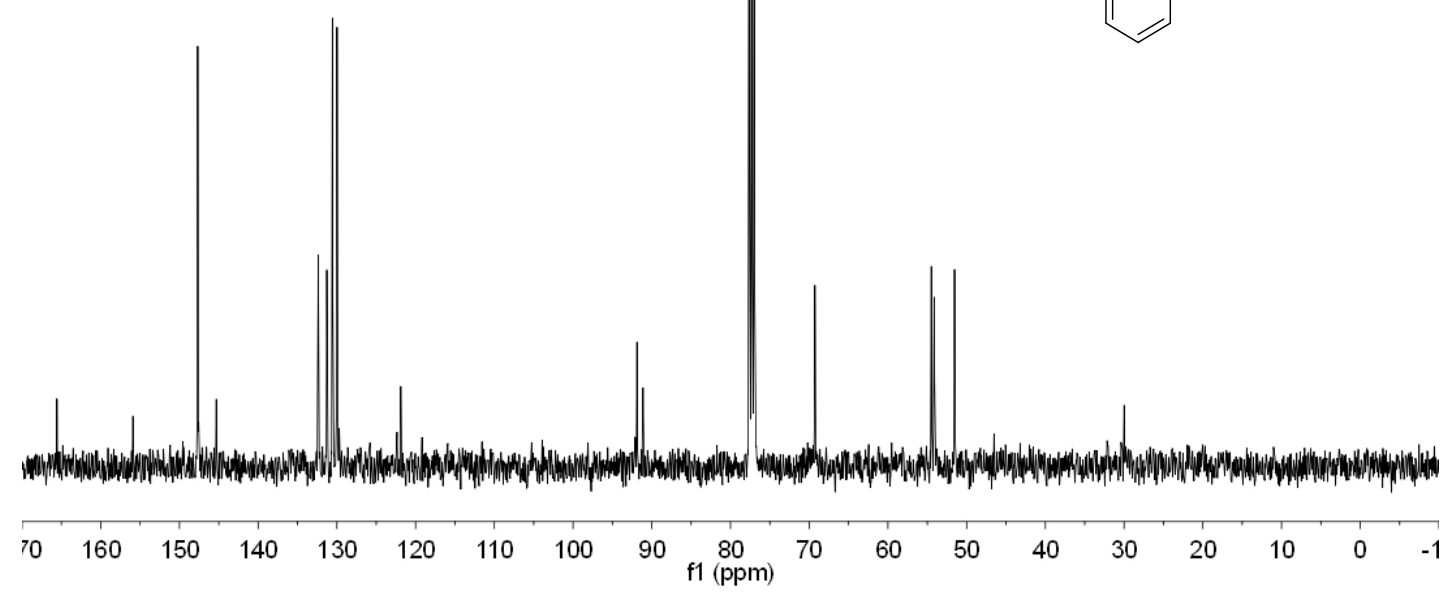

Spectrum 36. ${ }^{13} \mathrm{C}$ NMR $\left(101 \mathrm{MHz}, \mathrm{CDCl}_{3}\right)$ spectrum of compound $\mathbf{2 j}$. 


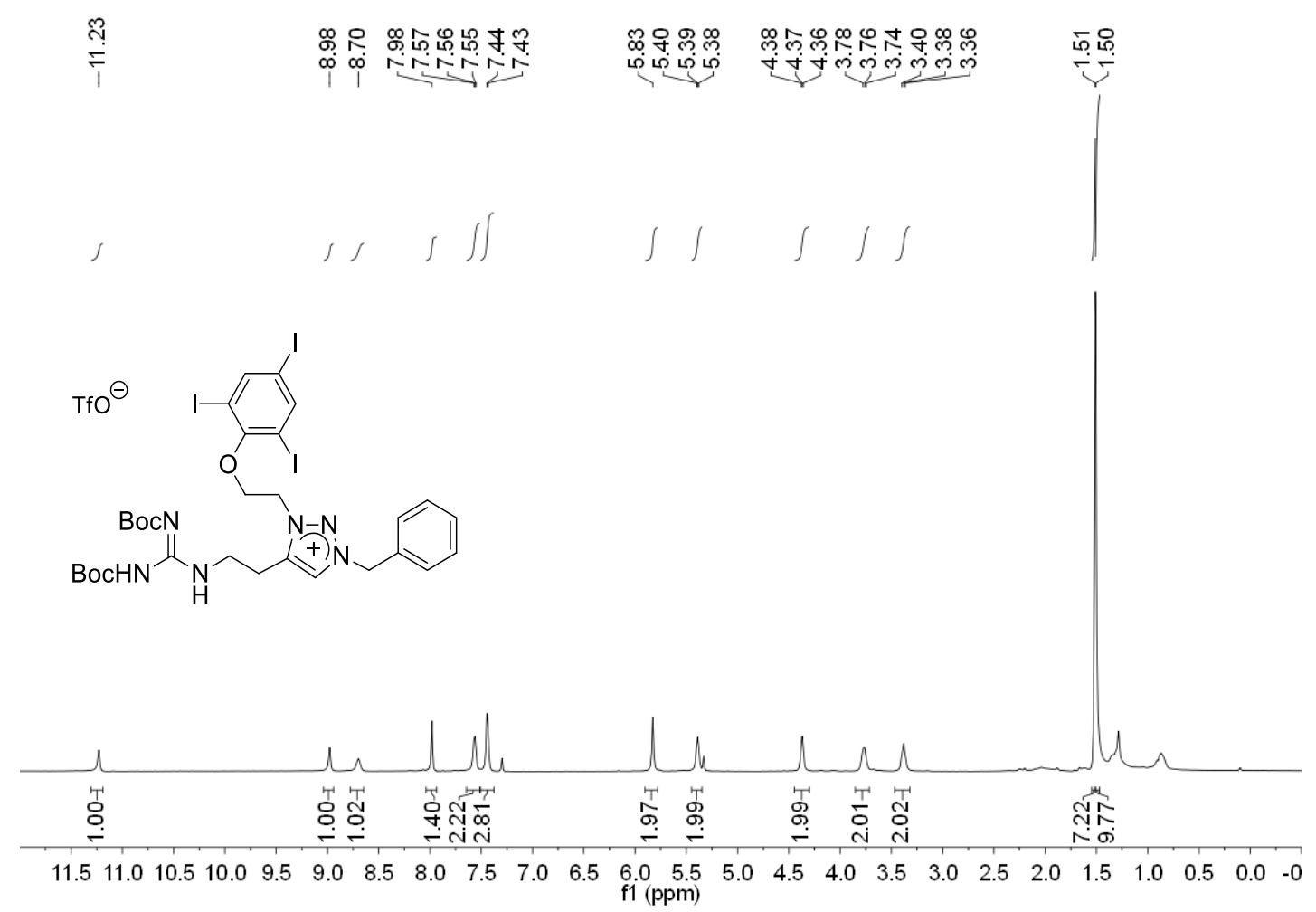

Spectrum 37. ${ }^{1} \mathrm{H}$ NMR $\left(400 \mathrm{MHz}, \mathrm{CDCl}_{3}\right)$ spectrum of compound $\mathbf{2 k}$.
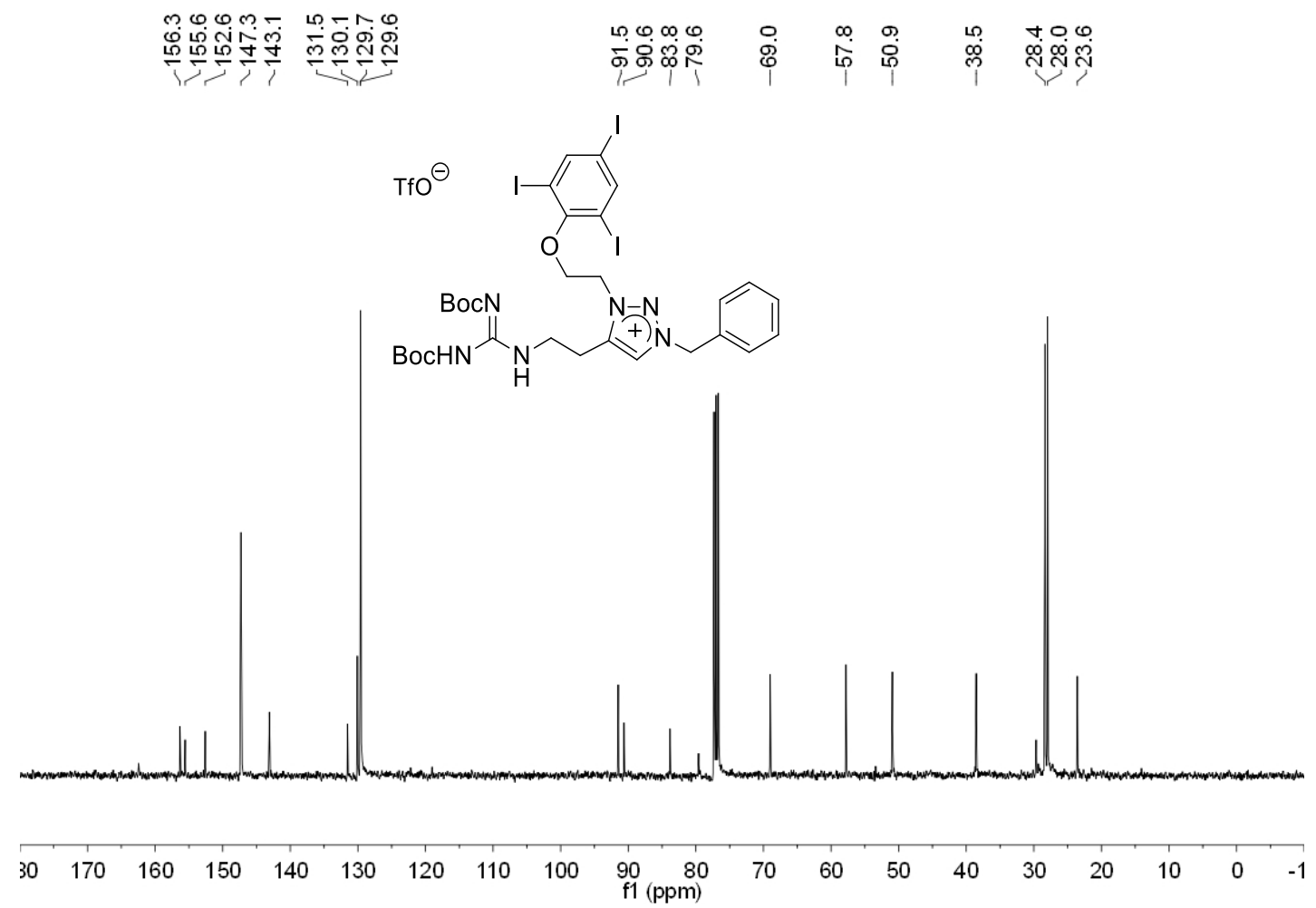

Spectrum 38. ${ }^{13} \mathrm{C}$ NMR $\left(101 \mathrm{MHz}, \mathrm{CDCl}_{3}\right)$ spectrum of compound $\mathbf{2 k}$. 


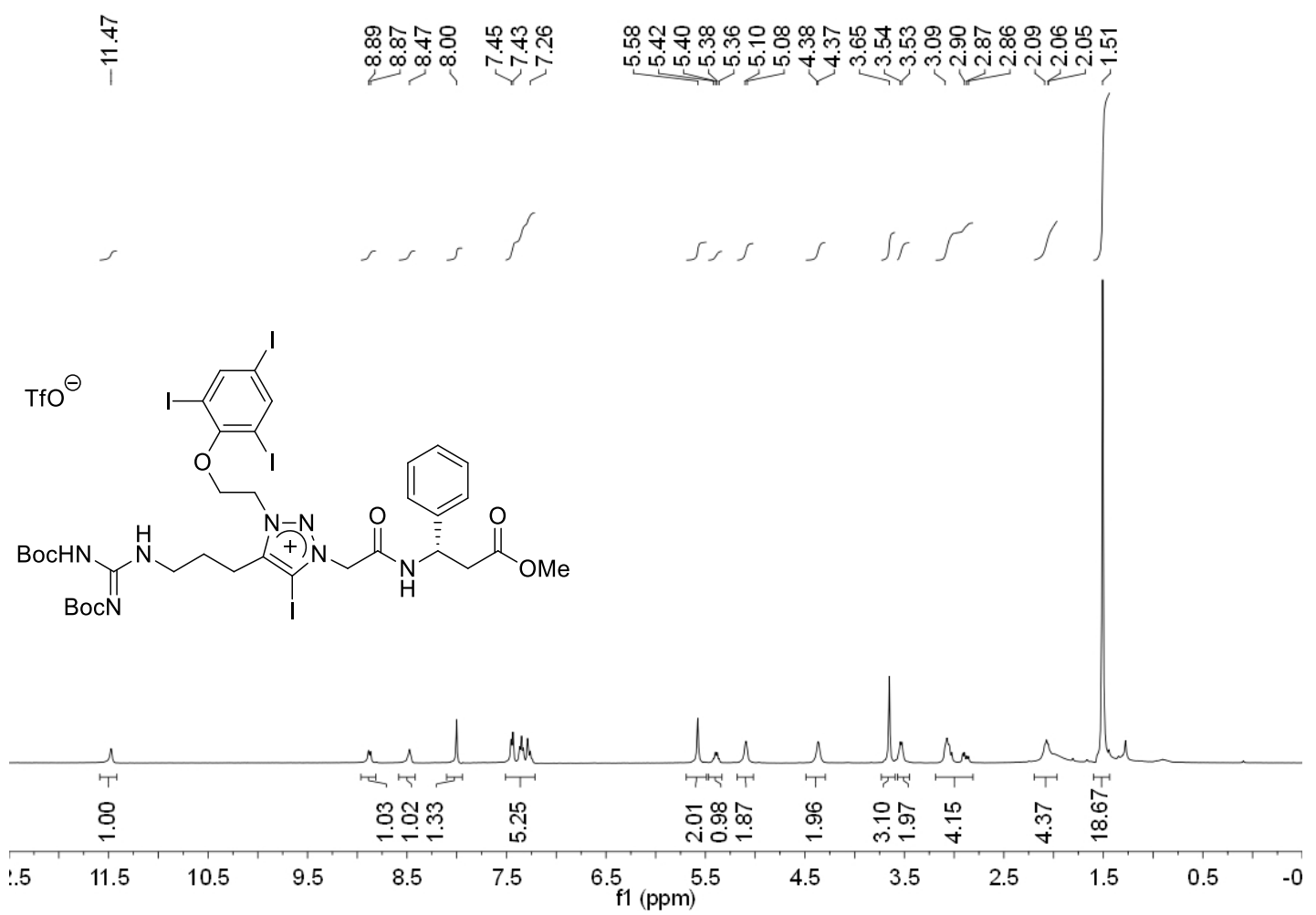

Spectrum 39. ${ }^{1} \mathrm{H}$ NMR (400 MHz, $\mathrm{CDCl}_{3}$ ) spectrum of compound $2 \mathbf{2}$.

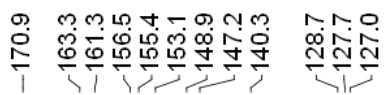
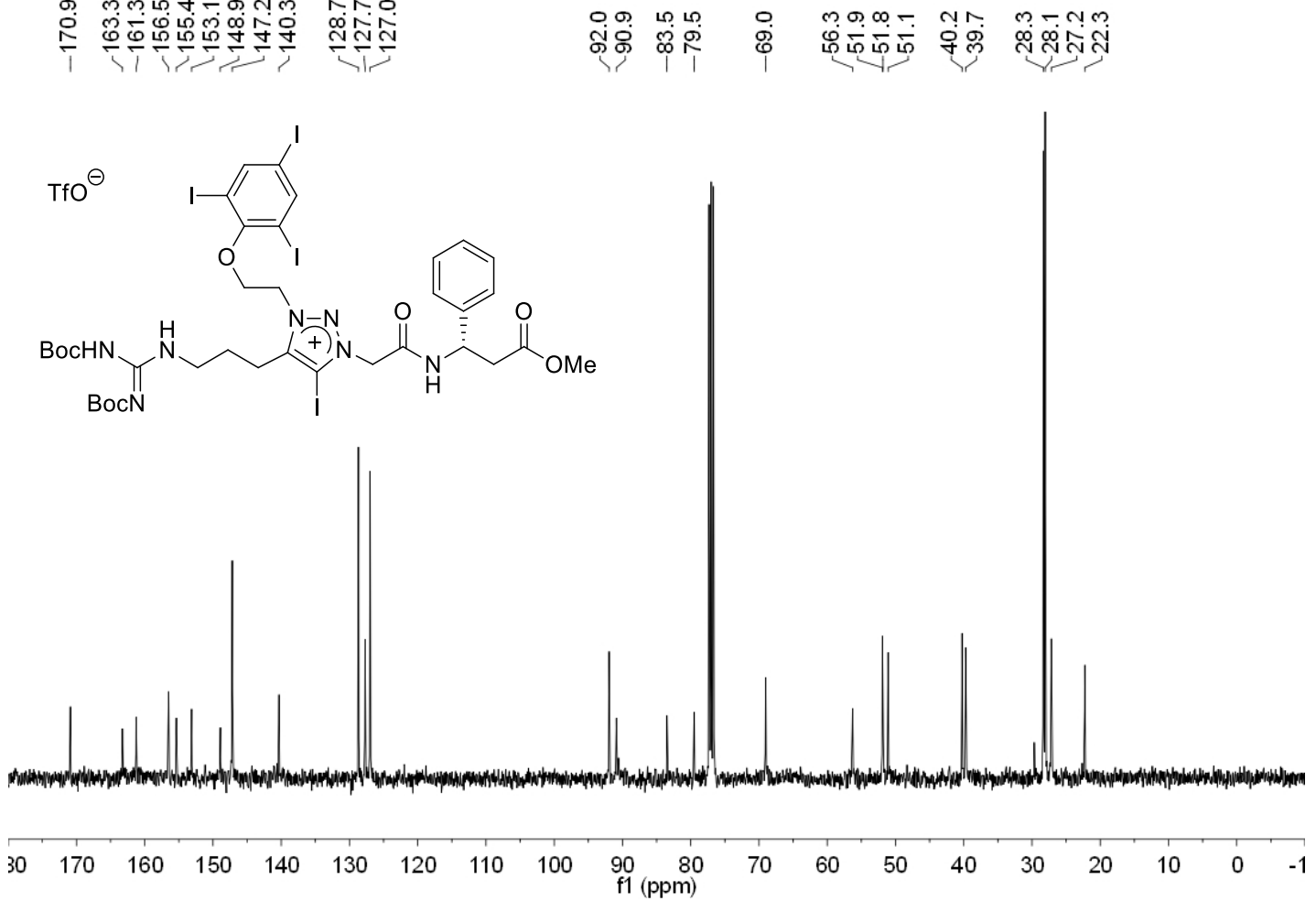

Spectrum 40. ${ }^{13} \mathrm{C}$ NMR $\left(101 \mathrm{MHz}, \mathrm{CDCl}_{3}\right)$ spectrum of compound 21. 


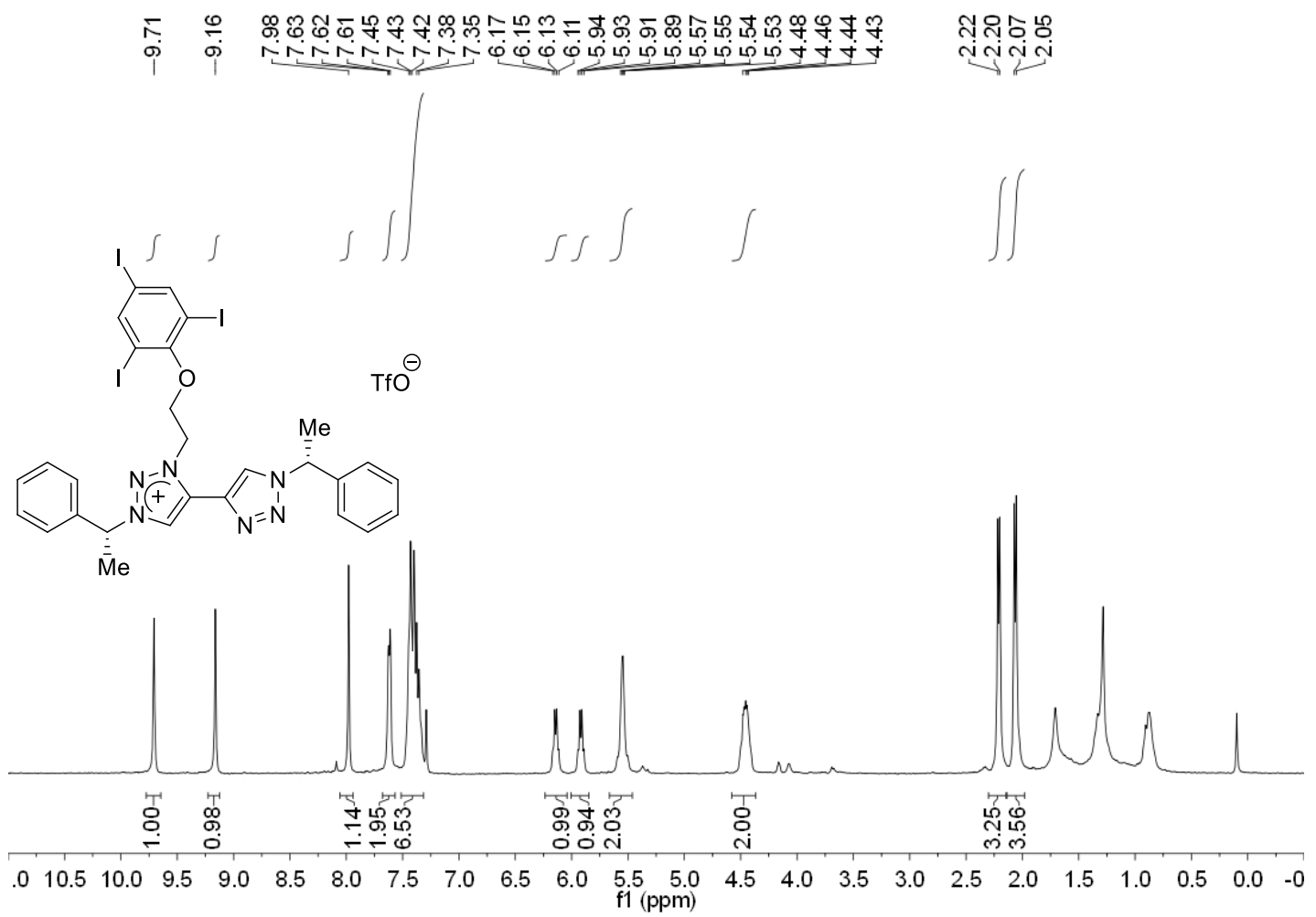

Spectrum 41. ${ }^{1} \mathrm{H}$ NMR $\left(400 \mathrm{MHz}, \mathrm{CDCl}_{3}\right)$ spectrum of compound $\mathbf{2 m}$.

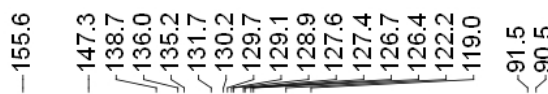

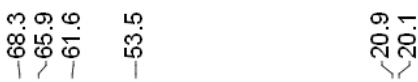

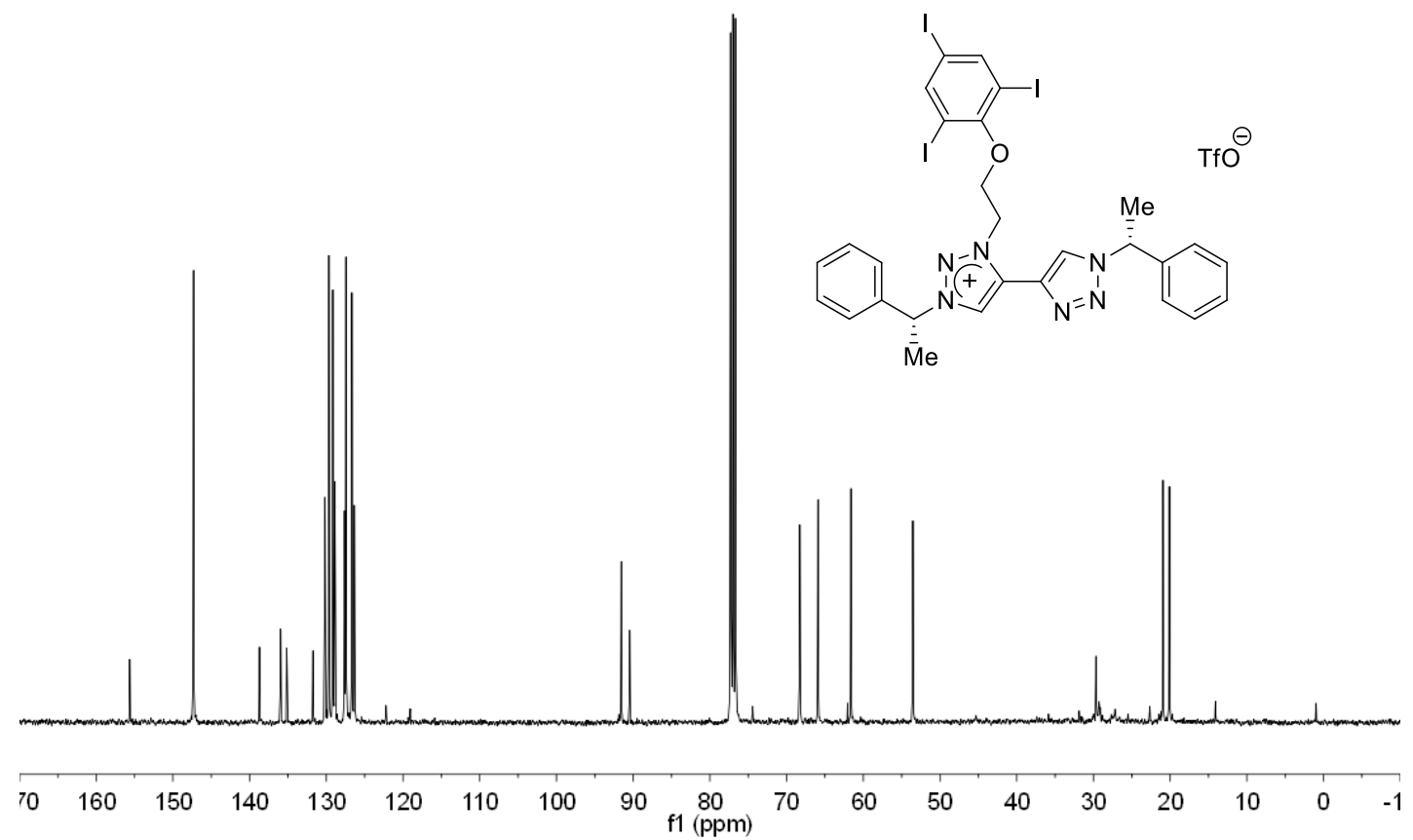

Spectrum 42. ${ }^{13} \mathrm{C}$ NMR $\left(101 \mathrm{MHz}, \mathrm{CDCl}_{3}\right)$ spectrum of compound $\mathbf{2 m}$. 


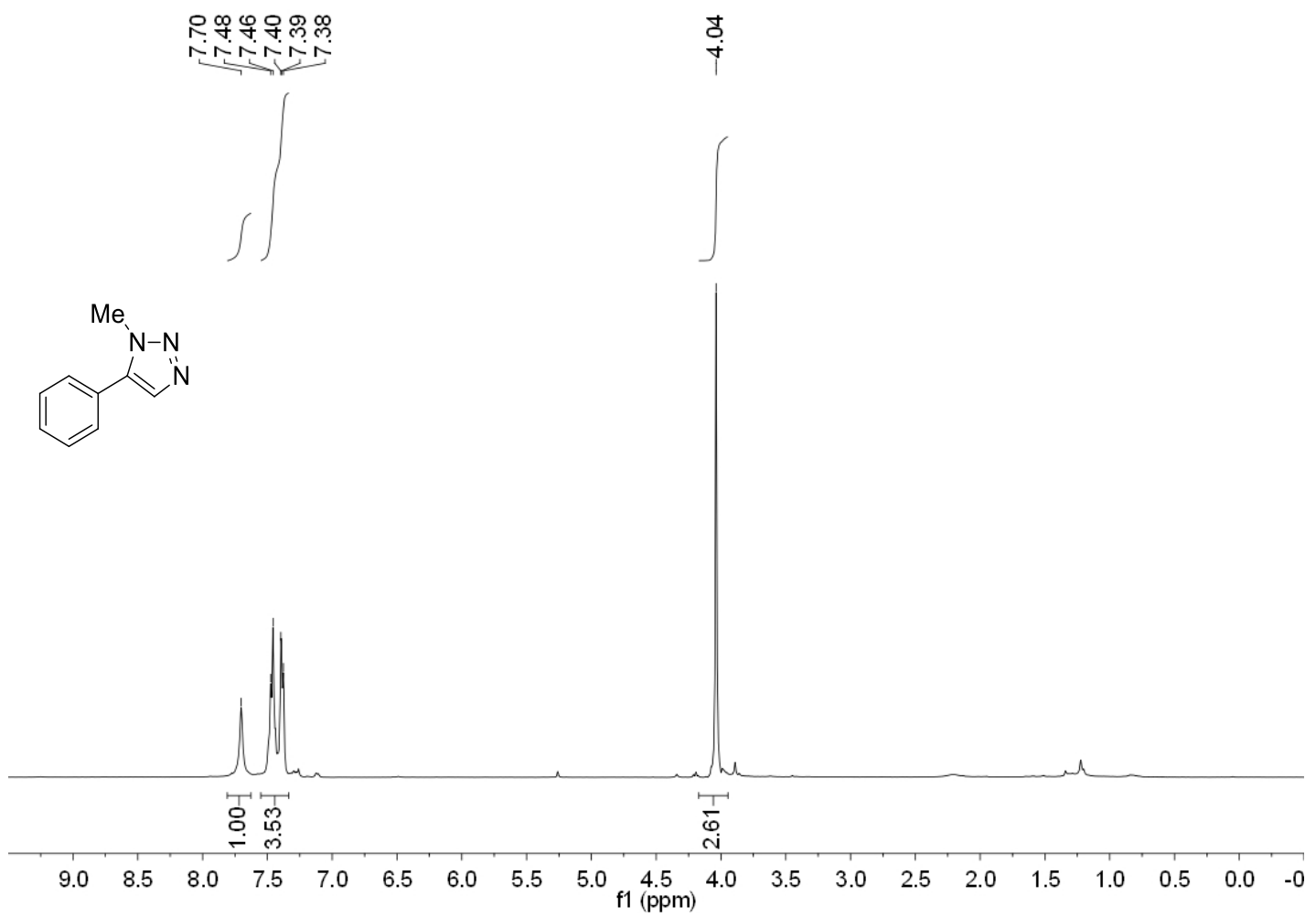

Spectrum 43. ${ }^{1} \mathrm{H}$ NMR $\left(400 \mathrm{MHz}, \mathrm{CDCl}_{3}\right)$ spectrum of compound $\mathbf{6 a}$.

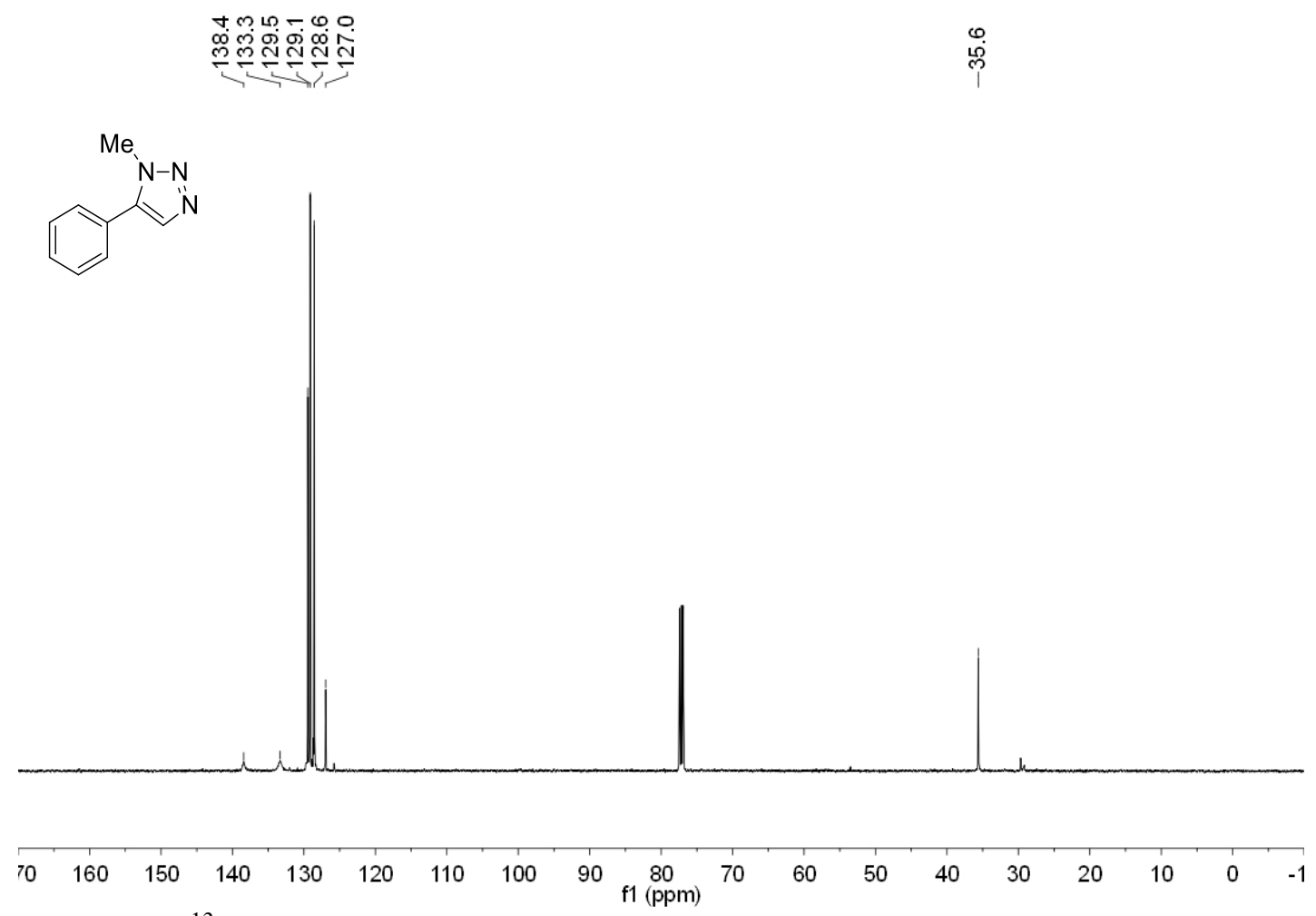

Spectrum 44. ${ }^{13} \mathrm{C}$ NMR $\left(126 \mathrm{MHz}, \mathrm{CDCl}_{3}\right)$ spectrum of compound $6 \mathbf{a}$. 


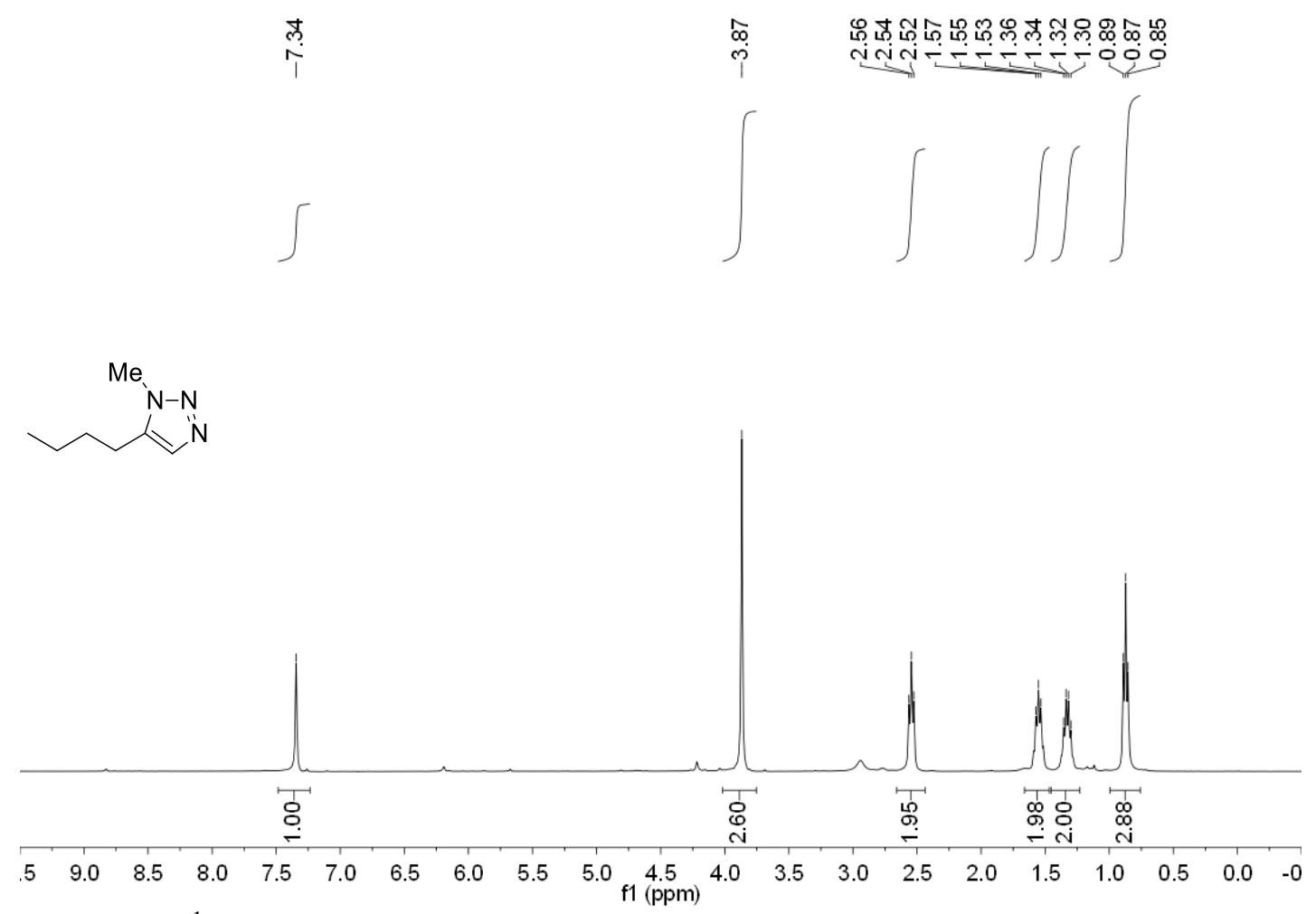

Spectrum 45. ${ }^{1} \mathrm{H}$ NMR $\left(400 \mathrm{MHz}, \mathrm{CDCl}_{3}\right)$ spectrum of compound $\mathbf{6 b}$.

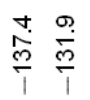

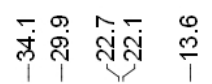

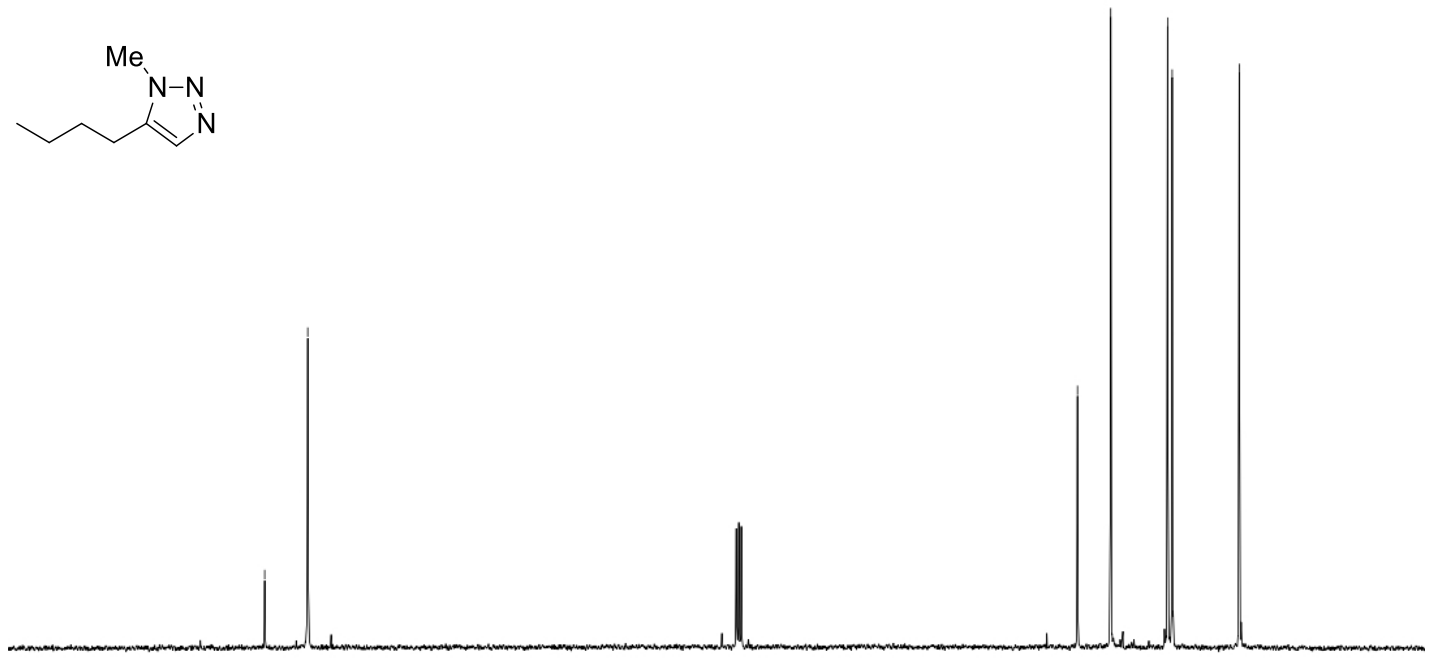

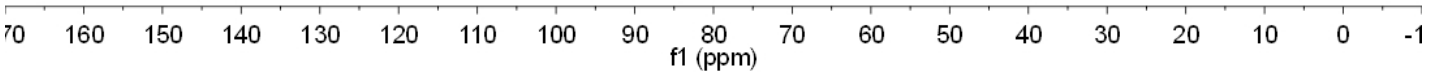

Spectrum 46. ${ }^{13} \mathrm{C}$ NMR $\left(101 \mathrm{MHz}, \mathrm{CDCl}_{3}\right)$ spectrum of compound $\mathbf{6 b}$. 

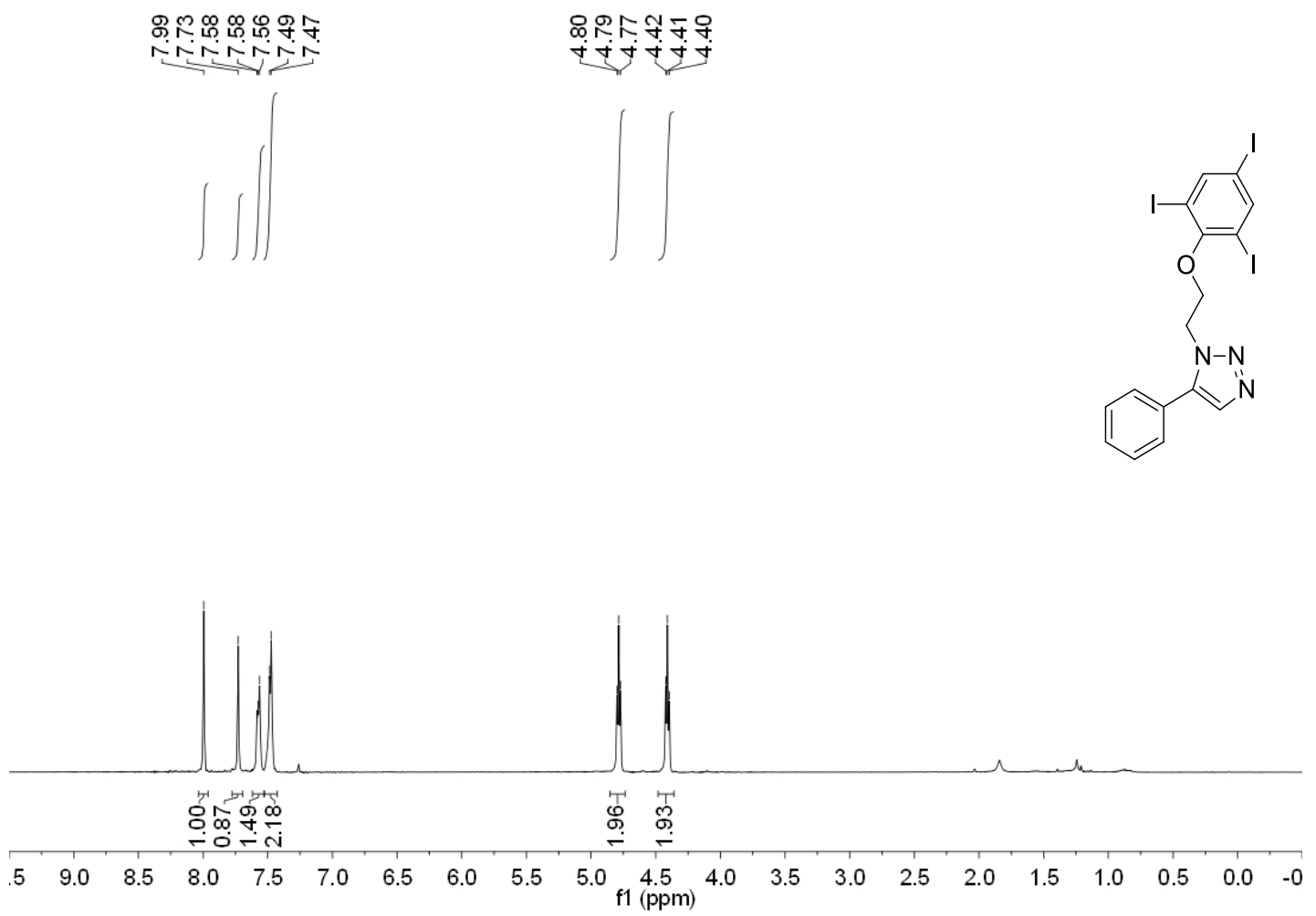

Spectrum 47. ${ }^{1} \mathrm{H}$ NMR $\left(400 \mathrm{MHz}, \mathrm{CDCl}_{3}\right)$ spectrum of compound $\mathbf{6 c}$.
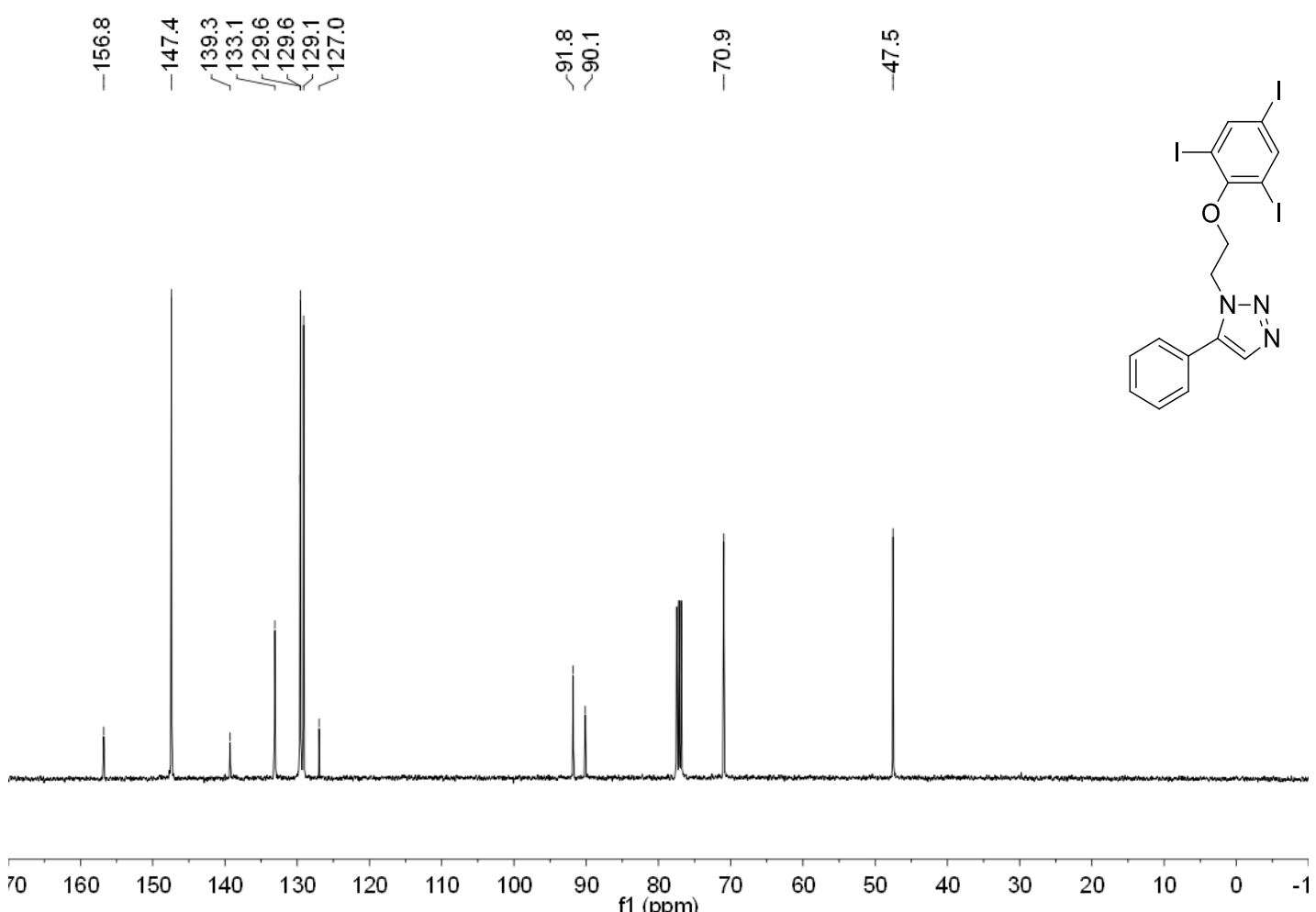

Spectrum 48. ${ }^{13} \mathrm{C}$ NMR (101 MHz, $\left.\mathrm{CDCl}_{3}\right)$ spectrum of compound $\mathbf{6 c}$. 


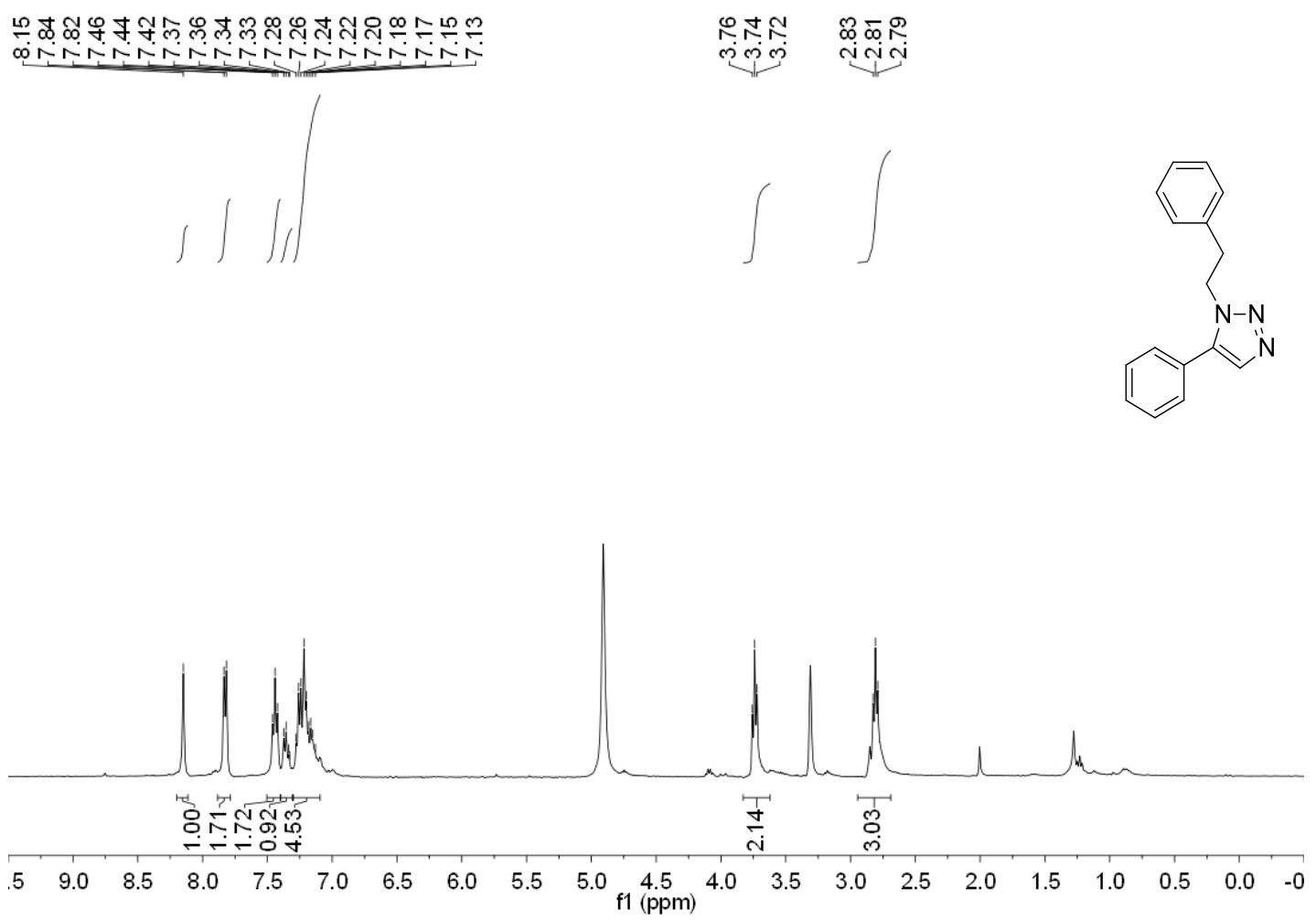

Spectrum 49. ${ }^{1} \mathrm{H}$ NMR (400 MHz, MeOH-d ${ }_{4}$ ) spectrum of compound $\mathbf{6 d}$.

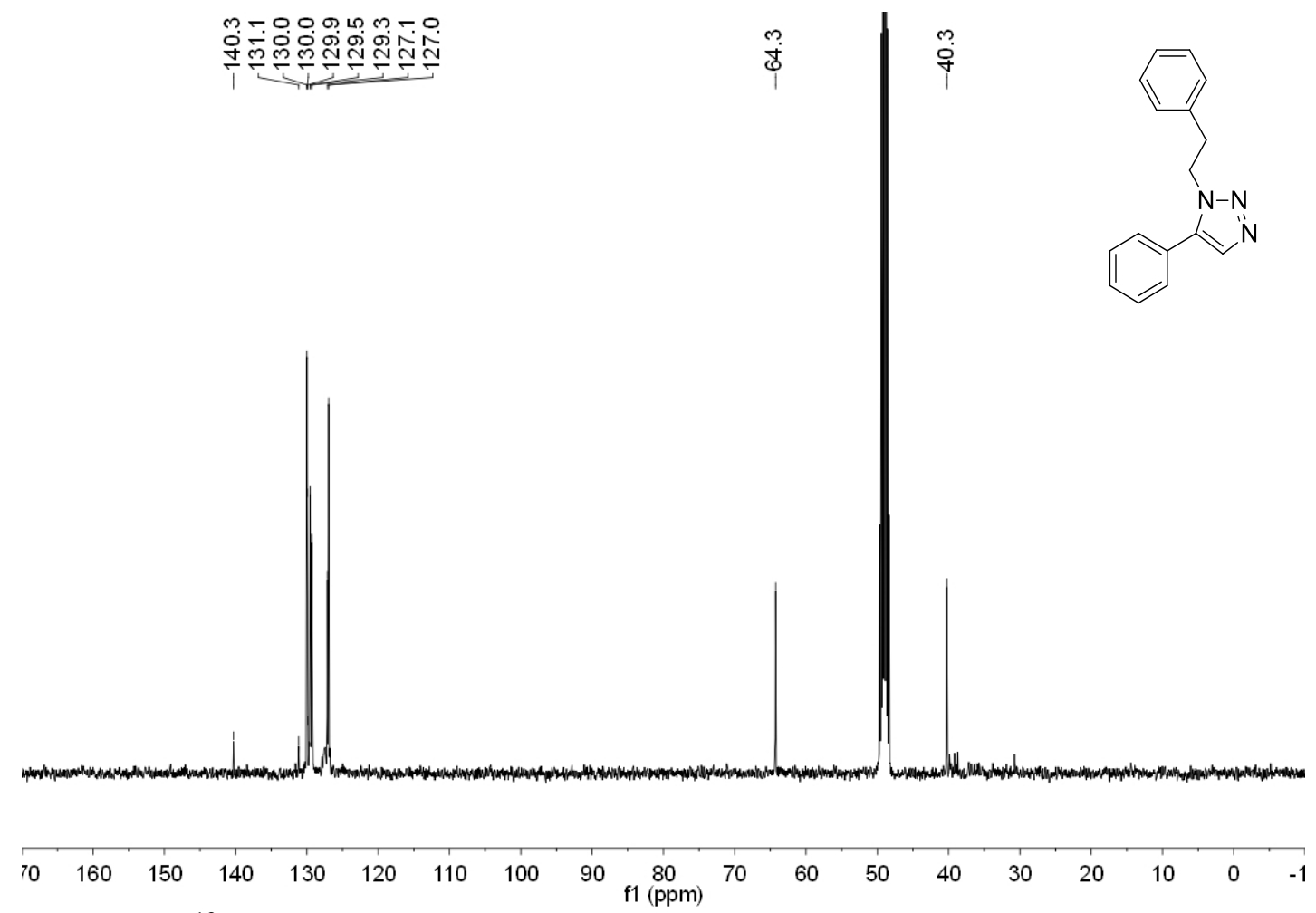

Spectrum 50. ${ }^{13} \mathrm{C}$ NMR (101 MHz, MeOH-d 4 ) spectrum of compound $\mathbf{6 d}$. 

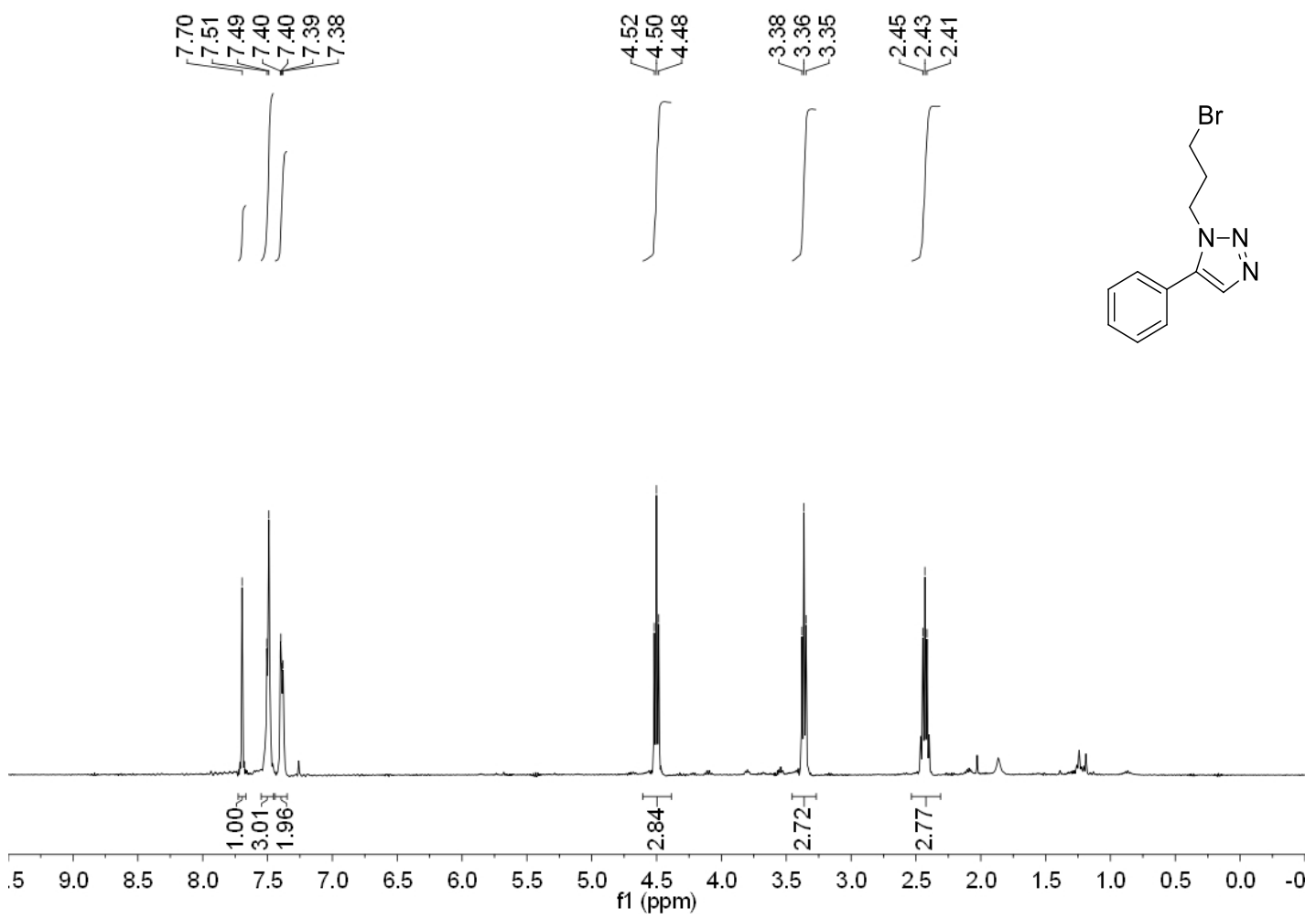

Spectrum 51. ${ }^{1} \mathrm{H}$ NMR (400 $\left.\mathrm{MHz}, \mathrm{CDCl}_{3}\right)$ spectrum of compound $\mathbf{6 e}$.
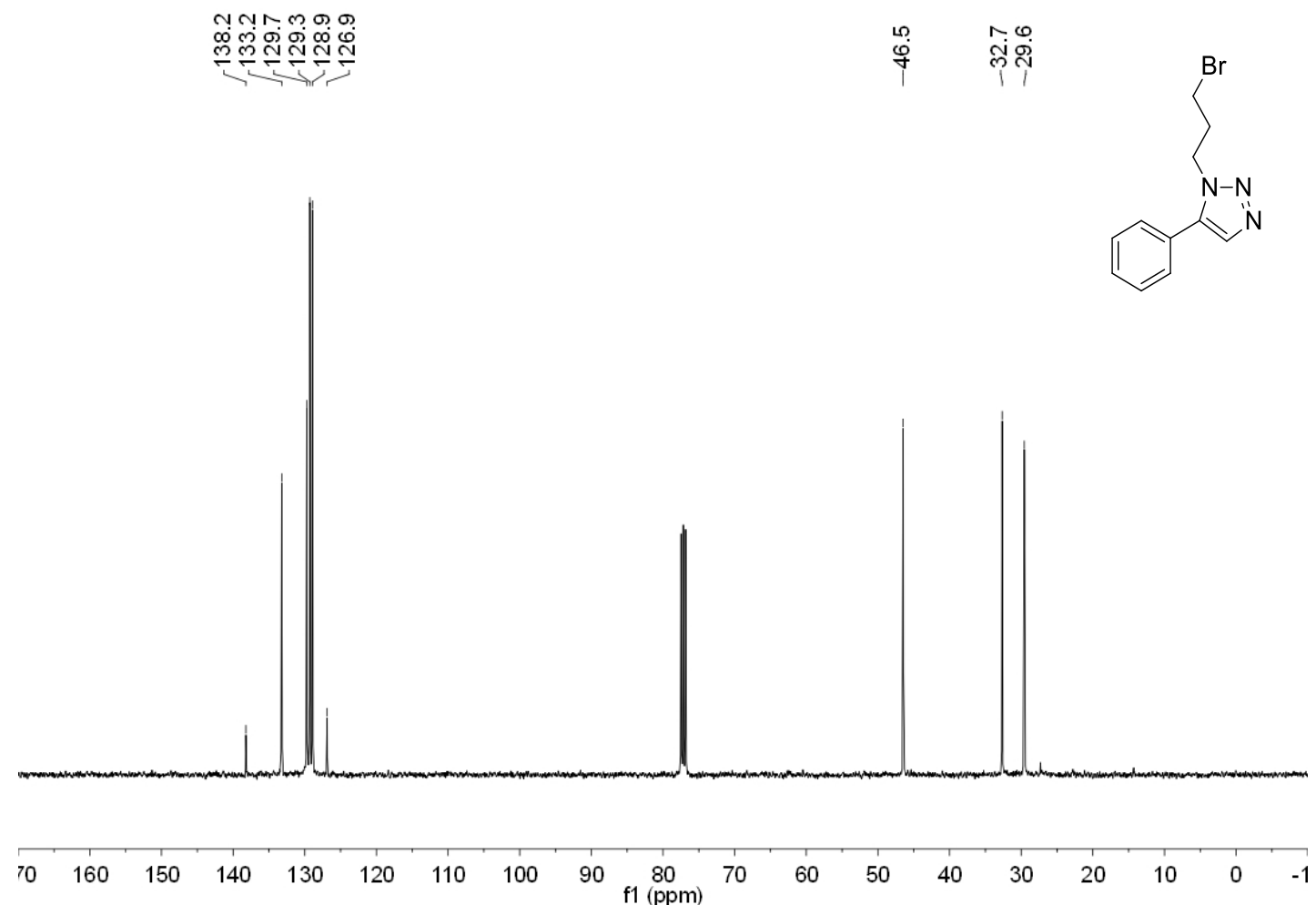

Spectrum 52. ${ }^{13} \mathrm{C}$ NMR $\left(101 \mathrm{MHz}, \mathrm{CDCl}_{3}\right)$ spectrum of compound $\mathbf{6 e}$. 

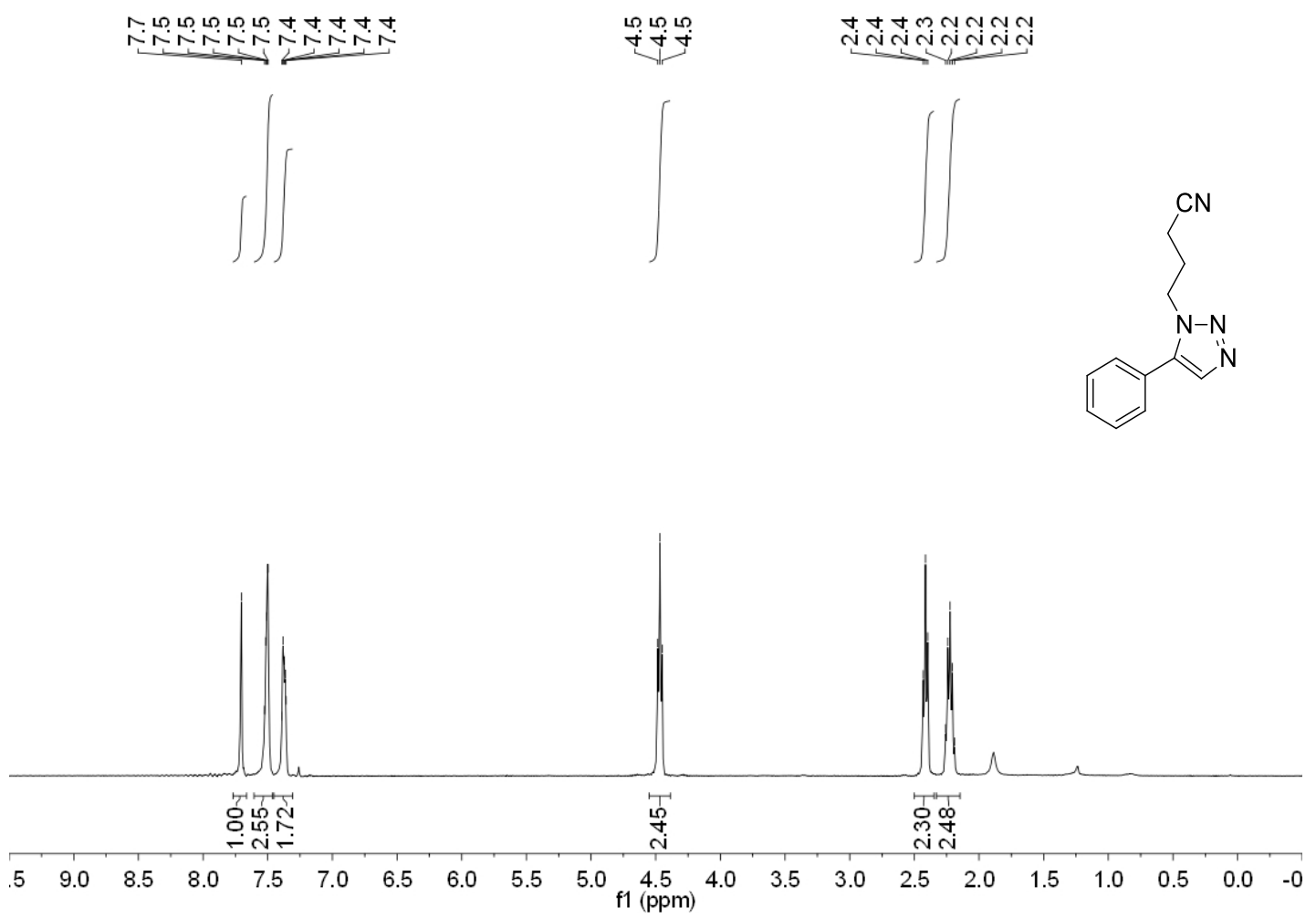

Spectrum 53. ${ }^{1} \mathrm{H}$ NMR $\left(400 \mathrm{MHz}, \mathrm{CDCl}_{3}\right)$ spectrum of compound $\mathbf{6 f}$.
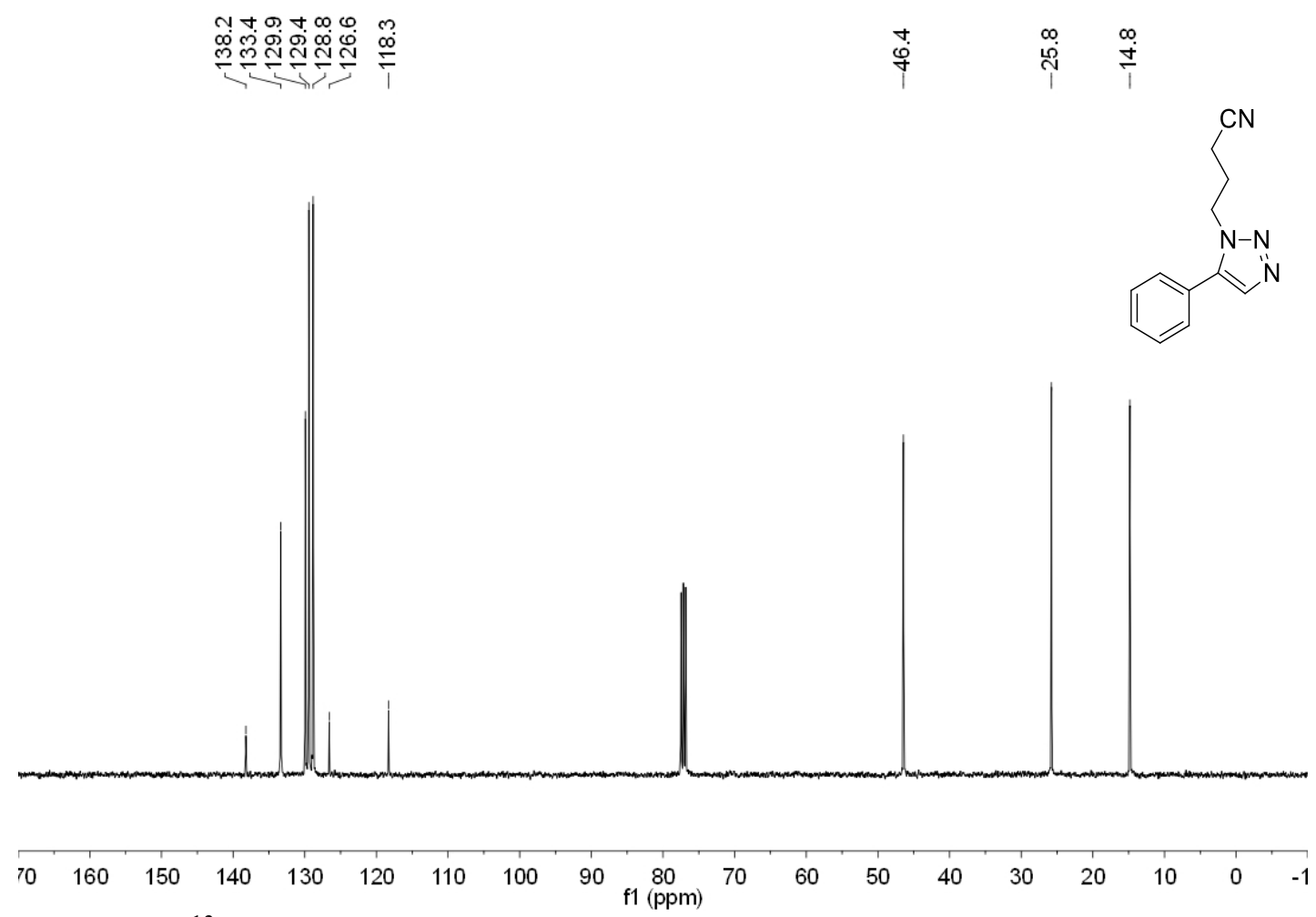

Spectrum 54. ${ }^{13} \mathrm{C}$ NMR (101 MHz, $\left.\mathrm{CDCl}_{3}\right)$ spectrum of compound $\mathbf{6 f}$. 


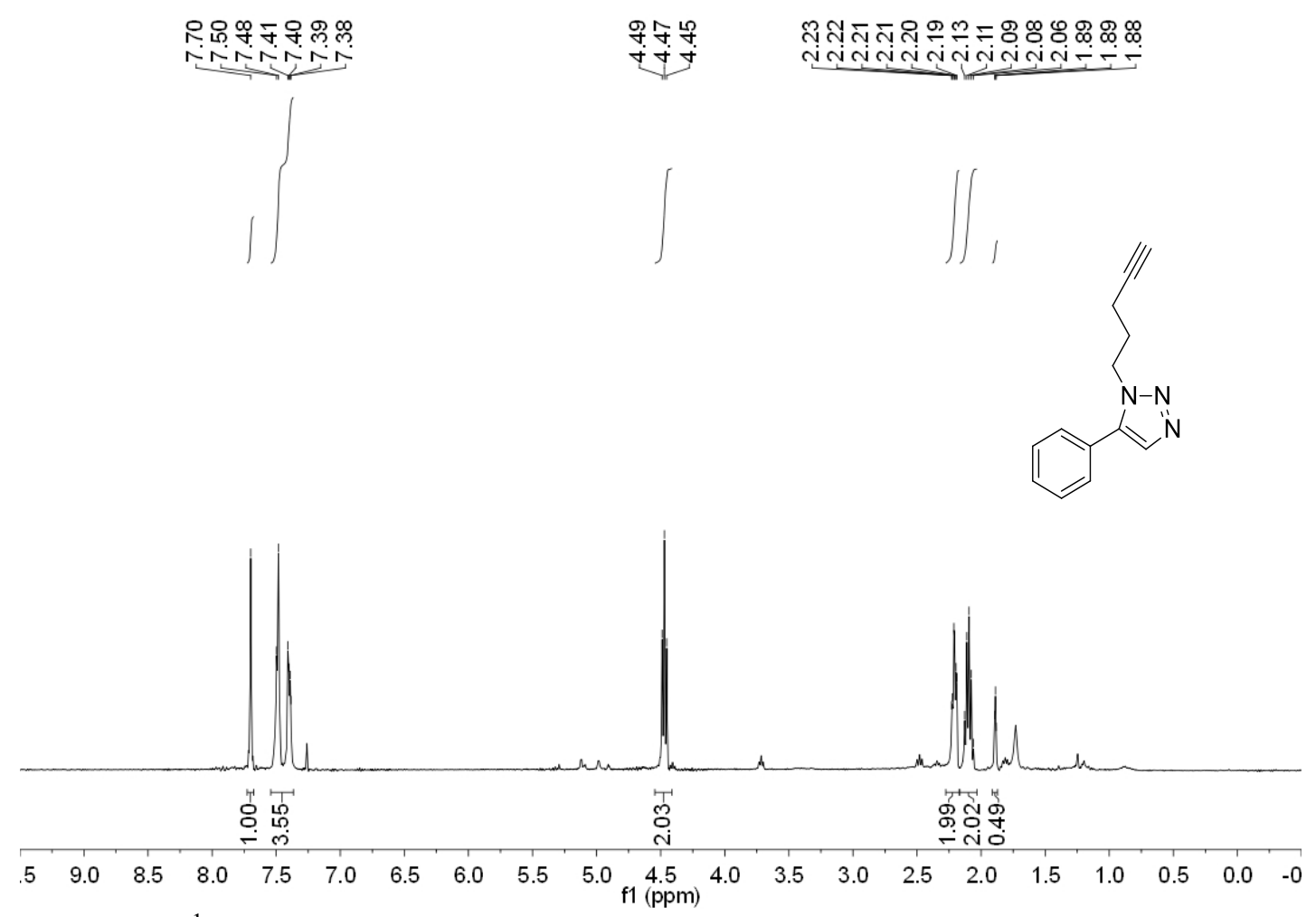

Spectrum 55. ${ }^{1} \mathrm{H}$ NMR $\left(400 \mathrm{MHz}, \mathrm{CDCl}_{3}\right)$ spectrum of compound $\mathbf{6 g}$.

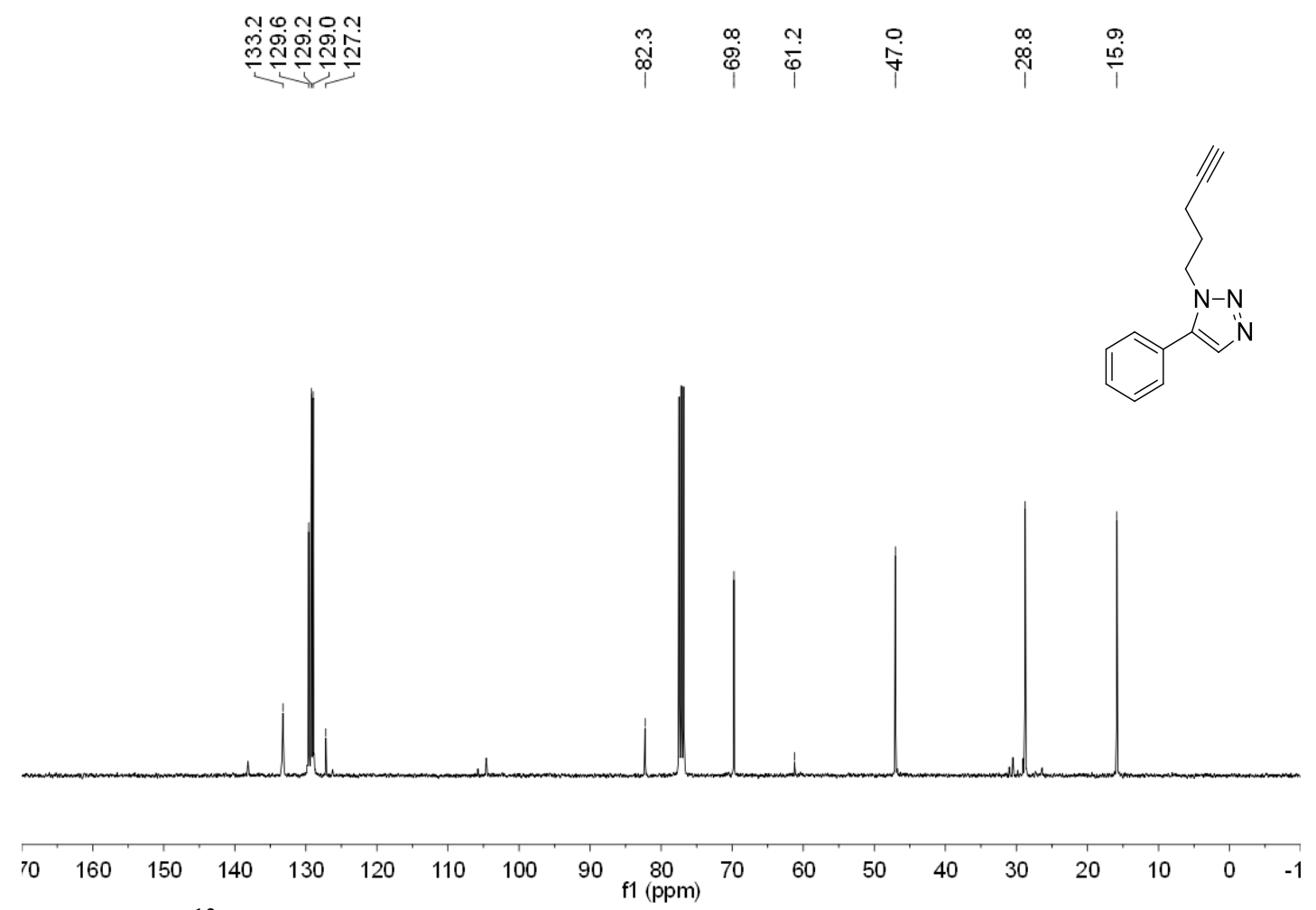

Spectrum 56. ${ }^{13} \mathrm{C}$ NMR $\left(101 \mathrm{MHz}, \mathrm{CDCl}_{3}\right)$ spectrum of compound $\mathbf{6 g}$. 


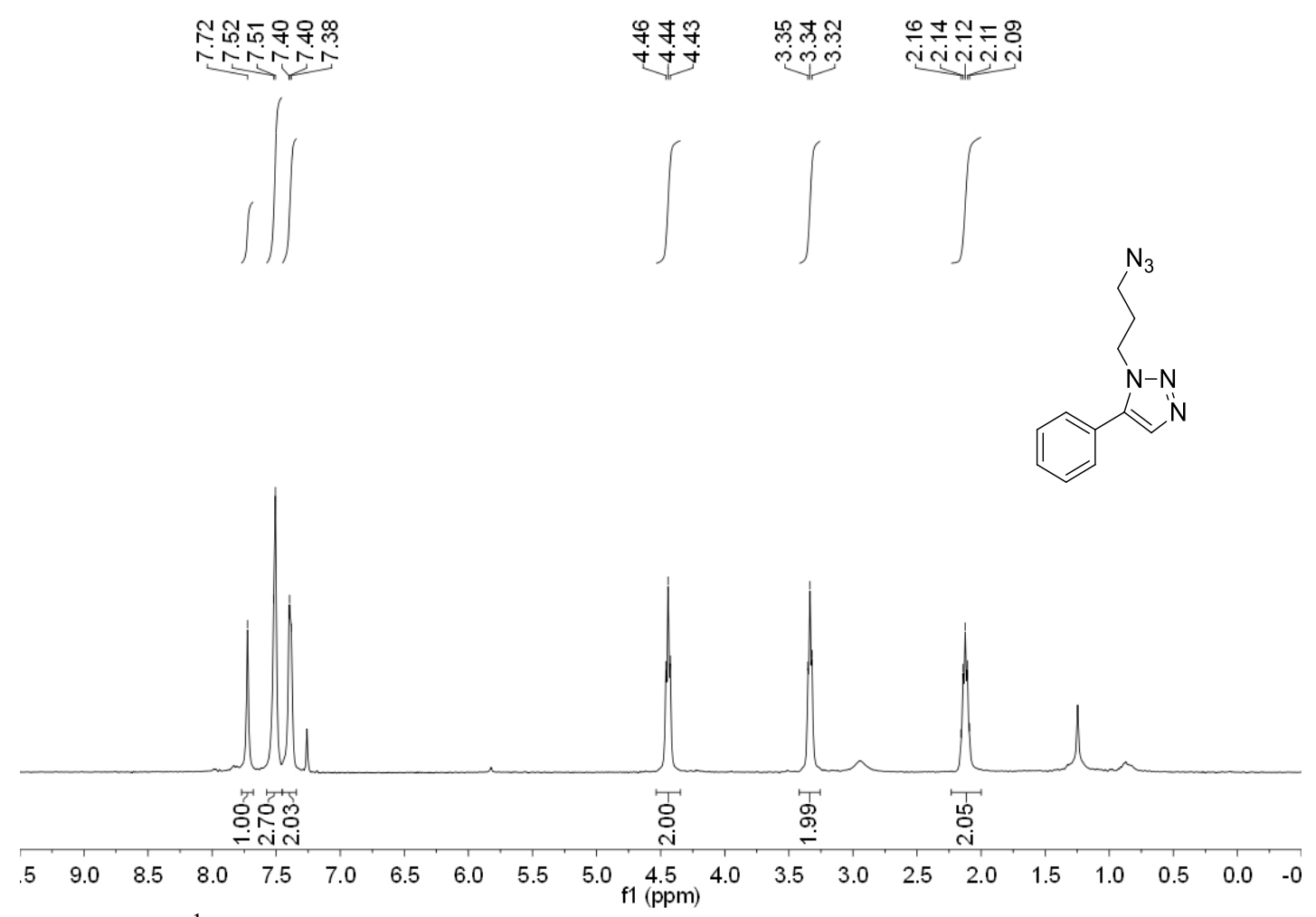

Spectrum 57. ${ }^{1} \mathrm{H}$ NMR $\left(400 \mathrm{MHz}, \mathrm{CDCl}_{3}\right)$ spectrum of compound $\mathbf{6 h}$.

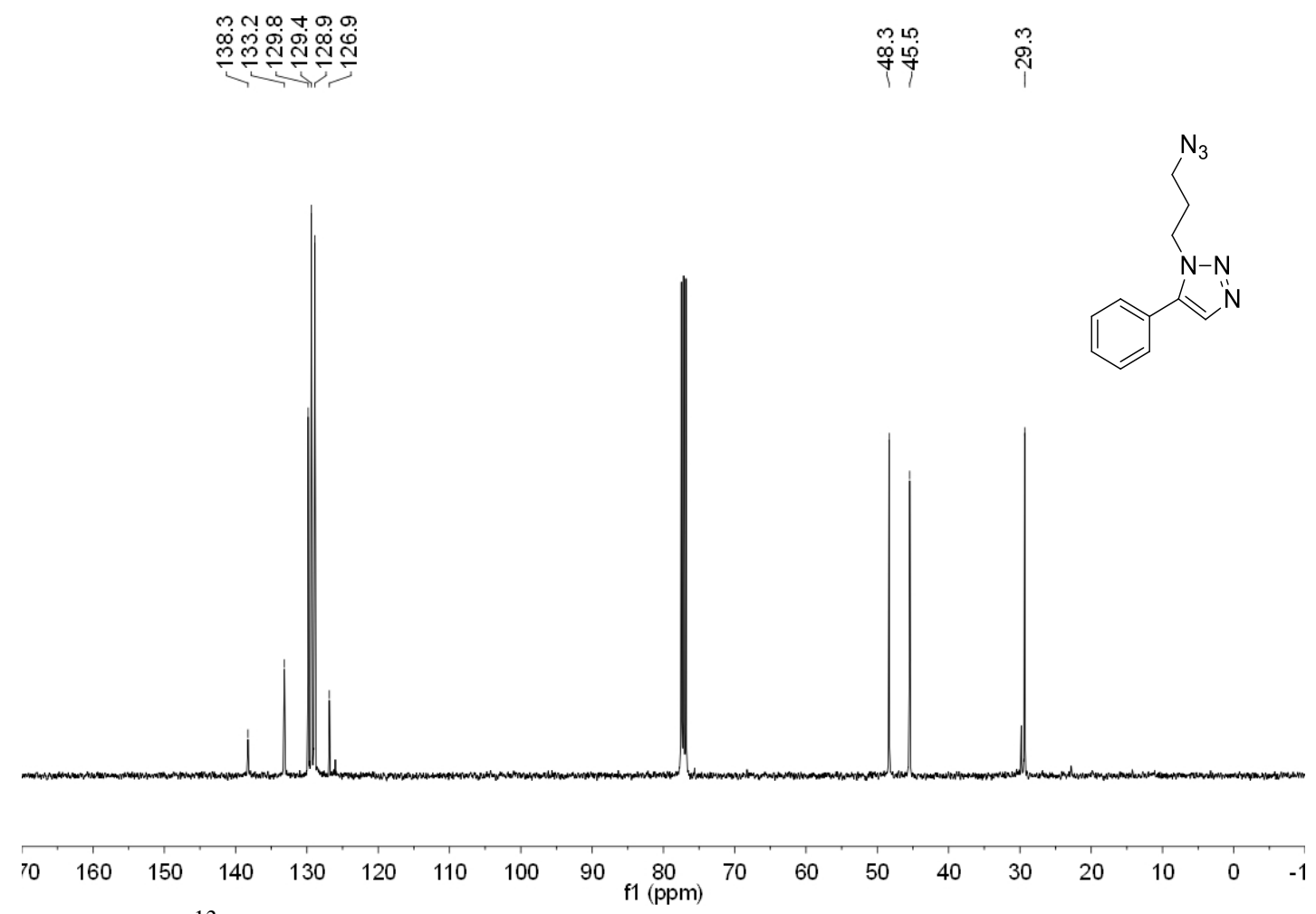

Spectrum 58. ${ }^{13} \mathrm{C}$ NMR (101 MHz, $\left.\mathrm{CDCl}_{3}\right)$ spectrum of compound $\mathbf{6 h}$. 


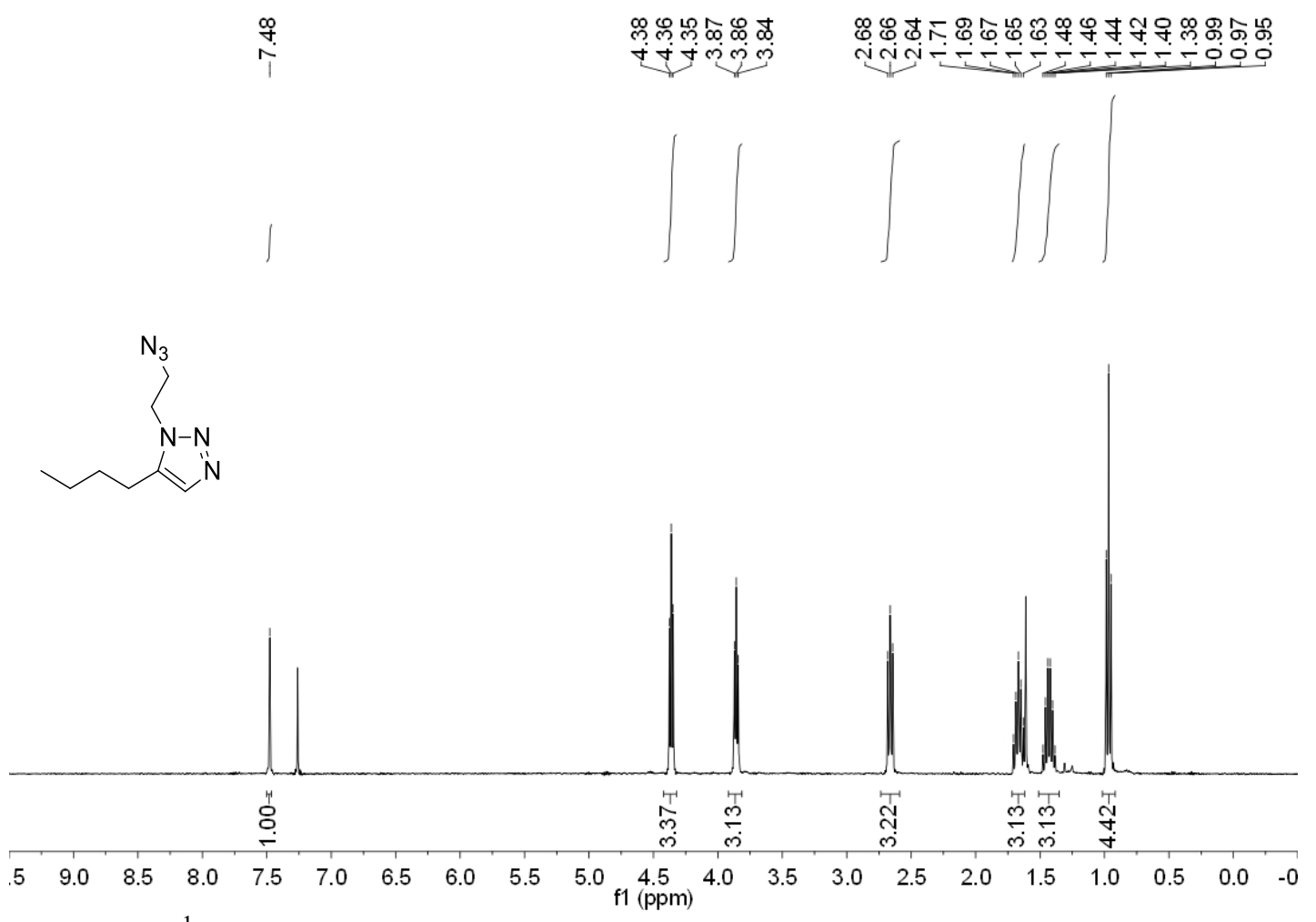

Spectrum 59. ${ }^{1} \mathrm{H}$ NMR (400 MHz, $\mathrm{CDCl}_{3}$ ) spectrum of compound $6 \mathbf{i}$.

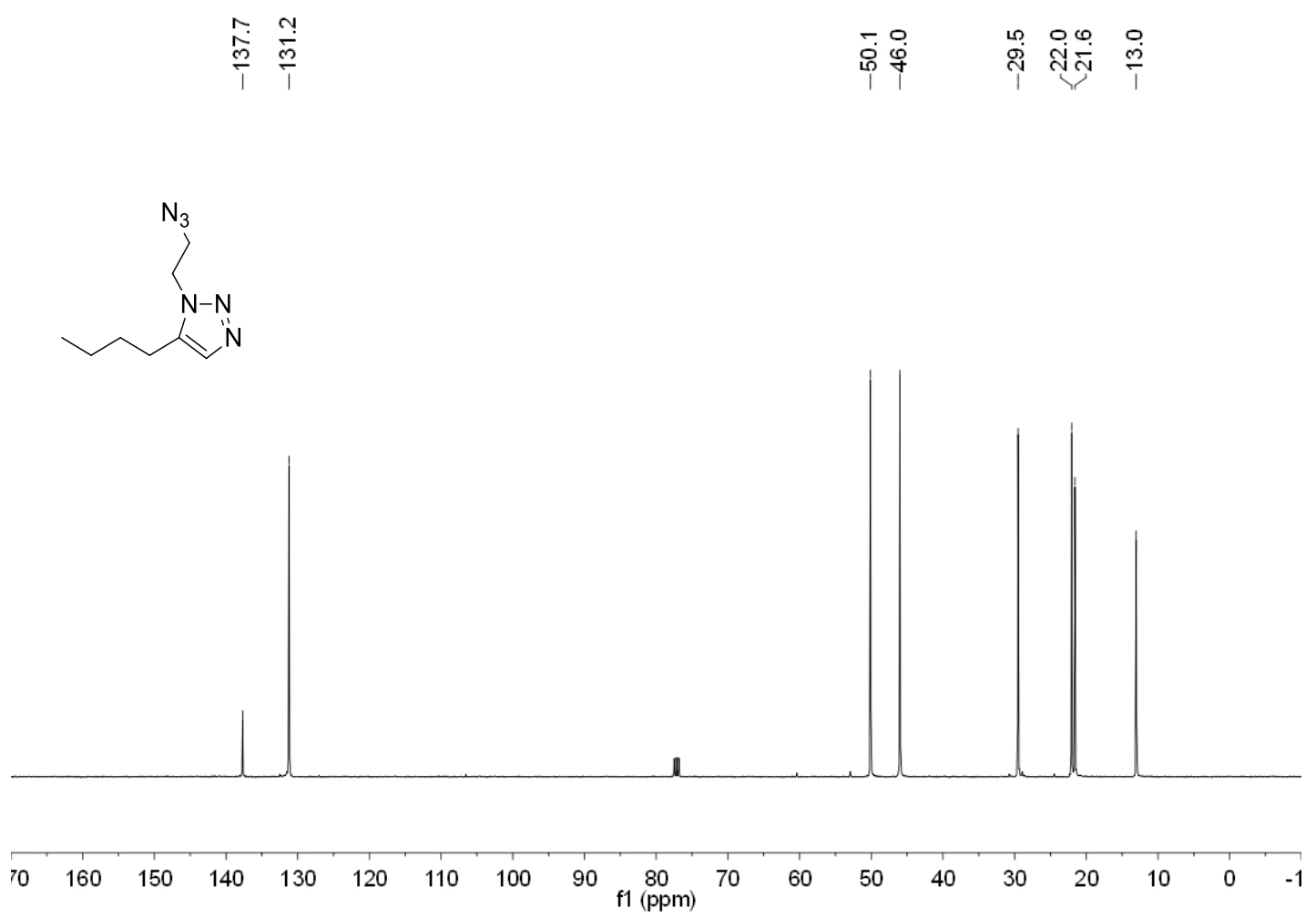

Spectrum 60. ${ }^{13} \mathrm{C}$ NMR $\left(101 \mathrm{MHz}, \mathrm{CDCl}_{3}\right)$ spectrum of compound $6 \mathbf{i}$. 


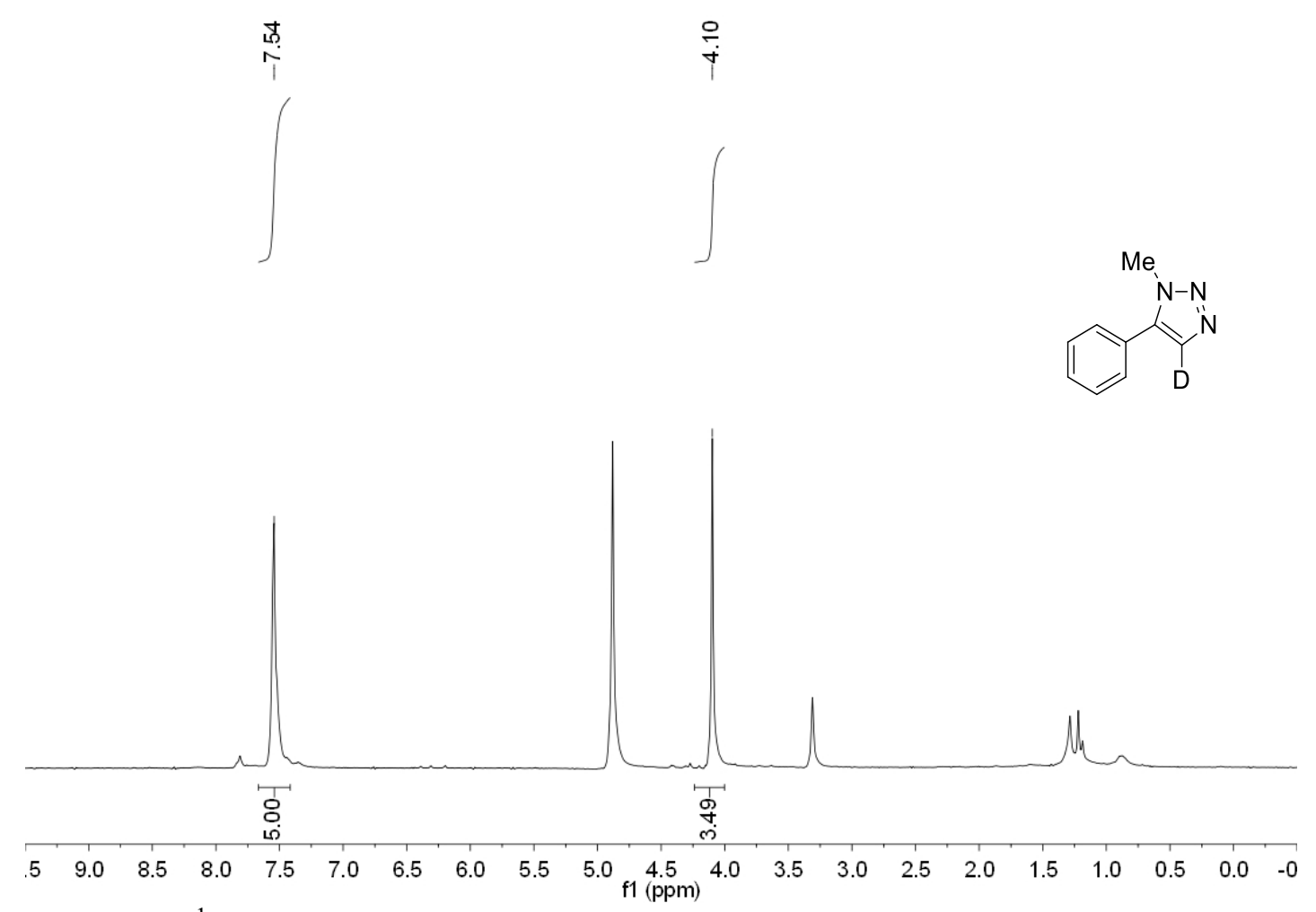

Spectrum 61. ${ }^{1} \mathrm{H}$ NMR (400 MHz, $\mathrm{CDCl}_{3}$ ) spectrum of compound 7.

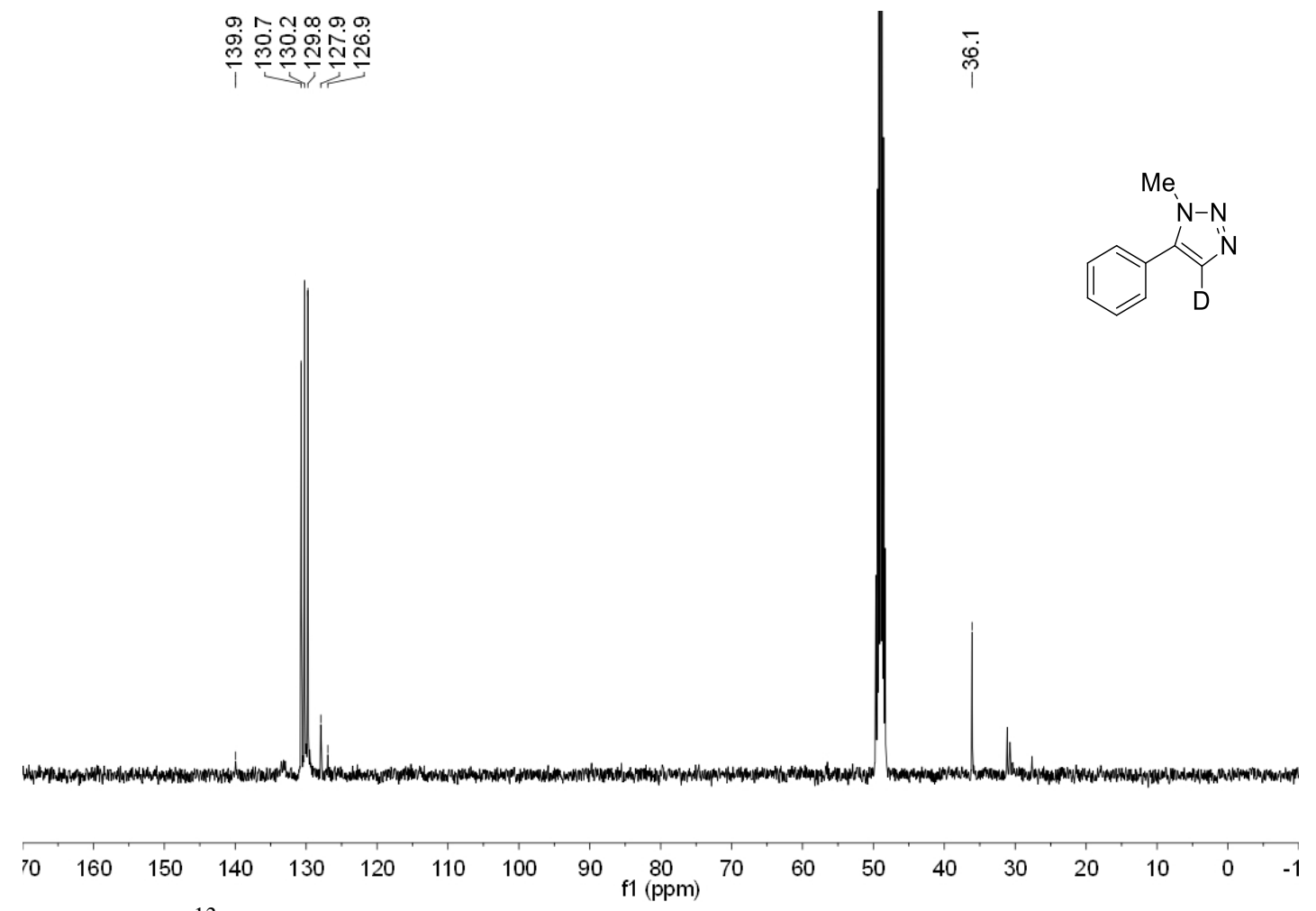

Spectrum 62. ${ }^{13} \mathrm{C}$ NMR $\left(101 \mathrm{MHz}, \mathrm{CDCl}_{3}\right)$ spectrum of compound 7. 


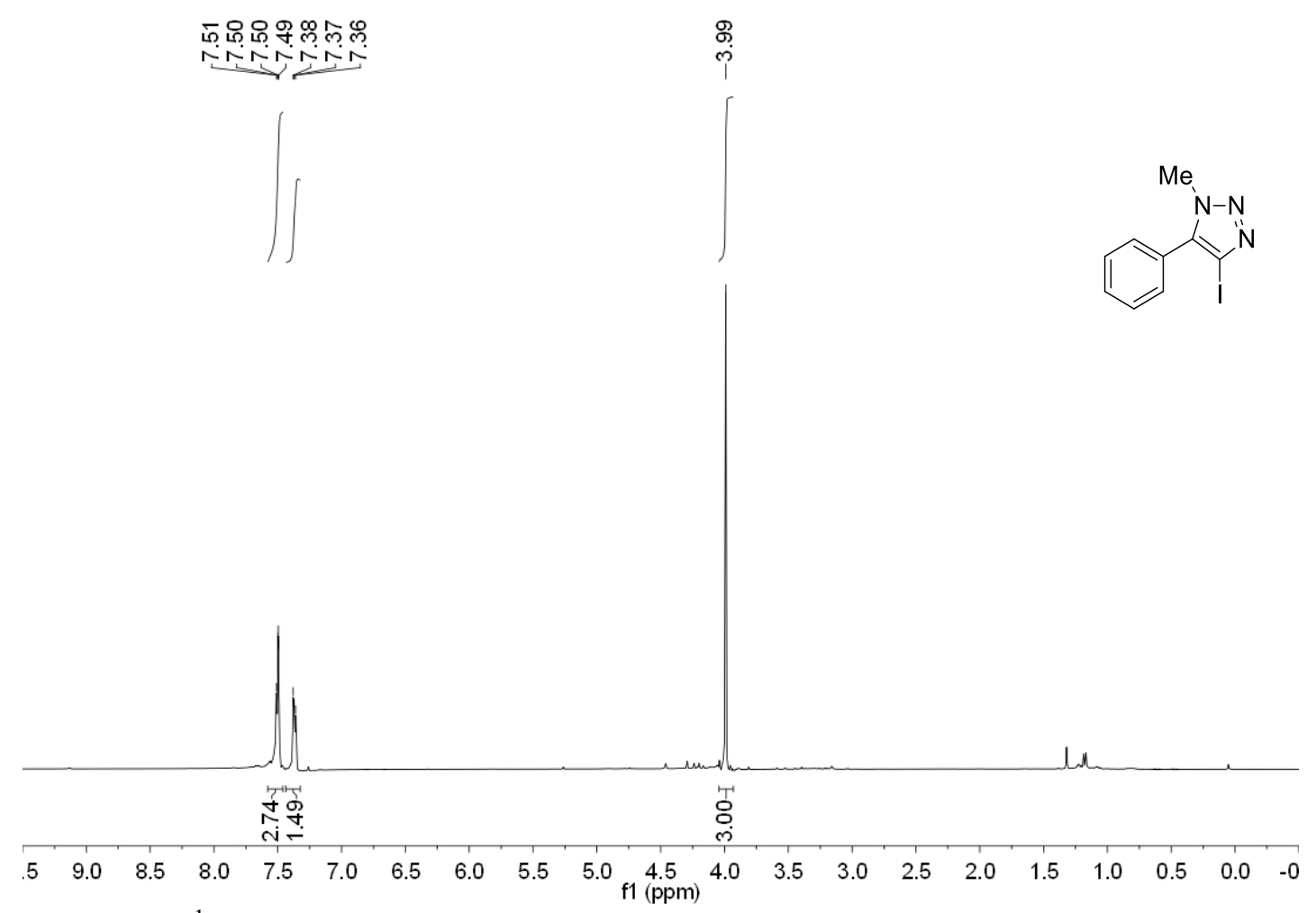

Spectrum 63. ${ }^{1} \mathrm{H}$ NMR $\left(400 \mathrm{MHz}, \mathrm{CDCl}_{3}\right)$ spectrum of compound $\mathbf{8 a}$.

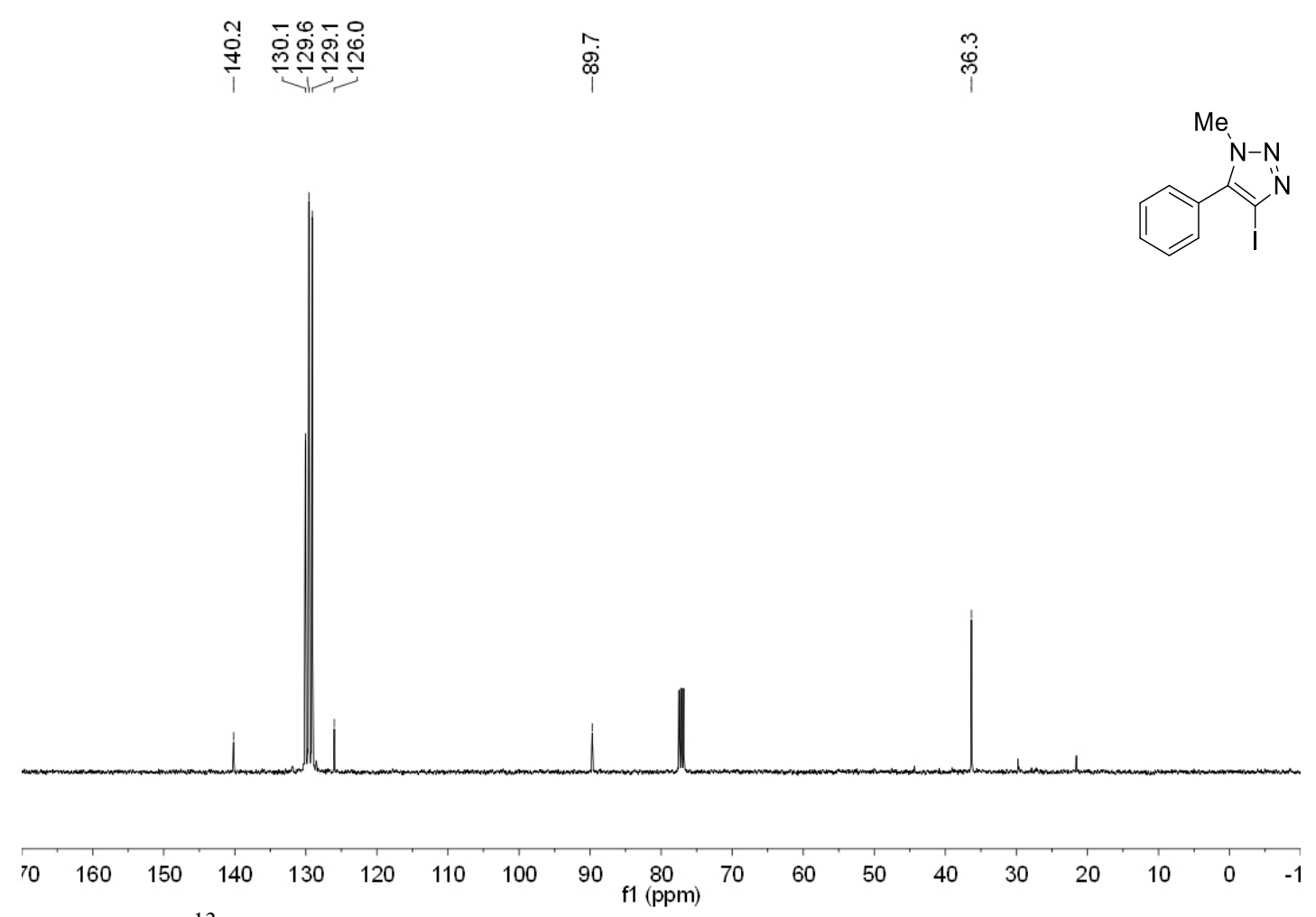

Spectrum 64. ${ }^{13} \mathrm{C}$ NMR (101 MHz, $\left.\mathrm{CDCl}_{3}\right)$ spectrum of compound 8a. 


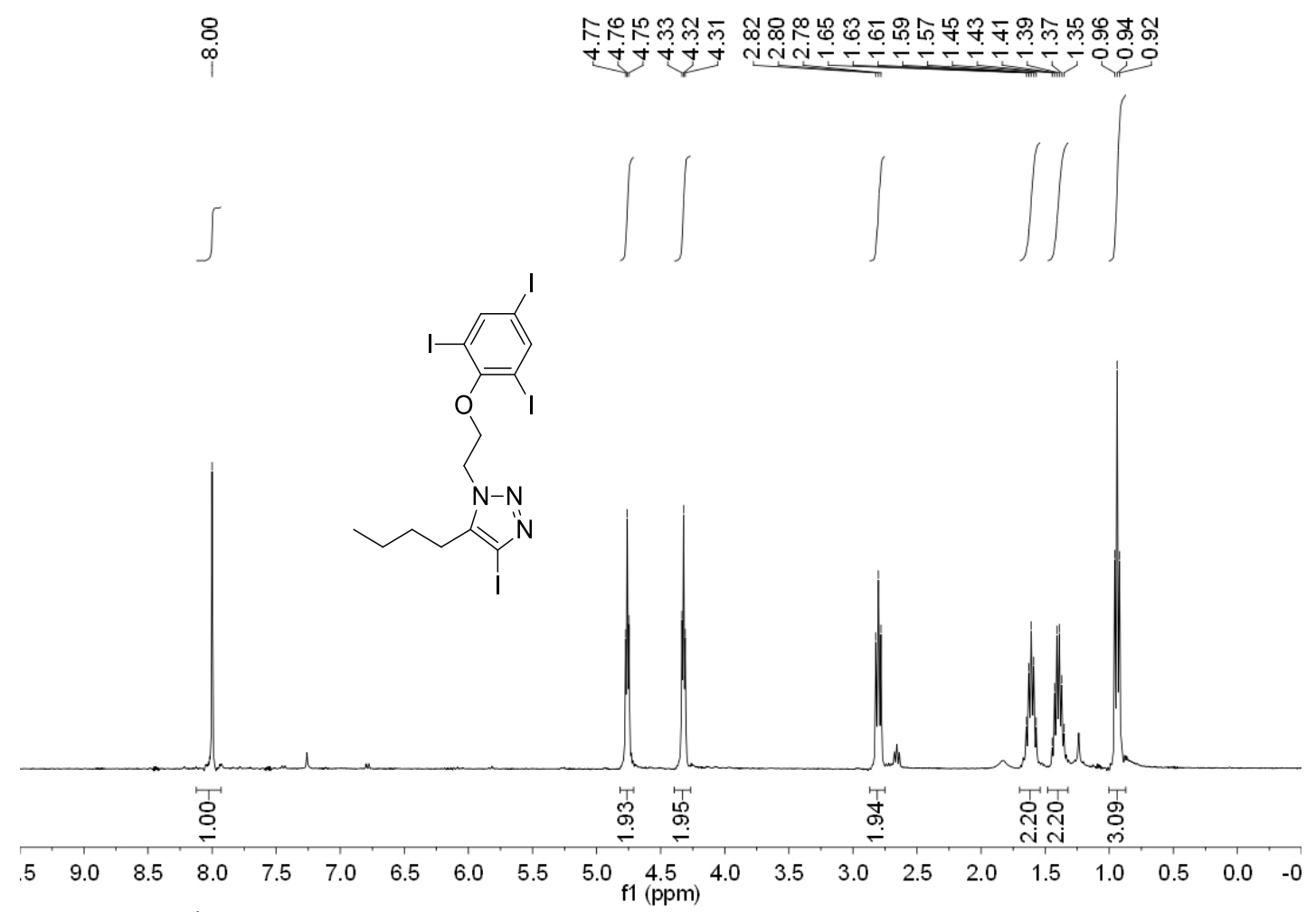

Spectrum 65. ${ }^{1} \mathrm{H}$ NMR $\left(400 \mathrm{MHz}, \mathrm{CDCl}_{3}\right)$ spectrum of compound $\mathbf{8 b}$.

皇
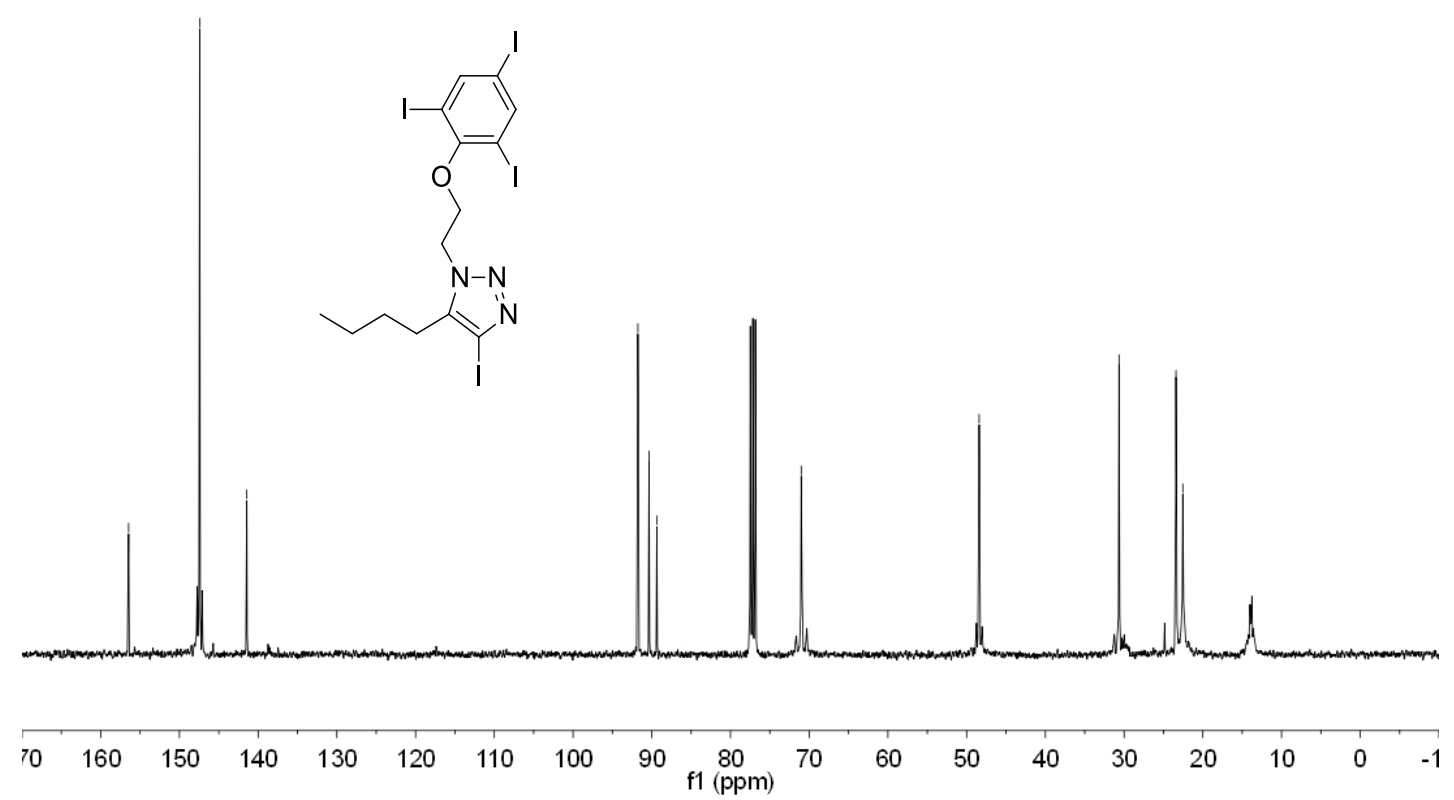

Spectrum 66. ${ }^{13} \mathrm{C}$ NMR (101 MHz, $\mathrm{CDCl}_{3}$ ) spectrum of compound $\mathbf{8 b}$. 


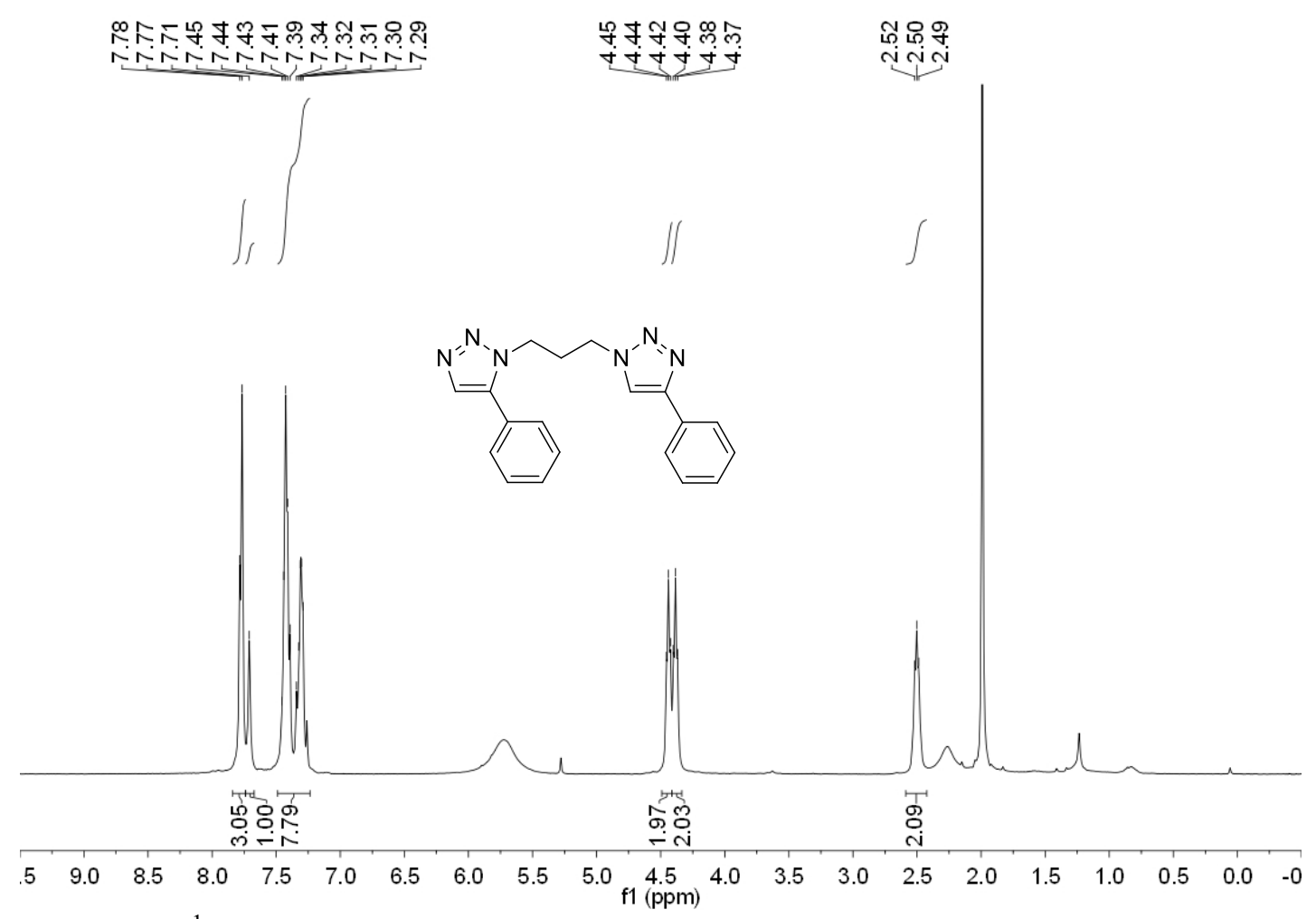

Spectrum 67. ${ }^{1} \mathrm{H}$ NMR (400 $\left.\mathrm{MHz}, \mathrm{CDCl}_{3}\right)$ spectrum of compound 9a.

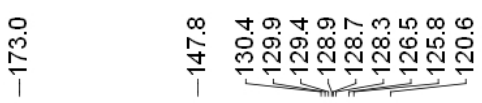

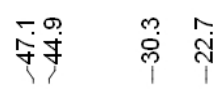
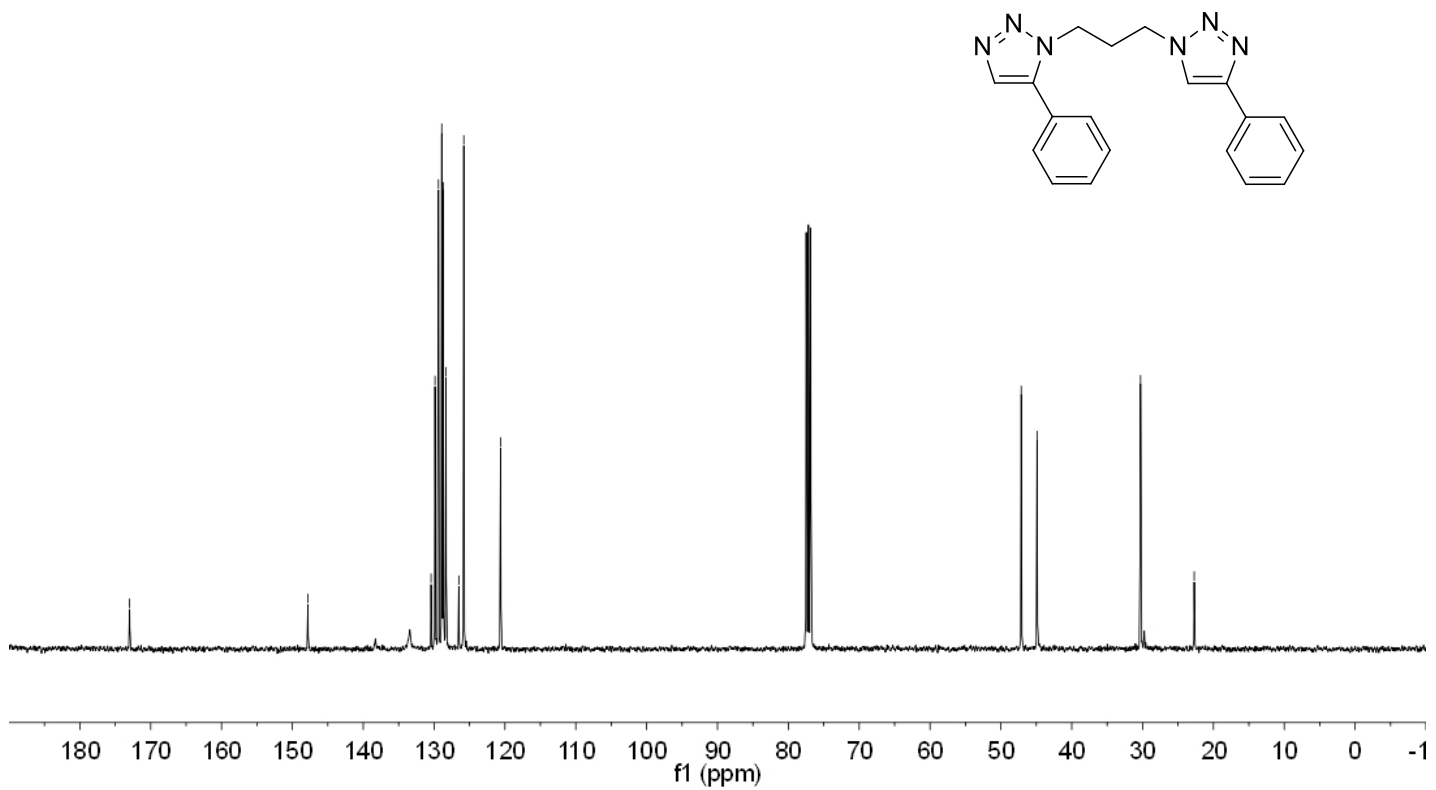

Spectrum 68. ${ }^{13} \mathrm{C}$ NMR (101 MHz, $\left.\mathrm{CDCl}_{3}\right)$ spectrum of compound 9a. 


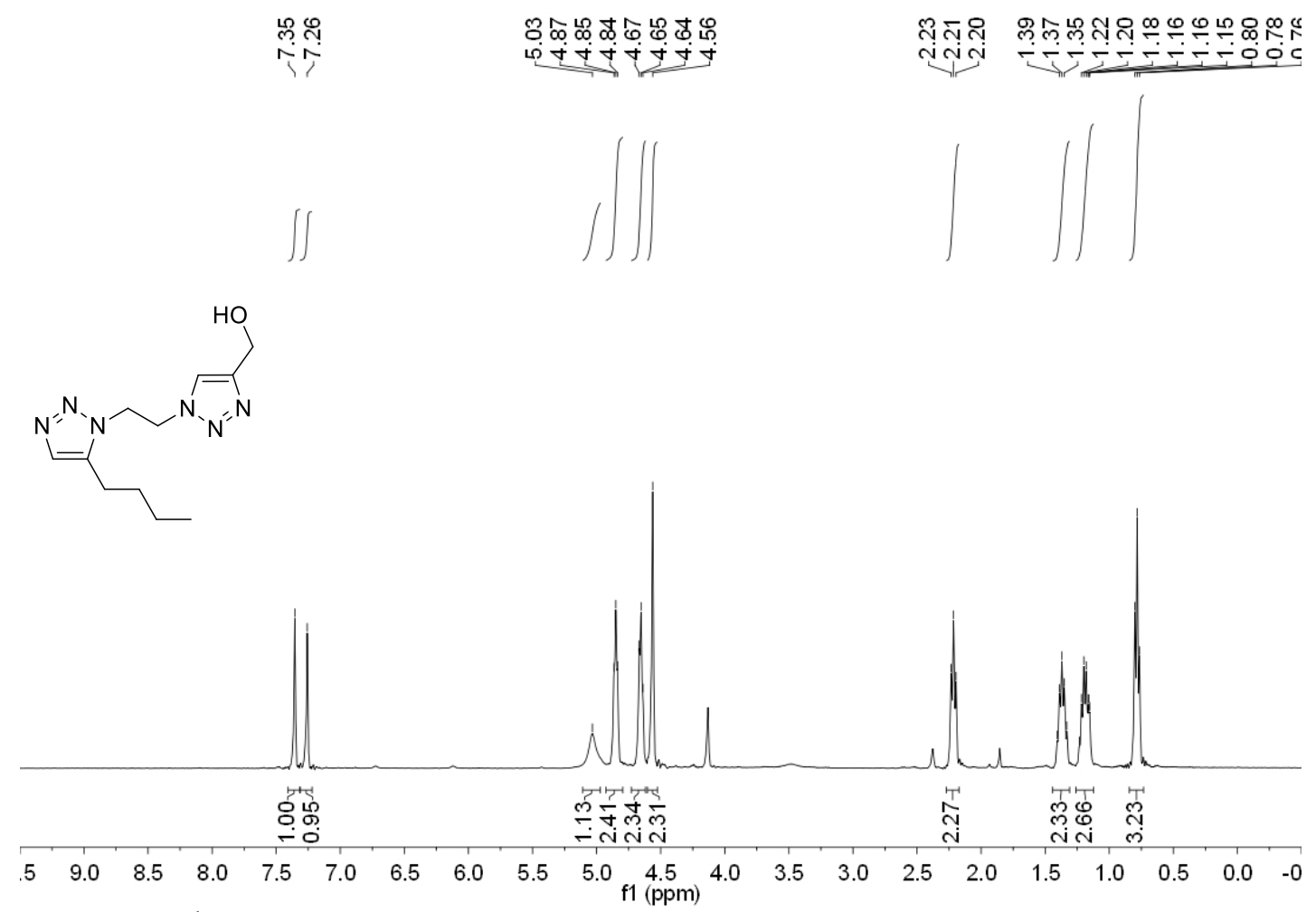

Spectrum 69. ${ }^{1} \mathrm{H}$ NMR $\left(400 \mathrm{MHz}, \mathrm{CDCl}_{3}\right)$ spectrum of compound $\mathbf{9 b}$.

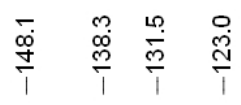

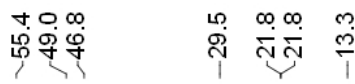
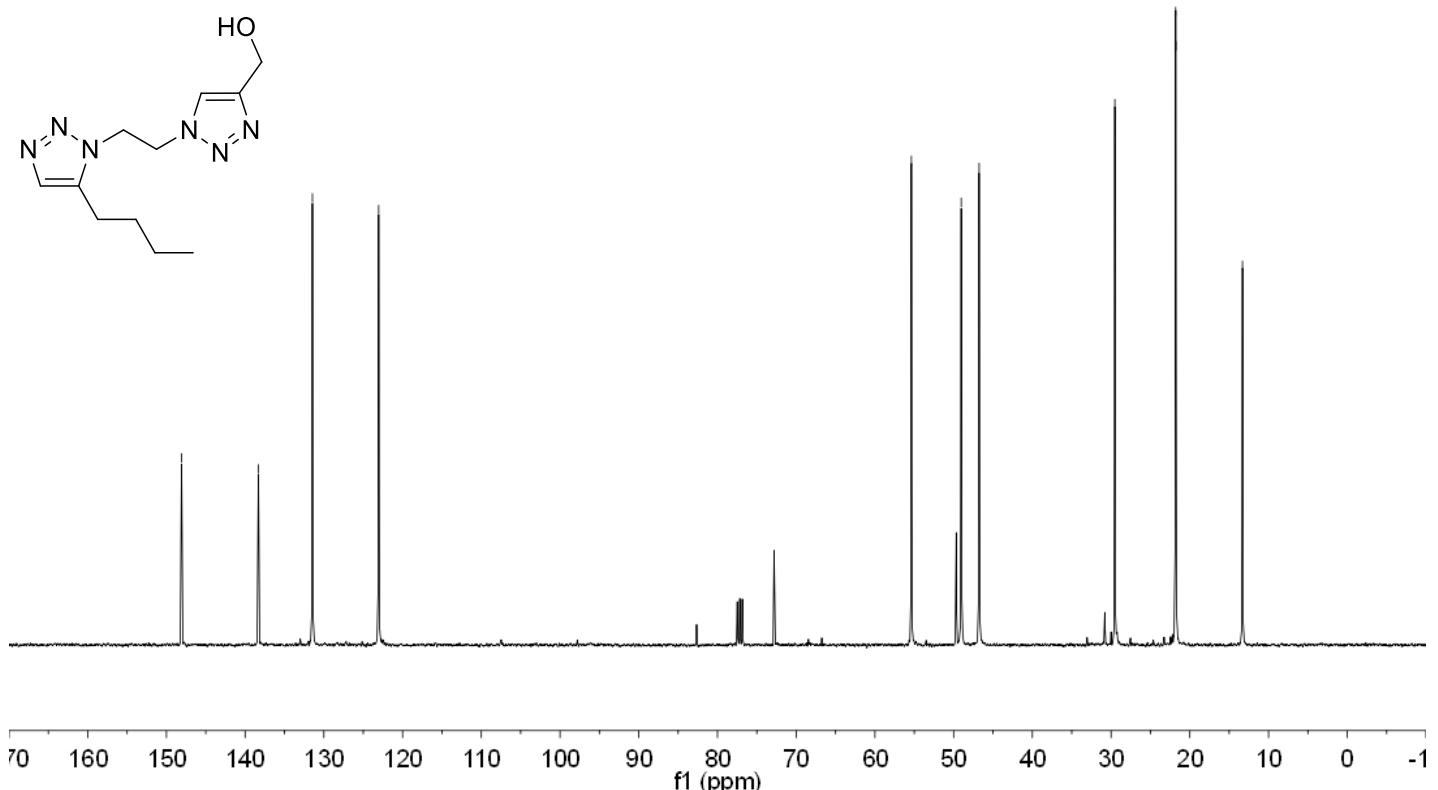

Spectrum 70. ${ }^{13} \mathrm{C}$ NMR (101 MHz, $\left.\mathrm{CDCl}_{3}\right)$ spectrum of compound $9 \mathbf{b}$. 


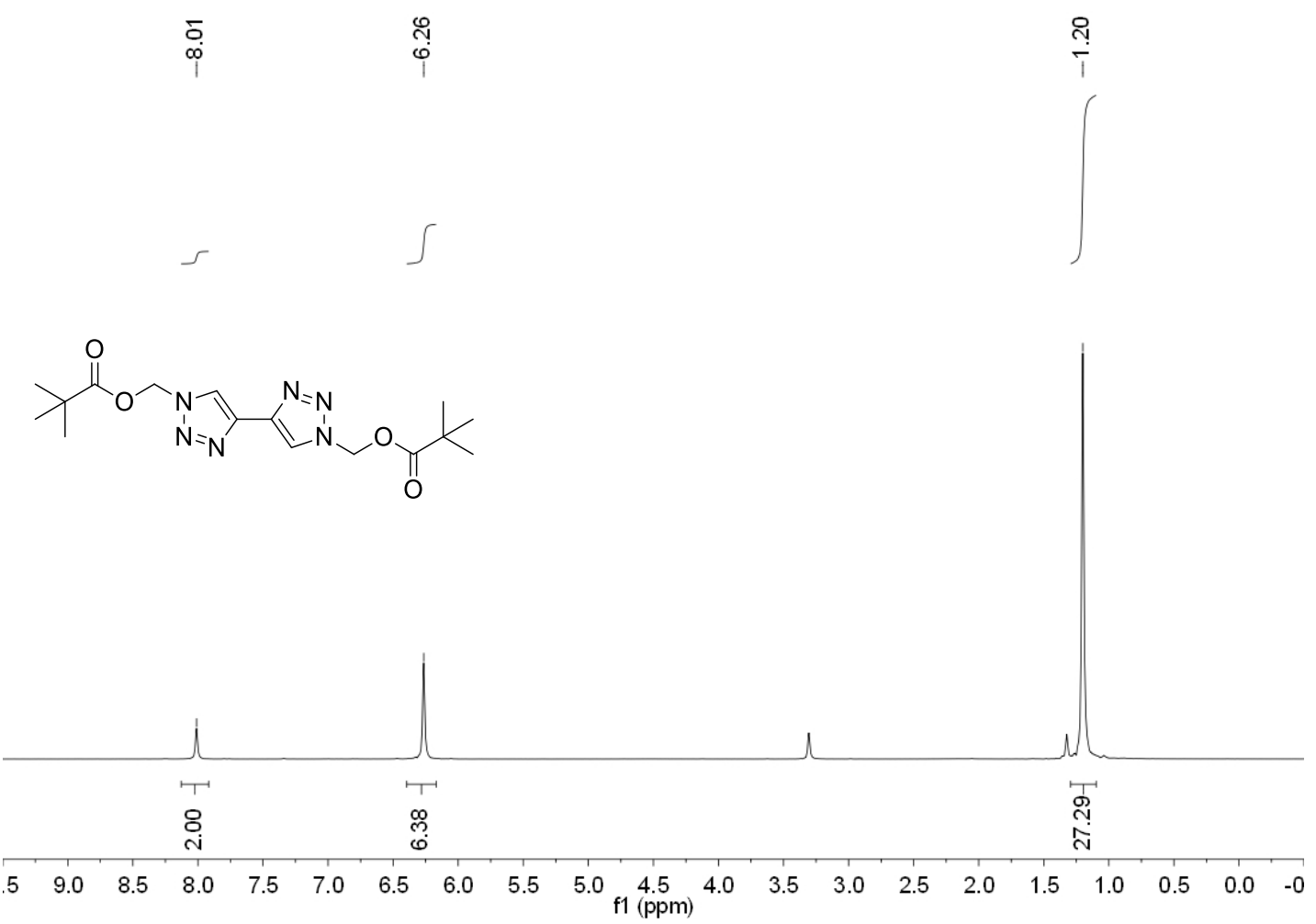

Spectrum 71. ${ }^{1} \mathrm{H}$ NMR $\left(400 \mathrm{MHz}, \mathrm{CDCl}_{3}\right)$ spectrum of compound 11.

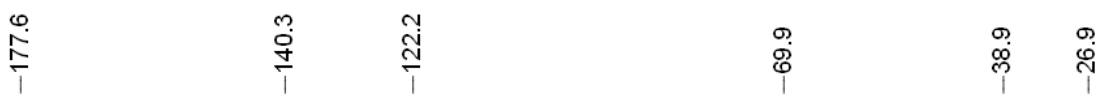
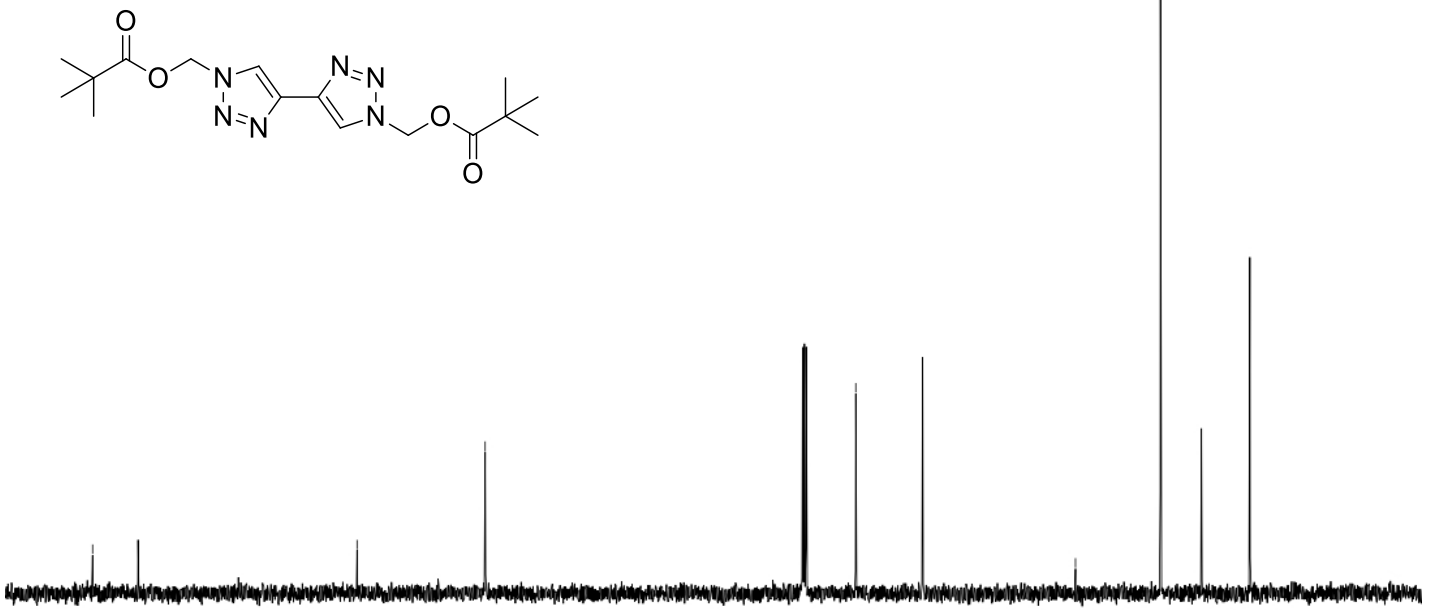

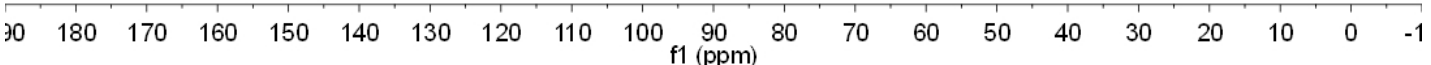

Spectrum 72. ${ }^{13} \mathrm{C} \mathrm{NMR}\left(126 \mathrm{MHz}, \mathrm{CDCl}_{3}\right)$ spectrum of compound 11. 


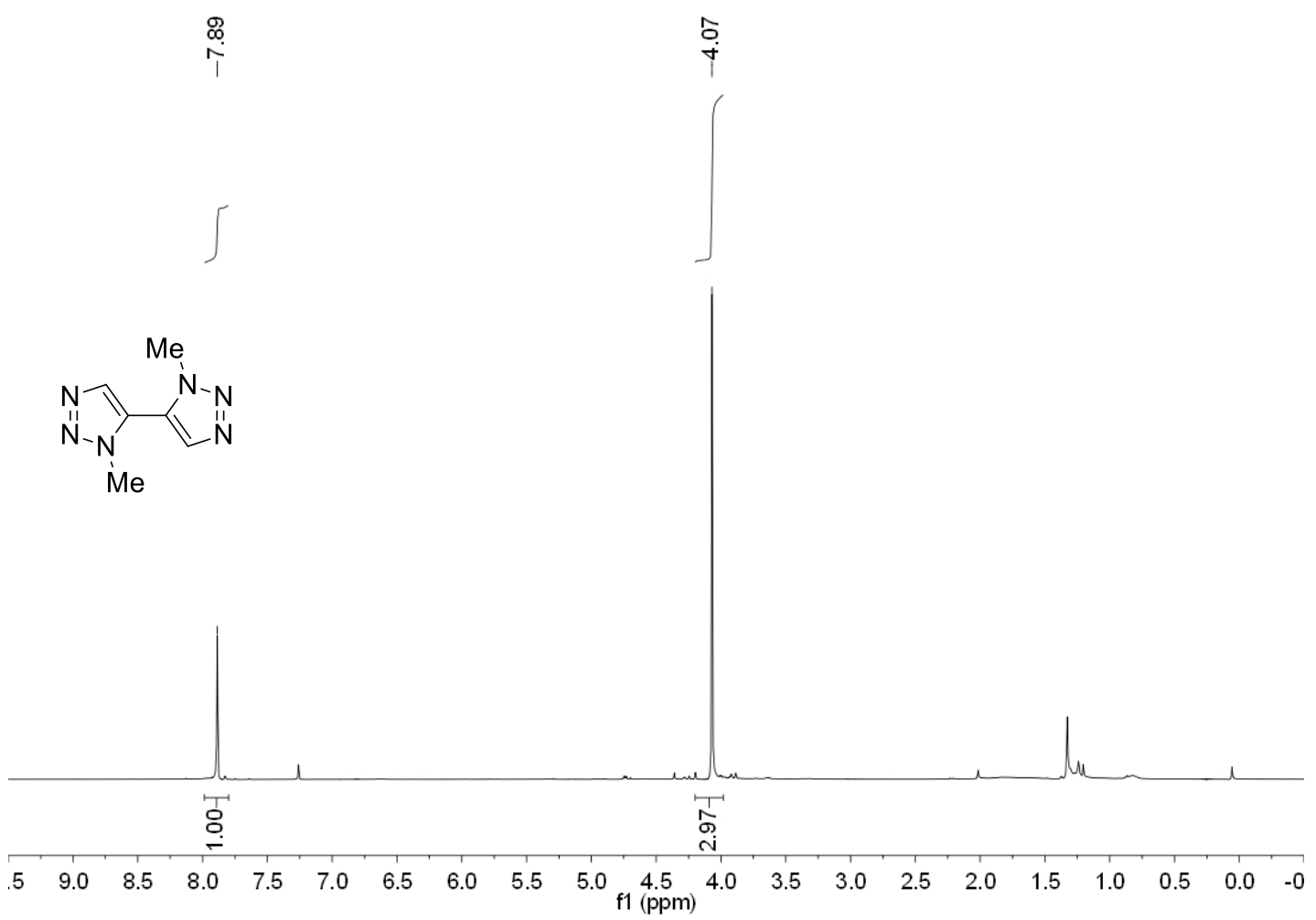

Spectrum 73. ${ }^{1} \mathrm{H}$ NMR (400 MHz, $\left.\mathrm{CDCl}_{3}\right)$ spectrum of compound 12.

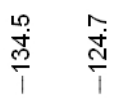

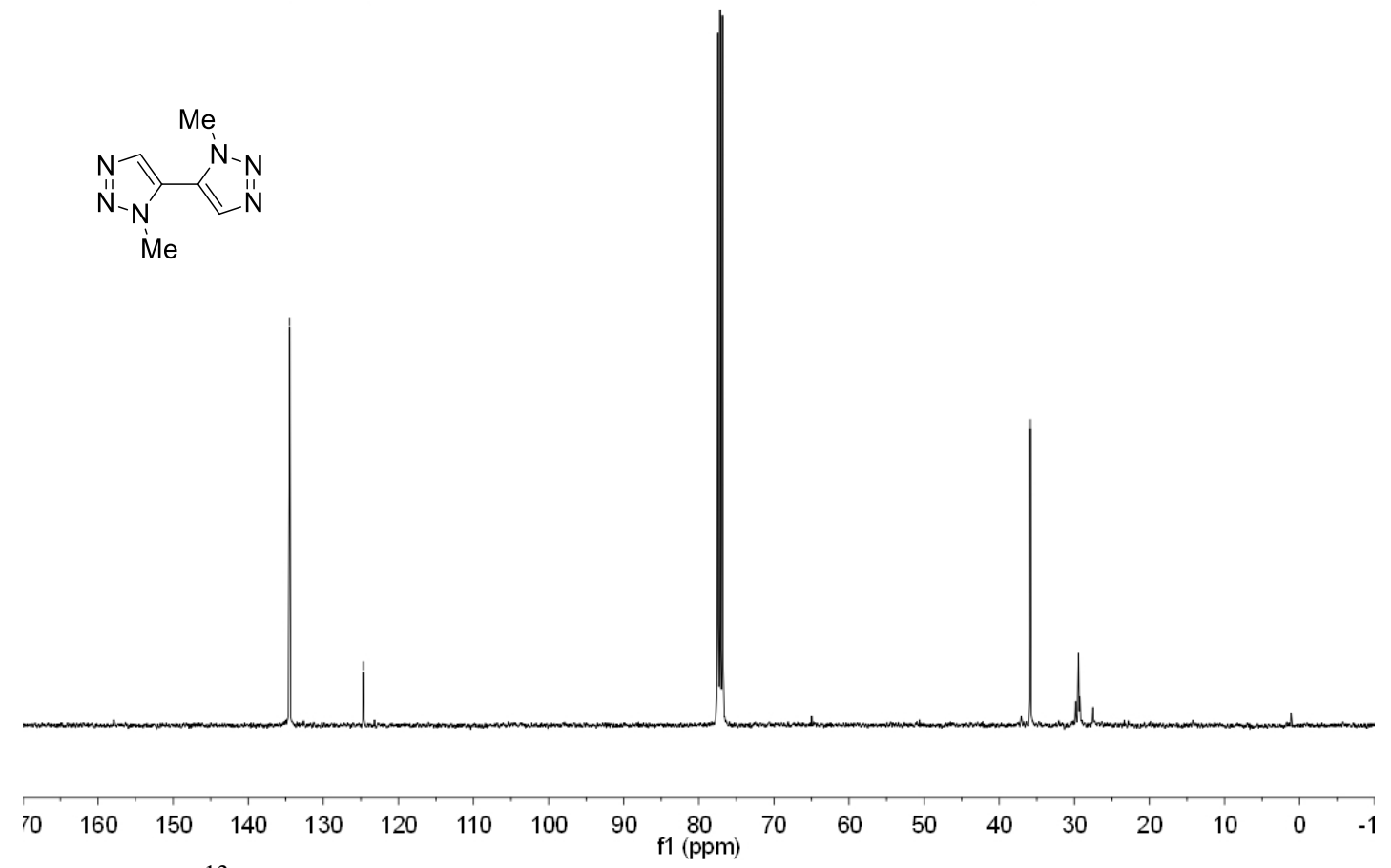

$\stackrel{\infty}{\stackrel{m}{\varphi}}$

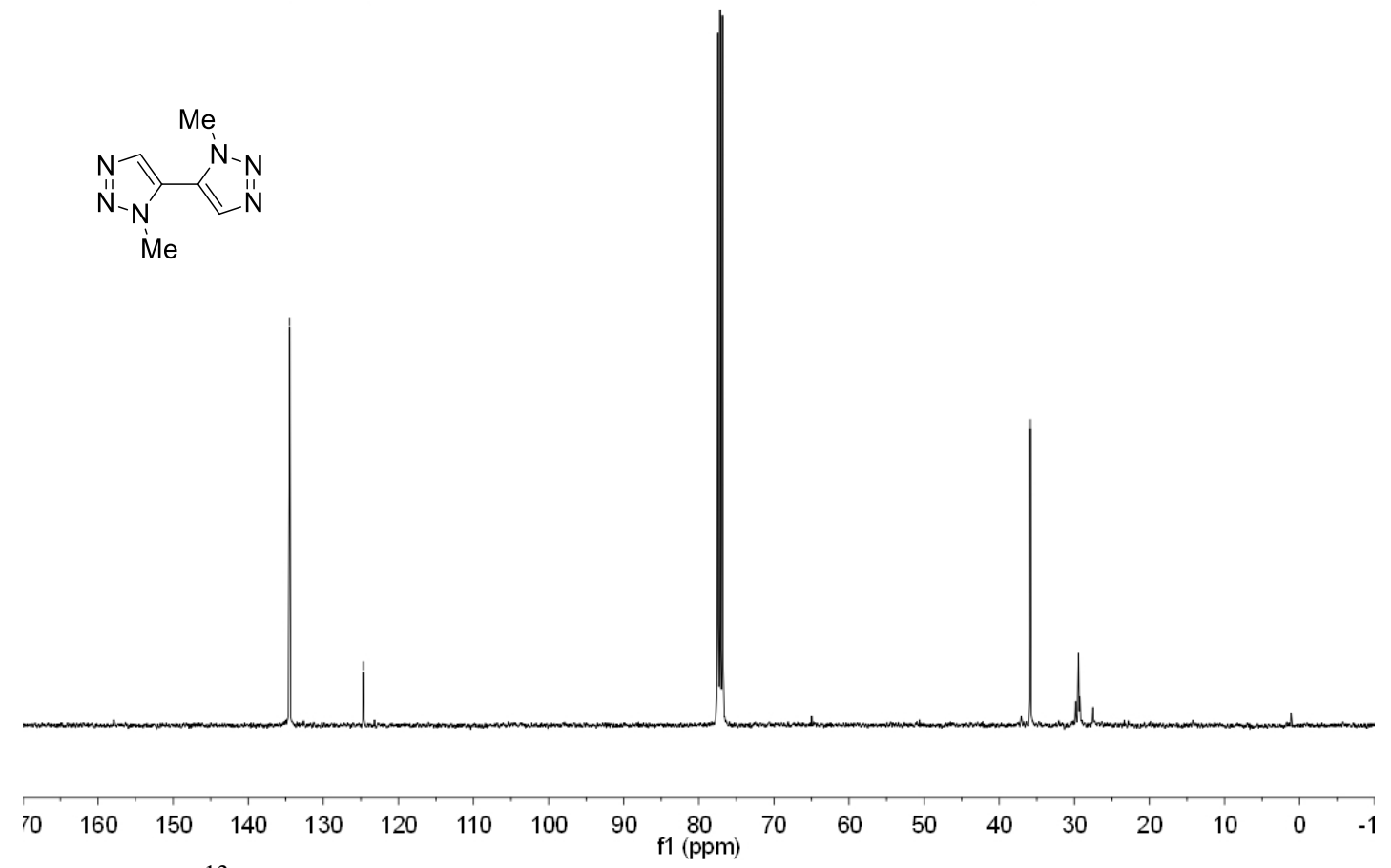

๓ं

Spectrum 74. ${ }^{13} \mathrm{C}$ NMR (101 MHz, $\left.\mathrm{CDCl}_{3}\right)$ spectrum of compound 12. 


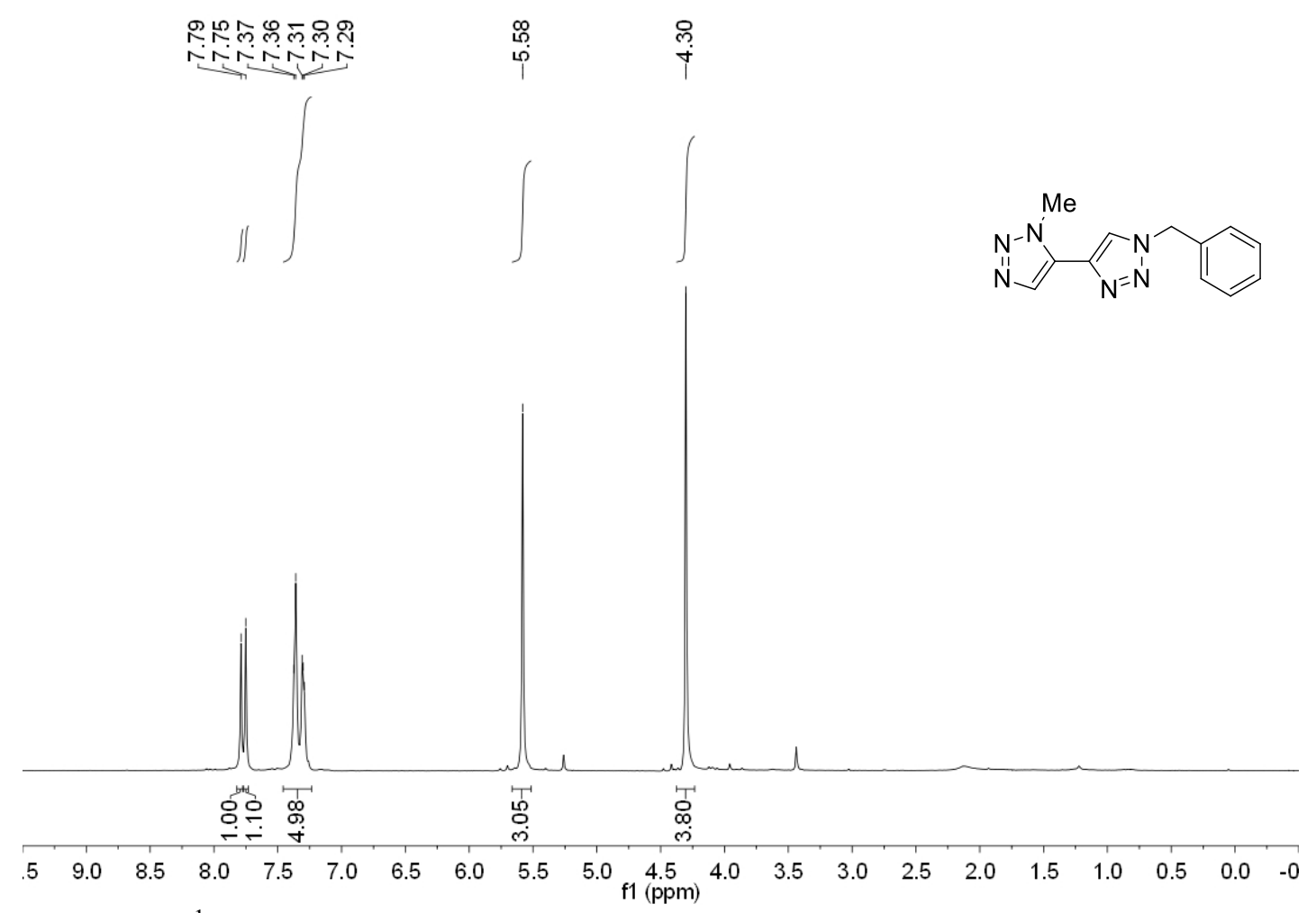

Spectrum 75. ${ }^{1} \mathrm{H} \mathrm{NMR}\left(400 \mathrm{MHz}, \mathrm{CDCl}_{3}\right)$ spectrum of compound $\mathbf{1 3 a}$.
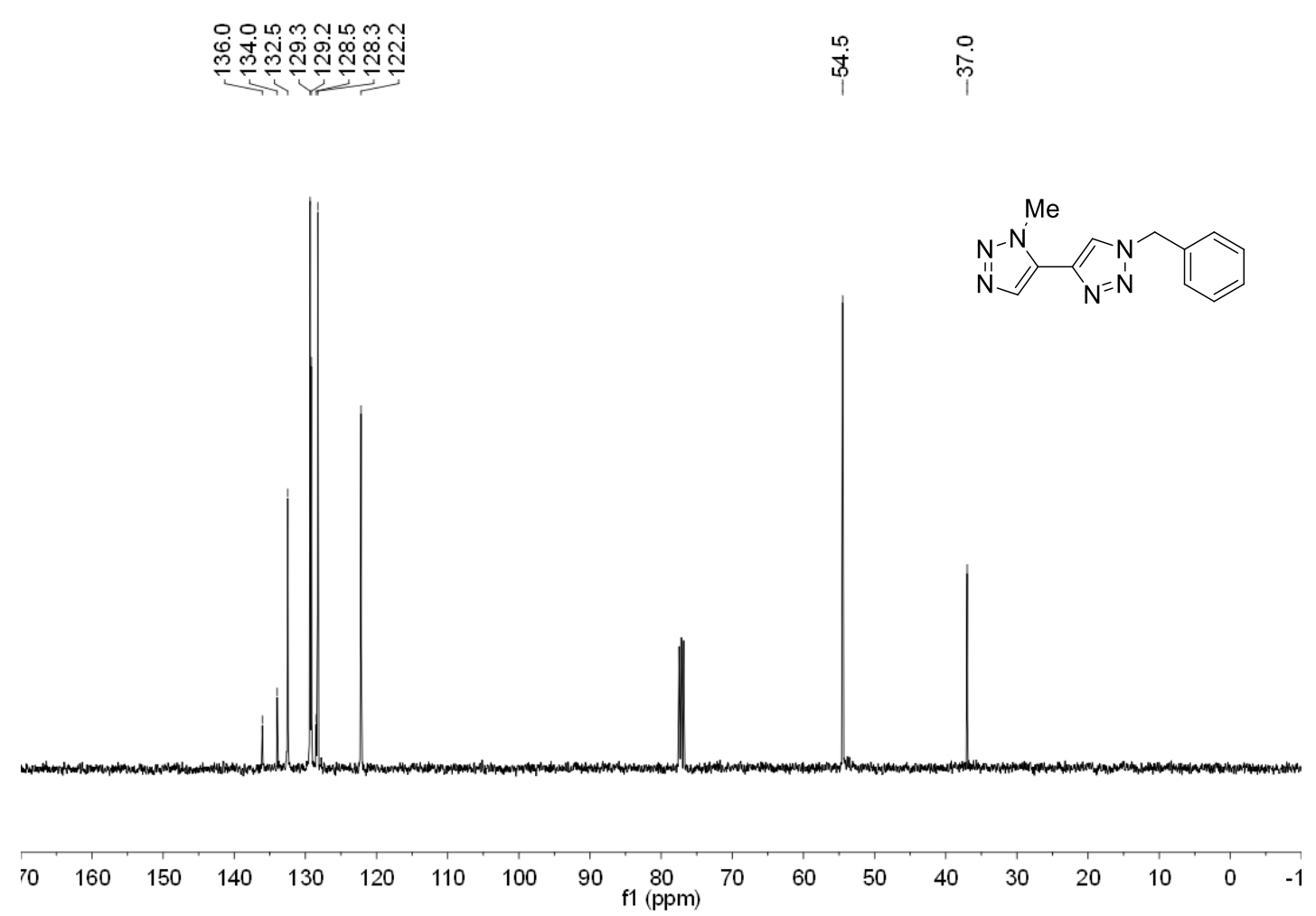

Spectrum 76. ${ }^{13} \mathrm{C}$ NMR (101 MHz, $\mathrm{CDCl}_{3}$ ) spectrum of compound $\mathbf{1 3 a}$. 


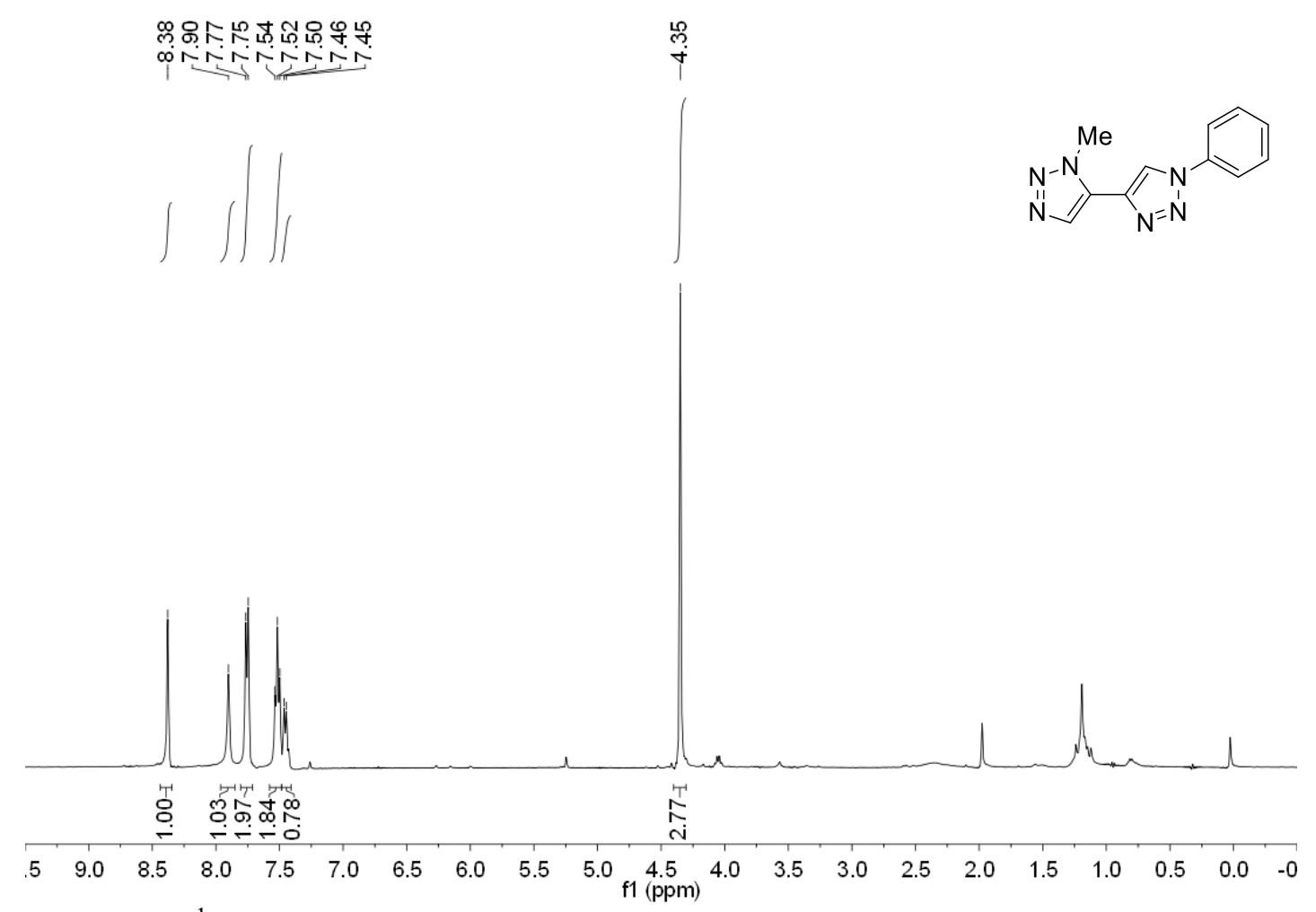

Spectrum 77. ${ }^{1} \mathrm{H} \mathrm{NMR}\left(400 \mathrm{MHz}, \mathrm{CDCl}_{3}\right)$ spectrum of compound $\mathbf{1 3 b}$.
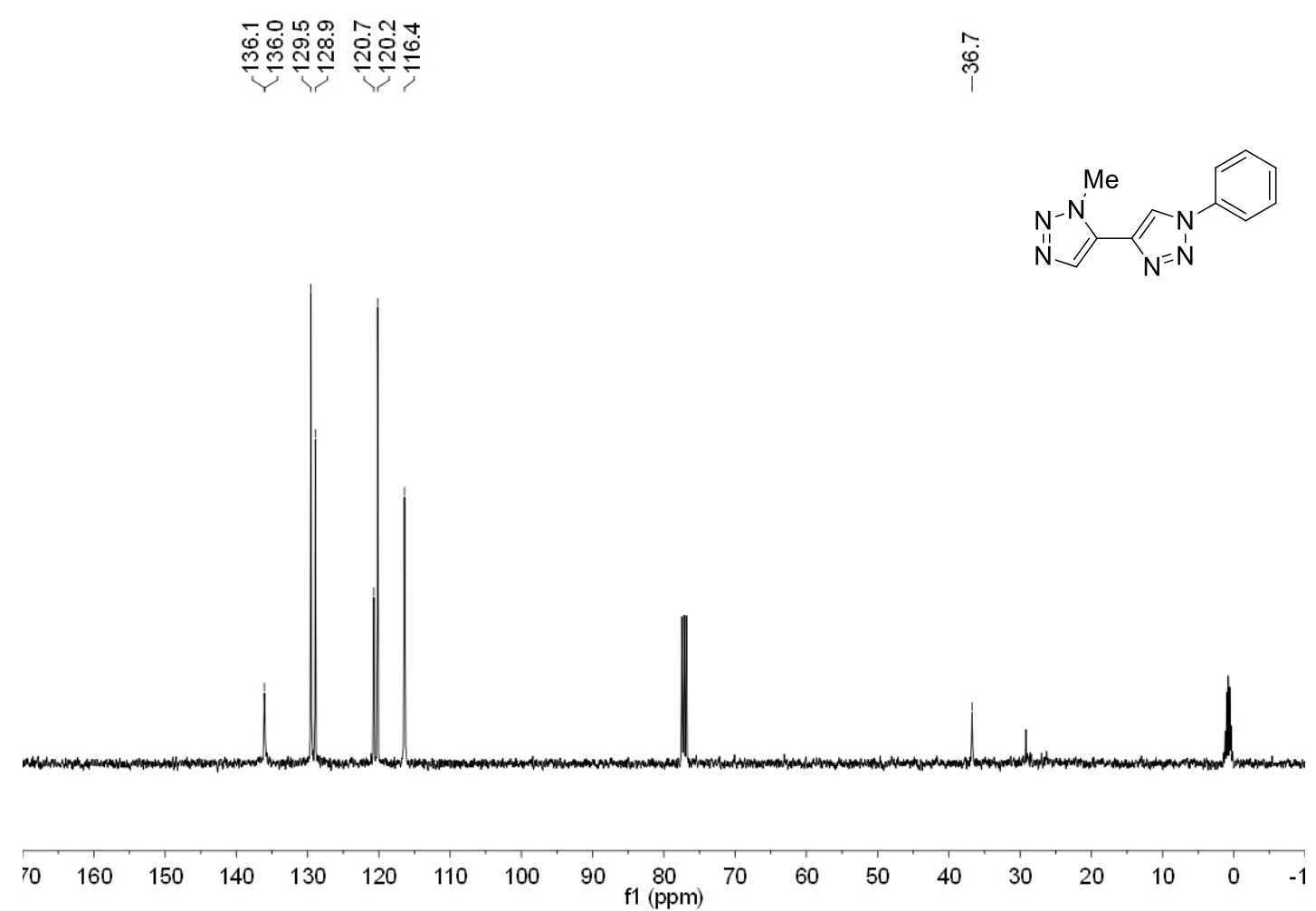

Spectrum 78. ${ }^{13} \mathrm{C}$ NMR $\left(101 \mathrm{MHz}, \mathrm{CDCl}_{3}\right)$ spectrum of compound $\mathbf{1 3 b}$. 


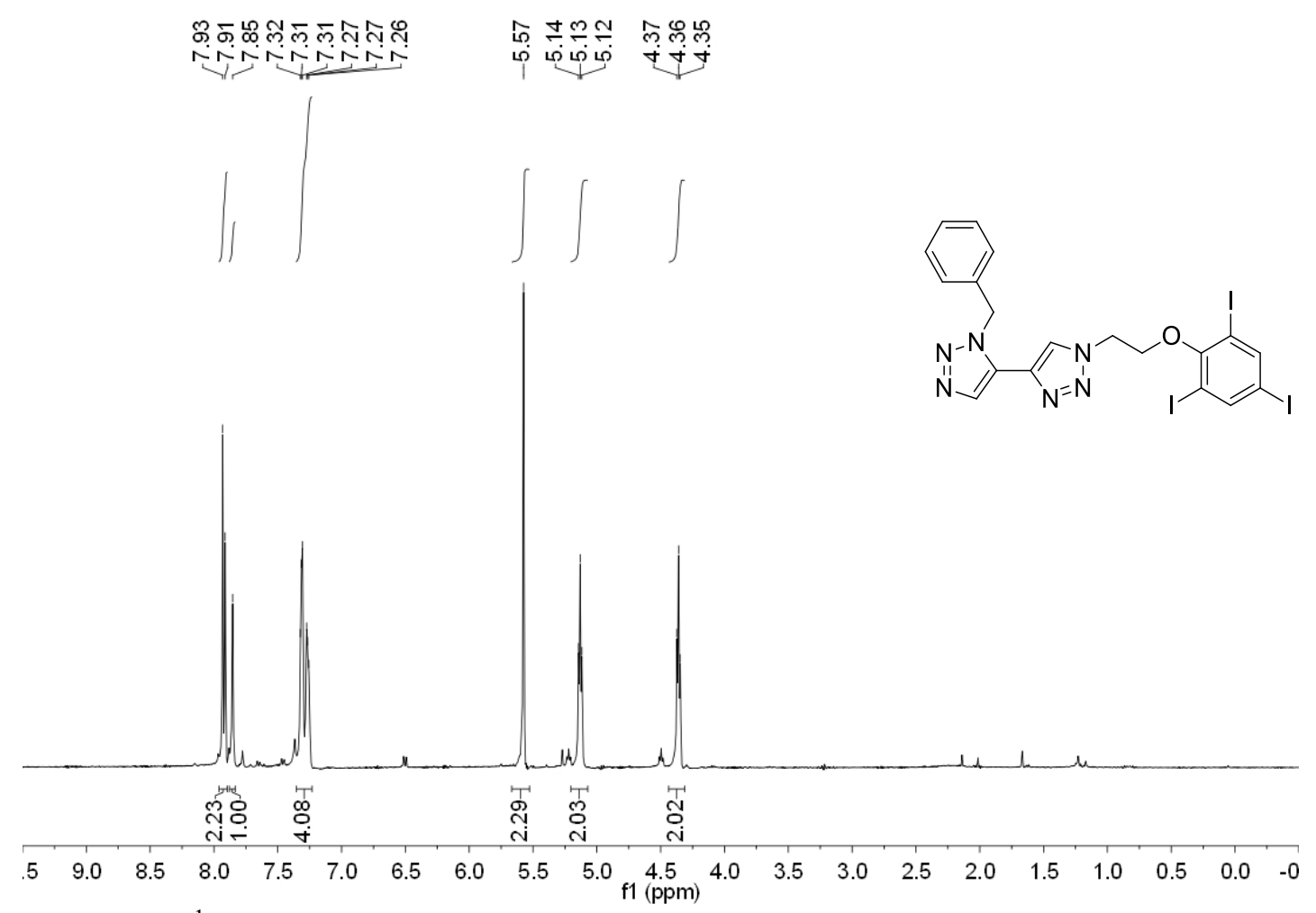

Spectrum 79. ${ }^{1} \mathrm{H} \mathrm{NMR}\left(400 \mathrm{MHz}, \mathrm{CDCl}_{3}\right)$ spectrum of compound $\mathbf{1 3 c}$.
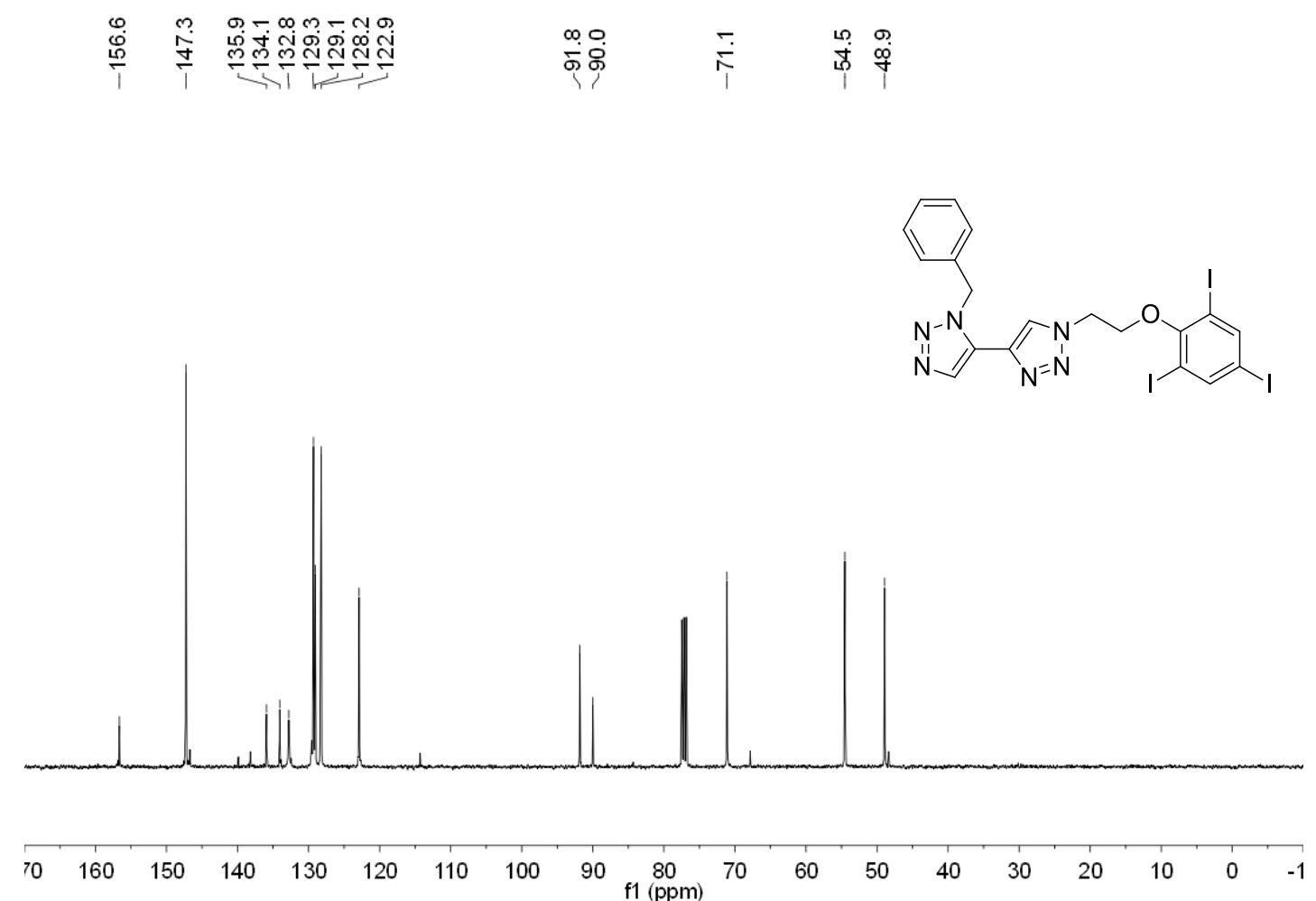

Spectrum 80. ${ }^{13} \mathrm{C}$ NMR (101 MHz, $\left.\mathrm{CDCl}_{3}\right)$ spectrum of compound $\mathbf{1 3 c}$. 


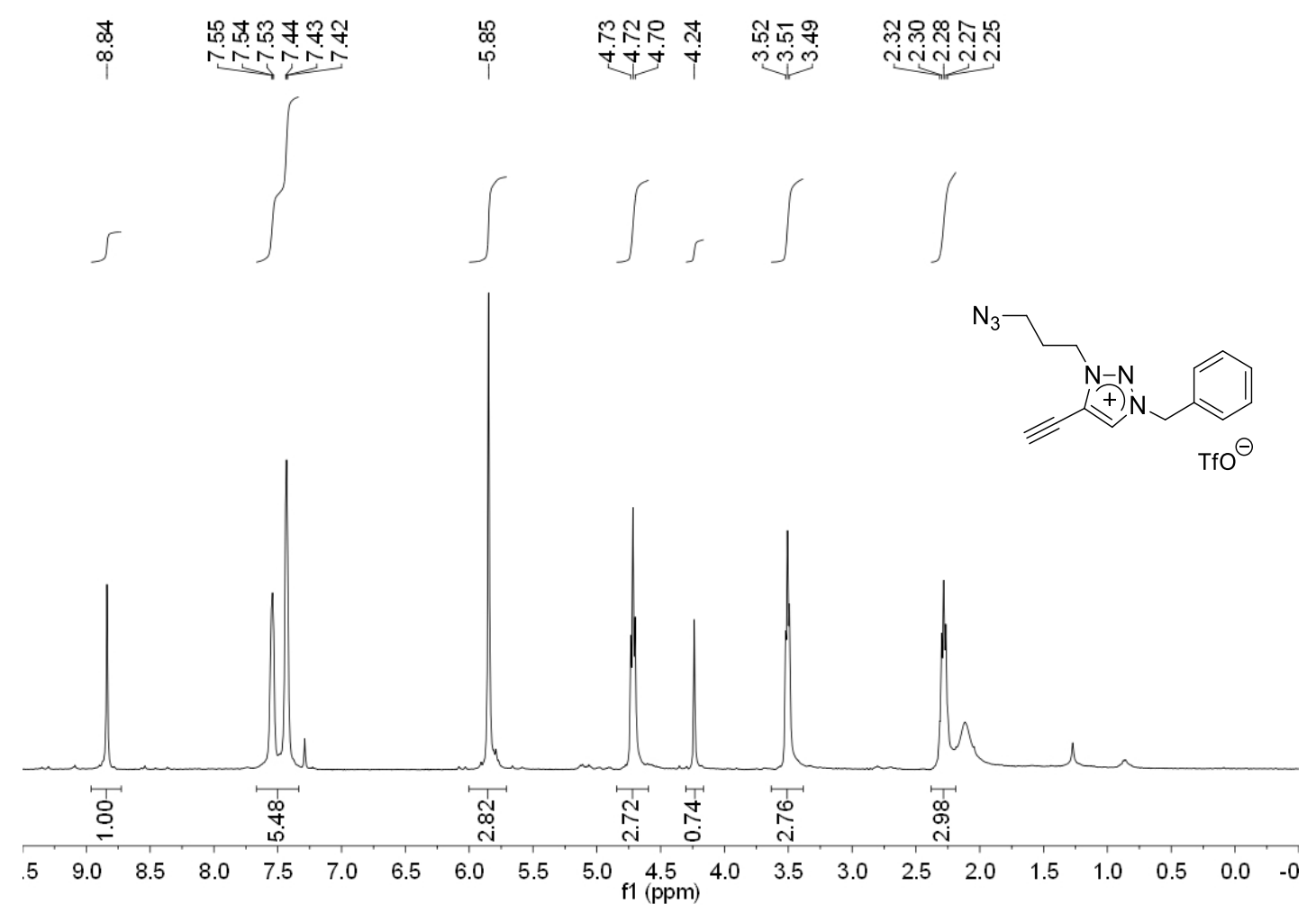

Spectrum 81. ${ }^{1} \mathrm{H}$ NMR $\left(400 \mathrm{MHz}, \mathrm{CDCl}_{3}\right)$ spectrum of compound 15.
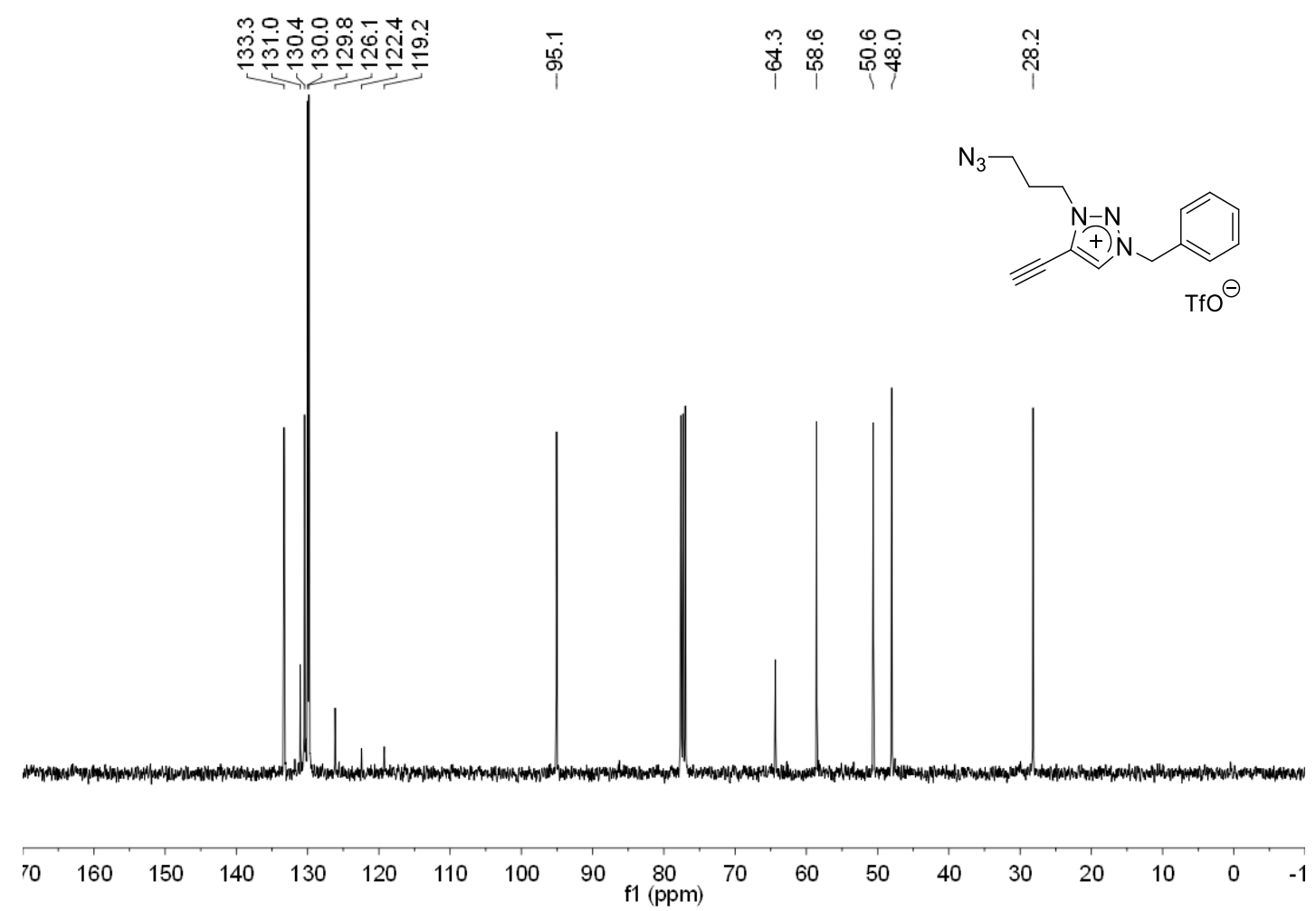

Spectrum 82. ${ }^{13} \mathrm{C}$ NMR $\left(101 \mathrm{MHz}, \mathrm{CDCl}_{3}\right)$ spectrum of compound 15. 


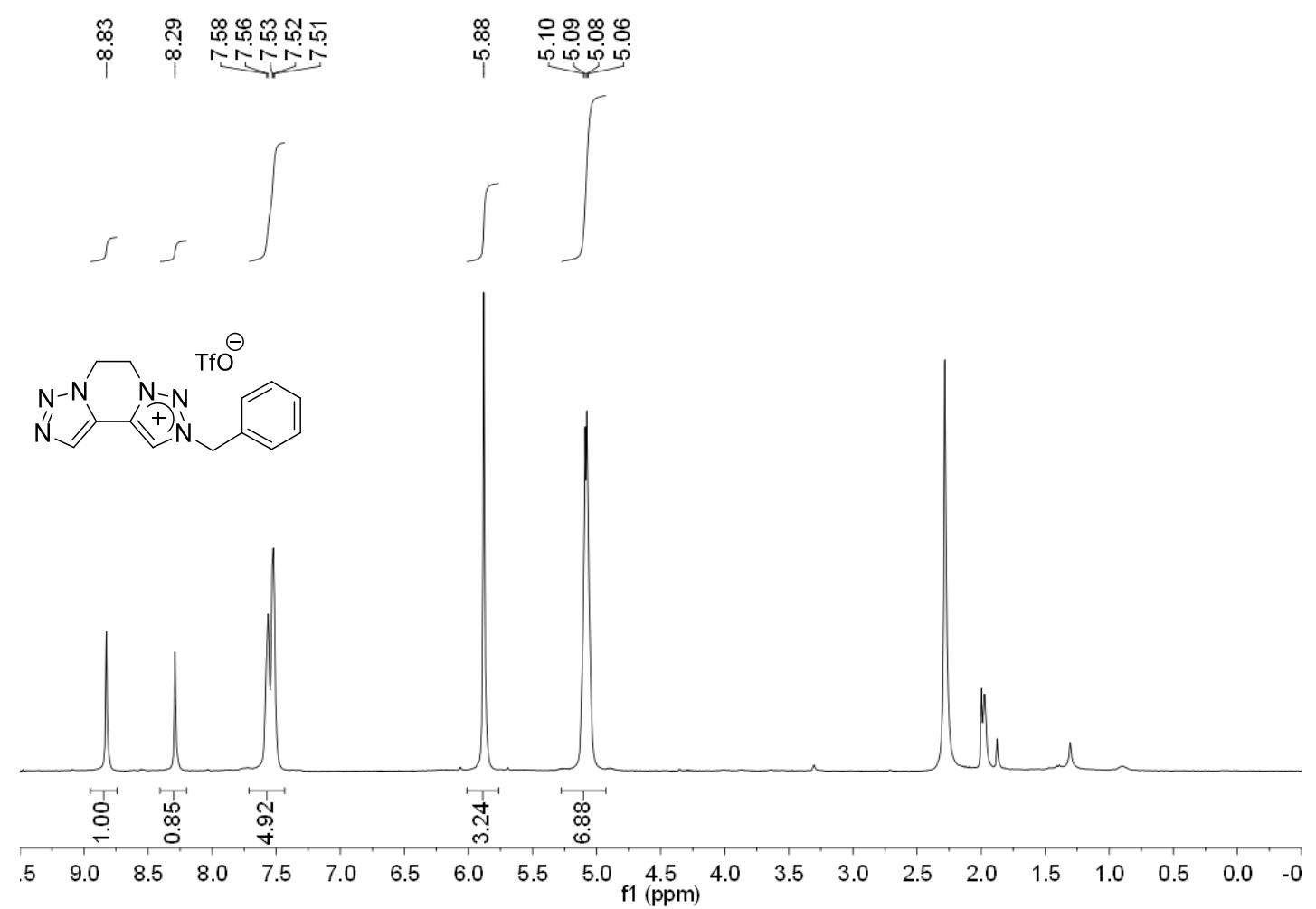

Spectrum 83. ${ }^{1} \mathrm{H}$ NMR (400 MHz, MeCN-d ${ }_{3}$ ) spectrum of compound 17.

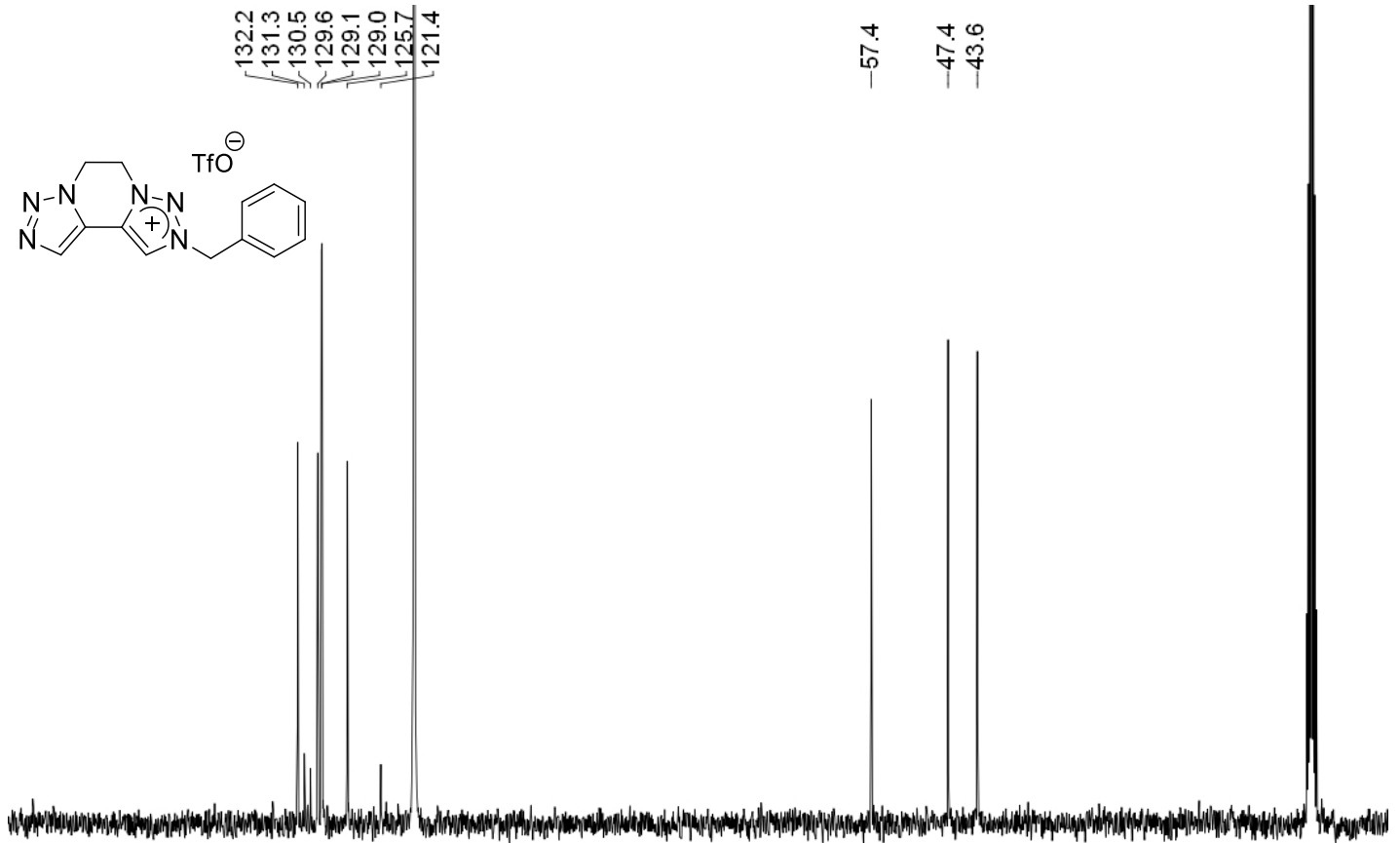

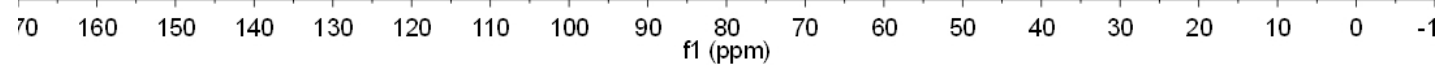

Spectrum 84. ${ }^{13} \mathrm{C}$ NMR $\left(101 \mathrm{MHz}, \mathrm{MeCN}-\mathrm{d}_{3}\right)$ spectrum of compound 17. 


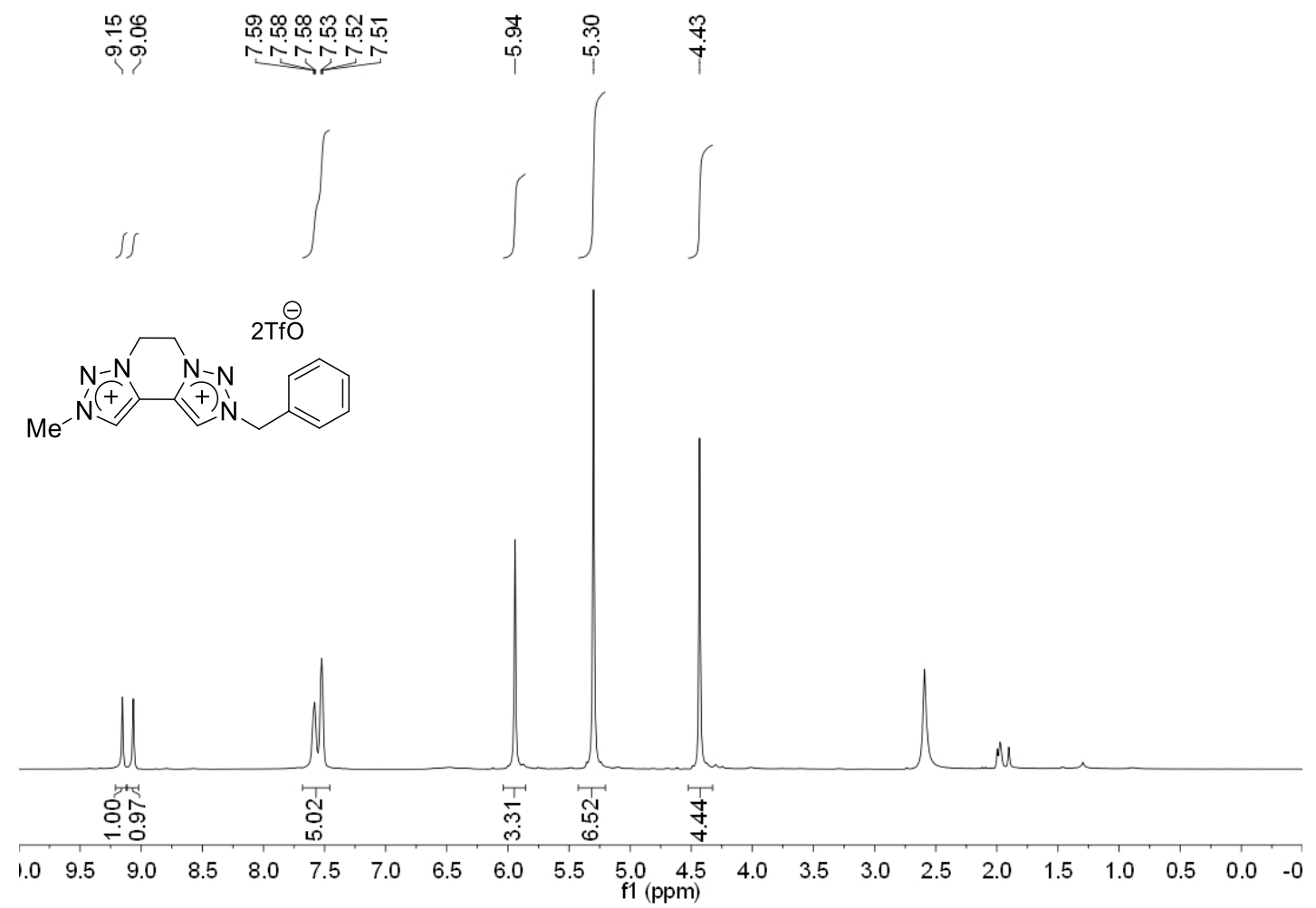

Spectrum 85. ${ }^{1} \mathrm{H}$ NMR (400 MHz, MeCN-d ${ }_{3}$ ) spectrum of compound 18.

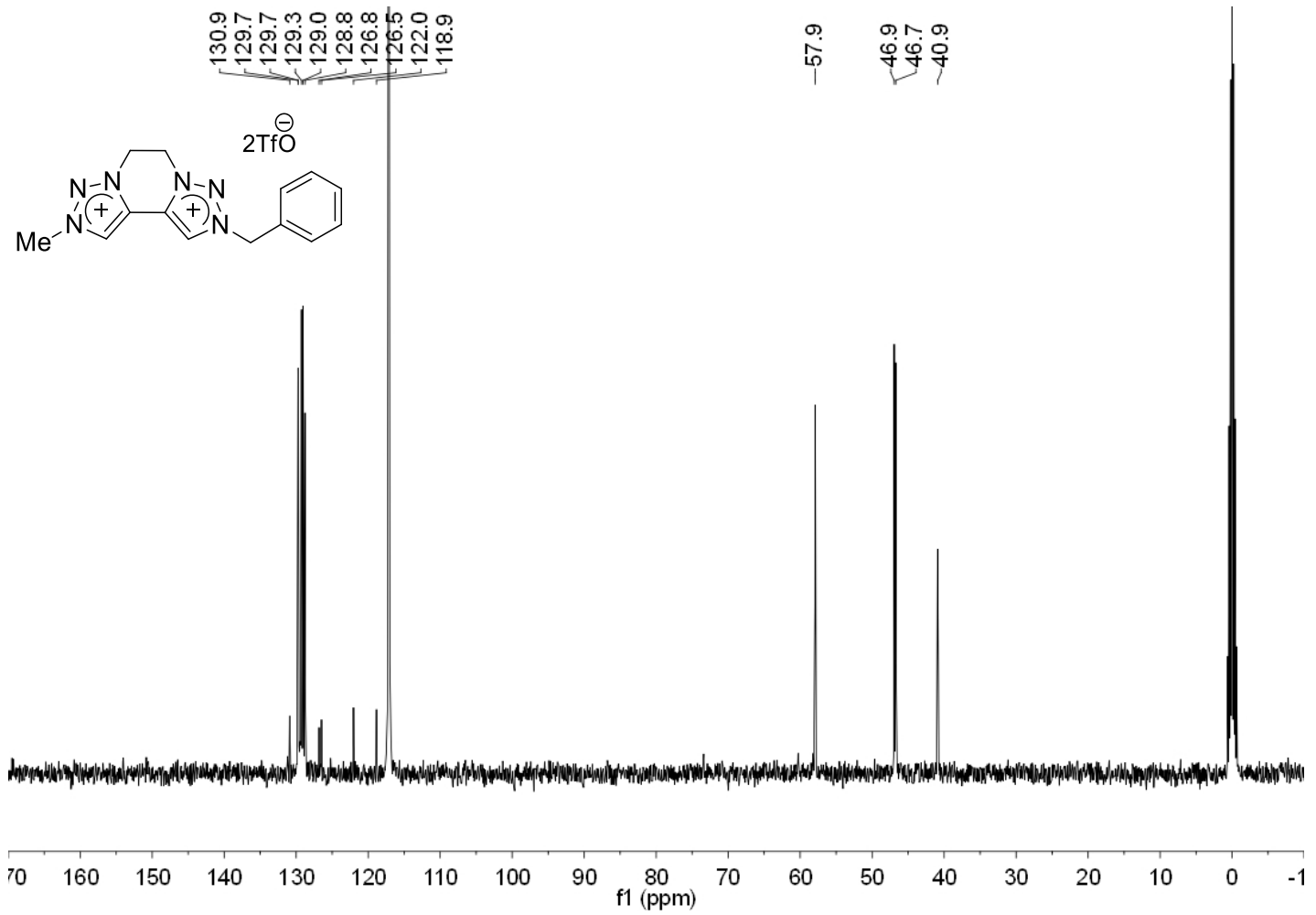

Spectrum 86. ${ }^{13} \mathrm{C}$ NMR (101 MHz, MeCN-d 3 ) spectrum of compound 18. 
3. N-Dealkylation of 1-benzyl-3-methyl-4-phenyl-1,2,3-triazolium salt 2n.

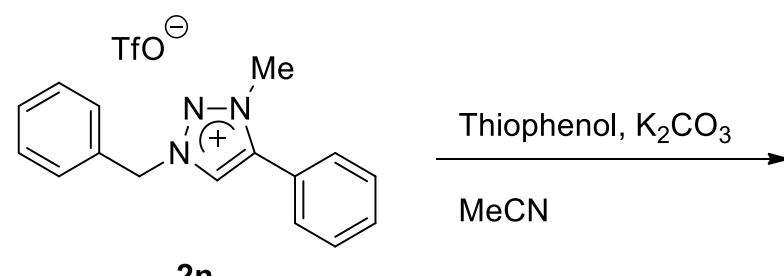

2n

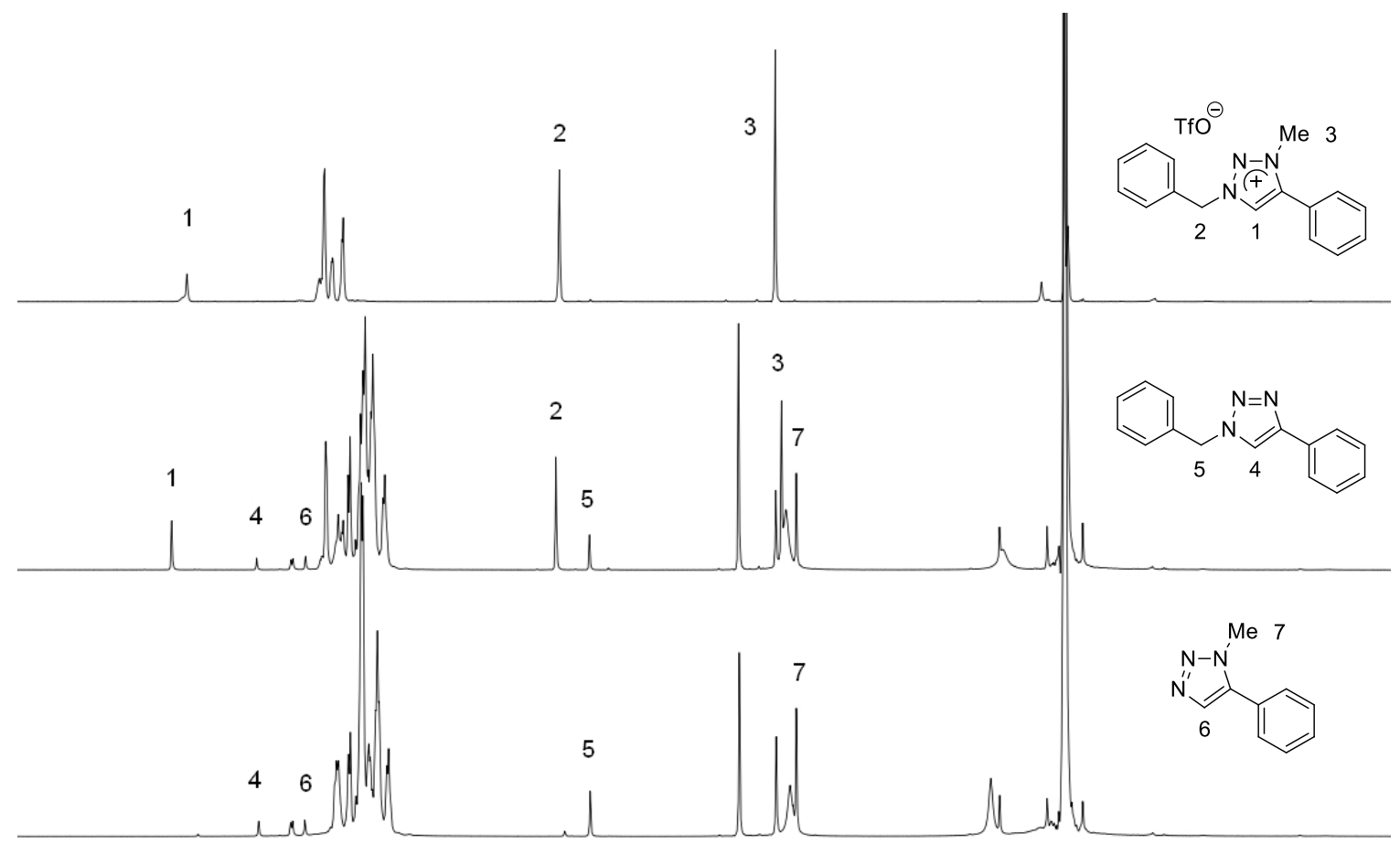

$0 \quad 9.5 \quad 9.0$

Figure S1. ${ }^{1} \mathrm{H}$ NMR $\left(500 \mathrm{MHz}, \mathrm{MeCN}-\mathrm{d}_{3}\right)$ spectra of the non selective N-demethylation reaction of 1-benzyl-3-methyl-4-phenyl-1,2,3-triazolium trifluoromethanesilfonate $\mathbf{2 n}$ in the presence of thiophenol and $\mathrm{K}_{2} \mathrm{CO}_{3}$. 


\section{N-Alkylation/dealkylation of 1-pivaloyloxymethyl-1,2,3-triazole $1 i$.}
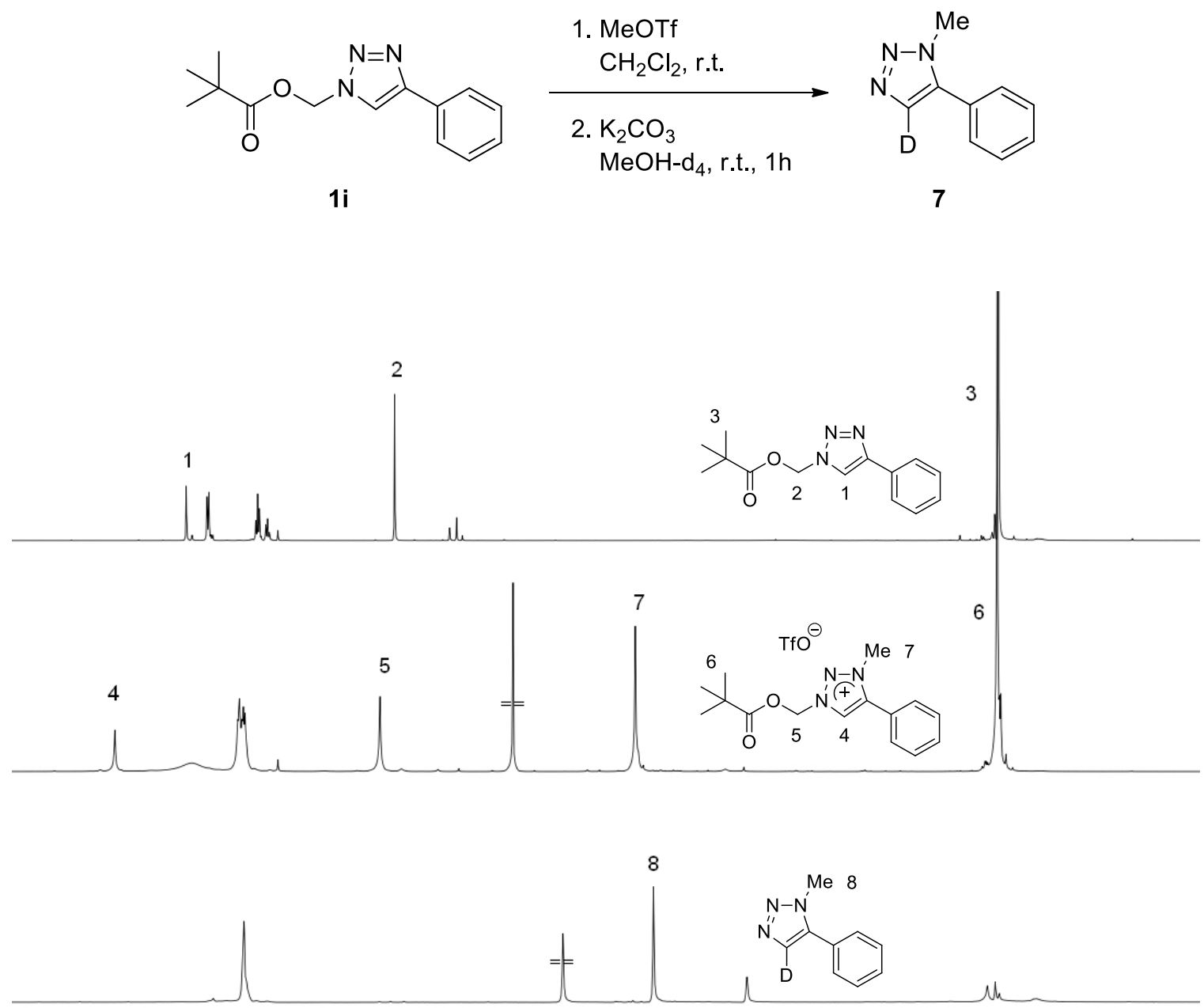

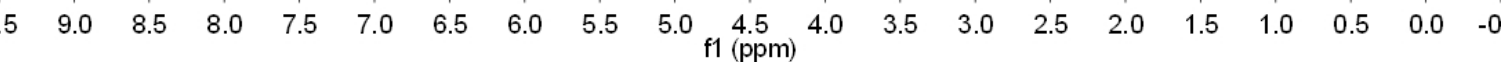

Figure S2. ${ }^{1} \mathrm{H}$ NMR $\left(400 \mathrm{MHz}, \mathrm{CDCl}_{3}\right)$ spectra of the $\mathrm{N}$-methylation reaction of $\mathbf{1 i}$ with MeOTf followed by $\mathrm{N}$-demethylation reaction in the presence of $\mathrm{K}_{2} \mathrm{CO}_{3}$ and $\mathrm{MeOH}-\mathrm{d}_{4}$. 


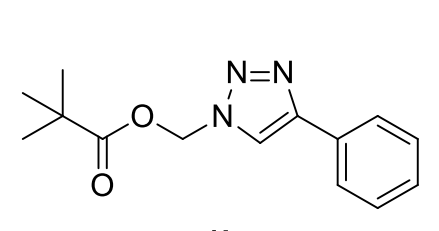

$1 \mathbf{i}$

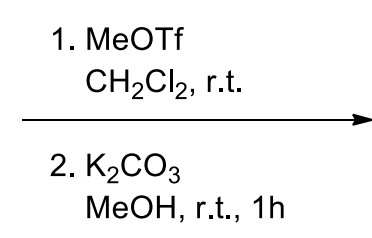

$\mathrm{MeOH}$, r.t., $1 \mathrm{~h}$

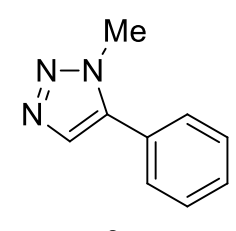

$6 a$
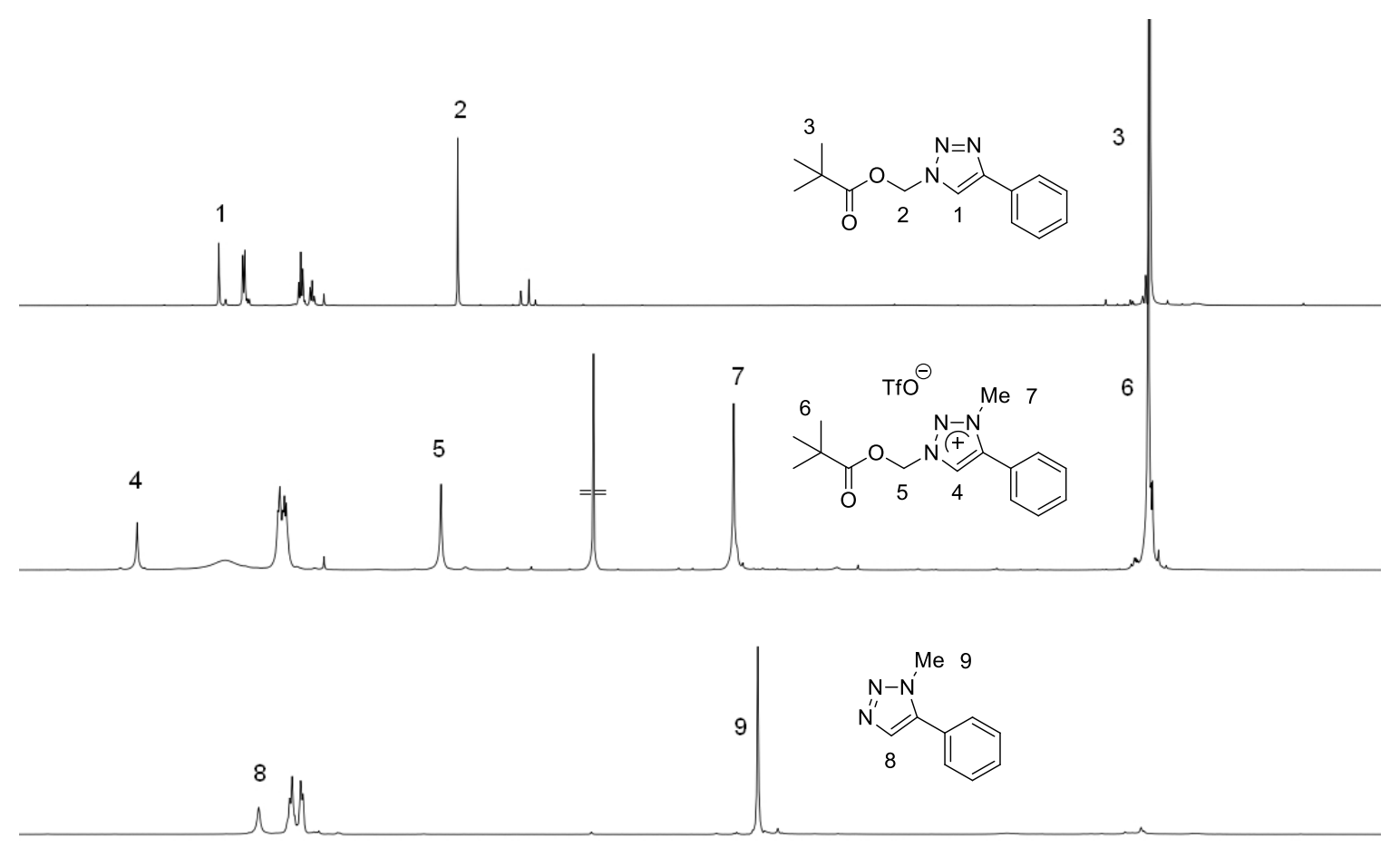

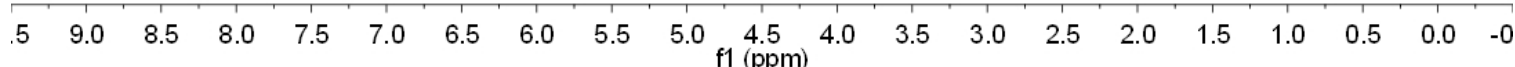

Figure S3. ${ }^{1} \mathrm{H}$ NMR $\left(400 \mathrm{MHz}, \mathrm{CDCl}_{3}\right)$ spectra of the $\mathrm{N}$-methylation reaction of $\mathbf{1 i}$ with MeOTf followed by $\mathrm{N}$-demethylation reaction in the presence of $\mathrm{K}_{2} \mathrm{CO}_{3}$ and $\mathrm{MeOH}$. 

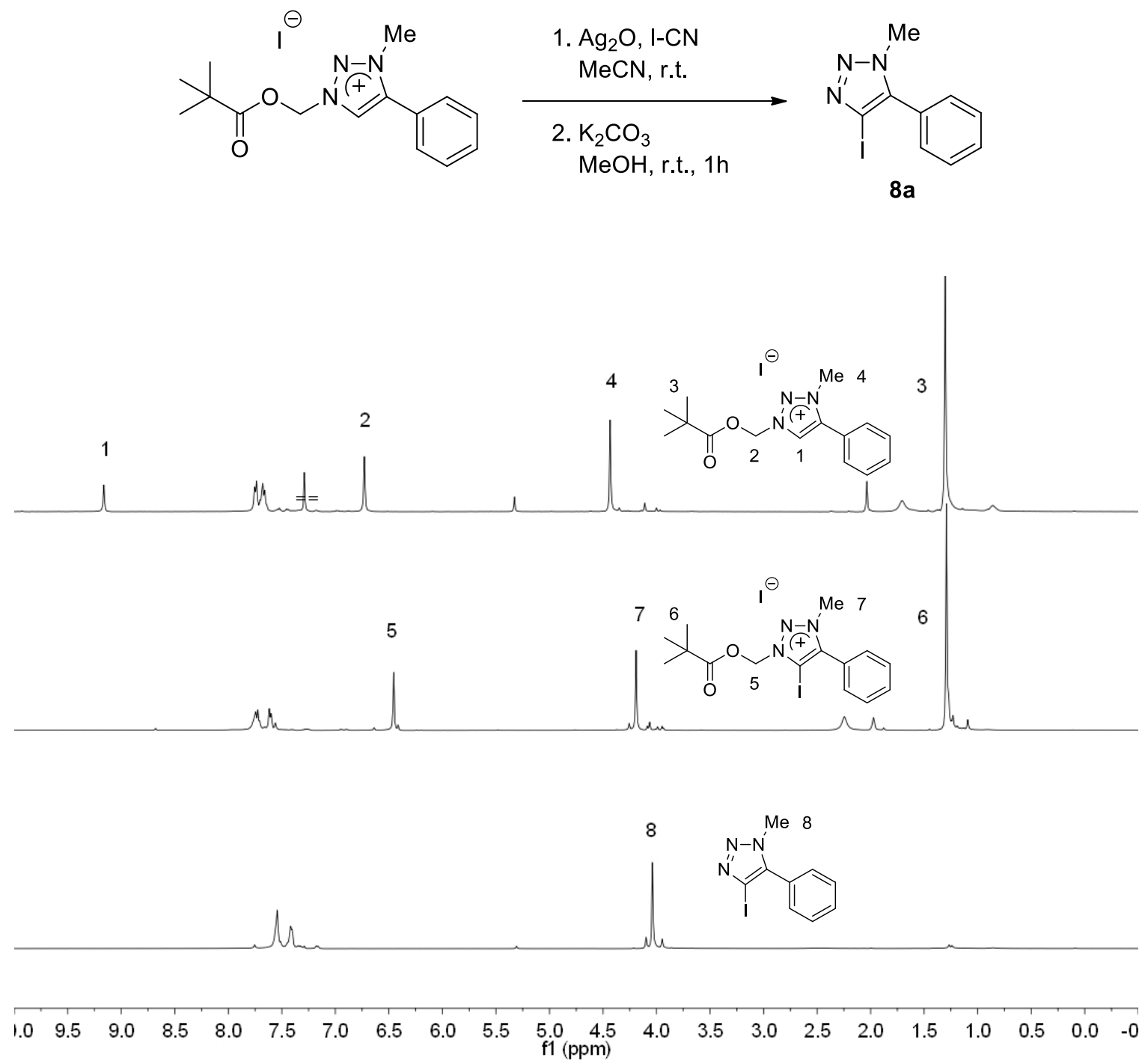

Figure S4. ${ }^{1} \mathrm{H}$ NMR (400 $\mathrm{MHz}, \mathrm{CDCl}_{3}$ and $\left.\mathrm{MeCN}-\mathrm{d}_{3}\right)$ spectra of the $\mathrm{C} 5$-iodination reaction of 1-pivaloyloxymethyl-1,2,3-triazolium iodide with $\mathrm{Ag}_{2} \mathrm{O}$ and cyanogen iodide followed by $\mathrm{N}$-demethylation reaction in the presence of $\mathrm{K}_{2} \mathrm{CO}_{3}$ and $\mathrm{MeOH}$. 


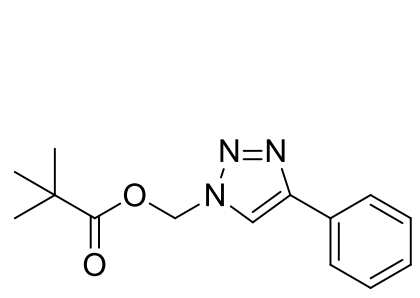

$1 \mathbf{i}$
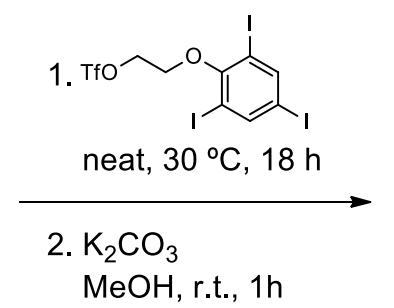

$\mathrm{MeOH}$, r.t., $1 \mathrm{~h}$

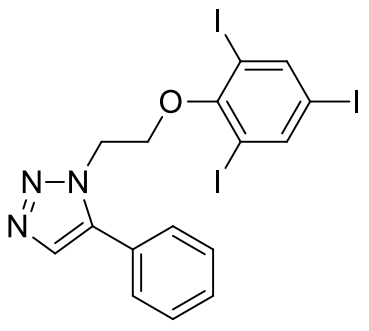

6c

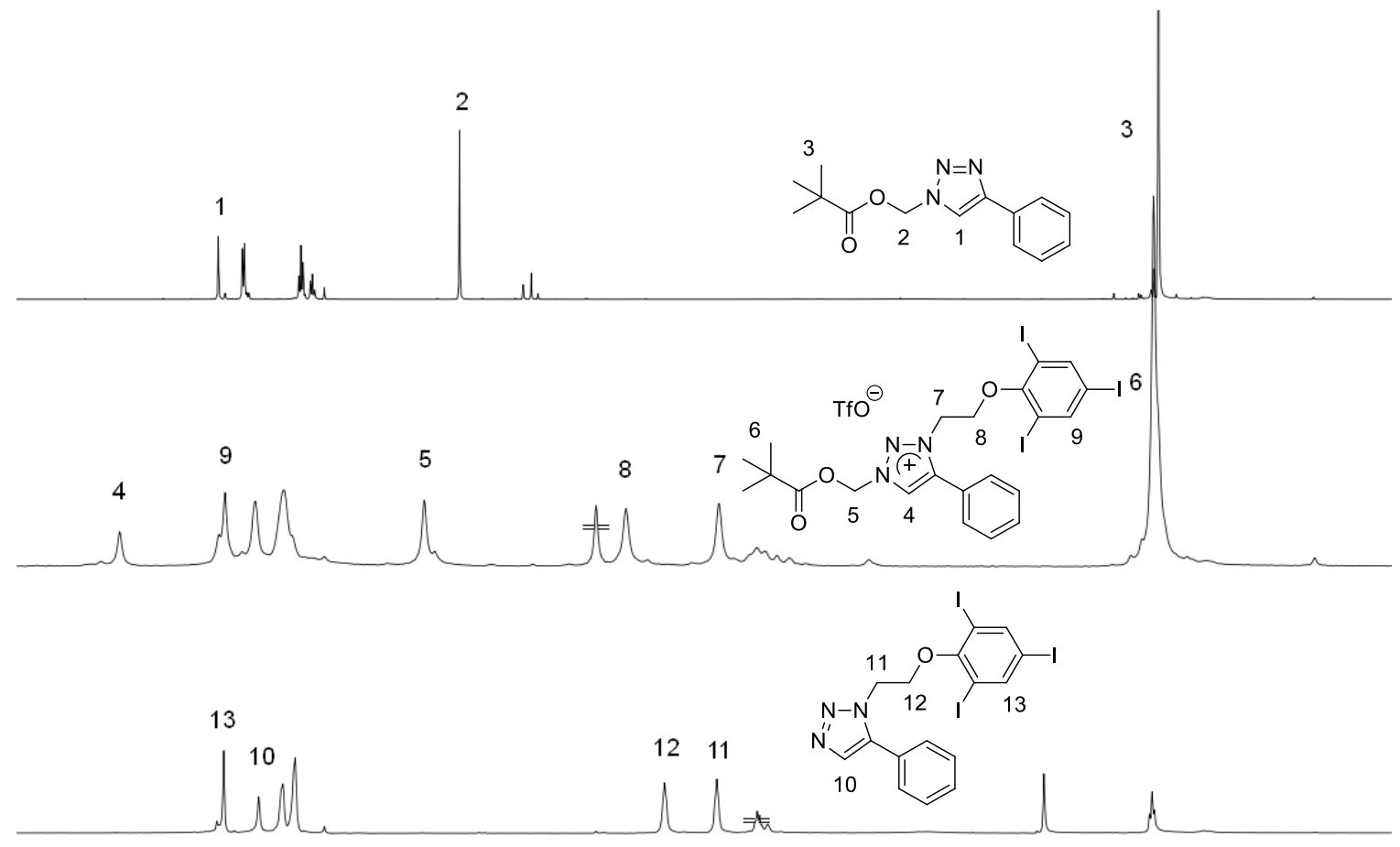

5

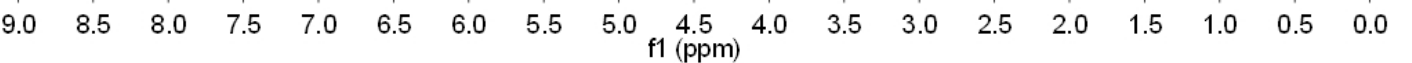

Figure S5. ${ }^{1} \mathrm{H}$ NMR ( $\left.400 \mathrm{MHz}, \mathrm{CDCl}_{3}\right)$ spectra of the N-methylation reaction of $\mathbf{1 i}$ with 2(2,4,6-triiodophenoxy)ethyl trifluoromethanesulfonate followed by $\mathrm{N}$-demethylation reaction in the presence of $\mathrm{K}_{2} \mathrm{CO}_{3}$ and $\mathrm{MeOH}$. 


\section{Computational calculations}

\subsection{Computational methods}

Density functional theory calculations were performed by use of Gamess 5 DEC 2014 (R1)

from Iowa State University suite of programs. ${ }^{20}$ Full geometry optimizations and harmonic analyses were carried out by use of the B3LYP hybrid functional [39] and the 6-31G* basis set. Solvent effects were computed by the C-PCM solvation model $(\mathrm{MeCN}, \varepsilon=$ 35.68) $(\mathrm{MeOH} \varepsilon=32.63)$ and Grimme empirical dipersion DFTD3 v1.2 ${ }^{21}$ Free energies were computed at $298.15 \mathrm{~K}$. The charges of compound D,E were obtained using NBO6.0 software. ${ }^{22}$

20 Schmidt, M.W.; Baldridge, K.K.; Boatz, J.A.; Elbert, S.T.; Gordon, M.S.; Jensen, J.H.; Koseki, S.; Matsunaga, N.; Nguyen, K.A.; Su, S.J.; Windus T.L.; Together with Dupuis, M.; Montgomery J.A. J.Comput.Chem. 1993, 14, 1347-1363.

${ }^{21}$ Grimme, S.; Antony, J.; Ehrlich S.; Krieg, H. J. Chem. Phys, 2010, 132, 154104.

22 NBO 6.0. Glendening, E. D.; Badenhoop, J. K.; Reed, A. E.; Carpenter, J. E.; Bohmann, J. A.; Morales, C. M.; Landis, C. R.; Weinhold F. Theoretical Chemistry Institute, University of Wisconsin, Madison, WI, 2013. 


\subsection{Electronic energies and cartesian coordinates for structures $D$ and $E$ NBO charges}

D

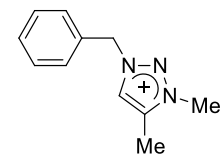

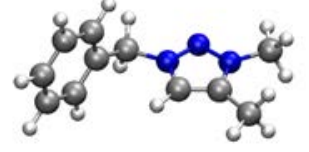

$E(R-B 3 L Y P)=-591.2669181178$

$\begin{array}{lrrr}\mathrm{N} & -6.47899 & 2.57030 & -0.77997 \\ \mathrm{~N} & -5.58395 & 2.09033 & -1.61523 \\ \mathrm{C} & -3.39014 & 1.10490 & -1.45108 \\ \mathrm{C} & -4.82644 & 1.90925 & 0.48880 \\ \mathrm{C} & -3.86490 & 1.56021 & 1.57293 \\ \mathrm{H} & -3.63698 & 0.48820 & 1.56922 \\ \mathrm{H} & -4.29066 & 1.81573 & 2.54558 \\ \mathrm{H} & -2.92255 & 2.10796 & 1.45688 \\ \mathrm{C} & -6.07723 & 2.48893 & 0.51600 \\ \mathrm{H} & -6.68609 & 2.84708 & 1.33134 \\ \mathrm{C} & -7.78392 & 3.07647 & -1.31495 \\ \mathrm{C} & -8.41907 & 4.05382 & -0.36380 \\ \mathrm{H} & -8.40888 & 2.19444 & -1.48086 \\ \mathrm{H} & -7.54307 & 3.52021 & -2.28344 \\ \mathrm{~N} & -4.58704 & 1.69250 & -0.84124 \\ \mathrm{H} & -3.53927 & 1.09300 & -2.53022 \\ \mathrm{H} & -3.25810 & 0.08724 & -1.07646 \\ \mathrm{H} & -2.51980 & 1.71420 & -1.19646 \\ \mathrm{C} & -8.00907 & 5.39450 & -0.35666 \\ \mathrm{C} & -8.57573 & 6.29365 & 0.54542 \\ \mathrm{C} & -9.55329 & 5.85809 & 1.44552 \\ \mathrm{C} & -9.40007 & 3.62190 & 0.53866 \\ \mathrm{C} & -9.96636 & 4.52389 & 1.44150 \\ \mathrm{H} & -7.25477 & 5.73546 & -1.06258 \\ \mathrm{H} & -8.26308 & 7.33369 & 0.54105 \\ \mathrm{H} & -9.99797 & 6.56148 & 2.14351 \\ \mathrm{H} & -9.73193 & 2.58575 & 0.52585 \\ \mathrm{H} & -10.73419 & 4.18714 & 2.13172\end{array}$

$\mathbf{E}$

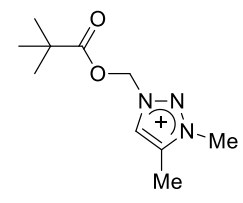

$E(R-B 3 L Y P)=-705.9832816735$

$\begin{array}{lccc}\mathrm{C} & -4.82713 & 1.02890 & 2.93302 \\ \mathrm{~N} & -3.88993 & 0.63975 & 1.87355 \\ \mathrm{H} & -4.79798 & 2.11380 & 3.05457 \\ \mathrm{H} & -4.50982 & 0.53801 & 3.85241 \\ \mathrm{H} & -5.83458 & 0.70703 & 2.65913 \\ \mathrm{C} & -3.86061 & 1.08514 & 0.57782 \\ \mathrm{C} & -4.81754 & 2.08477 & 0.02471 \\ \mathrm{H} & -5.85175 & 1.73386 & 0.11502 \\ \mathrm{H} & -4.60664 & 2.25998 & -1.03229 \\ \mathrm{H} & -4.73542 & 3.04140 & 0.55371 \\ \mathrm{C} & -2.79720 & 0.41295 & 0.01425 \\ \mathrm{~N} & -2.28718 & -0.36328 & 1.00618 \\ \mathrm{~N} & -2.93627 & -0.23452 & 2.13982 \\ \mathrm{H} & -2.37138 & 0.44081 & -0.97677 \\ \mathrm{C} & -1.12577 & -1.29449 & 0.92420 \\ \mathrm{O} & -0.18242 & -0.78816 & 0.03221 \\ \mathrm{H} & -1.48028 & -2.24363 & 0.52025 \\ \mathrm{H} & -0.75400 & -1.39681 & 1.94541 \\ \mathrm{C} & 0.60261 & 0.26840 & 0.53454 \\ \mathrm{O} & 0.36045 & 0.73185 & 1.61857 \\ \mathrm{C} & 1.68129 & 0.67297 & -0.45327 \\ \mathrm{C} & 1.00926 & 1.08104 & -1.78559 \\ \mathrm{C} & 2.61788 & -0.53730 & -0.68253 \\ \mathrm{C} & 2.46429 & 1.85568 & 0.13906 \\ \mathrm{H} & 0.45562 & 0.24776 & -2.22937 \\ \mathrm{H} & 1.78191 & 1.39184 & -2.49602 \\ \mathrm{H} & 0.32676 & 1.92848 & -1.64503 \\ \mathrm{H} & 1.81271 & 2.71694 & 0.31723 \\ \mathrm{H} & 3.25181 & 2.15455 & -0.55985 \\ \mathrm{H} & 2.93137 & 1.58499 & 1.09064 \\ \mathrm{H} & 2.08015 & -1.38870 & -1.11008 \\ \mathrm{H} & 3.09106 & -0.85435 & 0.25345 \\ \mathrm{H} & 3.41192 & -0.24757 & -1.37843\end{array}$


5.3. Comparison of the free energy profiles of the hydroxide-promoted N-dealkylation of a 1,2,3-triazole and 1,2,3-triazolium salt comprising pivaloyloxymethyl group. Electronic energies and cartesian coordinates.
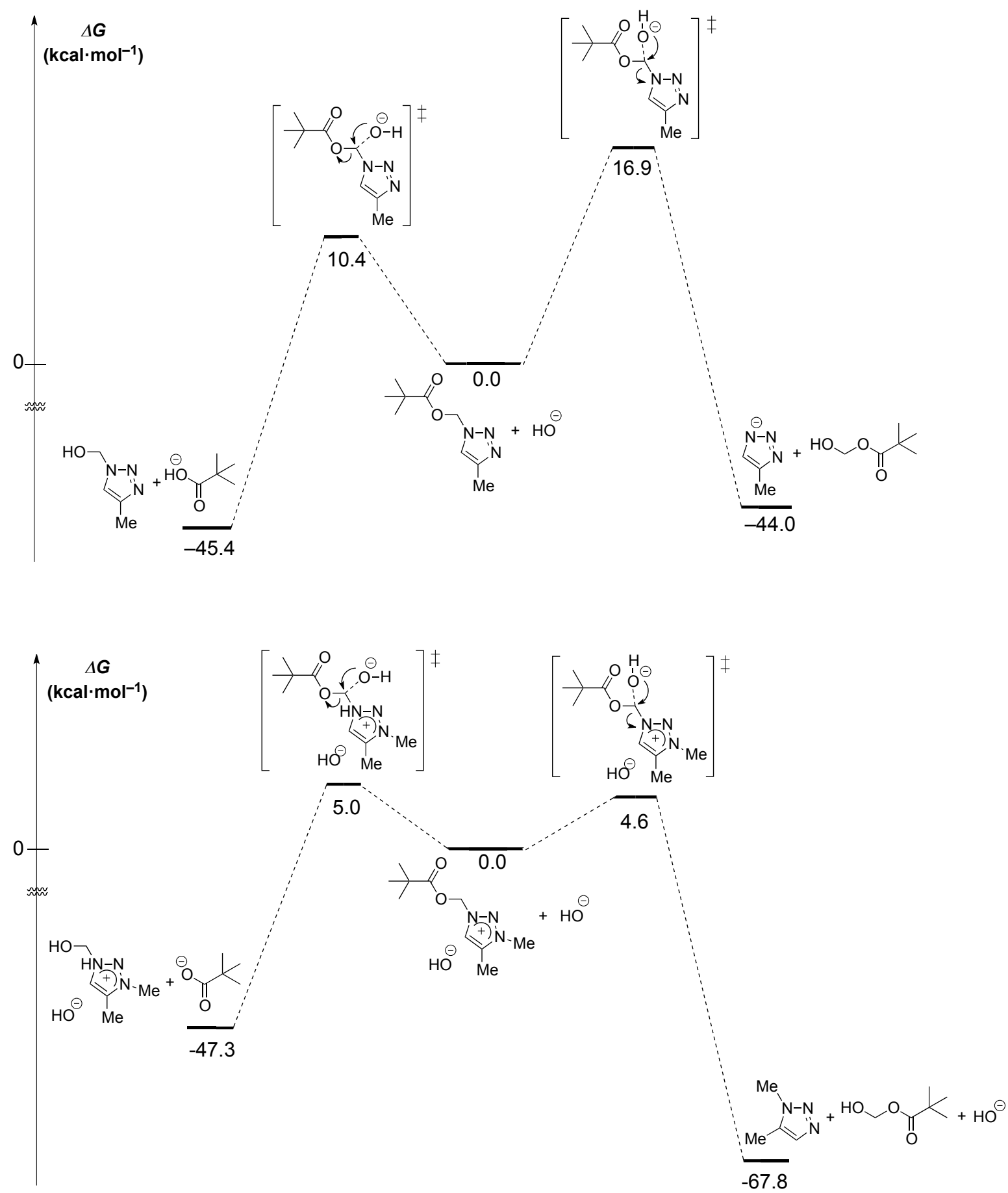

Figure S6. Transition states and reaction products computed at B3LYP/6-31G* level of theory for the nucleophilic N-dealkylation of 1,2,3-triazole (above) and 1,2,3-triazolium salt (below). Relative $\Delta G^{\ddagger}$ energies in $\mathrm{kcal} \cdot \mathrm{mol}^{-1}$ calculated at $25^{\circ} \mathrm{C}$ in $\mathrm{MeOH}$. 

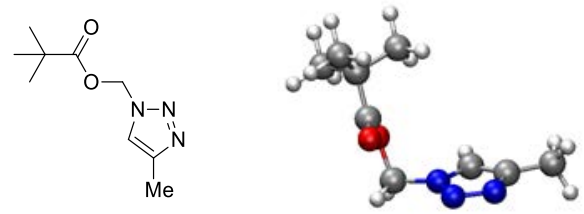

$\mathrm{E}(\mathrm{R}-\mathrm{B} 3 \mathrm{LYP})=-666.3245292$

$\begin{array}{lccc}\mathrm{N} & -3.61447 & 1.15351 & 1.68237 \\ \mathrm{C} & -3.94243 & 0.90750 & 0.37252 \\ \mathrm{C} & -5.06986 & 1.61110 & -0.31456 \\ \mathrm{H} & -6.04014 & 1.27244 & 0.06852 \\ \mathrm{H} & -5.04653 & 1.42411 & -1.39194 \\ \mathrm{H} & -5.00920 & 2.69240 & -0.14985 \\ \mathrm{C} & -3.08065 & -0.05673 & -0.10129 \\ \mathrm{~N} & -2.27242 & -0.34618 & 0.95426 \\ \mathrm{~N} & -2.60332 & 0.40476 & 2.03076 \\ \mathrm{H} & -2.97846 & -0.54401 & -1.05896 \\ \mathrm{C} & -1.14052 & -1.24636 & 0.99429 \\ \mathrm{O} & -0.08555 & -0.76819 & 0.15909 \\ \mathrm{H} & -1.42347 & -2.21522 & 0.58558 \\ \mathrm{H} & -0.82501 & -1.32960 & 2.03377 \\ \mathrm{C} & 0.74815 & 0.17607 & 0.69694 \\ \mathrm{O} & 0.65782 & 0.54120 & 1.84876 \\ \mathrm{C} & 1.73644 & 0.70036 & -0.34036 \\ \mathrm{C} & 0.93120 & 1.53016 & -1.36876 \\ \mathrm{C} & 2.43955 & -0.47722 & -1.04954 \\ \mathrm{C} & 2.76936 & 1.59031 & 0.36944 \\ \mathrm{H} & 0.18173 & 0.91396 & -1.87522 \\ \mathrm{H} & 1.61639 & 1.93377 & -2.12233 \\ \mathrm{H} & 0.42187 & 2.37147 & -0.88472 \\ \mathrm{H} & 2.28504 & 2.43328 & 0.87133 \\ \mathrm{H} & 3.48153 & 1.98382 & -0.36384 \\ \mathrm{H} & 3.32586 & 1.02160 & 1.12186 \\ \mathrm{H} & 1.72287 & -1.10960 & -1.58074 \\ \mathrm{H} & 2.98668 & -1.10057 & -0.33283 \\ \mathrm{H} & 3.15871 & -0.08323 & -1.77599\end{array}$

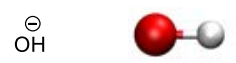

$\mathrm{E}(\mathrm{R}-\mathrm{B} 3 \mathrm{LYP})=-75.8256250$

$\begin{array}{llll}\text { O } & -6.46996 & 2.62939 & 0.00000\end{array}$ $\begin{array}{llll}\mathrm{H} & -5.49344 & 2.62939 & 0.00000\end{array}$
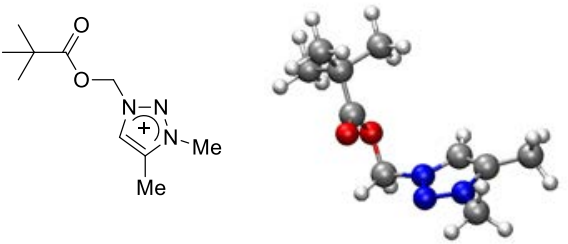

$E(R-B 3 L Y P)=-706.0548936$

$\begin{array}{lccc}\mathrm{C} & -4.44729 & 1.42405 & 2.90879 \\ \mathrm{~N} & -3.74346 & 0.75165 & 1.81261 \\ \mathrm{H} & -4.23541 & 2.49442 & 2.85661 \\ \mathrm{H} & -4.07852 & 1.01091 & 3.84619 \\ \mathrm{H} & -5.51923 & 1.24621 & 2.80772 \\ \mathrm{C} & -3.96565 & 0.90804 & 0.46951 \\ \mathrm{C} & -5.02890 & 1.78291 & -0.09499 \\ \mathrm{H} & -6.01645 & 1.48874 & 0.27646 \\ \mathrm{H} & -5.03209 & 1.70446 & -1.18343 \\ \mathrm{H} & -4.85594 & 2.82973 & 0.17906 \\ \mathrm{C} & -3.01111 & 0.11392 & -0.12595 \\ \mathrm{~N} & -2.31166 & -0.44669 & 0.89566 \\ \mathrm{~N} & -2.74267 & -0.06209 & 2.08046 \\ \mathrm{H} & -2.78680 & -0.06388 & -1.16588 \\ \mathrm{C} & -1.17336 & -1.38007 & 0.79890 \\ \mathrm{O} & -0.15950 & -0.81174 & 0.00409 \\ \mathrm{H} & -1.50834 & -2.27927 & 0.28596 \\ \mathrm{H} & -0.85308 & -1.58739 & 1.81971 \\ \mathrm{C} & 0.62549 & 0.15077 & 0.61490 \\ \mathrm{O} & 0.44008 & 0.47569 & 1.76412 \\ \mathrm{C} & 1.67292 & 0.69765 & -0.34508 \\ \mathrm{C} & 0.95018 & 1.29289 & -1.57576 \\ \mathrm{C} & 2.59930 & -0.45850 & -0.78583 \\ \mathrm{C} & 2.48095 & 1.78583 & 0.37911 \\ \mathrm{H} & 0.39434 & 0.52511 & -2.12167 \\ \mathrm{H} & 1.69299 & 1.72719 & -2.25370 \\ \mathrm{H} & 0.25541 & 2.08811 & -1.28174 \\ \mathrm{H} & 1.83155 & 2.59854 & 0.71972 \\ \mathrm{H} & 3.22684 & 2.20221 & -0.30604 \\ \mathrm{H} & 3.00133 & 1.37699 & 1.25107 \\ \mathrm{H} & 2.04014 & -1.24224 & -1.30526 \\ \mathrm{H} & 3.11045 & -0.90641 & 0.07389 \\ \mathrm{H} & 3.36067 & -0.06565 & -1.46869\end{array}$




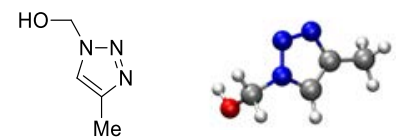

$E(R-B 3 L Y P)=-395.8693148$

$\begin{array}{lrrr}\mathrm{N} & -3.52800 & 0.90638 & 1.87401 \\ \mathrm{C} & -3.85657 & 0.85272 & 0.54402 \\ \mathrm{C} & -4.93113 & 1.70737 & -0.05106 \\ \mathrm{H} & -5.91839 & 1.43181 & 0.33867 \\ \mathrm{H} & -4.95377 & 1.60035 & -1.13939 \\ \mathrm{H} & -4.76586 & 2.76402 & 0.18775 \\ \mathrm{C} & -3.04633 & -0.09140 & -0.05104 \\ \mathrm{~N} & -2.27133 & -0.56833 & 0.95692 \\ \mathrm{~N} & -2.56603 & 0.05412 & 2.11937 \\ \mathrm{H} & -2.96325 & -0.44897 & -1.06631 \\ \mathrm{C} & -1.15900 & -1.51408 & 0.88944 \\ \mathrm{O} & -0.01363 & -0.95527 & 0.30064 \\ \mathrm{H} & -1.46134 & -2.35383 & 0.26134 \\ \mathrm{H} & -0.99553 & -1.85539 & 1.91677 \\ \mathrm{H} & 0.35598 & -0.30174 & 0.91838\end{array}$

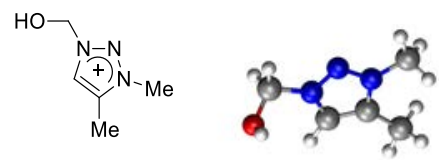

$E(R-B 3 L Y P)=-435.6024226$

$\begin{array}{lrrr}\mathrm{C} & -4.56044 & 1.30706 & 2.96381 \\ \mathrm{~N} & -3.76700 & 0.67755 & 1.90367 \\ \mathrm{H} & -4.47477 & 2.39078 & 2.86902 \\ \mathrm{H} & -4.15525 & 0.97898 & 3.91935 \\ \mathrm{H} & -5.60220 & 0.99501 & 2.86177 \\ \mathrm{C} & -3.91180 & 0.84224 & 0.55268 \\ \mathrm{C} & -4.92635 & 1.73797 & -0.06704 \\ \mathrm{H} & -5.93787 & 1.47245 & 0.25852 \\ \mathrm{H} & -4.87778 & 1.65445 & -1.15422 \\ \mathrm{H} & -4.74048 & 2.78188 & 0.20988 \\ \mathrm{C} & -2.92977 & 0.04507 & 0.00622 \\ \mathrm{~N} & -2.29154 & -0.52408 & 1.05982 \\ \mathrm{~N} & -2.78656 & -0.14939 & 2.21969 \\ \mathrm{H} & -2.64478 & -0.13562 & -1.01785 \\ \mathrm{C} & -1.15022 & -1.49053 & 1.00459 \\ \mathrm{O} & -0.22247 & -1.08314 & 0.06131 \\ \mathrm{H} & -1.56155 & -2.44690 & 0.67772 \\ \mathrm{H} & -0.77862 & -1.56128 & 2.03041 \\ \mathrm{H} & 0.31934 & -0.36279 & 0.42695\end{array}$

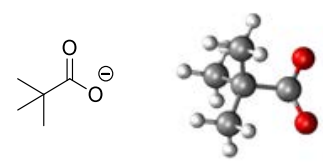

$E(R-B 3 L Y P)=-346.3516447$

$\begin{array}{lrrr}\mathrm{C} & -3.43321 & 1.02169 & -0.03115 \\ \mathrm{C} & -1.89356 & 0.99968 & -0.03257 \\ \mathrm{C} & -3.91898 & 2.12996 & 0.92152 \\ \mathrm{C} & -3.97164 & -0.33788 & 0.43133 \\ \mathrm{C} & -3.93344 & 1.37269 & -1.47884 \\ \mathrm{H} & -3.57523 & 1.94985 & 1.95010 \\ \mathrm{H} & -5.01638 & 2.17946 & 0.94023 \\ \mathrm{H} & -3.54167 & 3.10174 & 0.58877 \\ \mathrm{H} & -1.49711 & 0.82134 & 0.97730 \\ \mathrm{H} & -1.50548 & 1.95449 & -0.40022 \\ \mathrm{H} & -1.51129 & 0.20339 & -0.68555 \\ \mathrm{H} & -3.61350 & -0.58312 & 1.44145 \\ \mathrm{H} & -3.65338 & -1.13066 & -0.25373 \\ \mathrm{H} & -5.06668 & -0.33893 & 0.44070 \\ \mathrm{O} & -4.70135 & 0.54877 & -2.04592 \\ \mathrm{O} & -3.52873 & 2.47474 & -1.94915\end{array}$

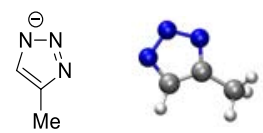

$E(R-B 3 L Y P)=-280.9080632$

$\begin{array}{lccc}\mathrm{C} & -8.03373 & 1.16105 & -0.04309 \\ \mathrm{C} & -6.74744 & 0.68841 & 0.20185 \\ \mathrm{C} & -9.34640 & 0.43119 & -0.02589 \\ \mathrm{H} & -9.76437 & 0.29747 & -1.03412 \\ \mathrm{H} & -10.10230 & 0.96855 & 0.56179 \\ \mathrm{H} & -9.23091 & -0.56662 & 0.41318 \\ \mathrm{~N} & -7.92439 & 2.48157 & -0.33173 \\ \mathrm{~N} & -6.61562 & 2.79844 & -0.25960 \\ \mathrm{~N} & -5.87883 & 1.71987 & 0.06056 \\ \mathrm{H} & -6.41881 & -0.31162 & 0.46556\end{array}$




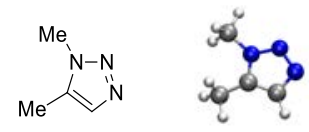

$E(R-B 3 L Y P)=-320.6871625$

$\begin{array}{lrrr}\mathrm{C} & -8.02575 & 1.15772 & 0.07805 \\ \mathrm{C} & -6.73160 & 0.67121 & 0.09182 \\ \mathrm{C} & -9.35712 & 0.50156 & 0.23026 \\ \mathrm{H} & -9.98632 & 0.65762 & -0.65464 \\ \mathrm{H} & -9.90242 & 0.89415 & 1.09753 \\ \mathrm{H} & -9.22758 & -0.57455 & 0.36984 \\ \mathrm{~N} & -7.85948 & 2.49224 & -0.12226 \\ \mathrm{~N} & -6.54881 & 2.81361 & -0.22723 \\ \mathrm{~N} & -5.86385 & 1.70315 & -0.09589 \\ \mathrm{H} & -6.39464 & -0.34781 & 0.22380 \\ \mathrm{C} & -8.88824 & 3.51617 & -0.22786 \\ \mathrm{H} & -9.52098 & 3.32780 & -1.09997 \\ \mathrm{H} & -8.38955 & 4.47931 & -0.33824 \\ \mathrm{H} & -9.50542 & 3.52260 & 0.67478\end{array}$

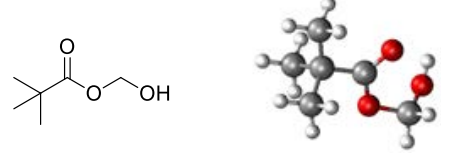

$\begin{array}{lccc}\text { E(R-B3LYP) }=-461.3095115 & \\ \text { O } & -4.90632 & 0.35947 & -0.14724 \\ \mathrm{C} & -4.83780 & 1.59135 & 0.49083 \\ \mathrm{O} & -3.51795 & 2.19555 & 0.39692 \\ \mathrm{H} & -5.09854 & 1.53340 & 1.55316 \\ \mathrm{H} & -5.48894 & 2.28688 & -0.03754 \\ \mathrm{C} & -2.54622 & 1.56311 & 1.08952 \\ \mathrm{O} & -2.75122 & 0.52378 & 1.69712 \\ \mathrm{C} & -1.20628 & 2.29268 & 1.00877 \\ \mathrm{C} & -1.38134 & 3.71187 & 1.59456 \\ \mathrm{C} & -0.78501 & 2.38587 & -0.47502 \\ \mathrm{C} & -0.15846 & 1.50960 & 1.81301 \\ \mathrm{H} & -2.12143 & 4.28497 & 1.02859 \\ \mathrm{H} & -0.42464 & 4.24560 & 1.55596 \\ \mathrm{H} & -1.70328 & 3.67010 & 2.64189 \\ \mathrm{H} & -0.43765 & 1.44842 & 2.86985 \\ \mathrm{H} & 0.81118 & 2.01478 & 1.74148 \\ \mathrm{H} & -0.04736 & 0.48946 & 1.43200 \\ \mathrm{H} & -1.52220 & 2.94198 & -1.06135 \\ \mathrm{H} & -0.67070 & 1.38939 & -0.91794 \\ \mathrm{H} & 0.17892 & 2.90245 & -0.54915 \\ \mathrm{H} & -4.42910 & -0.27002 & 0.42288\end{array}$

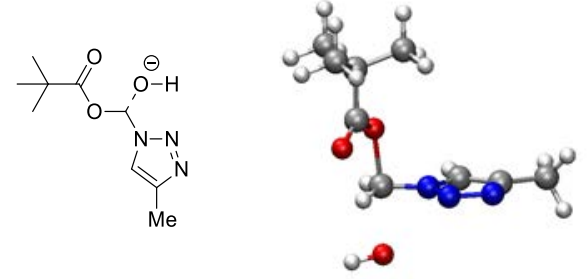

$\mathrm{E}(\mathrm{R}-\mathrm{B} 3 \mathrm{~L} Y \mathrm{P})=-742.1496967$

Freq $=-564.970$

$\begin{array}{lrrr}\mathrm{N} & -0.35275 & 2.09125 & -1.65515 \\ \mathrm{C} & -0.93499 & 1.47305 & -2.72832 \\ \mathrm{C} & -2.14591 & 2.02047 & -3.41807 \\ \mathrm{H} & -3.04178 & 1.92231 & -2.79225 \\ \mathrm{H} & -2.33034 & 1.48703 & -4.35538 \\ \mathrm{H} & -2.01990 & 3.08479 & -3.64658 \\ \mathrm{C} & -0.21621 & 0.32474 & -2.99240 \\ \mathrm{~N} & 0.75980 & 0.30514 & -2.05478 \\ \mathrm{~N} & 0.67686 & 1.38094 & -1.25236 \\ \mathrm{H} & -0.31505 & -0.44647 & -3.74036 \\ \mathrm{C} & 1.80677 & -0.63270 & -1.90024 \\ \mathrm{O} & 2.97895 & 0.13847 & -2.96170 \\ \mathrm{H} & 1.73083 & -1.53647 & -2.47129 \\ \mathrm{H} & 2.35756 & -0.56857 & -0.98162 \\ \mathrm{C} & 4.00731 & 0.70755 & -2.38347 \\ \mathrm{O} & 4.26377 & 0.65776 & -1.17679 \\ \mathrm{C} & 4.91714 & 1.46207 & -3.38294 \\ \mathrm{C} & 4.07642 & 2.52962 & -4.11363 \\ \mathrm{C} & 5.46512 & 0.44543 & -4.40641 \\ \mathrm{C} & 6.07657 & 2.13130 & -2.63133 \\ \mathrm{H} & 3.23622 & 2.06876 & -4.64004 \\ \mathrm{H} & 4.69619 & 3.06393 & -4.84465 \\ \mathrm{H} & 3.67709 & 3.26691 & -3.40661 \\ \mathrm{H} & 5.70188 & 2.84628 & -1.89195 \\ \mathrm{H} & 6.72283 & 2.66671 & -3.33777 \\ \mathrm{H} & 6.68108 & 1.38928 & -2.10040 \\ \mathrm{H} & 4.64700 & -0.05473 & -4.93339 \\ \mathrm{H} & 6.09654 & 0.95399 & -5.14563 \\ \mathrm{O} & 0.45386 & -1.84423 & -0.73324 \\ \mathrm{H} & 6.07450 & -0.32169 & -3.91243 \\ \mathrm{H} & 1.13375 & -2.37718 & -0.28288\end{array}$



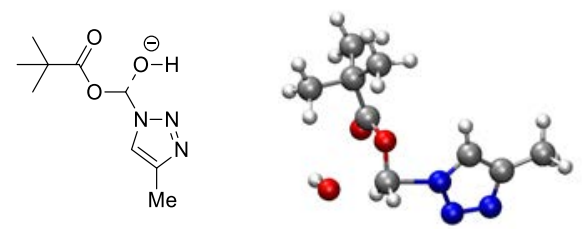

$\mathrm{E}(\mathrm{R}-\mathrm{B} 3 \mathrm{~L} Y \mathrm{P})=-742.1393969$

Freq $=-584.592$

$\begin{array}{lrrr}\mathrm{O} & -1.65442 & 2.73367 & 0.65321 \\ \mathrm{~N} & 1.65517 & 0.68671 & 0.24845 \\ \mathrm{C} & 0.02627 & 1.55272 & 0.46757 \\ \mathrm{C} & 3.37190 & -0.65775 & 0.11352 \\ \mathrm{C} & 4.32845 & -1.81127 & 0.18329 \\ \mathrm{H} & 4.91835 & -1.88795 & -0.73749 \\ \mathrm{H} & 3.79267 & -2.75605 & 0.32429 \\ \mathrm{H} & 5.03689 & -1.70564 & 1.01593 \\ \mathrm{C} & 2.05135 & -0.57202 & 0.53551 \\ \mathrm{~N} & 2.65577 & 1.35753 & -0.32002 \\ \mathrm{H} & 1.39136 & -1.29357 & 0.99510 \\ \mathrm{C} & -1.41250 & -0.08438 & -0.39079 \\ \mathrm{O} & -1.18096 & 0.24535 & -1.53710 \\ \mathrm{C} & -2.57330 & -0.96931 & 0.04889 \\ \mathrm{C} & -3.74659 & 0.03739 & 0.21375 \\ \mathrm{C} & -2.29393 & -1.67660 & 1.38665 \\ \mathrm{C} & -2.89133 & -1.99870 & -1.04620 \\ \mathrm{H} & -3.40514 & 0.92646 & 0.75784 \\ \mathrm{H} & -4.57613 & -0.43881 & 0.74896 \\ \mathrm{H} & -4.10835 & 0.36446 & -0.76786 \\ \mathrm{H} & -3.02594 & -1.50828 & -2.01444 \\ \mathrm{H} & -3.81413 & -2.53281 & -0.79362 \\ \mathrm{H} & -2.08516 & -2.73589 & -1.14303 \\ \mathrm{H} & -2.15780 & -0.95898 & 2.19952 \\ \mathrm{H} & -3.14241 & -2.32422 & 1.63643 \\ \mathrm{H} & -1.39571 & -2.30264 & 1.32650 \\ \mathrm{H} & -2.01446 & 2.60706 & -0.24064 \\ \mathrm{~N} & 3.71545 & 0.55006 & -0.41069 \\ \mathrm{H} & 0.31464 & 2.06812 & 1.36392 \\ \mathrm{H} & 0.09807 & 1.99741 & -0.50714 \\ \mathrm{O} & -0.73108 & 0.40570 & 0.67404\end{array}$
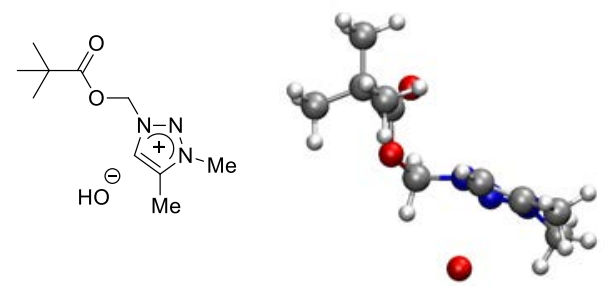

$E(R-B 3 L Y P)=-781.9102850$

$\begin{array}{lrrr}\mathrm{C} & -5.23442 & 1.01341 & 2.69764 \\ \mathrm{~N} & -3.99719 & 1.13000 & 1.93633 \\ \mathrm{H} & -5.99119 & 1.66410 & 2.26045 \\ \mathrm{H} & -5.05173 & 1.30280 & 3.73408 \\ \mathrm{H} & -5.50699 & -0.04622 & 2.62699 \\ \mathrm{C} & -3.85838 & 1.45714 & 0.60262 \\ \mathrm{C} & -4.89203 & 2.18806 & -0.18388 \\ \mathrm{H} & -5.82414 & 1.61436 & -0.24180 \\ \mathrm{H} & -4.53073 & 2.35678 & -1.20097 \\ \mathrm{H} & -5.11959 & 3.16090 & 0.26666 \\ \mathrm{C} & -2.62025 & 0.99404 & 0.25637 \\ \mathrm{~N} & -2.09222 & 0.43941 & 1.39724 \\ \mathrm{~N} & -2.98067 & 0.40487 & 2.37753 \\ \mathrm{H} & -2.07529 & 1.04037 & -0.67216 \\ \mathrm{C} & -1.08452 & -0.62397 & 1.45928 \\ \mathrm{O} & -0.13374 & -0.44315 & 0.43204 \\ \mathrm{H} & -1.64942 & -1.54616 & 1.28400 \\ \mathrm{H} & -0.63107 & -0.60022 & 2.45083 \\ \mathrm{C} & 0.86894 & 0.46670 & 0.67682 \\ \mathrm{O} & 0.94031 & 1.07779 & 1.71887 \\ \mathrm{C} & 1.80938 & 0.58643 & -0.51722 \\ \mathrm{C} & 0.99335 & 1.09825 & -1.72745 \\ \mathrm{C} & 2.40608 & -0.80314 & -0.83577 \\ \mathrm{C} & 2.92497 & 1.58203 & -0.16617 \\ \mathrm{H} & 0.20509 & 0.39080 & -2.00316 \\ \mathrm{H} & 1.66013 & 1.22176 & -2.58782 \\ \mathrm{H} & 0.53417 & 2.07094 & -1.51357 \\ \mathrm{H} & 2.51381 & 2.56861 & 0.07014 \\ \mathrm{H} & 3.60737 & 1.68514 & -1.01691 \\ \mathrm{H} & 3.50019 & 1.23846 & 0.69951 \\ \mathrm{H} & 1.62312 & -1.52369 & -1.08792 \\ \mathrm{H} & 2.97653 & -1.19251 & 0.01538 \\ \mathrm{H} & 3.08673 & -0.71626 & -1.69010 \\ \mathrm{O} & -3.89608 & -1.66590 & 1.72784 \\ \mathrm{H} & -3.73674 & -1.93041 & 2.64911\end{array}$



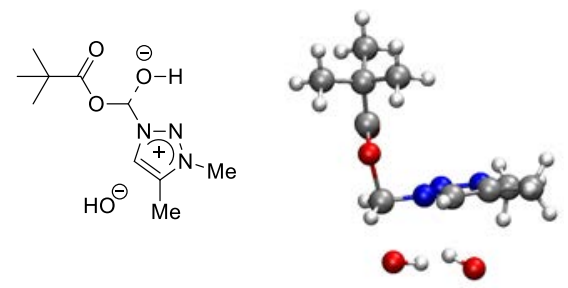

$\mathrm{E}(\mathrm{R}-\mathrm{B} 3 \mathrm{LYP})=-857.7509630$

Freq $=-569.53$

$\begin{array}{lrrr}\mathrm{C} & -3.92947 & 1.87696 & 2.67785 \\ \mathrm{~N} & -3.28536 & 1.11945 & 1.61120 \\ \mathrm{H} & -4.59647 & 2.61804 & 2.23654 \\ \mathrm{H} & -3.16096 & 2.37385 & 3.27352 \\ \mathrm{H} & -4.45975 & 1.06362 & 3.24701 \\ \mathrm{C} & -3.75295 & 0.87918 & 0.34907 \\ \mathrm{C} & -4.81732 & 1.68358 & -0.31572 \\ \mathrm{H} & -5.78759 & 1.53913 & 0.17222 \\ \mathrm{H} & -4.91105 & 1.38037 & -1.36101 \\ \mathrm{H} & -4.57808 & 2.75221 & -0.28655 \\ \mathrm{C} & -3.02790 & -0.20383 & -0.09002 \\ \mathrm{~N} & -2.21774 & -0.54917 & 0.94514 \\ \mathrm{~N} & -2.35542 & 0.24985 & 1.98228 \\ \mathrm{H} & -3.02925 & -0.73532 & -1.02733 \\ \mathrm{C} & -1.27181 & -1.61570 & 0.98156 \\ \mathrm{O} & -0.03432 & -0.87490 & 0.03083 \\ \mathrm{H} & -1.44011 & -2.41970 & 0.29128 \\ \mathrm{C} & 0.74043 & -0.00878 & 0.65050 \\ \mathrm{O} & 0.73894 & 0.18277 & 1.86630 \\ \mathrm{C} & 1.66363 & 0.76906 & -0.31351 \\ \mathrm{C} & 0.79840 & 1.41431 & -1.41612 \\ \mathrm{C} & 2.65864 & -0.22643 & -0.94669 \\ \mathrm{C} & 2.42189 & 1.85419 & 0.46359 \\ \mathrm{H} & 0.26318 & 0.65121 & -1.98741 \\ \mathrm{H} & 1.43189 & 1.98946 & -2.10266 \\ \mathrm{H} & 0.05749 & 2.09976 & -0.98561 \\ \mathrm{H} & 1.72589 & 2.56535 & 0.92127 \\ \mathrm{H} & 3.08778 & 2.40663 & -0.21073 \\ \mathrm{H} & 3.02545 & 1.41578 & 1.26441 \\ \mathrm{H} & 2.12975 & -1.01909 & -1.48484 \\ \mathrm{H} & 3.28917 & -0.69284 & -0.17967 \\ \mathrm{H} & 3.31614 & 0.29520 & -1.65337 \\ \mathrm{O} & -4.79002 & -0.77999 & 3.46916 \\ \mathrm{H} & -3.86111 & -0.93693 & 3.70348 \\ \mathrm{O} & -2.79651 & -2.70420 & 1.95647 \\ \mathrm{H} & -3.47427 & -2.06964 & 2.26145 \\ \mathrm{H} & -0.76966 & -1.75116 & 1.91891 \\ & & & \\ & & & \end{array}$

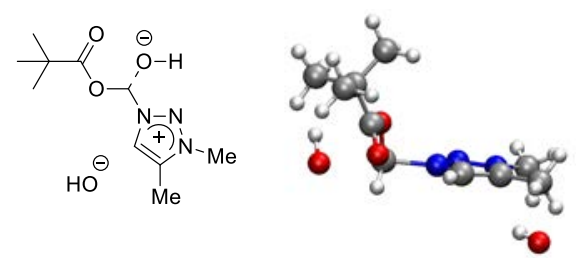

$\mathrm{E}(\mathrm{R}-\mathrm{B} 3 \mathrm{~L} Y \mathrm{P})=-857.7460529$

Freq $=-576.08$

$\begin{array}{lccc}\mathrm{C} & -4.64183 & 0.80303 & 3.27476 \\ \mathrm{~N} & -3.77806 & 0.66853 & 2.10750 \\ \mathrm{H} & -4.87291 & 1.86412 & 3.40254 \\ \mathrm{H} & -4.06973 & 0.45735 & 4.13886 \\ \mathrm{H} & -5.61121 & 0.16439 & 3.16316 \\ \mathrm{C} & -3.85205 & 1.35684 & 0.93005 \\ \mathrm{C} & -4.79730 & 2.48450 & 0.68890 \\ \mathrm{H} & -5.83624 & 2.17246 & 0.83976 \\ \mathrm{H} & -4.68914 & 2.84636 & -0.33642 \\ \mathrm{H} & -4.59616 & 3.32013 & 1.37029 \\ \mathrm{C} & -2.88718 & 0.77268 & 0.13054 \\ \mathrm{~N} & -2.31232 & -0.20684 & 0.86432 \\ \mathrm{~N} & -2.84574 & -0.28511 & 2.06403 \\ \mathrm{H} & -2.57141 & 0.98656 & -0.87868 \\ \mathrm{C} & 0.42315 & 0.04640 & -0.80712 \\ \mathrm{O} & -0.32460 & 0.05965 & -1.76305 \\ \mathrm{C} & 1.85118 & 0.57565 & -0.77206 \\ \mathrm{C} & 2.21747 & 1.10862 & -2.16518 \\ \mathrm{C} & 2.77880 & -0.59973 & -0.37883 \\ \mathrm{C} & 1.94822 & 1.70542 & 0.27725 \\ \mathrm{H} & 2.17811 & 0.31161 & -2.91475 \\ \mathrm{H} & 3.23376 & 1.51711 & -2.14665 \\ \mathrm{H} & 1.52973 & 1.90111 & -2.47730 \\ \mathrm{H} & 1.30205 & 2.55047 & 0.01191 \\ \mathrm{H} & 2.98111 & 2.06859 & 0.32699 \\ \mathrm{H} & 1.66088 & 1.34707 & 1.27023 \\ \mathrm{H} & 2.52576 & -1.50175 & -0.94547 \\ \mathrm{H} & 2.66888 & -0.84066 & 0.68288 \\ \mathrm{H} & 3.82280 & -0.32439 & -0.56636 \\ \mathrm{O} & -6.91727 & -0.84494 & 3.24762 \\ \mathrm{H} & -6.35391 & -1.46759 & 3.74070 \\ \mathrm{O} & 0.33229 & -2.91078 & -0.23370 \\ \mathrm{H} & 1.04243 & -2.69205 & 0.38991 \\ \mathrm{C} & -1.00750 & -1.34740 & 0.39953 \\ \mathrm{H} & -1.43213 & -1.64630 & -0.54077 \\ \mathrm{H} & -1.08216 & -1.96527 & 1.27598 \\ \mathrm{O} & 0.05797 & -0.46163 & 0.40519 \\ & & & \\ & & & \end{array}$


5.4. Comparison of the free energy profiles of the intramolecular and intermolecular thermal cycloaddition reaction of 4-ethynyl-1-methyl-1,2,3-triazolium cations. Electronic energies and cartesian coordinates.

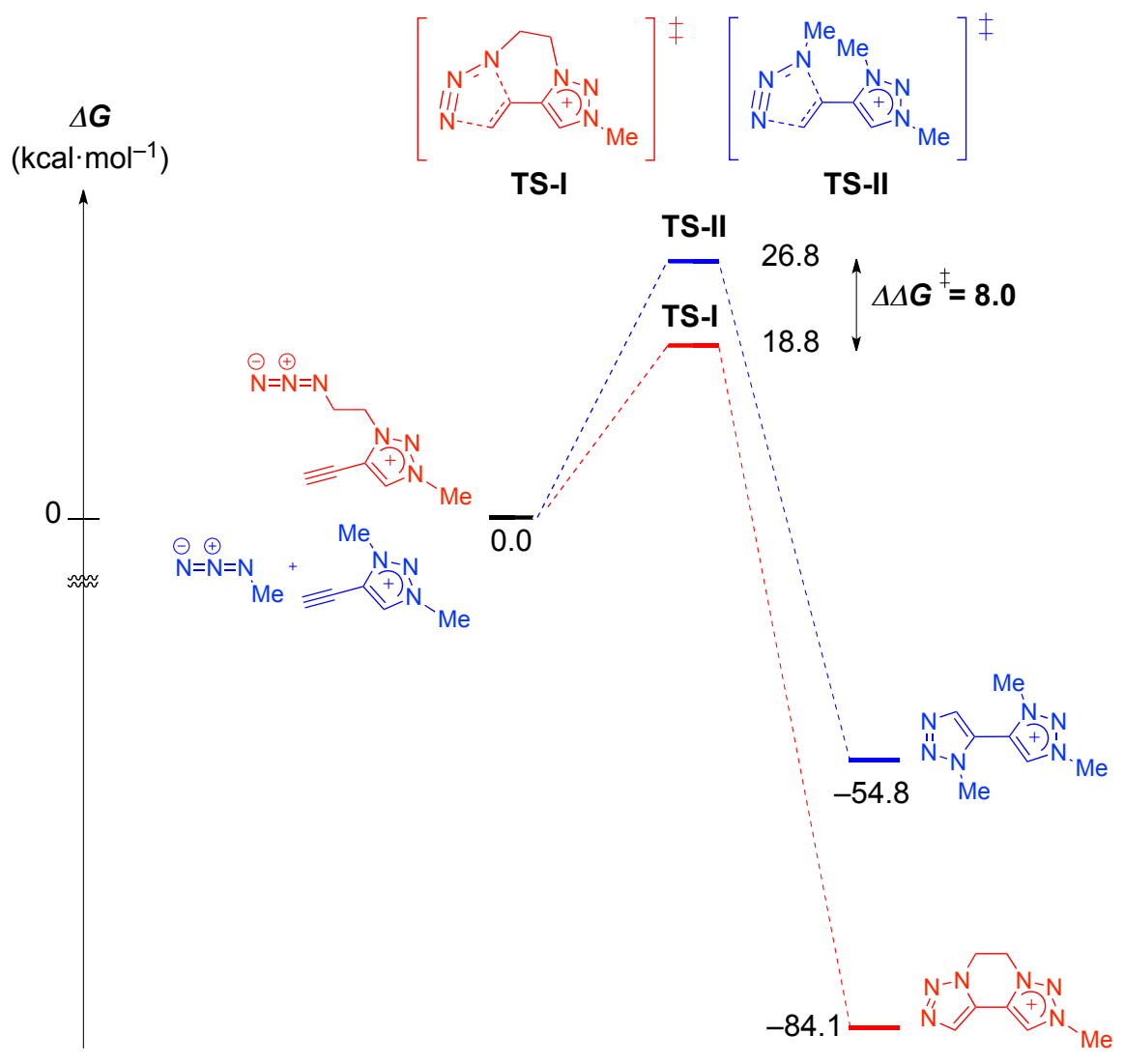

Figure S7. Transition states and reaction products computed at B3LYP/6-31G* level of theory for the intramolecular (red) and intermolecular (blue) thermal cycloaddition of the 4-ethynyl-1,2,3-triazolium cations. Relative $\Delta G^{\ddagger}$ energies in $\mathrm{kcal} \cdot \mathrm{mol}^{-1}$ calculated at $25^{\circ} \mathrm{C}$ in $\mathrm{MeCN}$. 


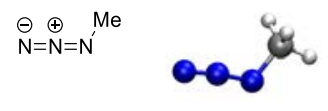

$E(R-B 3 L Y P)=-203.9845863611$

$\begin{array}{lrrr}\mathrm{C} & 1.64097 & -0.35702 & -0.00089 \\ \mathrm{~N} & 0.50317 & 0.59169 & -0.00663 \\ \mathrm{H} & 1.64406 & -0.97977 & -0.90353 \\ \mathrm{H} & 1.61789 & -1.00199 & 0.88557 \\ \mathrm{H} & 2.54804 & 0.24853 & 0.01967 \\ \mathrm{~N} & -0.61980 & 0.08847 & -0.00029 \\ \mathrm{~N} & -1.71211 & -0.25252 & 0.00631\end{array}$

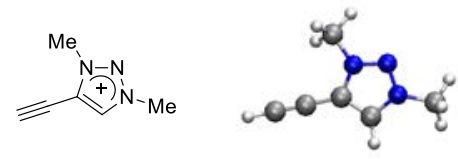

$E(R-B 3 L Y P)=-397.2264736894$

$\begin{array}{lrrr}\mathrm{C} & -3.17584 & -0.99026 & 0.00457 \\ \mathrm{H} & -4.22523 & -1.20919 & 0.02938 \\ \mathrm{C} & -1.99444 & -0.73767 & -0.01273 \\ \mathrm{C} & -0.62425 & -0.41274 & -0.01897 \\ \mathrm{C} & 0.51393 & -1.19947 & -0.02294 \\ \mathrm{~N} & 1.16180 & 0.92971 & 0.00787 \\ \mathrm{~N} & 1.55274 & -0.33178 & -0.00453 \\ \mathrm{C} & 2.98756 & -0.64690 & 0.02820 \\ \mathrm{~N} & -0.15239 & 0.87750 & -0.00293 \\ \mathrm{C} & -0.93909 & 2.11501 & -0.01171 \\ \mathrm{H} & -0.24098 & 2.95096 & -0.03517 \\ \mathrm{H} & -1.55361 & 2.15295 & 0.89052 \\ \mathrm{H} & -1.57269 & 2.12215 & -0.90127 \\ \mathrm{H} & 0.62910 & -2.27329 & -0.04239 \\ \mathrm{H} & 3.22332 & -1.30055 & -0.81404 \\ \mathrm{H} & 3.22498 & -1.13709 & 0.97488 \\ \mathrm{H} & 3.53569 & 0.29097 & -0.06165\end{array}$
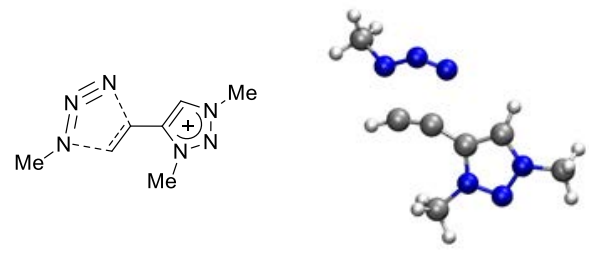

$\mathrm{E}(\mathrm{R}-\mathrm{B} 3 \mathrm{LYP})=-601.1924791323$

Freq $=-416.801$

$\begin{array}{lrrr}\mathrm{H} & -4.60577 & -0.12810 & 1.29728 \\ \mathrm{C} & -4.65870 & 0.18880 & 0.24959 \\ \mathrm{H} & -5.41485 & -0.40055 & -0.27966 \\ \mathrm{H} & -4.94351 & 1.24186 & 0.20082 \\ \mathrm{~N} & -3.35663 & 0.07537 & -0.42041 \\ \mathrm{~N} & -2.75494 & -1.02254 & -0.43751 \\ \mathrm{~N} & -1.69129 & -1.49485 & -0.44001 \\ \mathrm{C} & -1.68224 & 1.28379 & 0.03915 \\ \mathrm{H} & -2.23027 & 2.19995 & 0.15127 \\ \mathrm{C} & -0.70462 & 0.52147 & 0.00346 \\ \mathrm{C} & 0.66218 & 0.16341 & 0.03246 \\ \mathrm{C} & 1.30972 & -1.05164 & 0.17618 \\ \mathrm{H} & 0.91953 & -2.04734 & 0.32155 \\ \mathrm{~N} & 2.87379 & 0.51531 & -0.04972 \\ \mathrm{~N} & 2.63287 & -0.77178 & 0.12452 \\ \mathrm{C} & 3.75960 & -1.70941 & 0.20515 \\ \mathrm{H} & 4.66369 & -1.12652 & 0.38240 \\ \mathrm{H} & 3.84042 & -2.26013 & -0.73448 \\ \mathrm{H} & 3.58758 & -2.39407 & 1.03766 \\ \mathrm{~N} & 1.68432 & 1.07680 & -0.09542 \\ \mathrm{C} & 1.56034 & 2.52225 & -0.29251 \\ \mathrm{H} & 1.11141 & 2.71493 & -1.27007 \\ \mathrm{H} & 2.55920 & 2.95625 & -0.24114 \\ \mathrm{H} & 0.92876 & 2.93271 & 0.49893\end{array}$



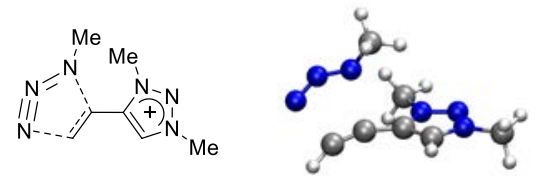

$\mathrm{E}(\mathrm{R}-\mathrm{B} 3 \mathrm{LYP})=-601.1893653460$

Freq $=-461.870$

$\begin{array}{lrrr}\mathrm{H} & 1.71658 & 1.68138 & -2.20919 \\ \mathrm{C} & 1.53204 & 1.97173 & -1.16881 \\ \mathrm{H} & 2.08417 & 2.88819 & -0.93308 \\ \mathrm{H} & 0.46680 & 2.17090 & -1.03288 \\ \mathrm{~N} & 1.88050 & 0.90054 & -0.22936 \\ \mathrm{~N} & 3.00740 & 0.35929 & -0.28297 \\ \mathrm{~N} & 3.51745 & -0.68234 & -0.10015 \\ \mathrm{C} & 0.83565 & -1.09090 & 0.03105 \\ \mathrm{C} & 1.81942 & -1.83898 & 0.13418 \\ \mathrm{C} & -0.49606 & -0.61743 & -0.00204 \\ \mathrm{C} & -1.50149 & -0.74028 & -0.94700 \\ \mathrm{~N} & -2.30801 & 0.46154 & 0.74683 \\ \mathrm{~N} & -2.56123 & -0.06863 & -0.43383 \\ \mathrm{C} & -3.90423 & 0.05791 & -1.01060 \\ \mathrm{H} & -4.42454 & 0.86140 & -0.48928 \\ \mathrm{H} & -4.43632 & -0.88740 & -0.87887 \\ \mathrm{H} & -3.81211 & 0.29386 & -2.07188 \\ \mathrm{H} & 2.29302 & -2.78914 & 0.29969 \\ \mathrm{~N} & -1.05593 & 0.13338 & 1.00092 \\ \mathrm{C} & -0.41122 & 0.56125 & 2.24419 \\ \mathrm{H} & -1.12879 & 1.14918 & 2.81492 \\ \mathrm{H} & 0.46495 & 1.16183 & 1.99109 \\ \mathrm{H} & -0.10654 & -0.32328 & 2.80875 \\ \mathrm{H} & -1.51282 & -1.24116 & -1.90421\end{array}$

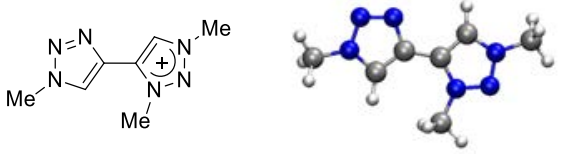

$E(R-B 3 L Y P)=-601.3386433$

$\begin{array}{lrrr}\mathrm{H} & -4.60876 & 1.01521 & 1.07884 \\ \mathrm{C} & -4.36586 & 0.73081 & 0.05083 \\ \mathrm{H} & -5.06424 & -0.03189 & -0.29728 \\ \mathrm{H} & -4.42171 & 1.60611 & -0.59880 \\ \mathrm{~N} & -3.01861 & 0.16841 & -0.00127 \\ \mathrm{~N} & -2.83479 & -1.16861 & 0.06094 \\ \mathrm{~N} & -1.55291 & -1.39298 & 0.07054 \\ \mathrm{C} & -1.83729 & 0.81929 & -0.02883 \\ \mathrm{H} & -1.77759 & 1.89516 & -0.07895 \\ \mathrm{C} & -0.89198 & -0.19436 & 0.01832 \\ \mathrm{C} & 0.55456 & -0.19785 & 0.02781 \\ \mathrm{C} & 1.40580 & -1.28798 & 0.07031 \\ \mathrm{H} & 1.19584 & -2.34520 & 0.11279 \\ \mathrm{~N} & 2.66537 & 0.54261 & 0.00826 \\ \mathrm{~N} & 2.65712 & -0.77347 & 0.05385 \\ \mathrm{C} & 3.92787 & -1.50809 & 0.04249 \\ \mathrm{H} & 4.73291 & -0.80026 & 0.23903 \\ \mathrm{H} & 4.06087 & -1.97393 & -0.93678 \\ \mathrm{H} & 3.89731 & -2.26845 & 0.82521 \\ \mathrm{~N} & 1.38694 & 0.88623 & -0.00827 \\ \mathrm{C} & 1.03859 & 2.30730 & -0.05430 \\ \mathrm{H} & 0.47103 & 2.51233 & -0.96552 \\ \mathrm{H} & 1.96792 & 2.87718 & -0.06619 \\ \mathrm{H} & 0.46222 & 2.56838 & 0.83648\end{array}$



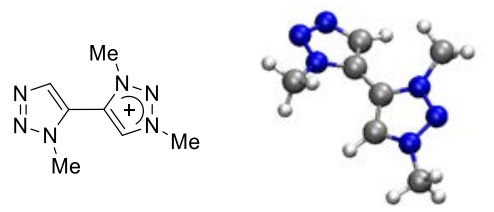

E $(\mathrm{R}-\mathrm{B} 3 \mathrm{~L}$ YP $)=-601.3308627$

$\begin{array}{lrrr}\mathrm{H} & 1.16576 & 1.04372 & -2.77363 \\ \mathrm{C} & 1.50997 & 1.55133 & -1.86832 \\ \mathrm{H} & 2.39578 & 2.14194 & -2.10493 \\ \mathrm{H} & 0.73005 & 2.21015 & -1.47945 \\ \mathrm{~N} & 1.89038 & 0.57955 & -0.84674 \\ \mathrm{~N} & 3.18556 & 0.30729 & -0.63478 \\ \mathrm{~N} & 3.26610 & -0.57245 & 0.33578 \\ \mathrm{C} & 1.10834 & -0.14310 & 0.00973 \\ \mathrm{C} & 2.01593 & -0.87443 & 0.76154 \\ \mathrm{C} & -0.33851 & -0.07663 & 0.02908 \\ \mathrm{C} & -1.26435 & 0.00778 & -0.99594 \\ \mathrm{~N} & -2.38446 & 0.00686 & 0.92155 \\ \mathrm{~N} & -2.47394 & 0.06226 & -0.39156 \\ \mathrm{C} & -3.80099 & 0.11423 & -1.01813 \\ \mathrm{H} & -4.39127 & 0.89025 & -0.52875 \\ \mathrm{H} & -4.28214 & -0.85964 & -0.90225 \\ \mathrm{H} & -3.67719 & 0.34523 & -2.07573 \\ \mathrm{H} & 1.83970 & -1.58370 & 1.55789 \\ \mathrm{~N} & -1.09387 & -0.07283 & 1.17249 \\ \mathrm{C} & -0.64960 & -0.16425 & 2.56869 \\ \mathrm{H} & -1.45819 & 0.20645 & 3.19842 \\ \mathrm{H} & 0.24100 & 0.45395 & 2.69729 \\ \mathrm{H} & -0.43704 & -1.20773 & 2.81241 \\ \mathrm{H} & -1.13834 & 0.00659 & -2.06719\end{array}$

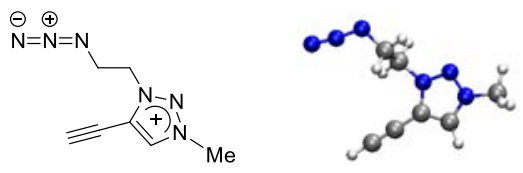

$\mathrm{E}(\mathrm{R}-\mathrm{B} 3 \mathrm{LYP})=-600.0182791$

$\begin{array}{lrrr}\mathrm{C} & -0.56372 & -0.19155 & 0.96650 \\ \mathrm{H} & -0.88961 & -1.20840 & 1.19548 \\ \mathrm{H} & -1.39566 & 0.50534 & 1.06606 \\ \mathrm{H} & 0.26043 & 0.10371 & 1.61911 \\ \mathrm{~N} & -0.10572 & -0.14200 & -0.42858 \\ \mathrm{C} & 0.87078 & -0.87627 & -1.01146 \\ \mathrm{H} & 1.44709 & -1.63615 & -0.50541 \\ \mathrm{C} & 0.91612 & -0.45145 & -2.32755 \\ \mathrm{~N} & -0.68194 & 0.69760 & -1.26721 \\ \mathrm{~N} & -0.05590 & 0.51800 & -2.41221 \\ \mathrm{C} & -0.48296 & 1.26080 & -3.60768 \\ \mathrm{H} & 0.39299 & 1.35840 & -4.25236 \\ \mathrm{H} & -0.80293 & 2.25243 & -3.28308 \\ \mathrm{C} & -1.62232 & 0.50948 & -4.32347 \\ \mathrm{H} & -1.30260 & -0.49649 & -4.61760 \\ \mathrm{H} & -2.48013 & 0.42199 & -3.65162 \\ \mathrm{~N} & -2.06442 & 1.30903 & -5.48218 \\ \mathrm{~N} & -1.60106 & 0.97381 & -6.57759 \\ \mathrm{~N} & -1.26099 & 0.75845 & -7.64486 \\ \mathrm{C} & 1.71953 & -0.85037 & -3.41334 \\ \mathrm{H} & 3.00464 & -1.47217 & -5.19195 \\ \mathrm{C} & 2.39836 & -1.18318 & -4.35600\end{array}$



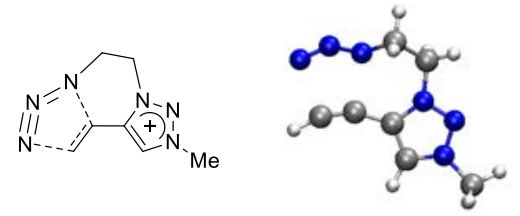

$\mathrm{E}(\mathrm{R}-\mathrm{B} 3 \mathrm{~L} Y \mathrm{P})=-599.9890362$

Freq $=-457.459$

$\begin{array}{lrrr}\mathrm{C} & -4.10915 & -0.49206 & -0.15640 \\ \mathrm{H} & -4.44734 & -1.13832 & 0.65630 \\ \mathrm{H} & -4.33125 & -0.94714 & -1.12426 \\ \mathrm{H} & -4.57761 & 0.48858 & -0.07968 \\ \mathrm{~N} & -2.65654 & -0.30836 & -0.03665 \\ \mathrm{C} & -1.70341 & -1.26380 & 0.03700 \\ \mathrm{H} & -1.91848 & -2.32235 & 0.02746 \\ \mathrm{C} & -0.49653 & -0.58923 & 0.13255 \\ \mathrm{~N} & -2.15599 & 0.90952 & 0.00159 \\ \mathrm{~N} & -0.85104 & 0.74331 & 0.09571 \\ \mathrm{C} & -0.02067 & 1.96238 & 0.22870 \\ \mathrm{H} & 0.10432 & 2.15372 & 1.29866 \\ \mathrm{H} & -0.59020 & 2.77778 & -0.21870 \\ \mathrm{C} & 1.34171 & 1.80041 & -0.43535 \\ \mathrm{H} & 1.25083 & 1.60135 & -1.51035 \\ \mathrm{H} & 1.90537 & 2.72883 & -0.29037 \\ \mathrm{~N} & 3.04792 & 0.17277 & -0.09807 \\ \mathrm{C} & 0.81957 & -1.12545 & 0.15467 \\ \mathrm{C} & 1.66784 & -2.02248 & 0.04927 \\ \mathrm{H} & 2.10759 & -2.99752 & -0.01903 \\ \mathrm{~N} & 3.56613 & -0.86383 & -0.22093 \\ \mathrm{~N} & 1.96690 & 0.68516 & 0.27820\end{array}$
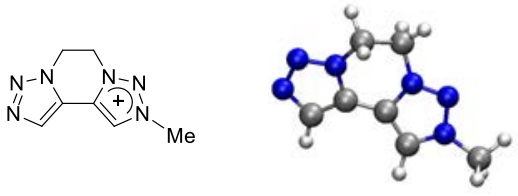

$E(R-B 3 L Y P)=-600.1314910$

$\begin{array}{llll}\text { C } & 0.06901 & -0.26779 & 0.93760\end{array}$

$\begin{array}{llll}\mathrm{H} & -0.50259 & -1.15277 & 1.22726\end{array}$

$\begin{array}{llll}\mathrm{H} & -0.31463 & 0.61796 & 1.44308\end{array}$

$\begin{array}{llll}\mathrm{H} & 1.12740 & -0.39980 & 1.17004\end{array}$

$\begin{array}{llll}\mathrm{N} & -0.07412 & -0.05528 & -0.50803\end{array}$

$\begin{array}{llll}\text { C } & 0.38219 & -0.84925 & -1.51159\end{array}$

$\begin{array}{llll}\mathrm{H} & 0.93601 & -1.76280 & -1.34736\end{array}$

$\begin{array}{llll}\mathrm{C} & -0.04443 & -0.24165 & -2.67384\end{array}$

$\begin{array}{llll}\mathrm{N} & -0.75363 & 0.98870 & -0.94460\end{array}$

$\begin{array}{llll}\mathrm{N} & -0.72834 & 0.86777 & -2.25383\end{array}$

$\begin{array}{llll}\mathrm{C} & -1.32140 & 1.86156 & -3.17190\end{array}$

$\begin{array}{llll}\mathrm{H} & -0.53234 & 2.57028 & -3.44298\end{array}$

$\begin{array}{llll}\mathrm{H} & -2.12199 & 2.38548 & -2.64781\end{array}$

$\begin{array}{llll}\text { C } & -1.85219 & 1.10949 & -4.39828\end{array}$

$\begin{array}{llll}\mathrm{H} & -2.72409 & 0.49998 & -4.13987\end{array}$

$\begin{array}{llll}\mathrm{H} & -2.13434 & 1.81455 & -5.18162\end{array}$

$\begin{array}{llll}\mathrm{N} & -0.62518 & -0.11335 & -6.18229\end{array}$

$\begin{array}{llll}\text { C } & 0.03550 & -0.49206 & -4.09068\end{array}$

$\begin{array}{llll}\text { C } & 0.74724 & -1.29739 & -4.96052\end{array}$

$\begin{array}{llll}\mathrm{H} & 1.53457 & -2.00836 & -4.75347\end{array}$

$\begin{array}{llll}\mathrm{N} & 0.31617 & -1.03155 & -6.22227\end{array}$

$\begin{array}{llll}\mathrm{N} & -0.79182 & 0.23030 & -4.90005\end{array}$ 